Portland State University

PDXScholar

Fall 12-5-2017

\title{
Copper Nanowires Synthesis and Self-Assembly for Interconnect Applications
}

Srikar Rao Darmakkolla

Portland State University

Follow this and additional works at: https://pdxscholar.library.pdx.edu/open_access_etds

Part of the Chemistry Commons

Let us know how access to this document benefits you.

\section{Recommended Citation}

Darmakkolla, Srikar Rao, "Copper Nanowires Synthesis and Self-Assembly for Interconnect Applications" (2017). Dissertations and Theses. Paper 4034.

https://doi.org/10.15760/etd.5918

This Dissertation is brought to you for free and open access. It has been accepted for inclusion in Dissertations and Theses by an authorized administrator of PDXScholar. Please contact us if we can make this document more accessible: pdxscholar@pdx.edu. 
Copper Nanowires Synthesis and Self-Assembly for Interconnect Applications

by

Srikar Rao Darmakkolla

A dissertation submitted in partial fulfillment of the requirements for the degree of

Doctor of Philosophy

in

Chemistry

Dissertation Committee:

Shankar B. Rananavare, Chair

Robert Strongin

Dean Atkinson

Gwendolyn Shusterman

Andres La Rosa

Charlie Montross

Portland State University

2017 
@ 2017 Srikar Rao Darmakkolla 


\begin{abstract}
One-dimensional (1D) nanomaterial self-assembly offers an excellent approach to the fabrication of highly complex nanodevices. Despite considerable effort and research, precisely controlling the orientation and positioning of nanowires (NWs) on a large-scale area and assembling into a functional device is still a state of the art problem. This thesis focuses on the dimensionally controlled copper nanowires $(\mathrm{Cu} \mathrm{NWs})$ synthesis, and magnetic field assisted self-assembly of cupronickel nanowires ( $\mathrm{Cu} / \mathrm{Ni} \mathrm{NWs}$ ) into interconnect structures on a carbon doped silicon dioxide (CDO) wafer.

$\mathrm{CDO}$ is a low dielectric constant $(k)$ material used for copper interconnects in multilayered complex integrated circuits (ICs). Here, a strong affinity of copper $(\mathrm{Cu})$ and nickel (Ni) to thiol (-SH) functional groups were exploited to strongly adhere the nanowires $(\mathrm{Cu} / \mathrm{Ni} \mathrm{NWs})$ onto the CDO substrate. Thiol (-SH) functionalization of the CDO surface was achieved via a series of reactions involving (1) esterification of the surface exposed $\equiv \mathrm{Si}-\mathrm{OH}$ functional group to its triflate $(\equiv \mathrm{Si}-\mathrm{O}-\mathrm{Tf}),(2)$ reduction of triflate to $\equiv \mathrm{Si}-\mathrm{H}$ using DIBAL-H, and (3) hydrosilylation of $\equiv \mathrm{Si}-\mathrm{H}$ using 2-propene thiol $\left(\equiv \mathrm{Si}-\left(\mathrm{CH}_{2}\right)_{3}-\mathrm{SH}\right)$ in a photochemical reaction. The thiol functionalization of CDO surface enhances the interaction of $\mathrm{Cu} / \mathrm{Ni} \mathrm{NWs}$ with strong chemical bonds. The same reaction scheme was also used in the functionalization of the hydrophilic $(\mathrm{Si}-\mathrm{OH})$ surface to the hydrophobic long alkyl chain derivatized $\left(\equiv \mathrm{Si}-\mathrm{CH}_{2}-\left(\mathrm{CH}_{2}\right)_{16}-\mathrm{CH}_{3}\right)$ surface. This long alkyl chain modified surface acts as an excellent moisture resistant film, which helps to maintain the low- $k$ value of the CDO.
\end{abstract}


The dimensionally controlled $\mathrm{Cu}$ NWs were synthesized by a wet chemical approach. Optimization of the reducing agent, hydrazine $\left(\mathrm{N}_{2} \mathrm{H}_{4}\right)$, controlled the surface morphology of nanowires (NWs). Interestingly, the high concentration of reducing agent produced particle decorated and/or with a rough NW surface, and conversely decreasing its concentration resulted in a comparatively thin, particle-free and smooth surface. The reaction temperature affected the aspect ratio (Length/Diameter) of the NWs. As the reaction temperature increased from 60 to $90{ }^{\circ} \mathrm{C}$, the aspect ratio decreased from 140 to 21.

Controlling the orientation of $\mathrm{Cu}$ NWs in a magnetic field was accomplished by coating them with a thin layer $(20 \mathrm{~nm})$ of ferromagnetic nickel (Ni). This Ni-coated NWs showed an excellent degree of alignment (half-width $\approx 10$ degrees) in the direction of an applied magnetic field over a large surface area at field strength as low as 2500 Gauss. Also, the Ni coating helped in protecting the copper core from oxidation resulting in better electrical wire-to-wire contacts.

A nanowire-based interconnect channel was fabricated by combining magnetic field assisted alignment and deposition of aligned NWs on a thiol-modified and photolithography patterned CDO substrate. The NWs, deposited in the trenches, strongly bonded to the thiol-derivatized CDO substrate while an acetone wash removed loosely bound NWs on the photoresist surface. In electrical characterization, the directionally wellaligned $\mathrm{Cu} / \mathrm{Ni}$ NWs channel displayed surprisingly two-fold higher conductivity than randomly arranged NWs channel. 


\section{DEDICATION}

This dissertation is dedicated to my parents and all my family members. 


\section{ACKNOWLEDGEMENTS}

I would like to express my deepest gratitude towards my research advisor Dr. Shankar B. Rananavare for his motivation, support, guidance, and encouragement during my studies at Portland State University. I am thankful for teaching me how to approach the difficult problems in research and allowing me to grow as a research scientist. I am blessed to have you as my supervisor for my Ph.D. study.

I am indebted to my dissertation committee members: Dr. Robert Strongin, Dr. Dean Atkinson, Dr. Gwen Shusterman, Dr. Andres La Rosa and Dr. Charlie Monstross for their contributions towards completion of my degree requirements. I am also grateful to the faculty and staff members of Chemistry Department, PSU for their support, including uninterrupted financial support during my studies.

I would also want to thank past and current members of the Rananavare's research group; Dr. Nabil Mistkawi, Dr. Kayode Morakinyo, Dr. Ruchi Gakhar, Dr. Hoang Tran, Atul Gupta, Mitra Ghobadi, Ryan Lerud, Drew Beseau, Fardowsa Idris, Patricia Monsilla, Ana Jenike, Amanda F.Pereira, Irene R. Aguilar, Evangeline Liu and Emmanuel Abdul for their suggestions and invaluable support. I am also grateful to the all high school interns; Musa Tahir, Nikhil Murthy, Lauren Cooper and Vineet Edupuganti for their help in carrying out some of my experiments and I have learned valuable supervising experience after closely guiding them in the lab.

I want to express my sincere gratitude to Dr. Tahir Ghani, Intel for his invaluable supervision for my thesis and career. I am also grateful to Dr. Deepak Rajput, Intel for his 
mentorship and career guidance. Also, special thanks are due to Dr. Raj Solanki, Dr. Andres La Rosa and their group members for their kind access and help in SEM, AFM, and optical microscopy characterization. Finally, I also thank Tony Chen and Greg Baty, CEMN, for their help in SEM, TEM, t-EBSD characterization work. I thank my friend Susan Caisse for her help in editing my manuscripts and valuable friendship.

I greatly appreciate the love and support of my wife Haindhavi for being there for me, in every way and her words of encouragement. I am indebted to my parents for their endless support, love, and encouragement over the years and it was the greatest gift anyone has ever given me. My profound gratitude goes to my brother Satish Rao, for his moral support and encouragement. Finally, I give glory to the God that I believe in for giving me faith to trust in him. 


\section{TABLE OF CONTENTS}

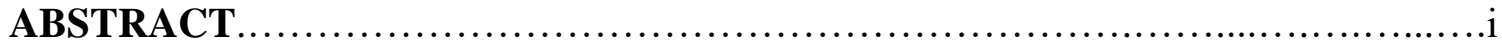

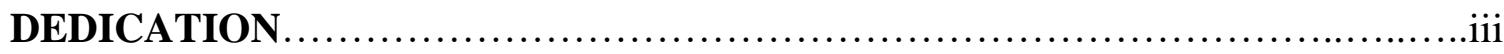

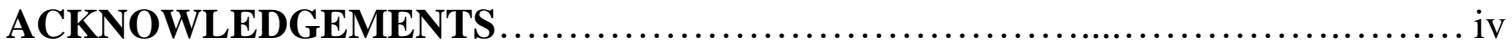

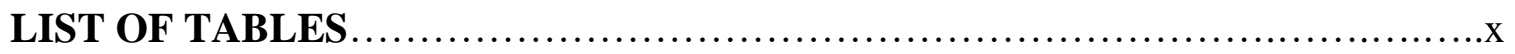

LIST OF FIGURES ...................................................................

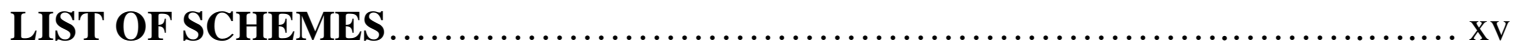

LIST OF ABBREVIATIONS ................................................

CHAPTER ONE: INTRODUCTION AND RATIONALE .........................

1.1. Review on traditional process of copper interconnects fabrication on chips.........2

1.2. Challenges in modern integrated circuit fabrication........................... 6

1.3. Alternate materials and methods for interconnect fabrication.................... 7

1.4. Thesis objectives and outlines........................................... 16

CHAPTER TWO: INSTRUMENTATION, MATERIALS AND METHODS........21

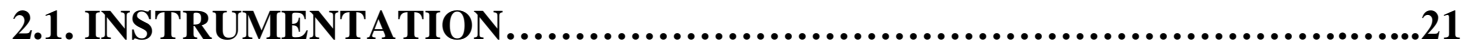

2.1.1. Fourier transform infrared spectroscopy (FTIR)..........................21

2.1.2. Nuclear magnetic resonance (NMR) spectroscopy..........................21

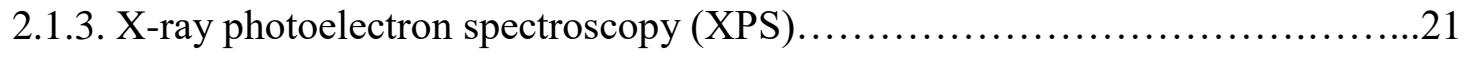

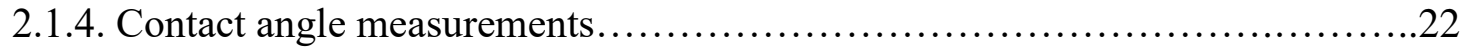

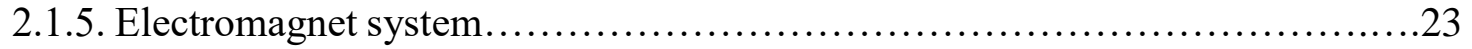

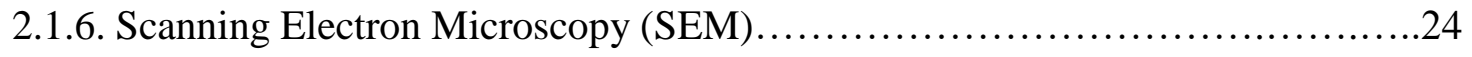

2.1.7. Transmission Electron Microscopy (TEM)...................................26

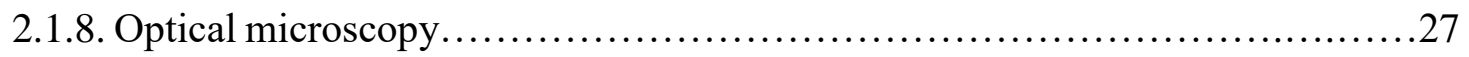

2.2. MATERIALS AND METHODS.........................................27

2.2.1. Materials used for $\mathrm{Cu}$ NWs synthesis, nickel electroless plating, surface functionalization of CDO wafer and photolithography..............................27 


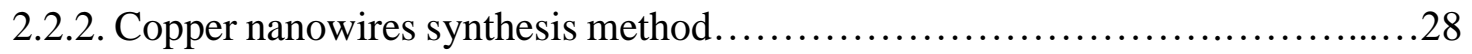

2.2.3. Electroless coating of nickel on $\mathrm{Cu}$ NWs surface..........................28

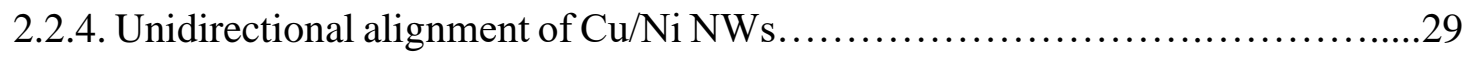

2.2.5. Common synthetic procedure for selective functionalization of $\mathrm{Si}-\mathrm{OH}$

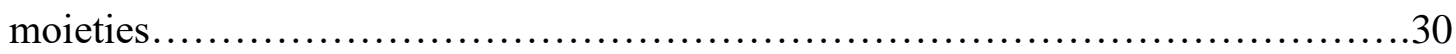

2.2.6. RCA cleaning procedure for $\mathrm{SiOx}$ and $\mathrm{CDO}$ wafers.........................

2.2.7. Photolithography.................................................. 32

2.2.8. Electrical characterization of self-assembled interconnect channels.............34

CHAPTER THREE: COPPER NANOWIRES SYNTHESIS AND MAGNETIC

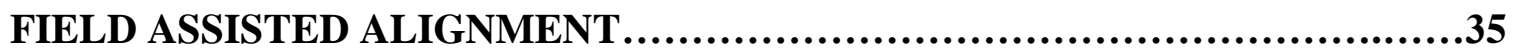

3.1. Copper nanowires synthesis...........................................

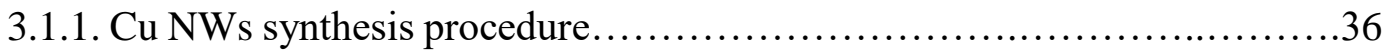

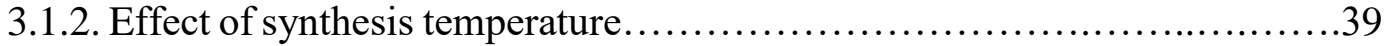

3.1.3. Effect of reducing agent concentration...............................41

3.2. Electroless plating of nickel (Ni) on $\mathrm{Cu}$ NWs surface.........................47

3.2.1. Nickel electroless plating procedure $\ldots \ldots \ldots \ldots \ldots \ldots \ldots \ldots \ldots \ldots \ldots \ldots . \ldots \ldots$

3.3. Magnetic field assisted alignment.....................................49

3.3.1. Effect of magnetic field strength on NWs alignment order.................52

3.4. Summary of copper nanowires synthesis and magnetic field assisted

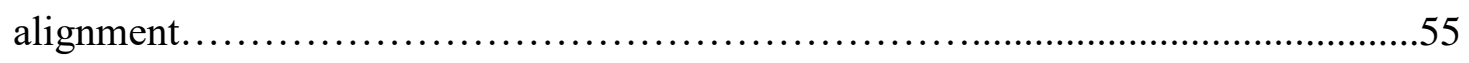

\section{CHAPTER FOUR: SURFACE DERIVATIZATION ON CARBON DOPED} SILICON OXIDE (CDO) WAFER............................................57

4.1. Introduction...................................................... 57

4.2. CDO as a low- $k$ dielectric material.....................................60

4.3. Review on CDO film preparation and chemical structure....................61

4.4. Review on CDO damage and repairing methodologies......................62

4.4.1. Review on CDO damage by plasma processing......................62 
4.4.2. Review on CDO repairing methodologies..............................64

4.5. Results of developed pathway for selective derivatization of silanol...............65

4.5.1. Developed scheme for surface derivatization............................65

4.5.2. Results on organic molecules.......................................66

a) tert-Butyldimethylsilanol............................................

b) tert-Butyltrimethylsilane........................................ 71

c) Ethoxytriphenylsilane........................................... 72

4.5.3. Results on silica gel reaction.......................................73

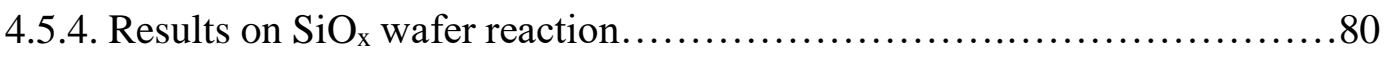

4.5.5. Results on CDO wafer reaction..................................... 83

4.5.6. Comparison of octadecyl chain derivatized via DIBAL-H chemistry and octadcyltrichlorosilane (OTS) routes...................................92

4.5.7. Characterization of film dielectric constants $(k) \ldots \ldots \ldots \ldots \ldots \ldots \ldots \ldots \ldots . . . . \ldots 3$

4.6. Thiol functionalization of CDO surface to enhance NWs interaction to

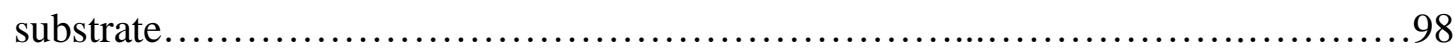

4.7. Summary of octadecyl and thiol functionalization.............................101

\section{CHAPTER FIVE: NANOWIRES SELF-ASSEMBLY AND ELECTRICAL}

CHARACTERIZATION.......................................................102

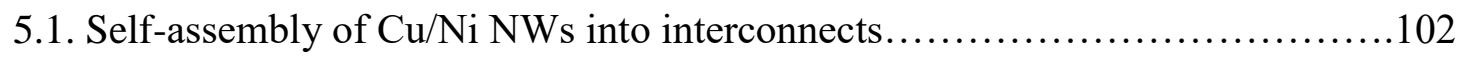

5.2. Electrical characterization of $\mathrm{Cu} / \mathrm{Ni} \mathrm{NWs}$ based interconnects..................104

5.2.1. Effect of nanowire type (pure $\mathrm{Cu}$ NWs vs. $\mathrm{Cu} / \mathrm{Ni} \mathrm{NWs}$ ) and arrangement (random vs. aligned) style on electrical performance.......................... 104

5.2.2. Effect of nanowires alignment order on electrical performance............108

5.2.3. Effect of NWs density on resistance of well-aligned $\mathrm{Cu} / \mathrm{Ni} \mathrm{NWs}$

channels................................................................... 110

5.2.4. Thermal stability of $\mathrm{Cu} \mathrm{NWs}$ and $\mathrm{Cu} / \mathrm{Ni} \mathrm{NWs}$ based interconnect channels.................................................................. 111

5.3. Summary of $\mathrm{Cu} / \mathrm{Ni} \mathrm{NWs}$ self-assembly and electrical characterization...........113 


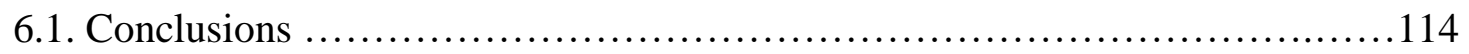

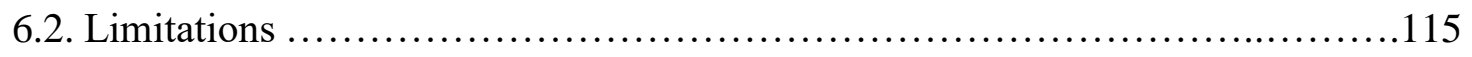

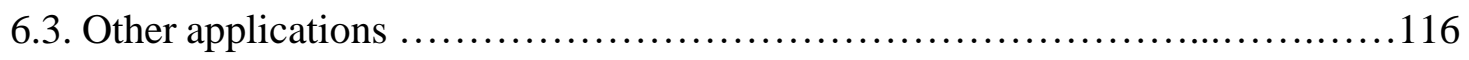

6.4. Future work ..................................................... 117

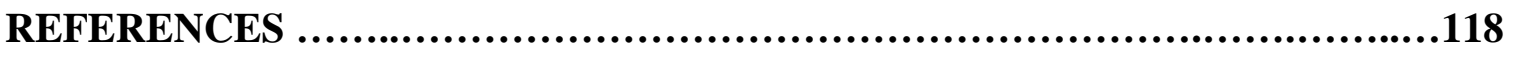

APPENDICES.............................................................132

Appendix-A. Protocol for nanowires length distribution measurement ................132

Appendix-B. Procedure for nanowires alignment angle measurement .................133

Appendix-C. Challenges in using ultra-long nanowires for the alignment...............134 


\section{LIST OF TABLES}

Table 2.1. Photolithography process steps and conditions

Table 3.1. Average lengths, diameters and aspect ratios of copper nanowires synthesized

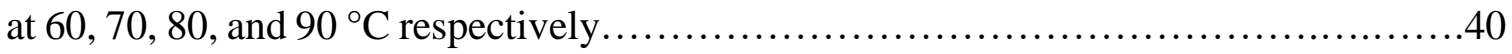

Table 3.2. List of field dependent parameters $\ldots \ldots \ldots \ldots \ldots \ldots \ldots \ldots \ldots \ldots \ldots \ldots \ldots \ldots \ldots 5$

Table 4.1. Dielectric constant $(k)$ values of various substances.....................60

Table 4.2. Kinetics of contact angle results of water droplet on clean CDO and octadecyl

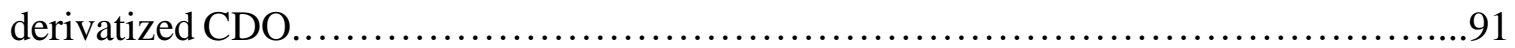

Table 4.3. Contact angle, film thicknesses of $\mathrm{CDO}$ and $\mathrm{SiO}_{\mathrm{x}}$ wafers..............96 


\section{LIST OF FIGURES}

Figure 1.1. Cross section SEM image of copper multilevel interconnect system with $\mathrm{CDO}$ as a low-k dielectric..........................................................

Figure 1.2. Schematic of DD process for making metal lines and vias..................

Figure 1.3. Schematic diagram of electroplating of copper on wafer....................5

Figure 1.4. Schematic showing various kinds of nanowires self-assembly .................9

Figure 1.5. Schematic diagram showing shear force induced self-assembly of NWs......13

Figure 1.6. Schematic illustrating electric field assisted alignment of NWs...............14

Figure 1.7. Schematic diagram illustrating the process of magnetic field assisted assembly of magnetically active nanowires.....................................................

Figure 1.8. Schematic diagram illustrating the $\mathrm{Cu} / \mathrm{Ni}$ NWs self-assembly...............16

Figure 1.9. Schematic diagram showing process flow of $\mathrm{Cu} / \mathrm{Ni}$ NWs self-assembly into

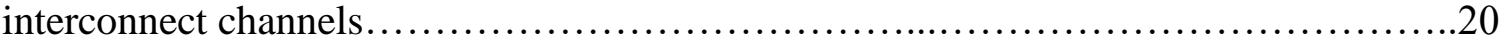

Figure 2.1. Water contact angle measurement setup.................................23

Figure 2.2. Plot showing change in magnetic field strength as a function of applied

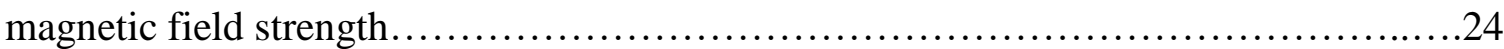

Figure 2.3. Schematic diagrams illustrating the difference between EBSD and t-EBSD processes of crystallographic orientation mapping in SEM............................25

Figure 2.4. Digital photographs showing custom-made sample holder for TKD data

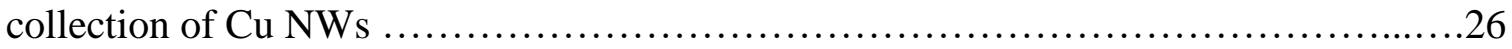

Figure 2.5. Digital photographs of GMW electromagnet setup ........................30

Figure 2.6. Schematic diagram showing photolithography process flow..................32

Figure 2.7. Digital photograph showing projection mask UV aligner.....................34

Figure 3.1. Digital images of $\mathrm{Cu}$ NWs reaction solution before adding hydrazine (a), after adding hydrazine (b), and after incubation for 30 minutes in hot water bath.

Figure 3.2. SEM images of copper nanowires synthesized at $60{ }^{\circ} \mathrm{C}(\mathrm{a}), 70{ }^{\circ} \mathrm{C}(\mathrm{b}), 80{ }^{\circ} \mathrm{C}$ (c), and $90{ }^{\circ} \mathrm{C}$ (d) respectively...................................................... 
Figure 3.3. SEM images of $\mathrm{Cu}$ NWs synthesized using high concentration $(35 \mu \mathrm{L})$ of reducing agent.

Figure 3.4. SEM images of copper nanowires synthesized using an intermediate concentration of hydrazine $\left(8.28 \times 10^{-3} \mathrm{M}, 30 \mu \mathrm{L}\right)$.

Figure 3.5. SEM images of $\mathrm{Cu}$ NWs synthesized using low concentration of reducing agent shown at low a) and high b) magnification scales .44

Figure 3.6. TEM image of $\mathrm{Cu} N W$ synthesized using $5.73 \times 10^{-3} \mathrm{M}(21 \mu \mathrm{L})$ concentration of hydrazine highlighting the particle-free surface.

Figure 3.7. Low magnification bright field a), and high magnification dark field b), TEM image showing the presence of nanoparticles

Figure 3.8. Digital photographs showing nickel electroless plating setup and magnetic response of $\mathrm{Cu} / \mathrm{Ni} \mathrm{NW}$ s towards neodymium disc magnets .48

Figure 3.9. SEM image of nickel coated copper nanowire a) and elemental mapping showing distribution of copper core and nickel coating on the surface.

Figure 3.10. Digital photographs of electromagnet setup.

Figure 3.11. Optical microscope images of $\mathrm{Cu} / \mathrm{Ni} \mathrm{NWs}$ orientation in absence (a), and in the presence of applied magnetic field (2500 Gauss) (b).

Figure 3.12. Optical micrographs showing alignment order of $\mathrm{Cu} / \mathrm{Ni} \mathrm{NWs}$ at various applied magnetic field strength from $0 \mathrm{G}$ to $2500 \mathrm{G}$.

Figure 3.13. Plot showing average NWs alignment angle with respect to the applied magnetic field direction, at various field strength from $0 \mathrm{G}$ to $2500 \mathrm{G}$ .54

Figure 3.14. Gaussian peak fitting plots showing NWs alignment distribution with respect to the applied magnetic field strength from 0 to 2500 Gauss

Figure 4.1. Image of multilayered integrated circuit (IC) using $\mathrm{Cu} / \mathrm{W} / \mathrm{SiO}_{2}$ dielectric

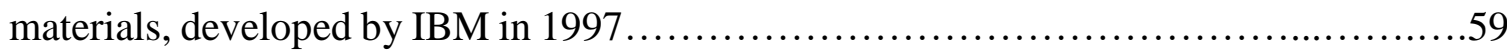

Figure 4.2. a) Chemical structure of porous cage networked structure of as-prepared CDO film from TMCTS precursor..............................................63

Figure 4.3. ${ }^{1} \mathrm{H}-\mathrm{NMR}\left(\mathrm{C}_{6} \mathrm{D}_{6}, 400 \mathrm{MHz}\right)$ reaction progress study of TBDMS Triflate synthesis at 2, 24, 48 and 72 hours respectively....

Figure 4.4. ${ }^{1} \mathrm{H}-\mathrm{NMR} \quad\left(\mathrm{CDCl}_{3}, 400 \mathrm{MHz}\right)$ reaction progress study of tertButyldimethylsilane synthesis at 2, 24, 48 and 72 hours respectively................. 70 
Figure 4.5. ${ }^{19} \mathrm{~F}-\mathrm{NMR} \quad\left(\mathrm{CDCl}_{3}, 400 \mathrm{MHz}\right)$ reaction progress study of tertButyldimethylsilane synthesis at 2, 24 and 48 hours respectively.....................71

Figure 4.6. ${ }^{1} \mathrm{H}-\mathrm{NMR}$ (DMSO- $\mathrm{d}_{6}$ ) reaction progress study of tert-Butyltrimethylsilane synthesis at 2,24 hours of reaction............................................. 72

Figure 4.7. ${ }^{1} \mathrm{H}-\mathrm{NMR}\left(\mathrm{DMSO}-\mathrm{D}_{6}\right)$ of triphenylsilane synthesis reaction................73

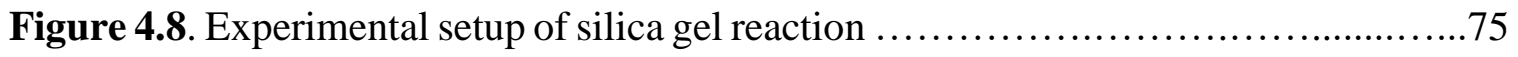

Figure 4.9. FTIR spectra of silica gel in various stages of reaction....................76

Figure 4.10. ${ }^{19}$ F NMR study of silica gel reaction with triflic anhydride..................78

Figure 4.11. FTIR of silica gel control experiment. Comparison of pure silica gel before reaction and after treatment with 1 -octadecene in the presence of UV light................80

Figure 4.12. FT-IR spectra of $\mathrm{SiO}_{x}$ wafer at various stages of the reaction........81

Figure 4.13. Common reaction pathway for hydrogen termination, octadecane derivatization and experimental setup of carbon doped silicon oxide (CDO) wafer........83

Figure 4.14. FT-IR spectra of CDO wafer in various stages of reaction.................84

Figure 4.15. Control experiment showing that only the porous structure of CDO traps DIBAL-H from anhydrous toluene solution.................................. 87

Figure 4.16. FTIR of CDO wafer control experiment. Spectral comparison of clean CDO before reaction and $\mathrm{CDO}$ after treatment with 1-octadecene.

Figure 4.17. XPS spectra of CDO wafer. Survey spectrum and high-resolution spectrums

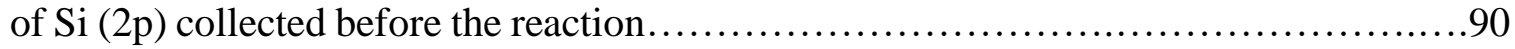

Figure 4.18. Water contact angles measured on un-derivatized (clean CDO) and octadecene derivatized CDO wafers at various time intervals........................91

Figure 4.19. FTIR spectra comparison of octadecyl derivatized CDO wafer before and

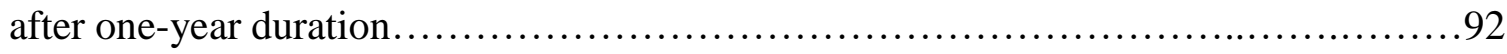

Figure 4.20. Comparison $\mathrm{CDO}$ and $\mathrm{SiO}_{\mathrm{x}}$ wafer derivatized by DIBAL-H assisted chemistry (blue) vs. OTS chemistry (red)

Figure 4.21. Capacitance vs. voltage curves measured using $\mathrm{Hg}$ probe method. Hg contact area was $3.1 \times 10^{-3} \mathrm{~cm}^{2}$ .94

Figure 4.22. FTIR characterization of as received CDO (black curve) and thiol (-SH) functionalized $\mathrm{CDO}$ (red curve) 
Figure 4.23. Digital photograph showing ultrasonication setup (a), for qualitatively testing the adhesion strength of $\mathrm{Cu} / \mathrm{Ni} \mathrm{NW}$ s to as received $\mathrm{CDO}$ and thiol modified CDO.....100

Figure 5.1. Schematic diagram showing process flow of $\mathrm{Cu} / \mathrm{Ni} \mathrm{NWs}$ self-assembly into interconnects. .104

Figure 5.2. Optical micrographs of unidirectionally aligned $\mathrm{Cu} / \mathrm{Ni} \mathrm{NWs}$ interconnect channel fabricated depositing $0.5 \mathrm{mg} / \mathrm{mL}$ of NWs solution. 104

Figure 5.3. Schematic diagrams and digital photographs showing interconnect channels fabricated using different types and arrangement styles of NWs

Figure 5.4. Optical micrographs of $\mathrm{Cu}$ NWs based interconnect channel at various magnification scales showing the random arrangement of $\mathrm{Cu}$ NWs .106

Figure 5.5. Optical micrographs of $\mathrm{Cu} / \mathrm{Ni} \mathrm{NWs}$ based interconnect channel at various magnification scales showing directionally well-arranged NWs. .106

Figure 5.6. Optical micrographs of $\mathrm{Cu} / \mathrm{Ni} \mathrm{NWs}$ based interconnect channel at various magnification scales showing randomly arranged NWs

Figure 5.7. Plot showing resistance values of well-aligned and randomly arranged $\mathrm{Cu} / \mathrm{Ni}$ NWs and randomly arranged pure $\mathrm{Cu}$ NWs based interconnect channels. .107

Figure 5.8. Schematic depicting systematic alignment of bar magnets in presence of applied magnetic field and random alignment in absence of magnetic field... .108

Figure 5.9. Optical microscope images of interconnect channels fabricated at various applied magnetic field strength from 0 to 2500 Gauss

Figure 5.10. Plot showing resistance values of $\mathrm{Cu} / \mathrm{Ni}$ NWs based interconnect channels fabricated using various applied magnetic field strength from 0 to $2500 \mathrm{G} \ldots \ldots \ldots \ldots \ldots 110$

Figure 5.11. Low and high magnification optical micrographs showing of interconnect channels fabricated by using $0.5 \mathrm{mg} / \mathrm{mL}, 1.0 \mathrm{mg} / \mathrm{mL}$ and $2 \mathrm{mg} / \mathrm{mL}$ respectively.... .111

Figure 5.12. Resistance values of $\mathrm{Cu} / \mathrm{Ni} \mathrm{NW}$ s based interconnect channels prepared using various concentrations of $\mathrm{Cu} / \mathrm{Ni} \mathrm{NWs}$ solutions .111

Figure 5.13. Thermal stability of well-aligned and randomly arranged $\mathrm{Cu} / \mathrm{Ni} \mathrm{NWs}$ channels and randomly aligned $\mathrm{Cu} / \mathrm{Ni} \mathrm{NWs}$ channels at $100{ }^{\circ} \mathrm{C}$ in air. 


\section{LIST OF SCHEMES}

Scheme 1.1. Schematic representing selective functionalization of surface exposed Si-OH functional groups to thiol-derivatized silicon.................................. 19

Scheme 2.1. Schematic of common reaction pathway for selective functionalization of Si$\mathrm{OH}$ functional groups to Octadecyl and thiol-derivatized silicon...................... 31

Scheme 4.1. Schematic of the new reaction pathway for hydrogen termination and

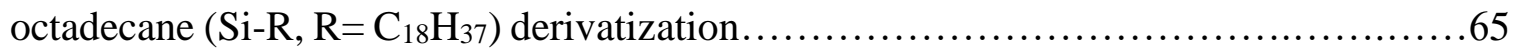

Scheme 4.2. Schematics of various compounds considered for testing surface derivatization reaction....................................................66

Scheme 4.3. Schemes of a) Synthesis of TBDMS Triflate and tert-Butyldimethylsilane, b) Synthesis of tert-Butyltrimethylsilane c) Synthesis of Triphenylsilane................67

Scheme 4.4. Reaction scheme describing thiol functionalization of CDO surface........98 


\section{LIST OF ABBREVIATIONS}

\begin{tabular}{ll} 
Abbreviation & Meaning \\
Cu NWs & Copper nanowires \\
Cu/Ni NWs & Nickel coated copper nanowires \\
SEM & Scanning Electron Microscopy \\
TEM & Transmission Electron Microscopy \\
CA & Contact angle \\
DI & Deionized \\
t-EBSD & Transmitted electron backscattered diffraction \\
EBSD & Electron backscattered diffraction \\
CDO & Carbon doped silicon dioxide \\
CMP & Chemical mechanical polishing \\
DD & Dual Damascene \\
EM & Electromigration \\
PET & Polyethylenetetrapthalate \\
DB & Diffusion barrier layer \\
IC & Integrated circuits \\
RC & Resistance and capacitance \\
FTIR & Fourier transform infrared spectroscopy \\
XPS & X-ray photoelectron spectroscopy \\
XRD & X-ray diffraction spectroscopy \\
EDS/EDX & Energy dispersive X-ray spectrum \\
EDA & Ethylenediamine \\
PECVD & Plasma enhanced chemical vapor deposition \\
OTS & Octadecyltrichlorosilane \\
DIBAL-H & Di isobutyl aluminum hydride \\
& xvi \\
\hline
\end{tabular}




\section{CHAPTER ONE}

\section{INTRODUCTION AND RATIONALE}

Copper is one of the oldest and the third most industrially consumed metal. It has received a great deal of attention for its wide variety of applications, such as electrical conductor in electronics, architecture, plumbing, human health, and many other consumer products $^{1}$. Copper has been the wiring metal of choice in electronic devices because of its ductility, malleability and excellent conductivity characteristics. Especially in chip fabrication, copper is being used as an interconnect material which plays an important role in the power supply and electric signal distribution ${ }^{2}$.

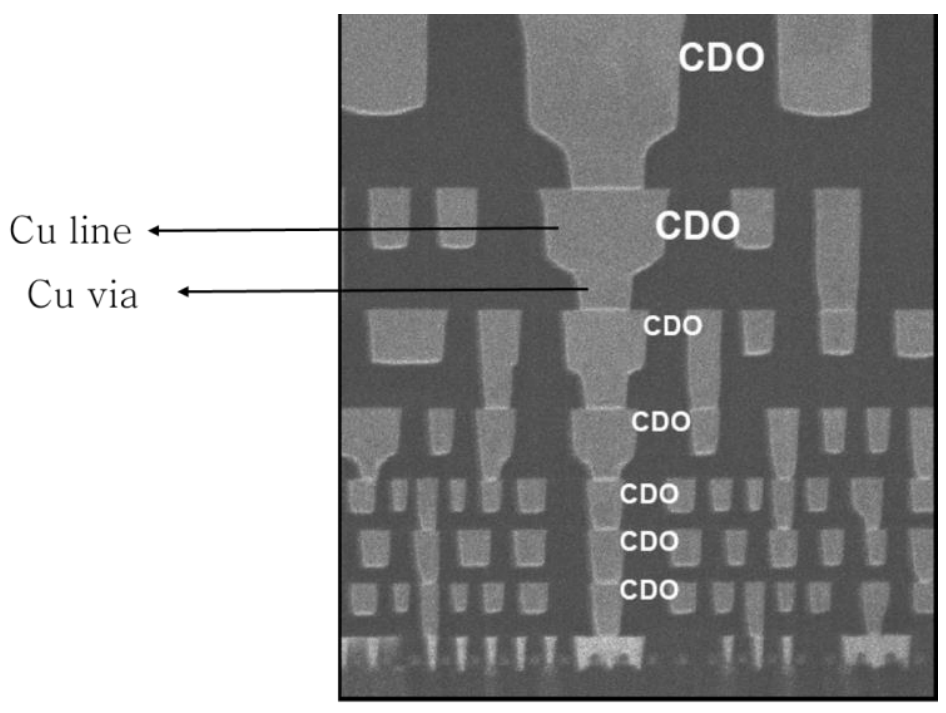

Figure 1.1. Cross section SEM image of copper multilevel interconnect system with CDO as a low- $k$ dielectric ${ }^{3}$ insulator.

In 1997, IBM revolutionized the technology industry with its remarkable innovation of chips made of copper interconnects. Replacing the traditional aluminum with copper as an interconnect material, enabled smaller chip sizes, cheaper production, and faster 
performance ${ }^{4-6}$. Today, in the current ultra large-scale integration (ULSI) technology copper $(\mathrm{Cu})$ and tungsten $(\mathrm{W})$ are being used as interconnect materials. Tungsten is used for ground level interconnects which communicates to the transistors. Copper is being used for all other layers, which can have as many as 12 metallic interconnect levels. Copper metal lines in each level communicate to other levels through copper vias as shown in Figure 1.1.

Ultra large scale integrated (ULSI) circuit device manufacturing technology can be divided into two main stages. The first stage is transistor and its other component structures fabrication. At the second stage, huge numbers of transistors are connected to each other by three-dimensional multilayers of copper interconnect systems ${ }^{3,7}$. Transistors are the active components of a chip and are where signals are controlled and generated. Each transistor communicates to other active parts of a chip through electric signals that are carried by highly complex copper interconnect systems. Ultimate speed limit and chip performance are determined by the frequency at which transistors can turn on and off ${ }^{8}$. Since clock frequency increases with decreasing transistor size, advances in chip speeds have been achieved by shrinking the feature sizes and increasing the count. Currently, the speed limit and performance of a chip is set by signal propagation delays and the time constant of the copper interconnects 9 .

\subsection{Review on traditional process of copper interconnects fabrication on} chips

All metal lines and vias of integrated circuits (ICs) are fabricated by an industrially standard Dual Damascene (DD) process ${ }^{2}$. DD involves defining the line and via pattern in 
a dielectric using photolithography technique and backfilling the opening with copper by an electrochemical method. Finally, the excess copper removed by chemical mechanical planarization (CMP) process $^{2,10}$.

\section{Dual Damascene (DD) process}

The Damascene process was initially developed for jewelry manufacturing ${ }^{2}$. In a single Damascene process trenches and vias are filled with copper in two separate steps. In Dual Damascene (DD) process trench and vias are filled in a single step. In comparison to single Damascene, DD process lowered the number of processing steps and became the industrially standard interconnect fabrication technique. The advantage of this method is super filling, which results in a void-free and seamless filling of high aspect ratio trenches and $\operatorname{vias}^{10}$.

In advanced IC integration, single Damascene is only used for Tungsten (W) local interconnects and all other copper interconnects are made by using Dual Damascene process. All high-density metal lines and vias are insulated by an inner layer dielectric (ILD) material. Copper is known to be a fast diffuser into silicon and its oxide dielectrics. This causes deep level defects which may lead to poor performance or chip failure. By using a Ti, TiN, Ta or TaN as a diffusion barrier, one can localize copper to where it is desired $^{2}$. 

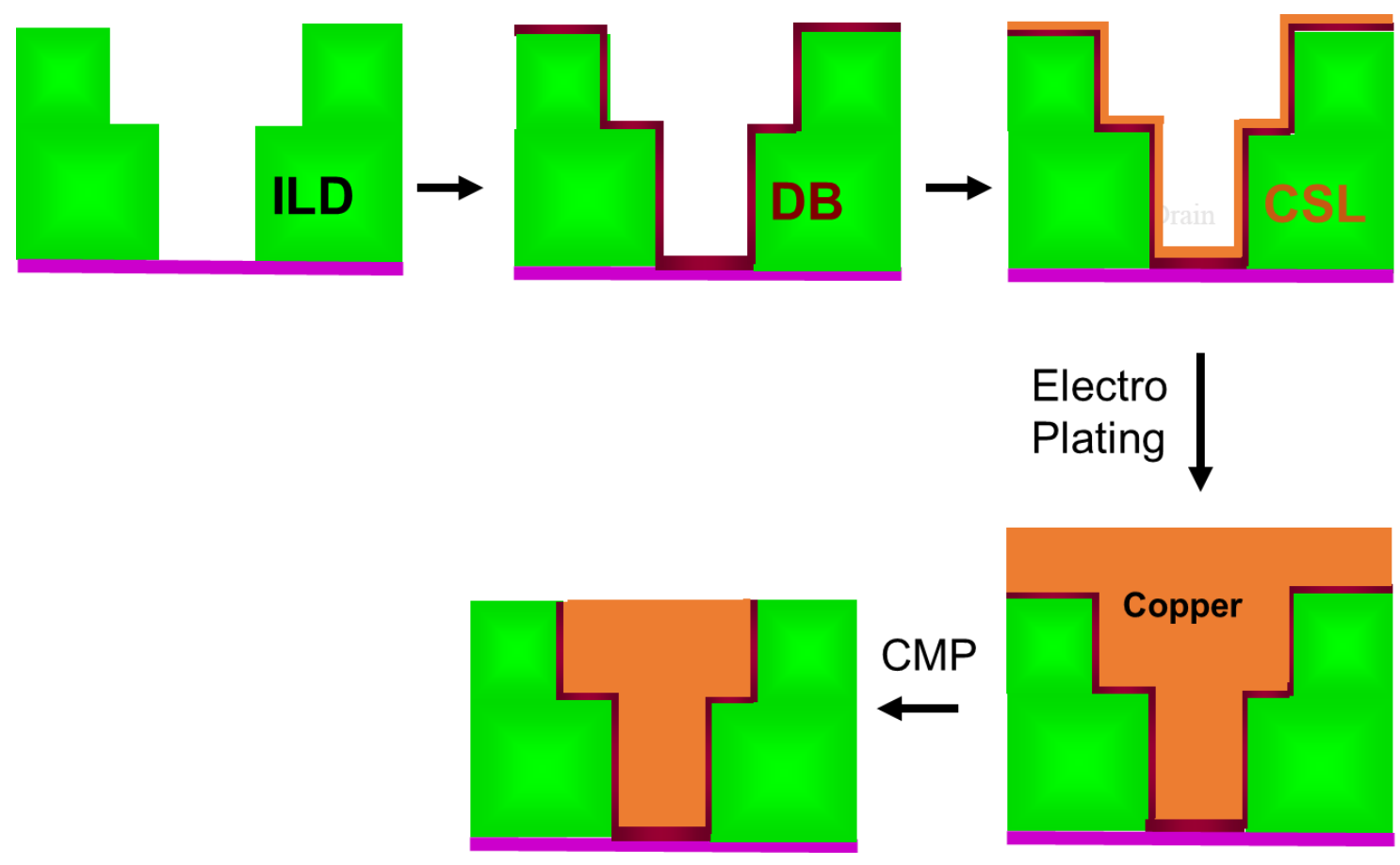

Figure 1.2. Schematic of DD process for making metal lines and vias. The process involves diffusion barrier layer (DB) deposition, copper seed layer deposition (CSL), copper filling by electroplating process and removal of excess copper by a chemical mechanical planarization process $(\mathrm{CMP})^{2}$.

Major steps involved in DD process are illustrated in Figure 1.2. The DD process starts with depositing a thin layer of a diffusion barrier (DB) layer (Ta, TaN) commonly done by ionized metal plasma (IMP) method. It is followed by depositing a thin copper seed layer (CSL), usually by sputtering. Copper electroplating method emerged as the most promising and commercial viable technique for copper filling ${ }^{8}$. A schematic of electrochemical deposition set up is shown in Figure $1.3^{8}$. CSL coated wafer is then immersed in an electroplating solution and electrically connected and acts as a cathode. Copper sulfate $\left(\mathrm{CuSO}_{4}\right)$ and sulfuric acid $\left(\mathrm{H}_{2} \mathrm{SO}_{4}\right)$ are the common components of 
electrolytes. Cupric ions $\left(\mathrm{Cu}^{2+}\right)$ from an electrolyte solution get reduced and deposited on CSL surface which results in super filling of trenches and vias.

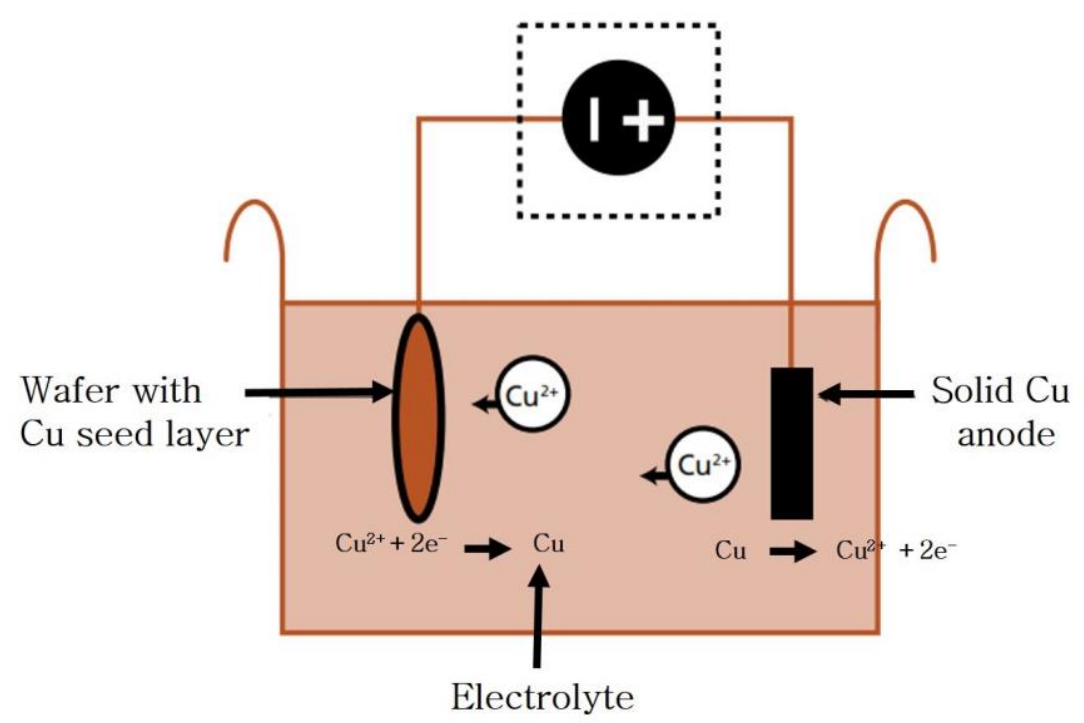

Figure 1.3. Schematic diagram of electroplating of copper on the copper seed layer (CSL) coated wafer ${ }^{8}$.

After trenches and vias are filled with copper, the excess copper is removed by Chemical Mechanical Polishing (CMP). In CMP process, polishing with a slurry removes excess copper by the combination of chemical reactivity and mechanical abrasion ${ }^{2,10}$. Chemicals in the slurry react with copper and typically form copper oxides and are subsequently removed by mechanical abrasion. After polishing for an optimum period, the wafer surface becomes planar. Capping of $\mathrm{Cu}$ interconnects by silicon nitride, or silicon carbide (known as Etch Stop (ES) layer) completes one level of interconnect fabrication. All these above discussed processes will be repeated for multiple times for the fabrication of multilayered highly complex $\mathrm{Cu}$ interconnects. 


\subsection{Challenges in modern integrated circuits fabrication}

Today, modern chips use several billion of transistors and other electronic components in an area of about $1 \mathrm{~cm}^{211}$. As the transistor count increasing, copper wire dimensions are shrinking to nanometer scales to connect these individual transistors.

With the ever-decreasing copper wire dimensions, the resistance of the narrow conductive channel is increasing due to electron scattering by the grain boundaries and at wire surfaces. Increased resistance adversely contributes to the overall signal propagation delay. In the modern chips, total resistance $(\mathrm{R})$ of the copper interconnect structure is a significant factor that affects the chips performance ${ }^{9,11}$. Furthermore, as the area of copper interconnects increase, the capacitance (C) between the closely squeezed conductive channels also increases. Both effects significantly increase in the RC signal delay, which results in degraded performance of the chips ${ }^{9}$. Hence, in recent years enormous efforts have been devoted to developing new process technologies to fabricate miniaturized crystalline copper interconnects and to prepare low- $k$ insulators to minimize the capacitance (C) and leakage current.

In the narrow copper wires, mechanical stress and current density significantly increase $\left(>2 \mathrm{MA} / \mathrm{cm}^{2}\right)$ which ultimately leads to electromigration (EM) related interconnect failures ${ }^{12,13}$. High current density in the narrow metal lines causes void nucleation, propagation along the metal lines and creates cracks ${ }^{14}$. Electromigration failure mechanisms have been discussed in detail elsewhere ${ }^{13,15,16}$. Also, the standard DD process requires deposition of diffusion barrier and copper seed layers in the narrow trenches and vias. But, as the feature sizes are shrinking to few nanometer scales, it is becoming very 
challenging to have these layers and fill them with copper. Ultimately, the fabrication of interconnects in a DD process is reaching its fundamental limits. Therefore, innovative materials and process solutions are very critical to sustaining the miniaturization and performance trends.

\subsection{Alternate materials and methods for interconnect fabrication}

In the recent history, bottom-up or hybrid approaches of nanoscale circuit fabrication using nanomaterials as building blocks, have received considerable attention ${ }^{17-19}$. This kind of bottom-up approach of integrated circuit fabrication involves the synthesis of nanocrystals with desired physical and electrical properties and their self-assembly into electronic circuits ${ }^{19}$.

\section{Desirable material properties for the fabrication of interconnects:}

As the DD processed on-chip copper wire dimensions are reaching to nanoscales, they experience two principal issues. First, electron scattering induced degradation of electrical resistivity. Second, their reliability issues due to poor electromigration (EM) resistance ${ }^{17}$. In addition to the above two issues, oxidation under ambient conditions and enhanced oxidation at high resistive self-heating are also the major growing concerns ${ }^{20}$.

The ideal building blocks used for the interconnect fabrication should possess high conductivity, excellent electromigration (EM) resistance, high oxidation resistance and economical $^{20}$. Therefore, a quest for alternate interconnect materials which address the above issues have been under active search. 


\section{Promising building blocks for the fabrication of interconnects:}

Nanomaterials show significantly improved electrical, mechanical and thermal properties compared to their bulk material counterparts. Especially 1D nanomaterials, such as pure metallic nanowires, core-shell nanowires and carbon nanotubes (CNT), etc., are very attractive building blocks for functional nanoscale electronic devices, owing to its unique anisotropic structure-dependent properties. Assembly of 1D nanomaterials in a bottom-up or hybrid approach offers an excellent alternative for the fabrication of miniaturized and high-performance interconnects ${ }^{17,20-28}$. Many kinds of 1D nanomaterials such as pure metallic $\left(\mathrm{Cu}^{17,}{ }^{29}, \mathrm{Ag}^{20}, \mathrm{Au}^{25}, \mathrm{Pt}^{30}\right) \mathrm{NWs}$, core-shell NWs (Graphene encapsulated $\mathrm{Cu} \mathrm{NWs}^{31}$ ) and carbon nanotubes ${ }^{32}(\mathrm{CNT})$, etc., have been explored as promising building blocks for interconnects ${ }^{17}, 20-28$.

Among all 1D nanomaterials, copper nanowires (Cu NWs) have attracted considerable interest in recent years due to their intriguing electrical, thermal and mechanical properties ${ }^{17,18,33}$. Copper has the second highest electrical conductivity among metals, highly abundant and low cost. Thus $\mathrm{Cu}$ NWs have been considered as promising building blocks for many applications, such as flexible \& transparent electrodes ${ }^{33-35}$, conductive inks ${ }^{18}$, catalyst ${ }^{36}$ and interconnects ${ }^{17}$. Also, copper is successfully being used as an interconnect material on-chip since $1997^{37}$. Therefore, $\mathrm{Cu}$ NWs are the ideal candidates for many micro and functional nanoscale devices, and it is imperative to develop a simple and reliable technique to assemble the $\mathrm{Cu}$ NWs into a functional device. 


\section{Review of NW self-assembly techniques:}

Self-assembly refers to the process in which nanowires or other discrete components spontaneously organize into ordered macroscopic structures owing to its direct specific interactions (e.g., interparticle forces), or indirectly using an externally applied field ${ }^{30,38 \text {, }}$ 39. The essential feature of nanowire self-assembly is an organization of NWs in an orientationally and positionally well-ordered manner ${ }^{30,40}$.

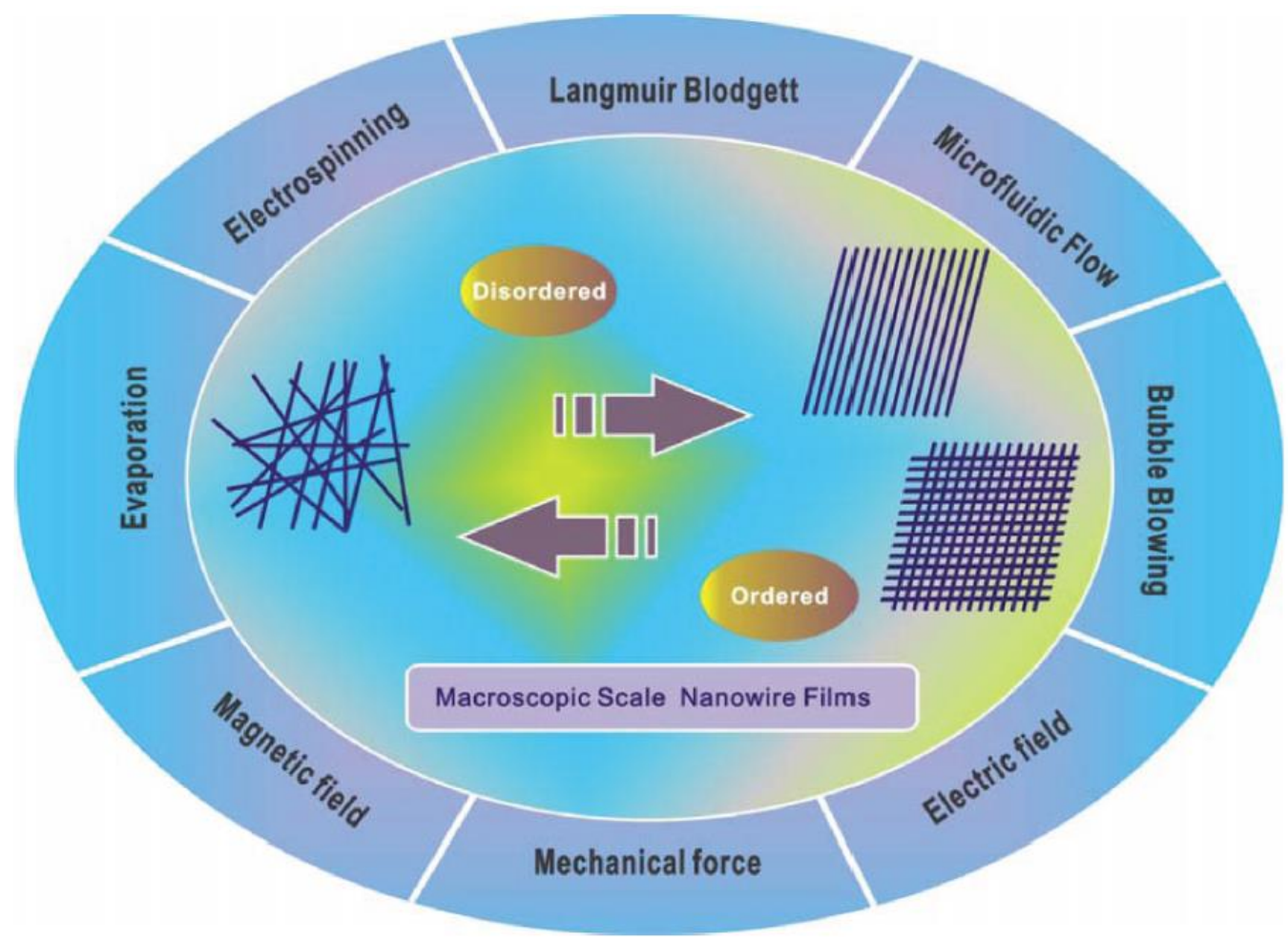

Figure 1.4. Schematic describing various kinds of nanowires self-assembly techniques that arranges disordered NWs into well-ordered manner ${ }^{41}$.

In a bottom-up or hybrid approach, precisely controlling the NWs orientation and positioning are the first critical steps for the successful assembly of individual NWs into a functional nanodevice ${ }^{38,42,43}$. Several approaches for controlling the orientation of NWs via a self-assembly process have been studied (See Figure 1.4), such as Langmuir- 
Blodgett ${ }^{44}$, microfluidic flow ${ }^{38}$, evaporation induced ${ }^{38}$, nematic liquid crystal phase ${ }^{40}$, shear induced ${ }^{45}$, mechanical force ${ }^{38}$, electrospinning ${ }^{41,}{ }^{46}$, electric ${ }^{47}$ and magnetic field assisted techniques ${ }^{30,38,41-43,45,48,49}$.

The Langmuir-Blodgett technique involves compression of NW suspensions at airwater interface into dense films ${ }^{44}$. During the compression, NWs organize themselves into the well-aligned and closely packed structure. And, dip coating process can then be used for transferring the well-aligned NWs on to a solid substrate. However, the primary challenge in using this approach is, during the dip coating process reorganization of the NWs results in overlapping features and gaps within the dense arrays of $\mathrm{NWs}^{38,44}$. Evaporation-induced and microfluidic channel assisted alignment techniques involves, reorientation of NWs into well-ordered dense arrays with the direction of fluid flow to minimize the fluid $\mathrm{drag}^{38}$. Confining the fluid flow to the microfluidic channel enhances the shear force and results in more uniform alignment. However, it is hard to control the alignment uniformity in the case of the evaporation induced technique, and the requirement of fabrication of microfluidic channels limits the wafer-scale assembly of NWs using these techniques ${ }^{38}$.

Nematic liquid crystal phase technique depends on the volume fraction of nanowires or nanorods in a given solvent. In a very dilute solution, NWs can rotate or translate freely under Brownian motion without interference from another NW/rod. But, as the concentration increases to a critical concentration $\left(\Phi_{\mathrm{N}}\right)$, a nematic liquid crystal phase forms and NWs organize themselves into a densely packed and orientationally ordered manner ${ }^{40}$. However, the special requirements like monodispersity, high aspect ratio 
nanowires and that NWs should not precipitate out from the solution, etc. limits this technique.

Shear force or mechanical force induced reorganization of NWs is one of the largescale assembly techniques ${ }^{38,45}$. Here, a shear force is created by the motion of a solid object or fluid against a second solid object or fluid. In either case, a force is generated parallel to the sliding direction and aligns the NWs in the direction of movement. This technique is inexpensive and can be applied to various substrates such as $\mathrm{Si}$ wafers and flexible or curved substrates. However, this process requires excellent skills and the challenging thing in this technique is controlling the viscosity of the NWs solution ${ }^{38}$.

Electrospinning is a versatile technique, using electrostatic forces to form continuous chains of fibers, polymers or nanowires. In a typical electrospinning technique, NWs or a polymer suspension pumped through a thin nozzle with an inner diameter of $\sim 100 \mu \mathrm{m}^{41,46}$. And, the nozzle simultaneously serves as an electrode, to which high electric field of 100$150 \mathrm{kV} \mathrm{m}^{-1}$ is applied. While the NWs solution is passing through the nozzle, under the influence of the strong electrostatic field, assembly of electrospinning fibers along the axial directions occurs. Very complex experimental setup and the requirement of high voltages are limits to the usefulness of this technique.

In the case of electric field assisted alignment technique, highly polarizable NWs polarize readily in the alternating electric field due to charge separation at the NWs surface and will experience the dielectrophoretic force that produces net movement to the $\mathrm{NWs}^{47}$. However, NWs at various locations in between the electrodes experience an unequal dielectrophoretic force which results in poor alignment in the center regions and excellent 
alignment in the regions close to the electrodes. However, the requirement of electrode fabrication for the NWs alignment and it is only applicable to the polarizable materials (e.g., $\mathrm{CdSe}, \mathrm{ZnO}, \mathrm{Se} \mathrm{NWs}$ ), etc., limits this technique to implementation for large-scale assembly processes.

Finally, the magnetic field assisted technique involves applying of an external magnetic field to directionally organize the magnetically active nanowires suspended in the solution ${ }^{30,38,41-43,45,48-50}$. In the presence of an applied magnetic field, each NW will polarize into north and south poles and orient themselves parallel to the applied magnetic

field direction ${ }^{30,51}$. The uniformity of the NWs alignment depends on the applied magnetic field strength. The added advantage of this technique is the assembled NWs forms chainlike structures by connecting each NW in a head to tail fashion, which gives a continuous network of NWs. These kinds of continuous networks are ideal for the best electrical performance. However, the main drawback of this technique is, it can only be applied to magnetically active NWs.

\section{Review on using nanowires self-assembly techniques to build functional nanodevices:}

The number of nanodevices, such as interconnects, FETs, bipolar transistors, light emitting diodes (LED) and logic gates have been assembled in a bottom-up approach from welldefined nanowires ${ }^{17}, 29,30$. Here discussed are few NWs self-assembly techniques used for assembling the NWs into functional devices. 


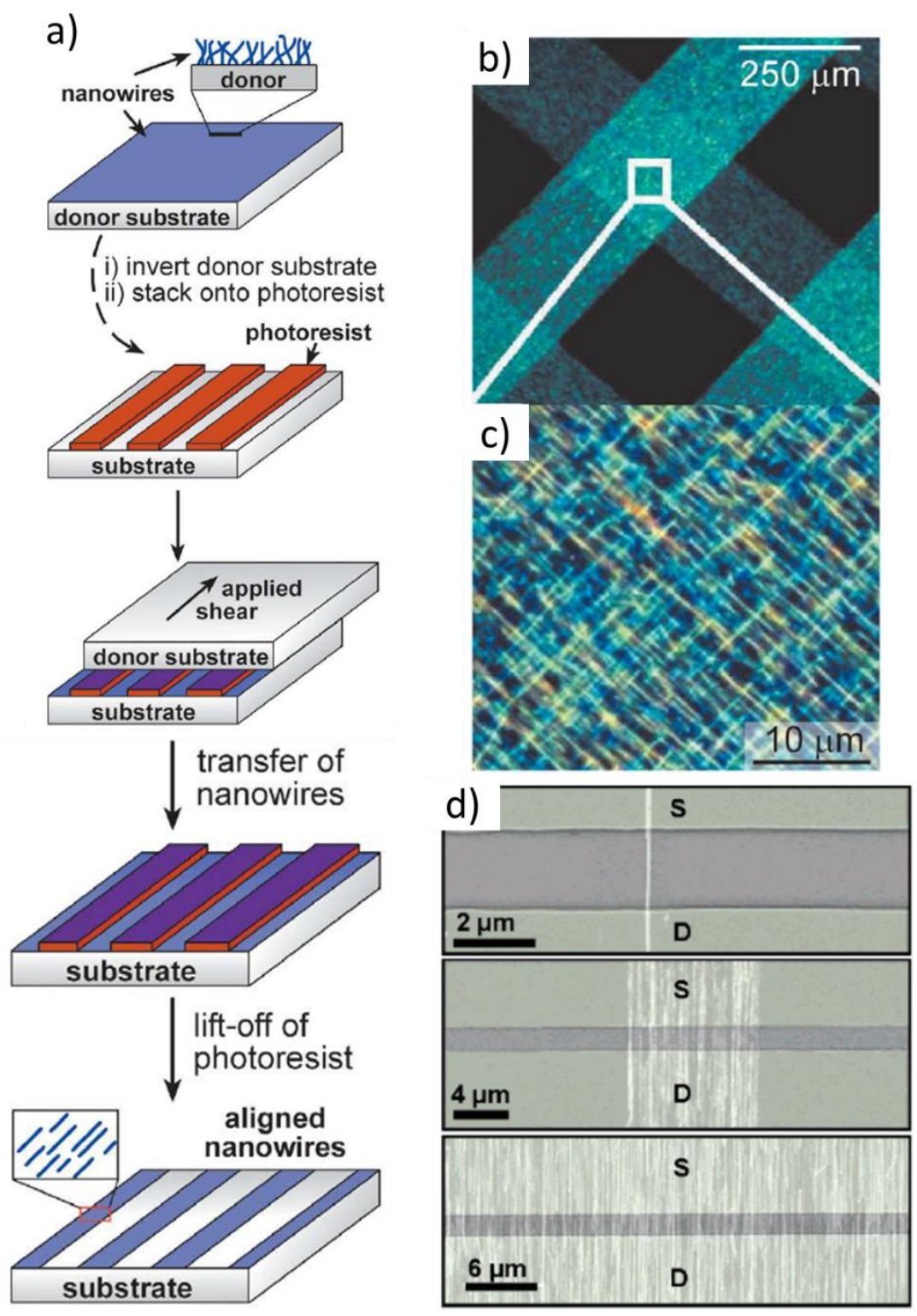

Figure 1.5. Schematic diagram showing shear force induced self-assembly of Ge NWs into photolithography patterned channels (a), and optical micrographs of crossed arrays of Ge NWs shown at low (b) and high (c) magnifications. SEM images of single and multiple Ge NWs assembled into FET device (d $)^{38,52}$.

Figure 1.5 depicts the shear force assisted NWs self-assembly into crossed arrays and field effect transistor (FET) device. This process involves, mechanical movement of a donor substrate consisting of a lawn of vertically grown NWs, onto a photolithography 
patterned receiver substrate. This mechanically generated force can direct the NWs to align in a unidirectional manner, and the repetition of sliding in an orthogonal direction produces crossed arrays of NWs. The same shear force induced technique was used to fabricate single or multiple NWs based FET devices. This is an excellent wafer scale assembly technique; however, the limitation is this process requires vertically grown NWs which are usually prepared by complex CVD or template assisted processes ${ }^{53,54}$.
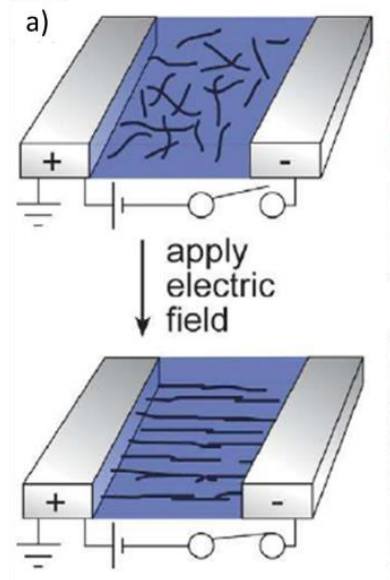
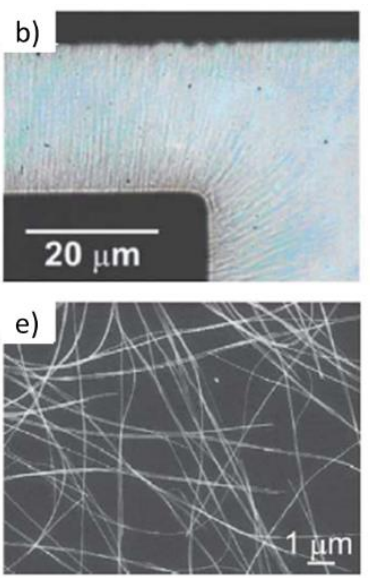
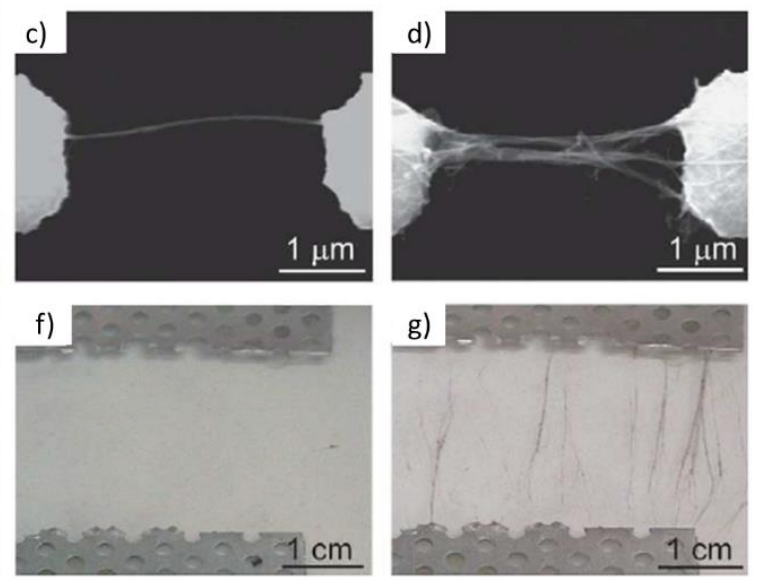

Figure 1.6. Schematic illustrating electric field assisted alignment of nanowires (a). Dense arrays of cadmium selenide nanowires assembled near the electrode (b). SEM images (c, d) zinc oxide nanowires assembled between electrodes. (e-g) SEM and optical microscope images of flexible selenium nanowires before and after $(\mathrm{g})$ assembly ${ }^{38,55}$.

When a highly polarizable, but uncharged nanowire (e.g., CdSe, ZnO, Se NWs) is subjected to a reasonably strong ac electric field $(>1 \mathrm{kV} / \mathrm{cm})$, an induced dipole moment is created enabling the nanowire to respond to the electric field gradient ${ }^{55}$. The NWs will reorient in the lowest energy configuration, such as parallel to the applied electric field. This process involves deposition of an NWs solution in between the electrodes and allowing it to dry in the presence of an applied electric field. Figure 1.6 shows optical and 
SEM images of self-assembled nanowires near or in between the electrodes. However, this process is only applicable to polarizable NWs, and requirements such as microelectrode fabrication and high voltage limits this technique.

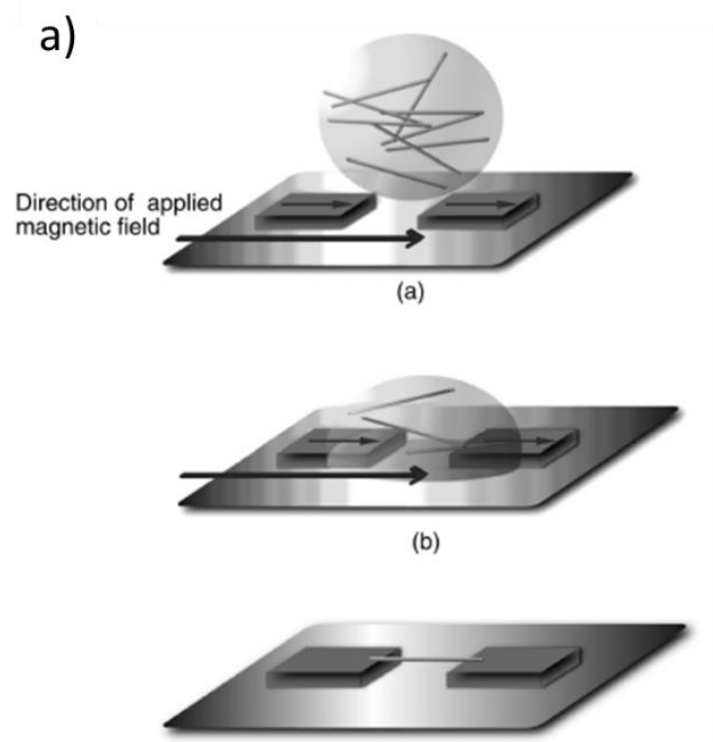

(c)

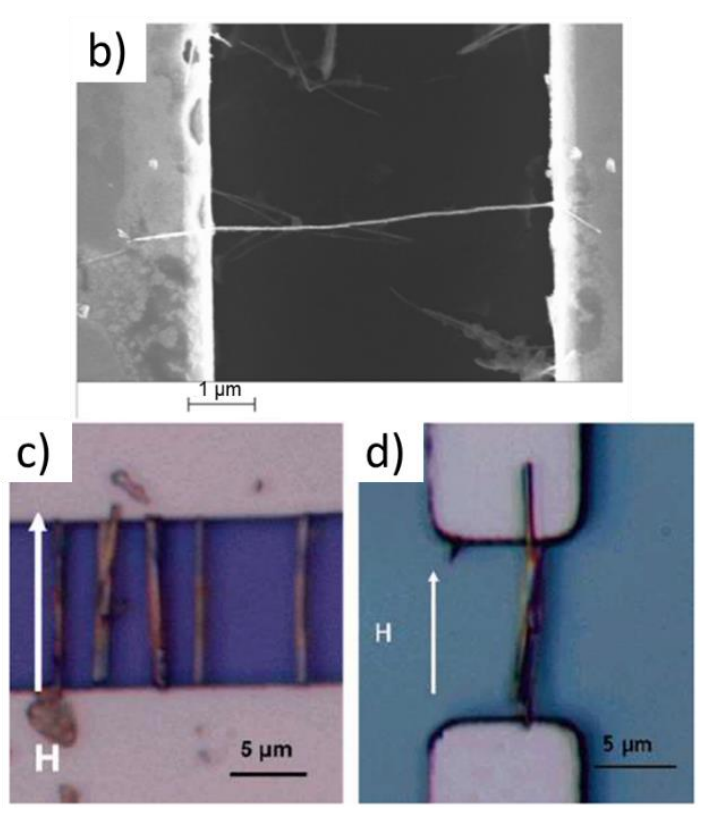

Figure 1.7. Schematic diagram illustrating the process of magnetic field assisted assembly of nickel (Ni) NW interconnect on Ni electrodes (a). SEM image of as-fabricated Ni NW based interconnect $(b)^{56}$. Optical microscope images of $\mathrm{Ni} / \mathrm{Au} / \mathrm{Ni} \mathrm{NWs}$ based interconnects ${ }^{51}$.

Several efforts have been devoted to the magnetic field assisted self-assembly of magnetically active nanowires ${ }^{40-42,} 44,47,48$. The magnetic field assisted self-assembly involves directed deposition of well-aligned NWs on the electrodes. This process involves deposition of suspension of a magnetically active NWs solution on substrate and drying of the solution in the presence of applied magnetic field like the schematic shown in Figure 1.7. In the solution, the NWs align in the applied magnetic field direction, and the orientationally aligned NWs settles down on the electrodes ${ }^{50,56}$. Self-assembly of single and multiple NWs based interconnects have been fabricated using pure $\mathrm{Ni} \mathrm{NWs}^{56}$, 
$\mathrm{Ni} / \mathrm{Au} / \mathrm{Ni} \mathrm{NWs}^{51}, \mathrm{Au} / \mathrm{Ni} / \mathrm{Au}^{50}$ nanowires. However, to date, the magnetic field assisted self-assembly technique is limited to assemble the single or few NWs into a nanodevice but, assembly of NWs on a large-scale has not yet been demonstrated.

This thesis work describes an investigation of magnetic field assisted self-assembly of high aspect ratio nickel coated copper nanowires ( $\mathrm{Cu} / \mathrm{Ni} \mathrm{NWs})$ into photolithography patterned channels on a large-scale area.

\subsection{Thesis objectives and outlines}

The overall objective of the thesis is to self-assemble one-dimensional (1D) nanocrystalbased interconnects. The central hypothesis of the project is "interconnects on IC surfaces can be self-assembled." This hypothesis was tested by developing a magnetic field assisted technique to self-assemble the $\mathrm{Cu} / \mathrm{Ni} \mathrm{NWs}$ into photolithography patterned channels on the large-scale area on a carbon doped silicon dioxide (CDO) wafer, like the figure shown in Figure 1.8.

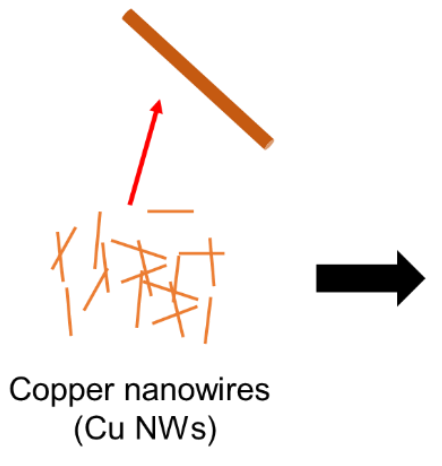

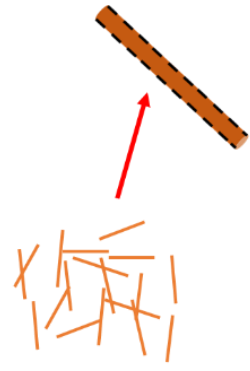

Nickel coated Copper nanowires (Cu/Ni NWs)

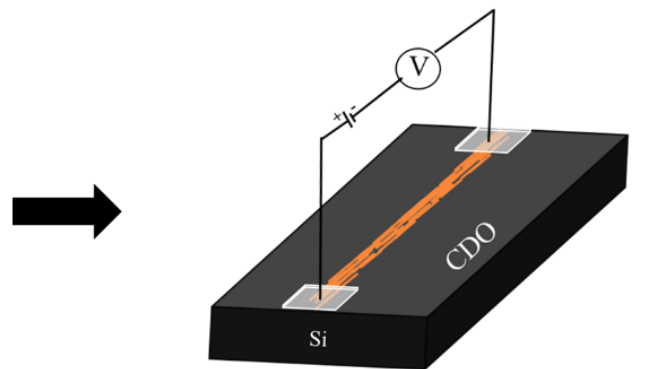

$\mathrm{Cu} / \mathrm{Ni}$ NWs self-assembled in patterned channel

Figure 1.8. Schematic representing self-assembly of $\mathrm{Cu} / \mathrm{Ni} \mathrm{NWs}$ into photolithography patterned channels on CDO wafer. 
Nanowires self-assembly was achieved via three specific aims. Aim \#1 is Cu NWs synthesis and electroless coating of nickel onto the Cu NWs surface. Aim \#2 is a preparation of the $\mathrm{CDO}$ surface to accept nickel coated copper nanowires ( $\mathrm{Cu} / \mathrm{Ni} \mathrm{NWs})$. Aim \#3 is magnetic field assisted self-assembly and deposition of NWs into photolithography patterned channels.

\section{Aim \#1: Cu NWs synthesis and electroless coating of nickel onto the Cu NWs surface:}

In the fabrication of future bottom-up nanotechnology, the synthesis of copper nanowires $(\mathrm{Cu} \mathrm{NWs})$ has received considerable attention in recent years, and a number of methods have been successfully demonstrated which include template assisted processes ${ }^{26}$, chemical vapor depositions ${ }^{53}$, hydrothermal/solvothermal routes $^{57}$, and solution-based methods $^{33,35,58}$. Among all the available synthesis routes, the solution-based approach is very attractive due to its process simplicity, low cost and easy to scale up for bulk scale production $^{58}$. However, a thorough understanding of the effect of reagent concentrations and synthesis conditions on the morphology, shape and aspect ratio of NWs are very critical. This study focuses on the effect of reducing agent concentration and synthesis temperature on the resulting surface morphology and aspect ratio of $\mathrm{Cu}$ NWs. The optimum reagent conditions to synthesize high aspect ratio $\mathrm{Cu}$ NWs with a smooth surface are to be established.

Although applications of $\mathrm{Cu}$ NWs have been demonstrated in a wide range of devices (e.g., transparent and flexible electrodes and conductive ink, etc.), oxidation of pure copper into copper oxides limit its reliable performance ${ }^{59}$. Also, very limited success has been achieved in controlling the orientation and positioning of $\mathrm{Cu}$ NWs, which is very critical 
for assembly of these NWs into complex functional devices. The above two challenges are addressed by coating a thin layer of oxidation resistant and ferromagnetic nickel onto the $\mathrm{Cu}$ NWs surface in an electroless coating approach. Nickel coating helped in protecting the copper core from oxidation and gave the magnetic response to $\mathrm{Cu}$ NWs. The orientation of $\mathrm{Cu} / \mathrm{Ni} \mathrm{NWs}$ is controlled by applying the uniform magnetic field to the NWs solution deposited onto the CDO surface.

\section{Aim \#2: Preparation of CDO surface to accept nickel coated copper nanowires $(\mathrm{Cu} / \mathrm{Ni}$ NWs).}

$\mathrm{CDO}$ is a low- $k$ dielectric material composed of $\mathrm{Si}, \mathrm{C}, \mathrm{O}, \mathrm{H}$ elements and contains surface exposed hydrophilic Si-OH functional groups. A CDO material is chosen to demonstrate the NWs self-assembly process ${ }^{60-62}$. Modern interconnects utilize this material to separate copper wiring in interconnects. It is well known that thiol (-SH) functional groups show strong interaction towards copper and nickel. Therefore, this work uses a thiol-functionalized $\mathrm{CDO}$ surface to attract and hold the $\mathrm{Cu} / \mathrm{Ni}$ NWs strongly after deposition. Thiol functionalization of a CDO surface is achieved via a novel reaction scheme developed to selectively functionalize the surface exposed Si-OH functional groups to thiol-terminated alkyl groups, deploying the scheme shown in Scheme 1.1. The reaction process involves the conversion of hydrophilic $\mathrm{Si}-\mathrm{OH}$ functional groups to its triflate ester (Si-O-Tf), and it is followed by reduction to hydrogen-terminated silicon ( $\mathrm{Si}$ H) using DIBAL-H reagent. Finally, hydrosilylation of Si-H using 2-propene thiol generates the thiol-functionalized CDO surface. 


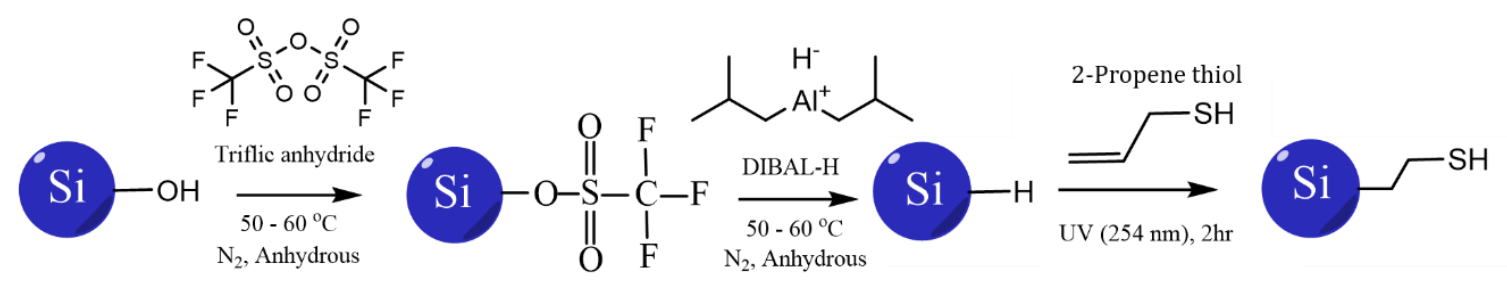

Scheme 1.1. Schematic representing selective functionalization of surface exposed Si-OH functional groups to thiol (-SH) terminated surface on CDO.

\section{Aim \#3: Magnetic field assisted self-assembly of NWs into photolithography patterned channels}

$\mathrm{Cu} / \mathrm{Ni} \mathrm{NW}$ s self-assembly into a photolithography patterned channel is demonstrated by using the combination of photolithography, and magnetic field assisted NWs orientation controlling techniques as shown in Figure 1.8. Photolithography is used to define the circuit pattern (300 $\mu \mathrm{m}$ width and $4 \mathrm{~mm}$ length) on a thiol-functionalized CDO wafer. And, the orientation of $\mathrm{Cu} / \mathrm{Ni} \mathrm{NWs}$ is controlled by applying the uniform magnetic field to the $\mathrm{Cu} / \mathrm{Ni}$ NWs dispersed aqueous solution. When the solution is dried, orientationally well aligned NWs will deposit on the photolithography patterned CDO substrate like the image shown in Figure 1.9. Finally, acetone solvent is used to dissolve the photoresist, and it simultaneously washes off the loosely bound NWs from the photoresist surface and ultimately leaves the self-assembled NWs channel on the CDO substrate. Electrical characterization is performed by measuring the resistance of the as-fabricated NWs based interconnect channel. 


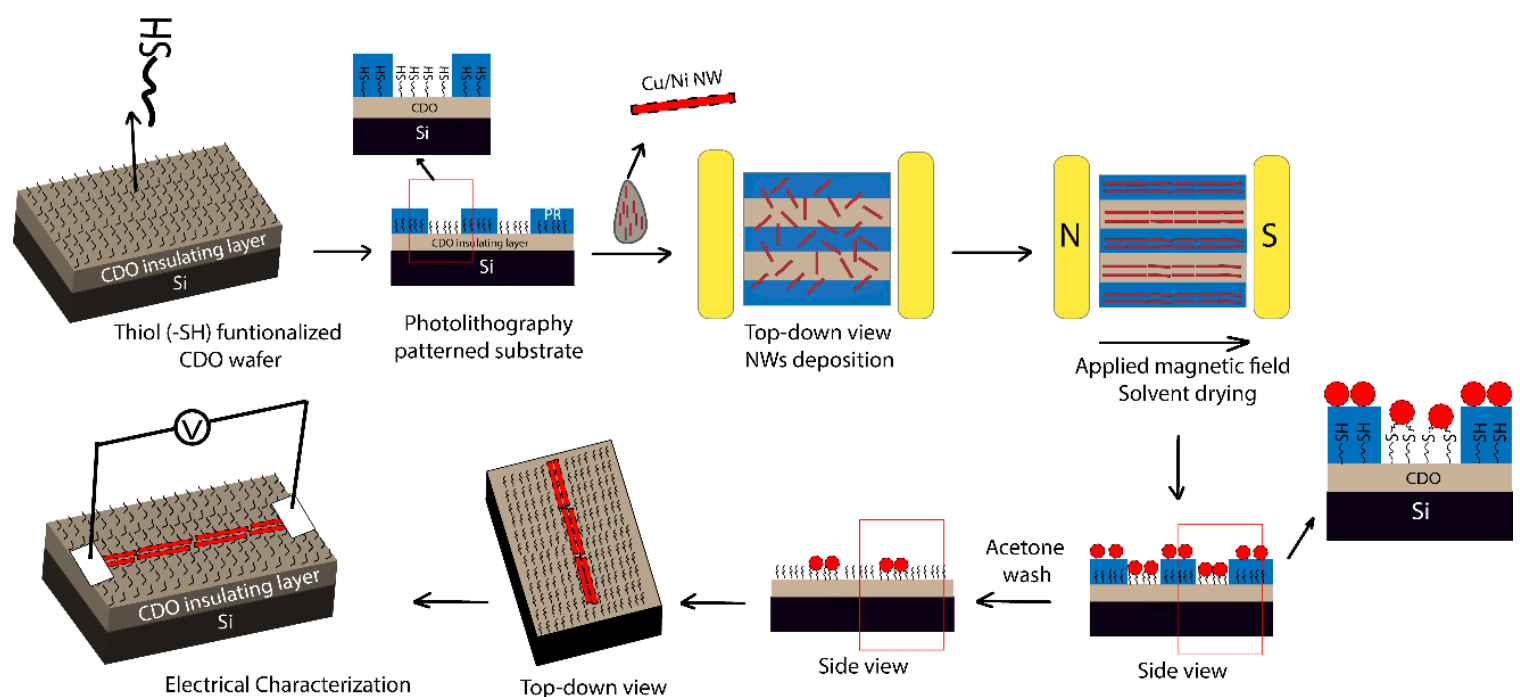

Figure 1.9. Schematic diagram showing a process flow of $\mathrm{Cu} / \mathrm{Ni} \mathrm{NWs}$ self-assembly into interconnect channels.

\section{Thesis Outlines:}

The following chapters provide the detailed information about results collected to support the above-discussed hypothesis. Chapter-2 discusses instrumentation, materials, and synthesis methods used in this study. The results of copper nanowires synthesis, an electroless coating of nickel onto $\mathrm{Cu}$ NWs surface and magnetic field assisted NWs orientation controlling technique details are discussed in Chapter-3. Selective functionalization of surface exposed hydrophilic $\mathrm{Si}-\mathrm{OH}$ functional groups to thiol (-SH) terminated surface results are discussed in Chapter-4. And, $\mathrm{Cu} / \mathrm{Ni}$ NWs self-assembly into photolithography patterned channels and electrical characterization results are discussed in Chapter-5. The thesis concludes with the discussion of summary, limitations, other applications and future scope of the developed techniques in Chapter-6. 


\section{CHAPTER TWO}

\section{INSTRUMENTATION, MATERIALS AND METHODS}

\subsection{INSTRUMENTATION}

\subsubsection{FTIR Spectroscopy}

Structural and functional group information on Silica gel, $\mathrm{SiO}_{\mathrm{x}}$, and $\mathrm{CDO}$ substrates were studied by Fourier transform infrared (FTIR) spectroscopy. The infrared spectrum is a nondestructive and powerful identification tool for functional groups in a wide range of samples (liquid, gel, solid substrates). FTIR data was collected on Thermoscientific, Nicolet ${ }^{\mathrm{TM}}$ FTIR Spectrometer, in transmittance mode from 400 to $4000 \mathrm{~cm}^{-1}$ at a resolution of $4 \mathrm{~cm}^{-1}$. For collecting FT-IR spectra of silica gel samples, a small amount of sample ( $5 \mathrm{wt} \%$ ) was mixed with $\mathrm{KBr}$ salt and pressed into a thin pellet. $\mathrm{CDO}$ or $\mathrm{SiO}_{\mathrm{x}}$ wafers (dimension 1x2 cm) were directly placed in the path of IR beam and spectra collected in transmission mode.

\subsubsection{NMR Spectroscopy}

Nuclear magnetic resonance (NMR) spectroscopy was used in structural characterization and reaction progress study of organic model compounds. ${ }^{1} \mathrm{H}$ and ${ }^{19} \mathrm{~F}-$ NMR spectra were collected on a $400 \mathrm{MHz}$ Bruker NMR instrument at 256 scans. Reaction mixtures were dissolved in $\mathrm{C}_{6} \mathrm{D}_{6}$ or DMSO- $\mathrm{d}_{6}$ or $\mathrm{CDCl}_{3}$ solvents. In all discussed reactions percentage yields were calculated from ${ }^{1} \mathrm{H}$ or ${ }^{19} \mathrm{~F}-\mathrm{NMR}$ spectra of crude mixtures.

\subsubsection{X-ray Photoelectron Spectroscopy (XPS)}


Surface chemical composition and bonding information were investigated using XPS. It is a qualitative and quantitative technique for analyzing surface chemistry of materials with extremely high sensitivity. XPS spectra were acquired on PHI Versa Probe II Scanning XPS equipped with Argon (Ar), $\mathrm{C}_{60}$ sputtering gun and dual charge neutralizer. Survey spectrums were collected at $187.85 \mathrm{eV}$ pass energy, $1 \mathrm{eV} / \mathrm{step}, 20 \mathrm{~ms}$ per step, 5 cycles and 5 sweeps. And, high-resolution spectra were collected at $23.5 \mathrm{eV}$ pass energy, $0.1 \mathrm{eV} / \mathrm{step}, 20 \mathrm{~ms}$ per step, 5 cycles and 5 sweeps. PHI MultiPak data reduction program was used for the deconvolution and analysis of raw XPS data.

\subsubsection{Contact angle measurements}

The contact angle (CA) refers to the angle where a liquid droplet interacts with a flat and horizontal solid surface. It quantifies the wettability of the surface by the liquid droplet. A low contact angle (flat droplet) for a water drop indicates that the surface has high wettability (hydrophilic) and a high contact angle indicates the poor wettability (hydrophobic), see Figure 2.1b.

Water contact angle data was collected in a laboratory fabricated setup, like the schematic and digital photograph shown in Figure 2.1 a \& c. For the data collection, a PC connected to a Kodak MDS 100 CCD camera was used to capture the droplet images on the surface of a wafer substrate. The contact angle data was analyzed by using Image-J software application (freeware available from $\mathrm{NIH}$ ). 


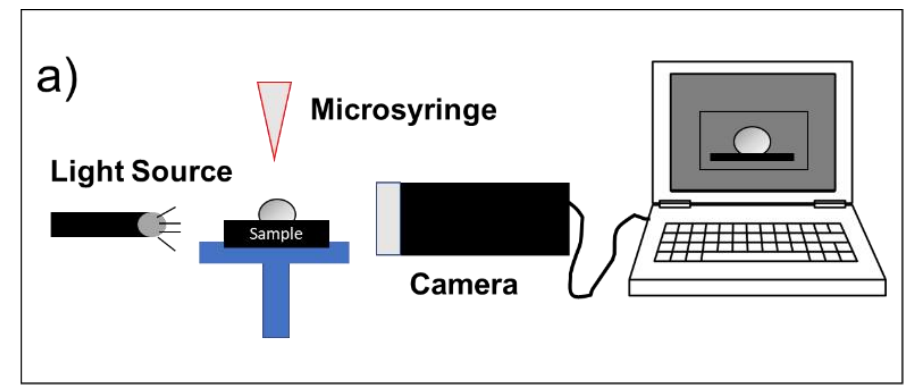

b)
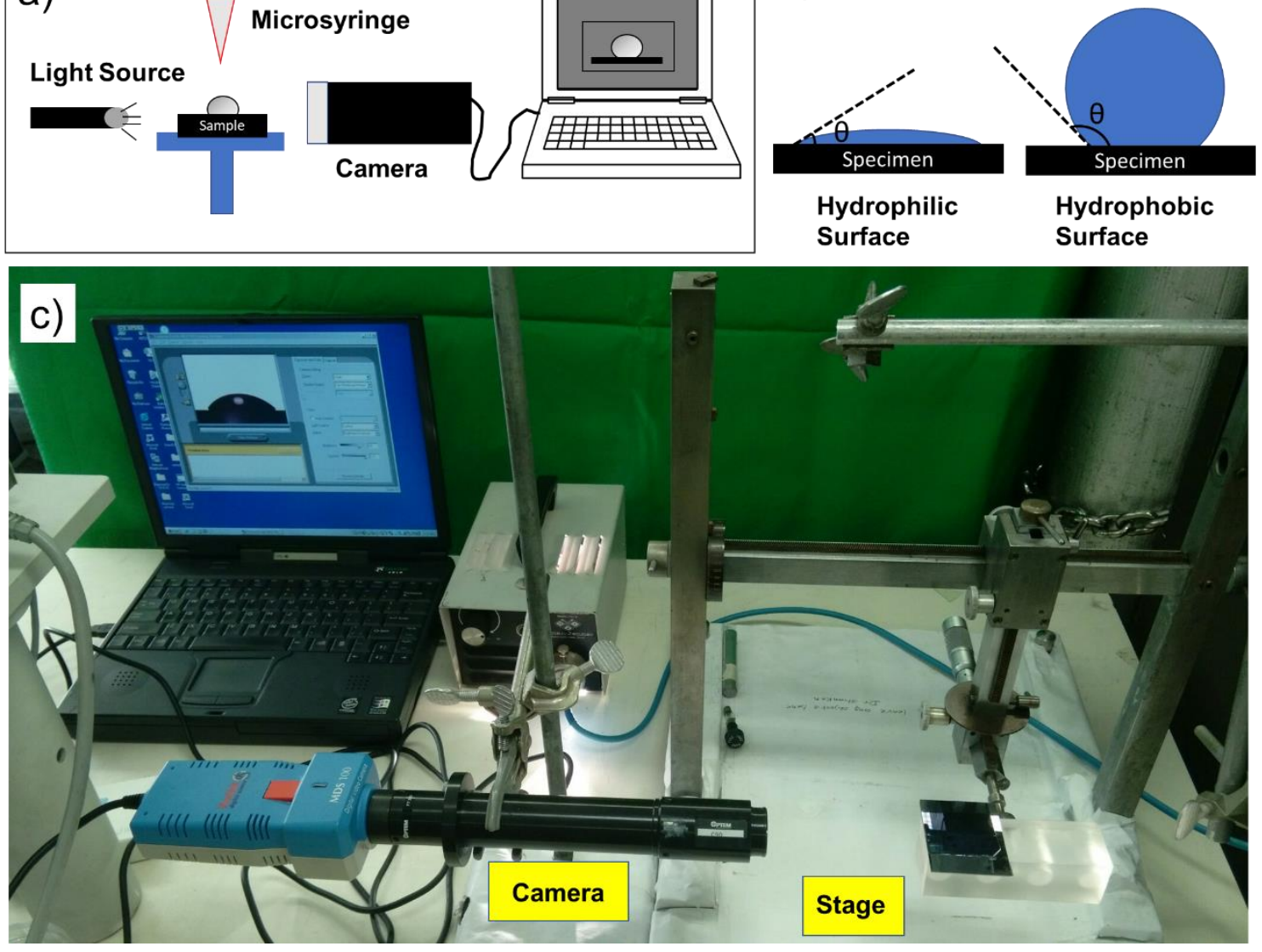

Figure 2.1. Schematic diagram depicting water contact angle measurement set up shown in a), and definition of the hydrophilic and hydrophobic surface shown in image b). Digital photograph in c), shows the laboratory fabricated CA measurement setup.

\subsubsection{Electromagnet system}

Unidirectional alignment of nickel coated copper nanowires $(\mathrm{Cu} / \mathrm{Ni} \mathrm{NWs})$, experiments were carried out by applying a uniform magnetic field in electromagnet system, see Figure 2.2 a \& b. GMW electromagnet system, Model-3470 with the dimensions of 45 and $30 \mathrm{~mm}$ of the pole diameter and pole gap respectively was used. Magnetic field strength can be varied by changing the current through magnet coil, Figure $2.2 \mathrm{c}$. 

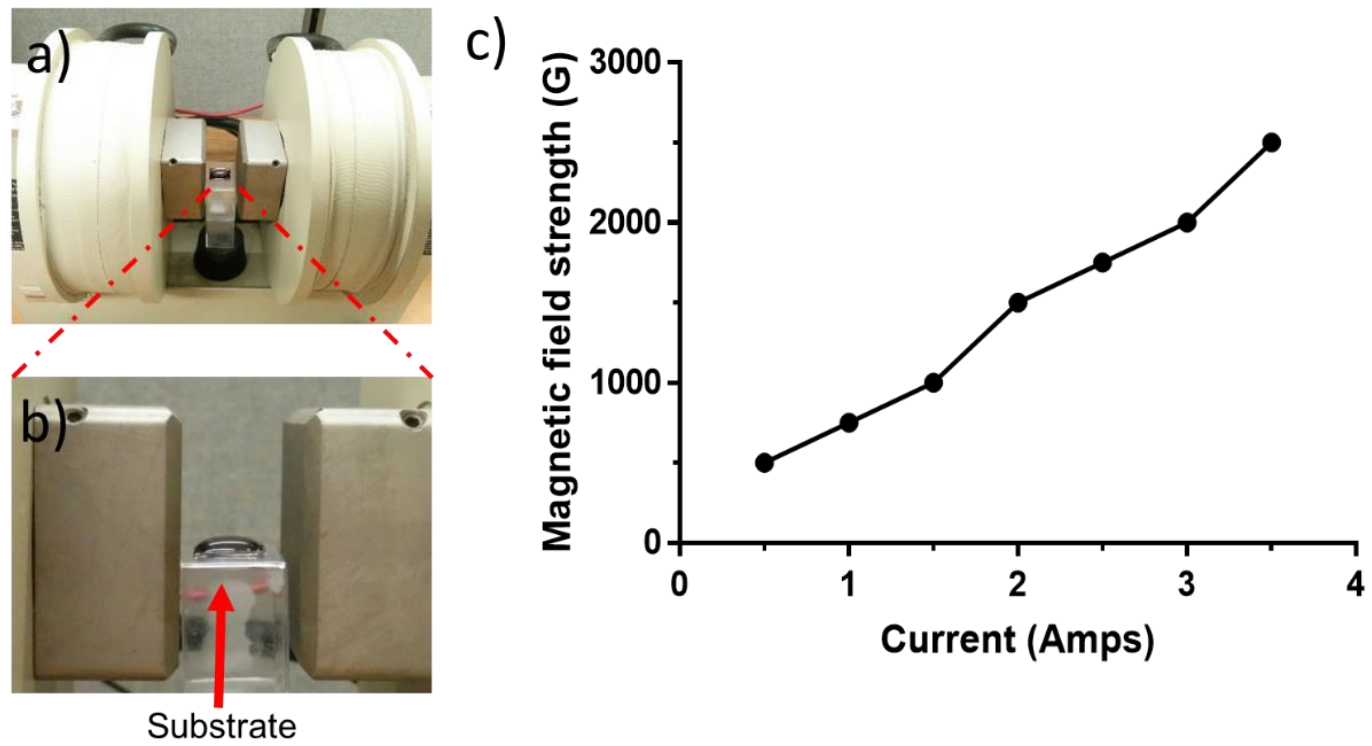

Figure 2.2. a) and b) showing digital photographs of electromagnet setup used for magnetic field assisted alignment of $\mathrm{Cu} / \mathrm{Ni}$ NWs. Plot c) showing change in magnetic field strength as a function of applied current.

\subsubsection{Scanning Electron Microscopy (SEM)}

Morphology and dimensions of nanomaterials were investigated by scanning electron microscopy (SEM). FEI Sirion XL30 SEM equipped with field emission electron gun which operates from $200 \mathrm{~V}$ to $30 \mathrm{kV}$ was used. Also, the microscope was equipped with Oxford energy-dispersive X-ray spectroscopy (EDS) and electron backscatter diffraction (EBSD) detectors. The EDS detector enabled the elemental composition and distribution analysis from photoelectrons generated from the specimen. Crystallographic orientation distribution was obtained from electron backscatter diffraction (EBSD) or transmission Kikuchi diffraction (TKD) patterning techniques as shown schematically in Figure 2.3. EBSD is a conventional technique which uses backscattered electrons, to study the orientation of ultrafine-grained metals and alloys, with grain sizes down to the 
submicron scale. However, the spatial resolution of the EBSD technique, even in the advanced SEM equipped with FEG electron sources, is limited to $20 \mathrm{~nm}$ for dense materials, and $50 \mathrm{~nm}$ for lighter materials such as $\mathrm{Mg}$ and $\mathrm{Al}^{63,64}$. Also, the resolution is inversely related to the sample tilt angle. Typically, EBSD sample holder is tilted to $70^{\circ}$ with respect to the horizontal plane, which results in the poor ( $\sim 3$ times worse) spatial resolution. Hence, conventional EBSD in an SEM, cannot be applied as a routine characterization tool for nanostructured/nano-sized materials.

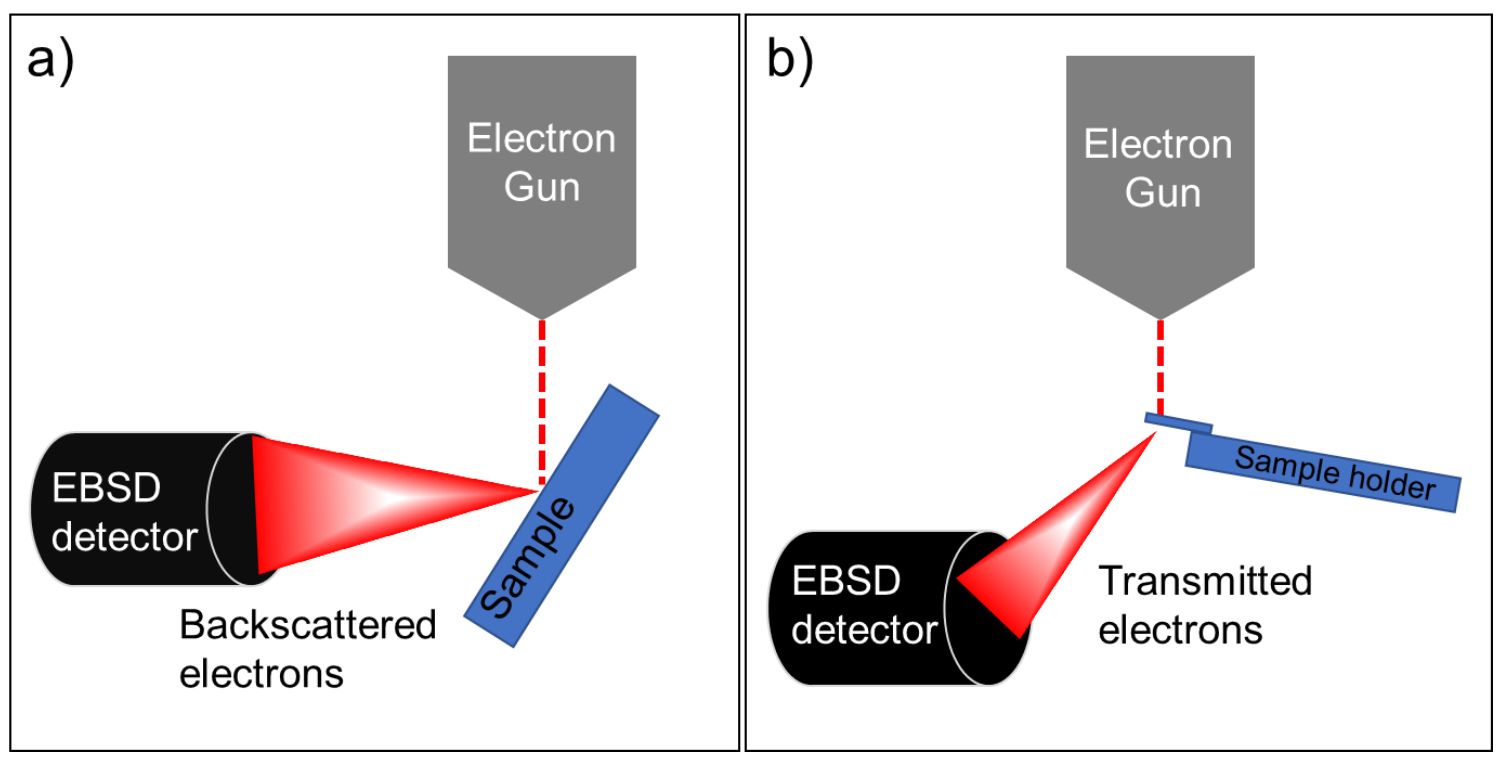

Figure 2.3. Schematic diagrams illustrating traditional EBSD a), and transmitted EBSD (t-EBSD or TKD) b), techniques used for crystallographic orientational mapping.

\section{Transmission Kikuchi Diffraction (TKD) pattern data collection:}

TKD is also known as transmitted electron backscatter diffraction (t-EBSD), and this offers significantly better spatial resolution than the conventional EBSD. TKD makes use of an EBSD detector and software application to capture and analyze the angular intensity 
variation of transmitted electrons. Transmitted electron diffraction patterns originate from very close to the bottom surface of the sample. TKD working principles and data collection procedures are discussed in detail elsewhere ${ }^{64,65}$.

The sample preparation for TKD studies involved drop casting of a dilute NWs solution (dispersed in ethanol) on a TEM grid. The TEM grid was mounted onto the custom-made sample holder (see Figure 2.4a). The TEM grid loaded sample holder was placed in the path of incident electron beam like the image shown in Figure 2.4b. Working distance, tilt angle and operating voltages had to be optimized to get high-resolution diffraction patterns.
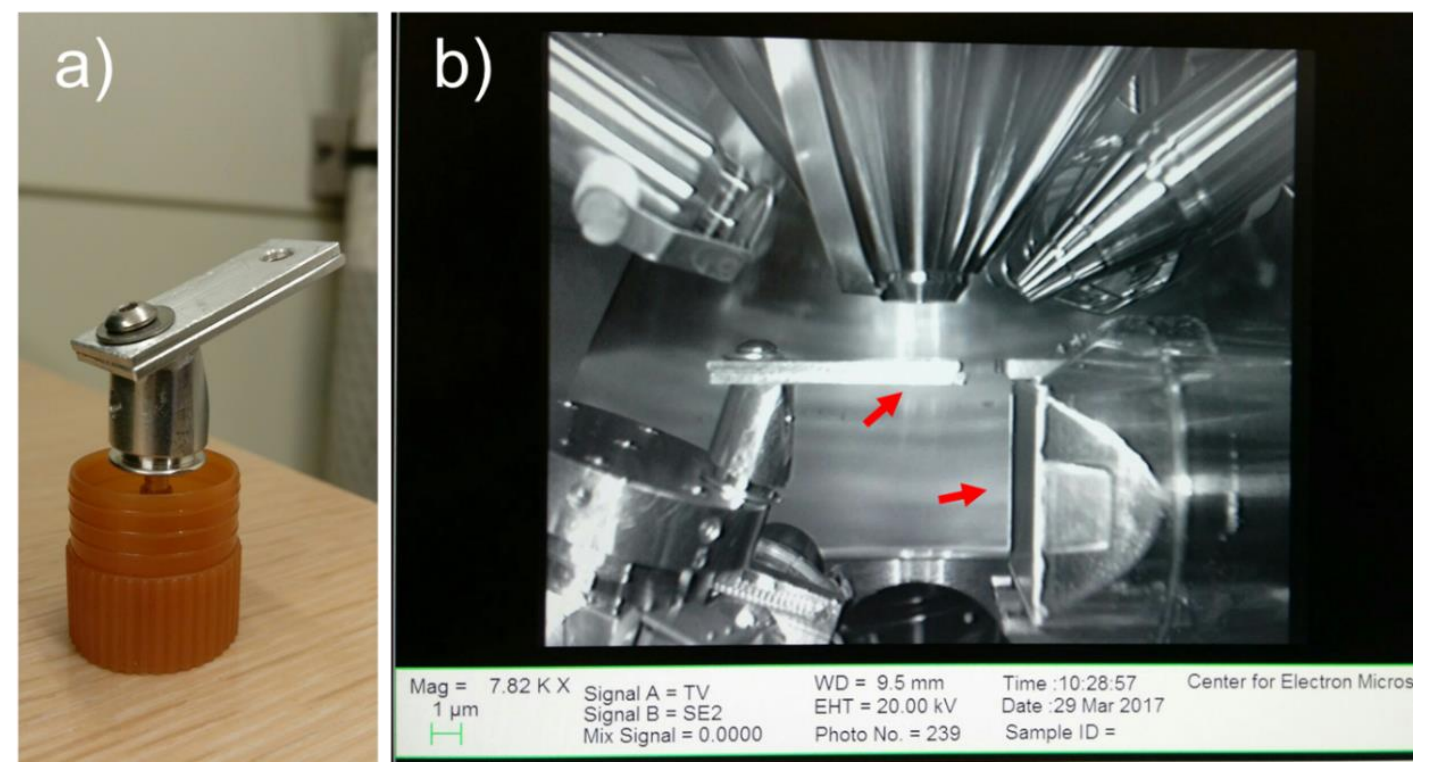

Figure 2.4. Digital photographs showing custom-made sample holder for t-EBSD data collection of $\mathrm{Cu}$ NWs a), and SEM chamber highlighting (pointed with red arrows) the positions of the sample holder and EBSD detector.

\subsubsection{Transmission Electron Microscopy (TEM)}


High-resolution images of $\mathrm{Cu}$ NWs were collected in FEI Tecnai F-20 TEM/STEM equipped with Gatan dark and bright field detectors for STEM and Gatan ultra-scan CCD ( $2 \mathrm{kx} 2 \mathrm{k})$ camera. Sample preparation involved drop casting of a $\mathrm{Cu}$ NWs dilute $(0.05$ $\mathrm{mg} / \mathrm{mL})$ ethanol solution $(20 \mu \mathrm{L})$ on the copper grid (200 mesh).

\subsubsection{Optical Microscopy}

Olympus BX51-P Optical microscopy was used to study the positioning and orientation of nanowires. It is equipped with a halogen lamp (100 Watts) and Canon high definition camera to capture the micrographs allowing a maximum lens magnification of $100 \mathrm{X}$.

\subsection{Materials and methods}

\subsubsection{Materials}

\section{Materials used for copper nanowires synthesis and nickel electroless coating:}

Copper nitrate $\left(\mathrm{Cu}\left(\mathrm{NO}_{3}\right)_{2} 2.5 \quad \mathrm{H}_{2} \mathrm{O}, \geq 99.99 \%\right.$, 19004-19-4), Ethylenediamine $\left(\mathrm{C}_{2} \mathrm{H}_{8} \mathrm{~N}_{2}, 99+\%\right.$, extra pure, 107-15-3), Hydrazine $\left(\mathrm{N}_{2} \mathrm{H}_{4}, 35\right.$ wt \% in $\left.\mathrm{H}_{2} \mathrm{O}, 302-01-2\right)$, Ethanol $\left(\mathrm{C}_{2} \mathrm{H}_{5} \mathrm{OH}, 99.99 \%, 64-17-5\right)$, Sodium hydroxide $(\mathrm{NaOH}, \geq 97 \%$, pellets, 1310-732), Ethylene Glycol $\left(\mathrm{C}_{2} \mathrm{H}_{6} \mathrm{O}_{2}, \geq 99.8 \%, 107-21-1\right)$, and Nickel Nitrate $\left(\mathrm{Ni}\left(\mathrm{NO}_{3}\right)_{2} 6 \mathrm{H}_{2} \mathrm{O}\right.$, 99.99\%, 13478-00-7) were used as received from Sigma Aldrich, without further purification.

\section{Materials used for surface functionalization of CDO wafer:}

The materials tert-Butyldimethyl silanol $\left(\left(\mathrm{CH}_{3}\right)_{3} \mathrm{CSi}\left(\mathrm{CH}_{3}\right)_{2} \mathrm{OH}\right.$, 99\%, 18173-643), tert-butyl dimethyl silyltrifluoro methanesulfonate $\left(\mathrm{CF}_{3} \mathrm{SO}_{3} \mathrm{Si}\left(\mathrm{CH}_{3}\right)_{2} \mathrm{C}\left(\mathrm{CH}_{3}\right)_{3}, 98 \%\right.$, 69739-34-0), trimethyl aluminum $\left(\mathrm{Al}\left(\mathrm{CH}_{3}\right)_{3}, 97 \%, 75-24-1\right)$, trifluoro methane sulfonic anhydride $\left(\left(\mathrm{CF}_{3} \mathrm{SO}_{2}\right)_{2} \mathrm{O}, \geq 99 \%, 358-23-6\right)$, ethoxytriphenylsilanol $\left(\mathrm{C}_{20} \mathrm{H}_{20} \mathrm{OSi}, \geq 99 \%\right.$, 
1516-80-9), chloroform $\left(\mathrm{CHCl}_{3}, \geq 99 \%, 67-66-3\right)$, anhydrous toluene $\left(\mathrm{C}_{6} \mathrm{H}_{5} \mathrm{CH}_{3}, 99.8 \%\right.$, 108-88-3) diisobutylaluminumhydride $\left(\left(\left(\mathrm{CH}_{3}\right)_{3} \mathrm{CHCH}_{2}\right)_{2} \mathrm{AlH}, 1191-15-7\right)$, 1-octadecene $\left(\mathrm{C}_{18} \mathrm{H}_{36}, \geq 95 \%, 112-88-9\right)$, octadecyltrichlorosilane $\left(\mathrm{C}_{18} \mathrm{H}_{38} \mathrm{SiCl}_{3}, \geq 90 \%, 112-04-9\right)$ and 2propene-1-thiol $\left(\mathrm{CH}_{2}=\mathrm{CHCH}_{2} \mathrm{SH}, \sim 60 \%, 870-23-5\right)$ were all reagent grade, purchased from Sigma-Aldrich and used as received. Sigma-Aldrich was the source of amorphous silica gel (high-purity grade (Davisil Grade 62), $\mathrm{SiO}_{2}, 60-200$ mesh, Chromatography grade, Fisher Scientific, 7631-86-9). Intel Corporation provided $\mathrm{CDO}$ and $\mathrm{SiO}_{\mathrm{x}}$ coated silicon wafers.

\section{Materials used for Photolithography patterning:}

MEGAPOSIT TM SPR 220- 3.0 photoresist (Shipley), Sodium hydroxide $(\mathrm{NaOH}, \geq 97 \%$, pellets, 1310-73-2, Sigma Aldrich) and acetone $\left(\mathrm{C}_{3} \mathrm{H}_{6} \mathrm{O}, \geq 99 \%, 67-64-1\right.$, Sigma Aldrich).

\subsubsection{Copper nanowires synthesis method}

Copper nanowires $(\mathrm{Cu} \mathrm{NWs}$ ) were synthesized in a solution based approach using ethylenediamine (EDA) as a growth directing agent and hydrazine as a reducing agent in an aqueous $\mathrm{NaOH}$ solution ${ }^{33,58}$. This work explored the effect of reducing agent concentration and synthesis temperature on dimensions and morphology of nanowires and established the optimized concentration of reagents for synthesizing surface morphology controlled, high-aspect-ratio (Length/diameter) $\mathrm{Cu}$ NWs. More synthesis details appear in Chapter-3.

\subsubsection{Electroless coating of nickel on $\mathrm{Cu}$ NWs surface}

Copper nanowires were coated with a thin layer $(\sim 20 \mathrm{~nm})$ of nickel $(\mathrm{Ni})$ in an electroless coating method. The nickel electroless coating procedure involves preparation 
of a well-dispersed solution of $\mathrm{Cu} \mathrm{NWs}$, nickel nitrate $\left(\mathrm{Ni}\left(\mathrm{NO}_{3}\right)_{2}\right)$ and reducing agent hydrazine in an ethylene glycol solution ${ }^{59}$. After heating the as-prepared solution in the oil bath at $120{ }^{\circ} \mathrm{C}$ for 10 minutes produced floating of nickel coated $\mathrm{Cu}$ NWs at the air-liquid interface.

\subsubsection{Unidirectional alignment of $\mathrm{Cu} / \mathrm{Ni} \mathrm{NWs}$}

Unidirectional alignment or self-assembly of NWs into interconnect channels was carried out by placing a plane or lithography patterned substrate in between the electromagnet poles presented in digital photographs shown in Figure $2.5 \mathrm{a} \& \mathrm{~b}$. The $\mathrm{Cu} / \mathrm{Ni}$ NWs aqueous suspension was deposited on top the substrate and allowed to evaporate under ambient conditions in the presence of magnetic field. Figure $2.5 \mathrm{c} \& \mathrm{~d}$ depict the schematic of $\mathrm{Cu} / \mathrm{Ni} \mathrm{NWs}$ arrangement after the solvent was allowed to dry in the absence and the presence of magnetic field respectively. In the absence of magnetic field, all the NWs oriented in random directions. Whereas in a high magnetic field, all the NWs oriented in the applied magnetic field direction. 


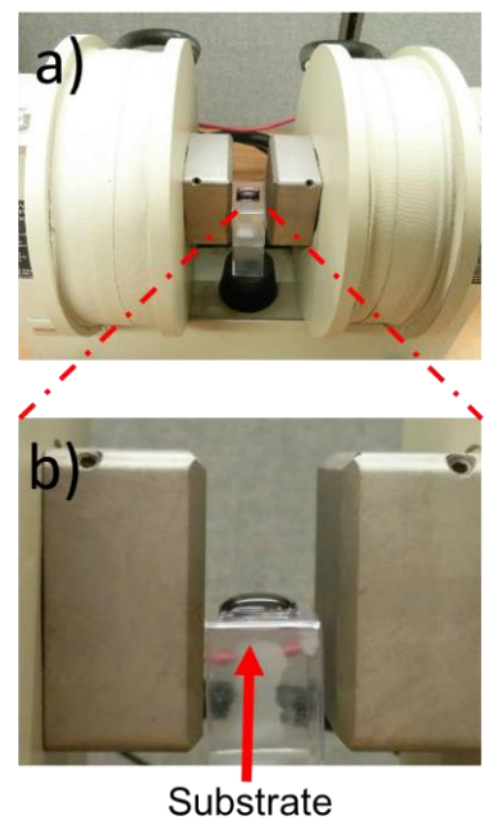

c)

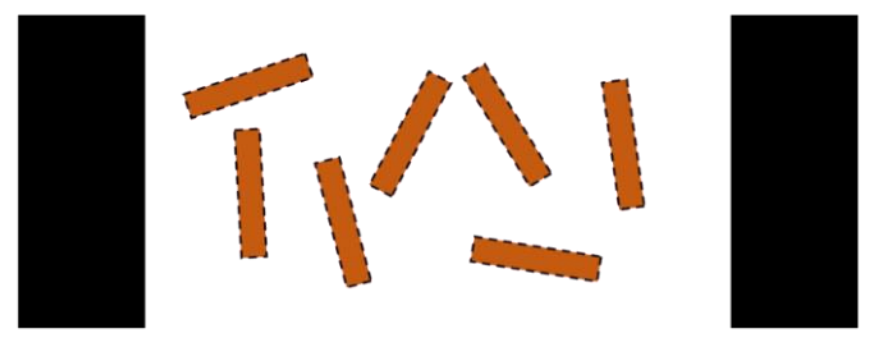

d)
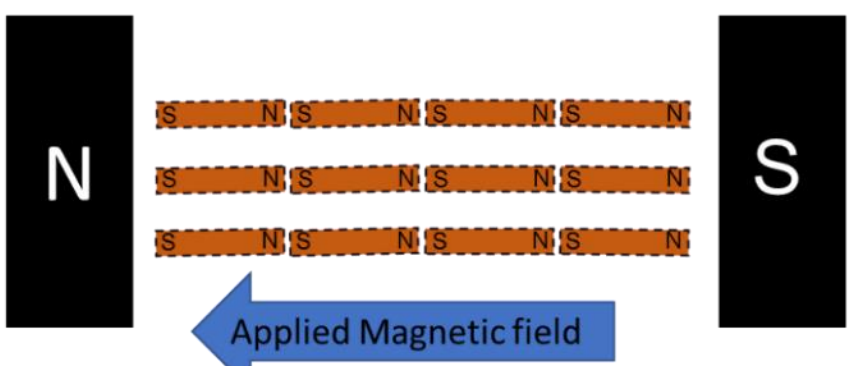

Figure 2.5. Digital photographs of GMW electromagnet setup in top view a) and side view b). Schematic is shown in image c) and d) depicts an arrangement of $\mathrm{Cu} / \mathrm{Ni}$ NWs in absence and presence of magnetic field.

Also, the uniform alignment of NWs on a given substrate requires a uniform magnetic field. Therefore, for all the NWs alignment experiments, substrates were placed in between the poles of the electromagnets. In comparison to the diameter of electromagnet poles, the substrates used were two-fold smaller in dimension.

\subsubsection{Common synthetic procedure for selective functionalization of Si-OH moieties}

The following common protocol was used to selectively functionalize the Si-OH functional groups on organic molecules (t-Butyldimethylsilanol and Triphenylsilanol), silica gel, $\mathrm{SiO}_{\mathrm{x}}$ and CDO wafer substrates. 


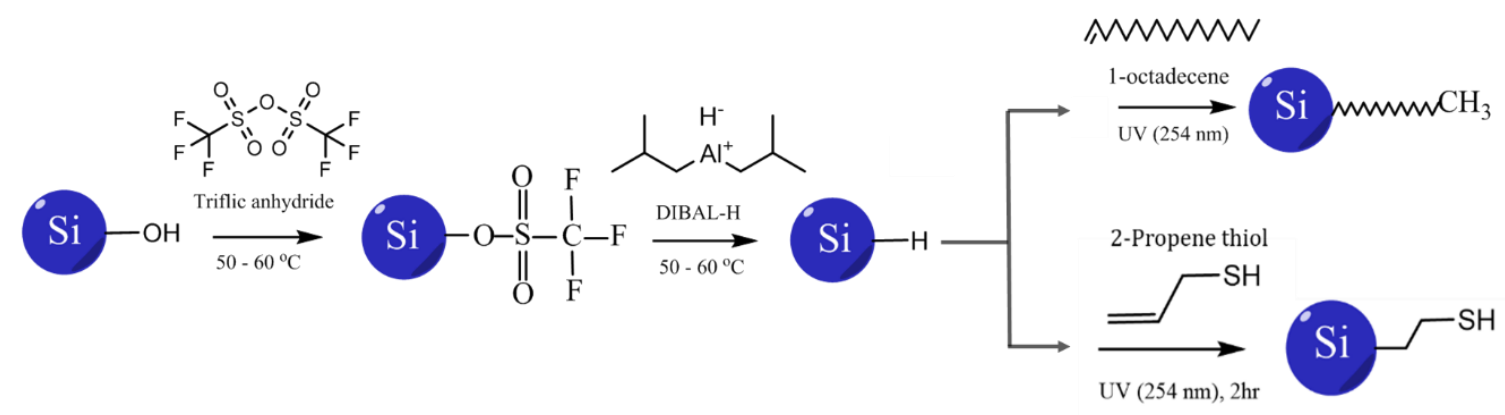

Scheme 2.1. Schematic showing common reaction pathway for selective functionalization of Si$\mathrm{OH}$ functional groups to Octadecyl and thiol-derivatized silicon.

The first step in the reaction scheme employed $0.1 \mathrm{M}$ triflic anhydride $\left(\left(\mathrm{CF}_{3} \mathrm{SO}_{2}\right)_{2} \mathrm{O}\right)$ in anhydrous toluene at $50-60{ }^{\circ} \mathrm{C}$ for 48 hours to esterify the $\mathrm{Si}-\mathrm{OH}$ functional group to yield silyltrifluoromethane sulfonate ( $\mathrm{Si}-\mathrm{O}-\mathrm{Tf}$, where $\mathrm{Tf}$ indicates the $\mathrm{SO}_{2} \mathrm{CF}_{3}$ group). In the second step, 0.1 M DIBAL-H (di-isobutylaluminum hydride, $\left.(\mathrm{CH} 3)_{2} \mathrm{CHCH}_{2}\right)_{2} \mathrm{AlH}$ ) reagent at $50-60{ }^{\circ} \mathrm{C}$ for 48 hours reduced the triflate intermediate $\left(\mathrm{Si}-\mathrm{O}-\left(\mathrm{SO}_{2}\right) \mathrm{CF}_{3}\right)$ to silicon hydride. Because hydrogen-terminated silicon is unstable, $\mathrm{Si}-\mathrm{H}$ was further treated with 1-octadecene $\left(\mathrm{CH}_{3}\left(\mathrm{CH}_{2}\right){ }_{15} \mathrm{CH}=\mathrm{CH}_{2}\right)$ in the presence of $\mathrm{UV}$ light $(254 \mathrm{~nm})$ for 2 hours to produce $\mathrm{Si}-\mathrm{R}\left(\mathrm{Si}-\mathrm{C}_{18} \mathrm{H}_{37}\right)$. For thiol (-SH) functionalization, hydrogen-terminated silicon was treated with 2-propene thiol $\left(\mathrm{CH}_{2}=\mathrm{CH}-\mathrm{CH}_{2}-\mathrm{SH}\right)$ under similar conditions. All reactions were carried out in dry $\mathrm{N}_{2}$ within a glovebox. Between each set of reactions, silica gel, $\mathrm{SiO}_{\mathrm{x}}$ wafer, and $\mathrm{CDO}$ wafers were thoroughly washed with anhydrous toluene.

\subsection{6. $\mathrm{RCA}$ cleaning procedure for $\mathrm{SiO}_{\mathrm{x}}$ and $\mathrm{CDO}$ wafers}

$\mathrm{SiO}_{\mathrm{x}}$ and $\mathrm{CDO}$ coupons were cleaned with a standard RCA-1 solution. The cleaning protocol involved immersion of $1 \times 2 \mathrm{~cm}$ coupons into RCA-1 solution (a mixture of 1:1:5 
volume ratios of $\mathrm{NH}_{4} \mathrm{OH}: \mathrm{H}_{2} \mathrm{O}_{2}$ : DI water) at $70{ }^{\circ} \mathrm{C}$ for 10 minutes. RCA-1 solution is a strong oxidizer. It will effectively remove organic contamination from the wafer surface. After RCA cleaning, wafers were thoroughly washed in DI water, chloroform and, then anhydrous toluene solvent before proceeding to the functionalization reaction.

\subsubsection{Photolithography}

Photolithography or UV lithography refers to the process of using UV light to transfer the geometric pattern from a photomask to the thin film of photoresist. The schematic diagram shown in Figure 2.6, outlines the major steps in the photolithography process.

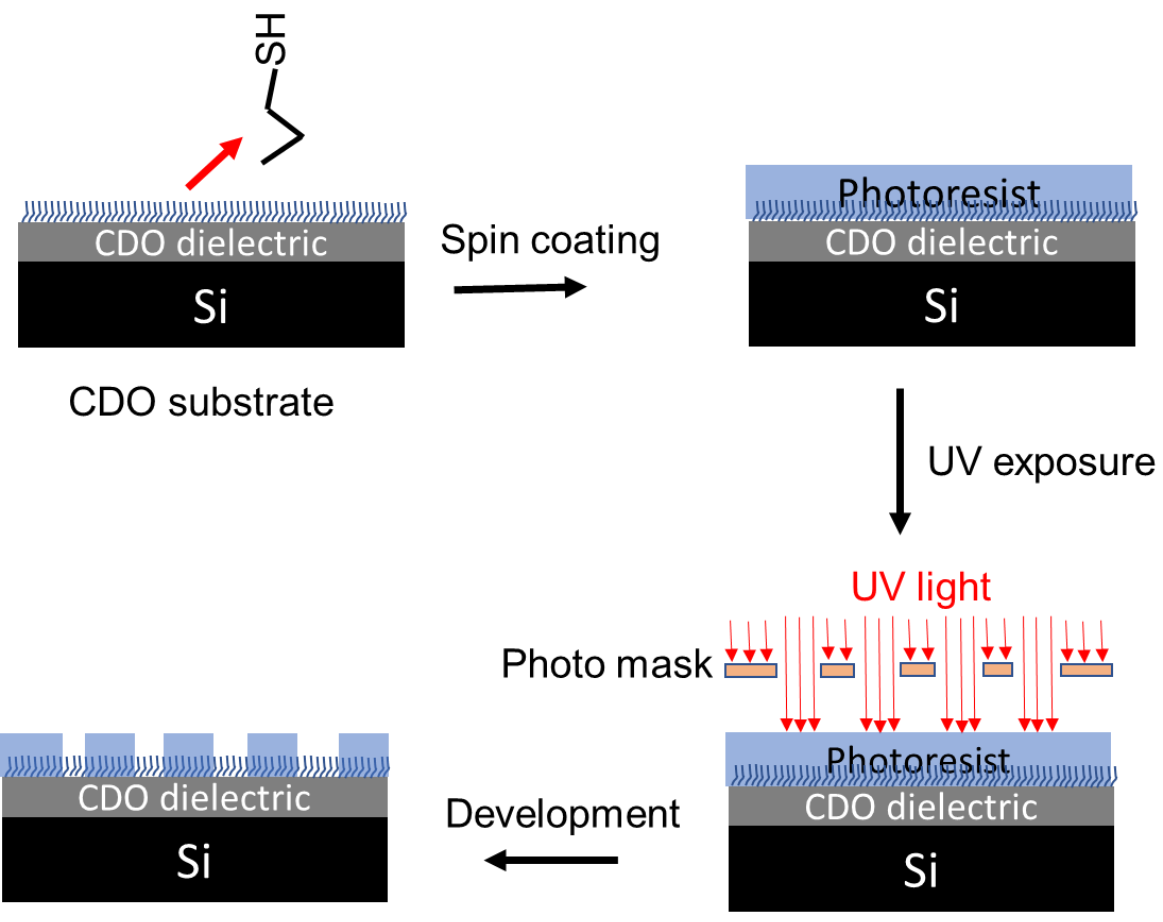

Figure 2.6. Schematic diagram showing photolithography process flow.

The procedure involved, spin coating of a thin film of positive tone photoresist (SPR 220 3.0, Shipley) on to the thiol-functionalized CDO substrate. The thickness of the as- 
coated photoresist film was measured to be $3.0 \pm 0.2 \mu \mathrm{m}$. The as-coated wafer was prebaked for 90 secs at $110{ }^{\circ} \mathrm{C}$, which removed volatile organic solvents from the photoresist film. The circuit pattern ( $4 \mathrm{~mm}$ long, $300 \mu \mathrm{m}$ wide channel was used for self-assembly of NWs) was transferred into the photoresist film by exposing the photoresist coated wafer to UV light $(365 \mathrm{~nm})$ through a photomask in a benchtop projection mask aligner (Tamarack scientific Co., Model-162), see Figure 2.7. After UV exposure, the substrate was postbaked at $110{ }^{\circ} \mathrm{C}$, to improve the solubility of UV exposed the photoresist. Finally, the postbaked substrate was developed in a dilute alkaline medium, which dissolved the UV exposed photoresist leaving the unexposed regions on the CDO substrate. The exact process conditions of the Photolithography patterning are summarized in table 2.1 below.

\begin{tabular}{|l|l|}
\hline Photolithography processing steps & \multicolumn{1}{c|}{ Conditions } \\
\hline Photoresist & SPR 2203.0 (Shipley), Positive tone resist \\
\hline Spin coating speed & $3500 \mathrm{RPM}$ for 4 minutes \\
\hline Pre-exposure bake & $110^{\circ} \mathrm{C}$ for $90 \mathrm{Sec}$ \\
\hline UV exposure duration & 5 minutes \\
\hline Post-exposure bake & $110^{\circ} \mathrm{C}$ for $90 \mathrm{Sec}$ \\
\hline Developer solution & $0.1 \mathrm{M} \mathrm{NaOH}$ aqueous solution \\
\hline Development time & $40 \mathrm{Sec}$ \\
\hline
\end{tabular}

Table 2.1. Photolithography process steps and conditions are presented here. 


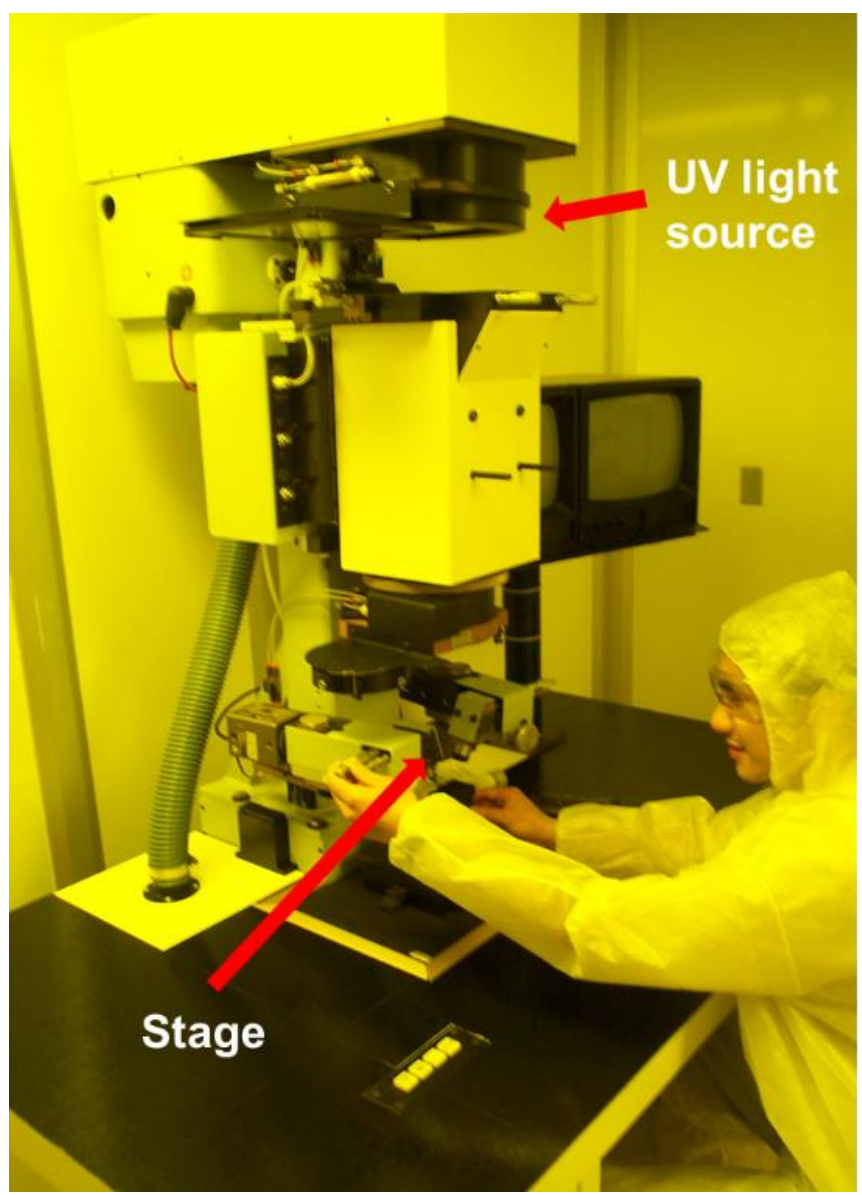

Figure 2.7. Digital photograph showing projection mask UV aligner (Tamarack scientific Co., Model-162) used for photolithography patterning (capable of patterning resolvable feature size of $1 \mu \mathrm{m})$.

\subsubsection{Electrical characterization of self-assembled interconnect channels}

The self-assembly of nanowires into photolithography patterned channel protocol is discussed in detail in Chapter-5. Electrical characterization of the as assembled NWs channel is carried out by fabricating the silver contact pads (High purity silver paint, SPI supplies, CAS \# 05002-AB, lot no \# 1160331) on both ends of the channel and measured the resistance values using Keithley programmable multimeter (Model-617). 


\section{CHAPTER THREE}

\section{COPPER NANOWIRES SYNTHESIS AND MAGNETIC FIELD ASSISTED}

\section{ALIGNMENT}

In recent years, the synthesis of one dimensional (1D) nanostructures such as metallic NWs (Ag NWs, Cu NWs, Au NWs, and Pt NWs) or carbon nanotubes (CNT) and their assembly into functional devices have received special interest owing to its size and structuredependent unique properties. Among all metallic nanowires, copper nanowires (Cu NWs) have attracted considerable attention lately due to their excellent electrical conductivity (only 7\% less conductive than silver), thermal, mechanical properties, and cheap precursor cost (100 times cheaper than silver). In literature, various Cu NWs synthesis methods were discussed such as chemical vapor deposition (CVD) ${ }^{53}$, porous templates ${ }^{26,54}$, hydrothermal/solvothermal ${ }^{57}$, and solution-based approaches ${ }^{33,35,58}$. However, in all the above methods, the ability to precisely control the morphology and dimensions of NWs was not possible. This thesis work adopted a solution based synthesis approach developed by Zeng et al., ${ }^{58}$ and Wiley et al., ${ }^{33,66}$. This thesis studied the effects of reagent concentrations and synthesis temperature on morphology and aspect ratio controlled $\mathrm{Cu}$ NWs synthesis.

The bottom-up or hybrid approaches of NWs assembly into nanodevices requires simple, scalable and robust techniques to precisely control the orientation and positioning of NWs. For Cu NWs, very limited success has been achieved in controlling the orientation via electrochemical deposition of copper into a porous template ${ }^{26,54}$, the thermal assisted growth of freestanding NWs on a copper substrate ${ }^{67}$ and vertically aligned NWs in a CVD 
technique ${ }^{53}$. The challenges in these techniques include high process complexity, poor yield, and the as-grown NWs stuck to the substrate (hard to manipulate the location); so $\mathrm{Cu}$ NWs are very challenging to transfer onto another desired substrate. To date, a simple and robust technique to control the orientation of $\mathrm{Cu}$ NWs has not been demonstrated. Here, for the first time, present work illustrates the magnetic field assisted unidirectional alignment of Cu NWs by coating them with a thin layer $(\sim 20 \mathrm{~nm})$ of ferromagnetic nickel (Ni). Upon applying of the magnetic field, $\mathrm{Cu} / \mathrm{Ni} \mathrm{NWs}$ in the solution phase align in the applied magnetic field direction and as the solvent dries aligned NWs deposit on the substrate. In the subsequent sections, copper nanowires synthesis, an electroless coating of nickel onto $\mathrm{Cu}$ NWs surface (Cu/Ni NWs) and magnetic field assisted alignment of $\mathrm{Cu} / \mathrm{Ni}$ NWs have been discussed in detail.

\subsection{Copper nanowires synthesis}

Copper nanowires were synthesized in a solution based approach, using copper nitrate $\left(\mathrm{Cu}\left(\mathrm{NO}_{3}\right)_{2} 2.5 \mathrm{H}_{2} \mathrm{O}\right)$ as a precursor, ethylenediamine (EDA, $\left.\mathrm{C}_{2} \mathrm{H}_{4}\left(\mathrm{NH}_{2}\right)_{2}\right)$ as a growth directing agent and hydrazine $\left(\mathrm{NH}_{2}-\mathrm{NH}_{2}\right)$ as a reducing agent. The synthesis approach followed the method published in the literature ${ }^{33,58,68,69}$.

3.1.1. Cu NWs synthesis procedure: In brief, the procedure involved the mixing of a copper nitrate solution $(0.1 \mathrm{M}$ in DI water, $2 \mathrm{~mL})$ to a freshly prepared aqueous $\mathrm{NaOH}(15$ $\mathrm{M}, 40 \mathrm{~mL})$ solution. Ethylenediamine $(0.1 \mathrm{M}, 266 \mu \mathrm{L})$ and hydrazine $\left(9.66 \times 10^{-3} \mathrm{M}(35 \mu \mathrm{L})\right.$ to $\left.4.09 \times 10^{-3} \mathrm{M}(15 \mu \mathrm{L})\right)$ reagents were added followed by a thorough mixing for 3 and 2 min respectively, after adding each reagent. Upon addition of hydrazine, the solution turned clear suggesting the reduction of $\mathrm{Cu}^{+2}$ ions into $\mathrm{Cu}^{+1}$, After 30 minutes, a reddish-brown 
cake formed at the air-water interface leaving the clear solution at the bottom, see Figure 3.1c. NWs were collected and washed several times with DI water and ethanol in a centrifugation process, and finally stored in absolute ethanol solvent in a sealed glass vial.
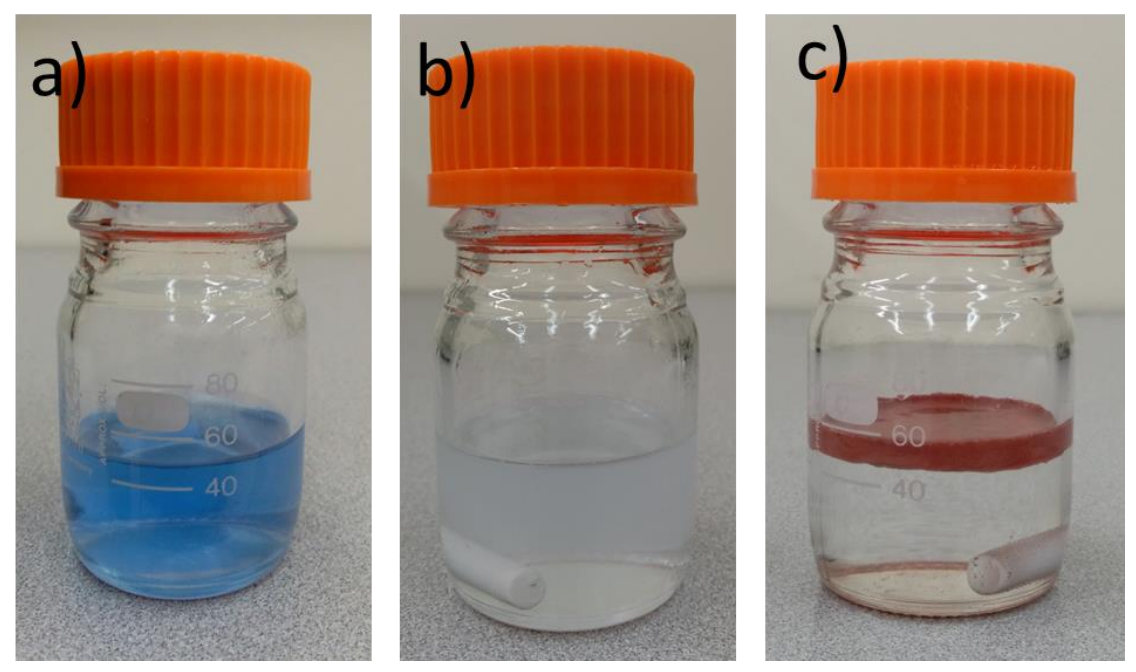

Figure 3.1. Digital images of $\mathrm{Cu}$ NWs reaction solution before adding hydrazine (a) and after adding hydrazine (b). After incubation for 30 minutes in hot water bath reddish-brown Cu NWs cake floating at the air-water interface.

The chemical reactions involved in $\mathrm{Cu}$ NWs synthesis are shown below ${ }^{33,35,58}$. Cupric $\left(\mathrm{Cu}^{2+}\right)$ ions react with hydroxyl $\left(\mathrm{OH}^{-}\right)$ions and form cupric hydroxide complex ions (see eq-1). The reducing agent, hydrazine, supplies electrons that are required for the reduction of cupric ions to copper $(\mathrm{Cu}(0))$. In the first step of the reduction process, cupric hydroxide $\left(\mathrm{Cu}^{2+}\right)$ complex ions are reduced to cuprous $\left(\mathrm{Cu}^{+}\right)$hydroxyl complex ions, (see eq-3) (solution color turns from blue to clear, See Figure $3.1 \mathrm{a}, \mathrm{b}$ ). And, in the second step, as cuprous ion complexes $\left(\mathrm{Cu}^{+}\right)$are reduced to metallic copper $\left(\mathrm{Cu}^{0}\right)$, (see eq-4). The capping agent ethylenediamine (EDA), directs the copper atoms into unidirectional growth to form nanowire morphology. 
$\mathrm{Cu}^{2+}+4 \mathrm{OH}^{-} \rightarrow \mathrm{Cu}(\mathrm{OH})_{4}^{2-}$ (blue solution)

$\mathrm{N}_{2} \mathrm{H}_{4}+4 \mathrm{OH}^{-} \rightarrow \mathrm{N}_{2}(\mathrm{~g})+4 \mathrm{H}_{2} \mathrm{O}+4 \mathrm{e}^{-}$

$2 \mathrm{Cu}(\mathrm{OH})_{4}{ }^{2-}+2 \mathrm{e}^{-} \rightarrow 2 \mathrm{Cu}(\mathrm{OH})_{2}^{-}($clear solution $)$

$2 \mathrm{Cu}(\mathrm{OH})_{2}^{-}+2 \mathrm{e}^{-} \rightarrow 2 \mathrm{Cu}(0)+2 \mathrm{OH}^{-}$

The amine functional groups in EDA capping agent act as a ligand to form the coordinate covalent bond and interact with unsaturated surface atoms in the nanocrystals ${ }^{70}$. The binding affinities of EDA are determined by the distinct atom geometries in various crystal planes. The capping agents on the nanocrystal act as a physical barrier to restrict the access of reagents in the capped facets and allowing the active growth process to occur in the uncapped regions ${ }^{70}$.

Copper nanowire growth mechanism: The growth mechanism of copper nanowire in an EDA and hydrazine assisted method have been discussed in detail by Wiley et al., ${ }^{35,71}$ In brief, the mechanism involves during the initial stages of reduction process $\mathrm{Cu}(\mathrm{OH})_{4}{ }^{2-}$ ions are reduced to $\mathrm{Cu}(\mathrm{OH})_{2}{ }^{-}$ions and $\mathrm{Cu}_{2} \mathrm{O}$ nanoparticles (the resultant solution appears as translucent, see Figure 3.1b). The capping agent EDA helps in preventing the precipitation of these $\mathrm{Cu}_{2} \mathrm{O}$ nanoparticles by capping the surface. And, keeping these nanoparticles suspended in the solution are very crucial for the initiation of nanowire growth process. The EDA coated $\mathrm{Cu}_{2} \mathrm{O}$ nanoparticles are further reduced by hydrazine to form irregular shaped polycrystalline metallic copper aggregates with the size ranging from 200 to 500 $\mathrm{nm}$ in diameter ${ }^{70}$. The surface of these copper aggregates consists some of the pentagonal twinned secondary particles $(\sim 40 \mathrm{~nm})$ which will serve as a seed to sprout nanowire ${ }^{71}$. And, 
during the nanowire growth process, EDA preferentially caps the higher surface energy $\{100\}$ crystal facets and leaving the lower energy $\{111\}$ crystal facets (nanowire tip). The $\mathrm{Cu}(\mathrm{OH})_{2}{ }^{-}$ions are reduced to metallic copper $(\mathrm{Cu}(0))$ by hydrazine at nanowire tip which possesses $\{111\}$ crystal facet and promotes the anisotropic growth of nanowire morphology $\mathrm{y}^{35}$

In this synthesis, NWs dimensions such as aspect ratio (length/diameter) and surface morphology depended critically on the reagent concentrations and synthesis conditions. Here, systematic studies of the effect of synthesis temperature and concentration of reducing agent on NWs dimensions and morphology were investigated.

\subsubsection{Effect of synthesis temperature}

The effect of synthesis temperature was studied at $60,70,80$ and $90{ }^{\circ} \mathrm{C}$ respectively. Except for the temperature, all other reaction parameters were kept constant. Figure 3.2 shows SEM images of $\mathrm{Cu}$ NWs synthesized at various temperatures, which indicates the decrease in aspect ratio as the synthesis temperature increase. Image-J software analyzed aspect ratios of $\mathrm{Cu}$ NWs synthesized at various temperatures are shown in Table 3.1. As the synthesis temperature increased to more than $90{ }^{\circ} \mathrm{C}$, all the copper atoms agglomerated and formed micrometer-sized (200 $\mathrm{nm}$ to $3 \mu \mathrm{m})$ sized copper aggregates. It was suggested that the formation $\mathrm{Cu}-\mathrm{NWs}$ is controlled by EDA which caps the side-wall surface of NWs. The potential reason for the formation of micron size aggregates at a higher temperature could be due to desorption of EDA from the NW surface, coupled with lower supersaturation of $\mathrm{Cu}$ atoms at temperatures above $90{ }^{\circ} \mathrm{C}$. 

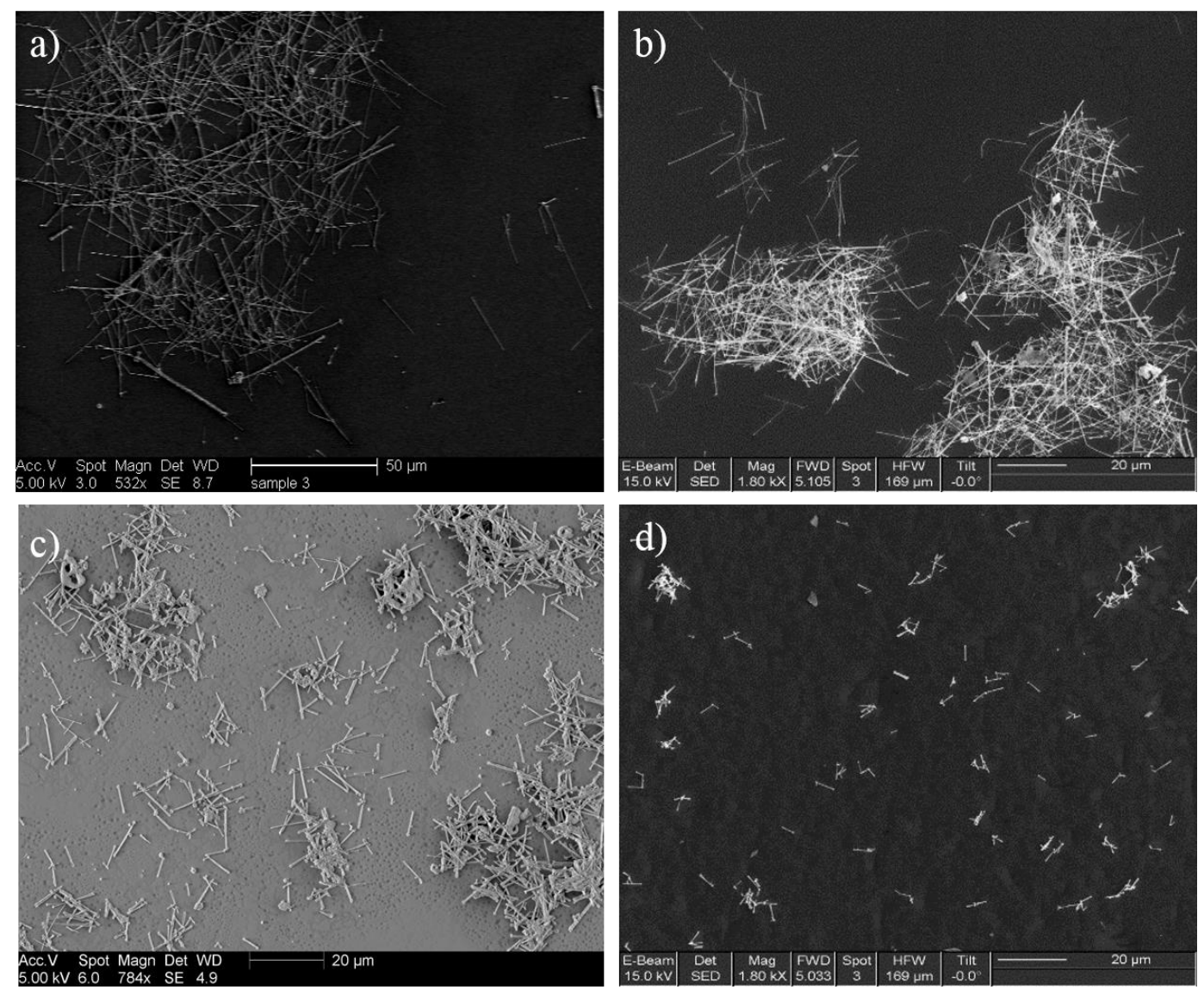

Figure 3.2. SEM images of copper nanowires synthesized at $60{ }^{\circ} \mathrm{C}(\mathrm{a}), 70{ }^{\circ} \mathrm{C}(\mathrm{b}), 80{ }^{\circ} \mathrm{C}(\mathrm{c})$, and $90{ }^{\circ} \mathrm{C}(\mathrm{d})$ respectively.

\begin{tabular}{cccc}
\hline $\begin{array}{c}\text { Temp. } \\
\left({ }^{\circ} \mathrm{C}\right)\end{array}$ & $\begin{array}{c}\text { Average } \\
\text { Length } \\
(\mu \mathrm{m})\end{array}$ & $\begin{array}{c}\text { Average } \\
\text { Diameter } \\
(\mathrm{nm})\end{array}$ & Aspect ratio \\
\hline 60 & $32 \pm 7$ & $160 \pm 30$ & 200 \\
70 & $25 \pm 6$ & $170 \pm 50$ & 147 \\
75 & $15 \pm 4$ & $210 \pm 60$ & 71 \\
80 & $7 \pm 2$ & $220 \pm 30$ & 32 \\
\hline
\end{tabular}

Table 3.1. Average lengths, diameters and aspect ratios of copper nanowires synthesized at 60, 70, 80 , and $90{ }^{\circ} \mathrm{C}$ respectively. 


\subsubsection{Effect of reducing agent concentration}

Nanowire anisotropic growth and thickening mechanisms were discussed by Wiley et al., and highlight the importance of reducing agent (hydrazine) concentration ${ }^{33,35,68}$. Similarly, the present investigation focused on the effect of hydrazine concentration on surface morphology and the aspect ratio of Cu NWs.
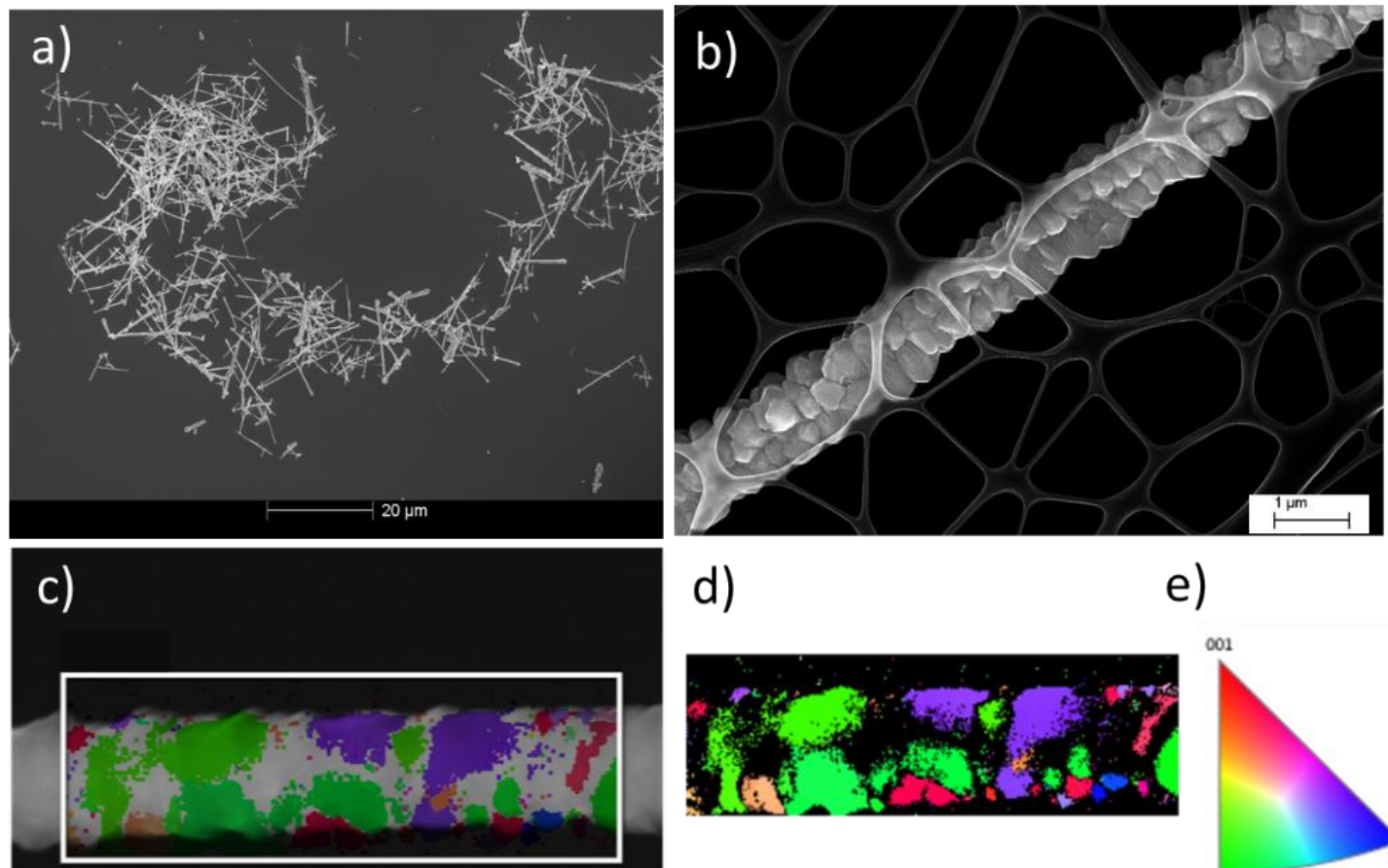

d)

e)
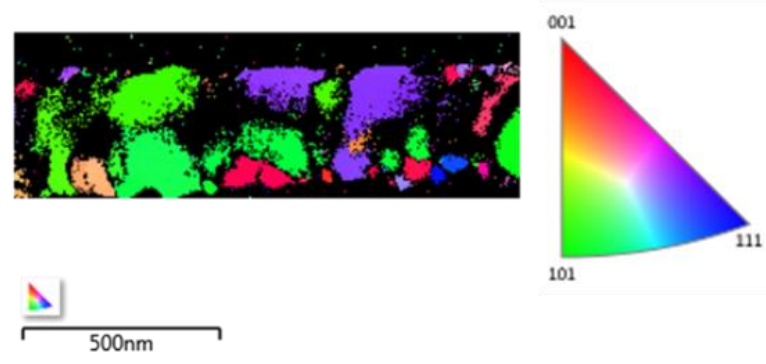

Figure 3.3. SEM images of $\mathrm{Cu}$ NWs synthesized using high concentration ( $35 \mu \mathrm{L})$ of reducing agent, Images shown at low a) and high b) magnification scales. TKD overlaid SEM image shown in c), and out-of-the-page (Z) direction crystallographic orientation is shown in d), highlight the thick and highly particle decorated NWs. e) crystal plane to color mapping scale. 
In these studies, except for hydrazine, all other reagent concentrations and conditions were maintained constant. See experimental section (3.1.1.) for more information about the synthesis procedure. Figure 3.3a \& b shows SEM images of $\mathrm{Cu}$ NWs synthesized using a high concentration $\left(9.66 \times 10^{-3} \mathrm{M}, 35 \mu \mathrm{L}\right)$ of reducing agent hydrazine. These images revealed that as-synthesized NWs featured low aspect ratio (L/D 20) with rough surface morphology suggestive of $\mathrm{Cu}$ nanoparticle decoration on the NW surface. To characterize the nanoparticle decoration, this study examined the crystallographic orientational mapping of these particulates on the NW surface using SEM. Transmitted electron backscattered diffraction (t-EBSD) orientational mapping images shown in Figure 3.3 c \& d displayed various crystallographic orientations of these (adsorbed) nanoparticles.
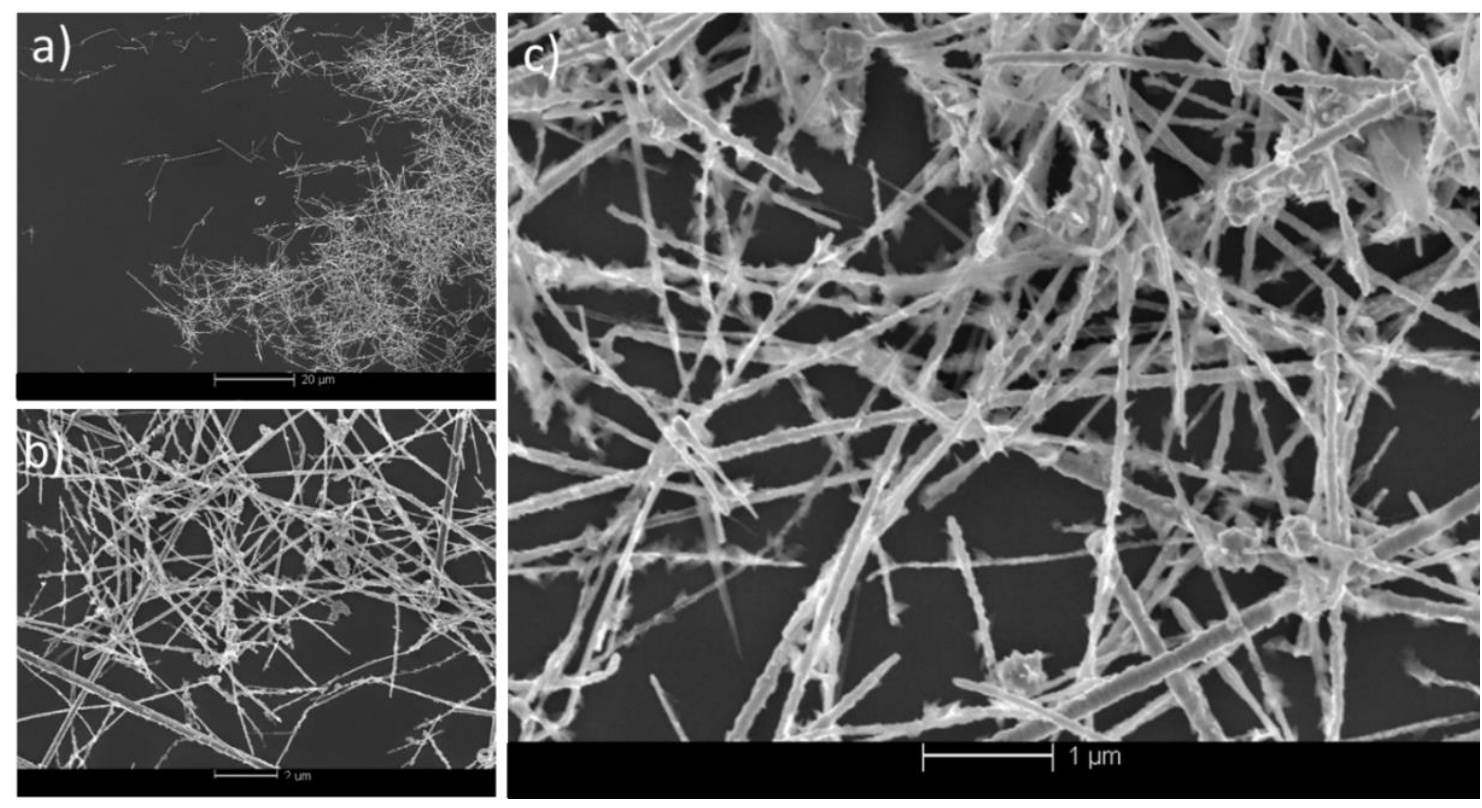

Figure 3.4. SEM images of copper nanowires synthesized using an intermediate concentration of hydrazine $\left(8.28 \times 10^{-3} \mathrm{M}, 30 \mu \mathrm{L}\right)$.

At an intermediate concentration of hydrazine $\left(8.28 \times 10^{-3} \mathrm{M}, 30 \mu \mathrm{L}\right)$, the synthesis yielded nanowires surface decorated with comparatively small particles or spikes; See 
Figure 3.4. As the hydrazine concentration was further reduced to $5.73 \times 10^{-3} \mathrm{M}(21 \mu \mathrm{L})$, the resulting NW exhibited high aspect ratio ( 250) and a particle-free and comparatively smooth surface, see SEM images in Figure 3.5a \& b. The average length and the diameter of NWs was $\sim 30 \pm 4 \mu \mathrm{m}$ and $120 \pm 20 \mathrm{~nm}$, respectively. The t-EBSD pattern of the same batch of NWs, shown in Figure 3.5c \& d, suggested a comparatively smooth and single crystalline surface. The dark regions in the orientational mapping images represented poor electron transmission. 

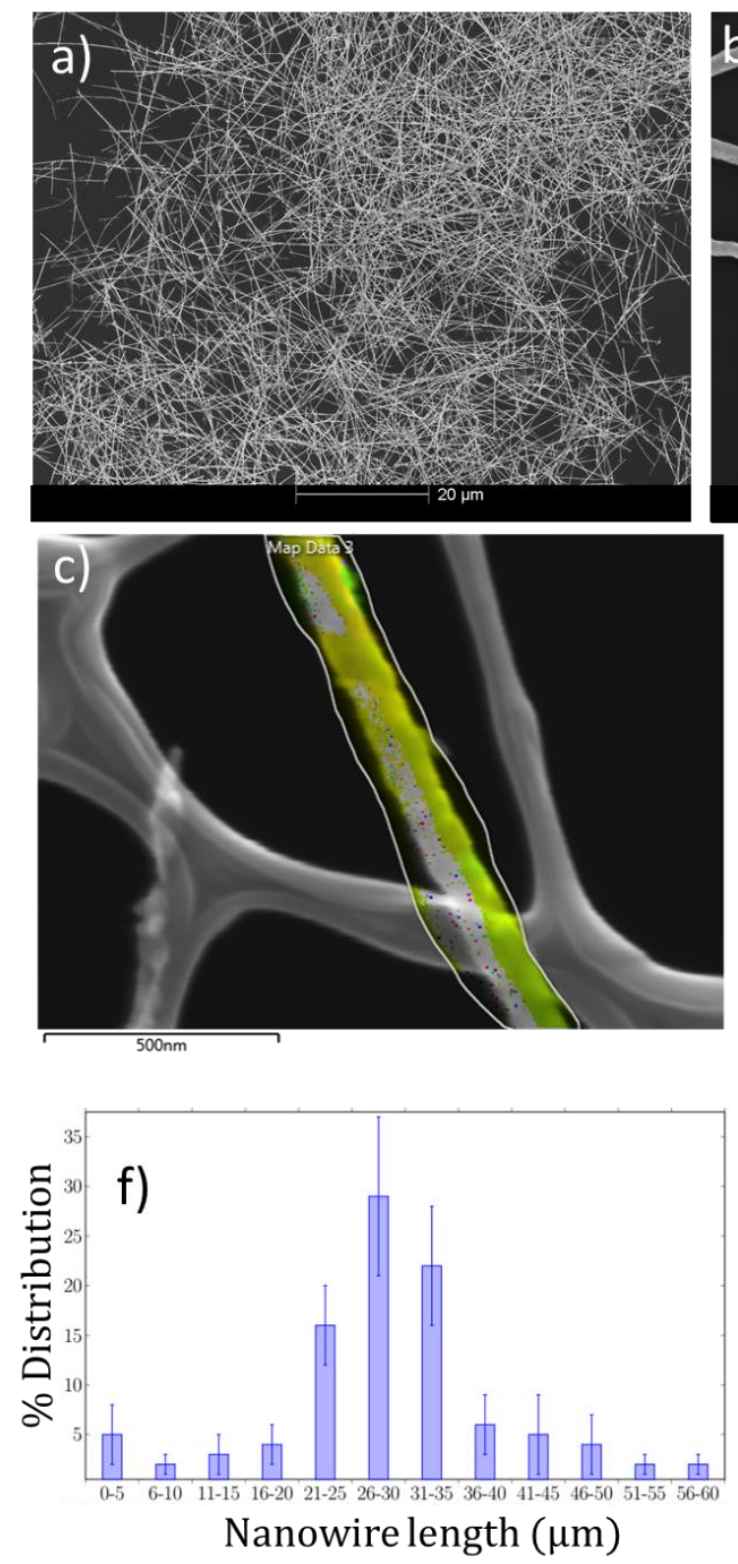

d)

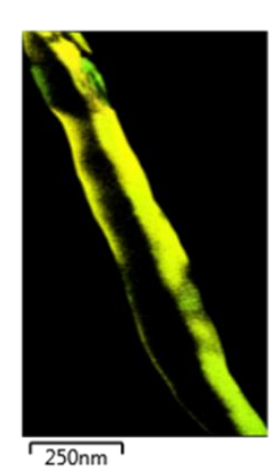

e)
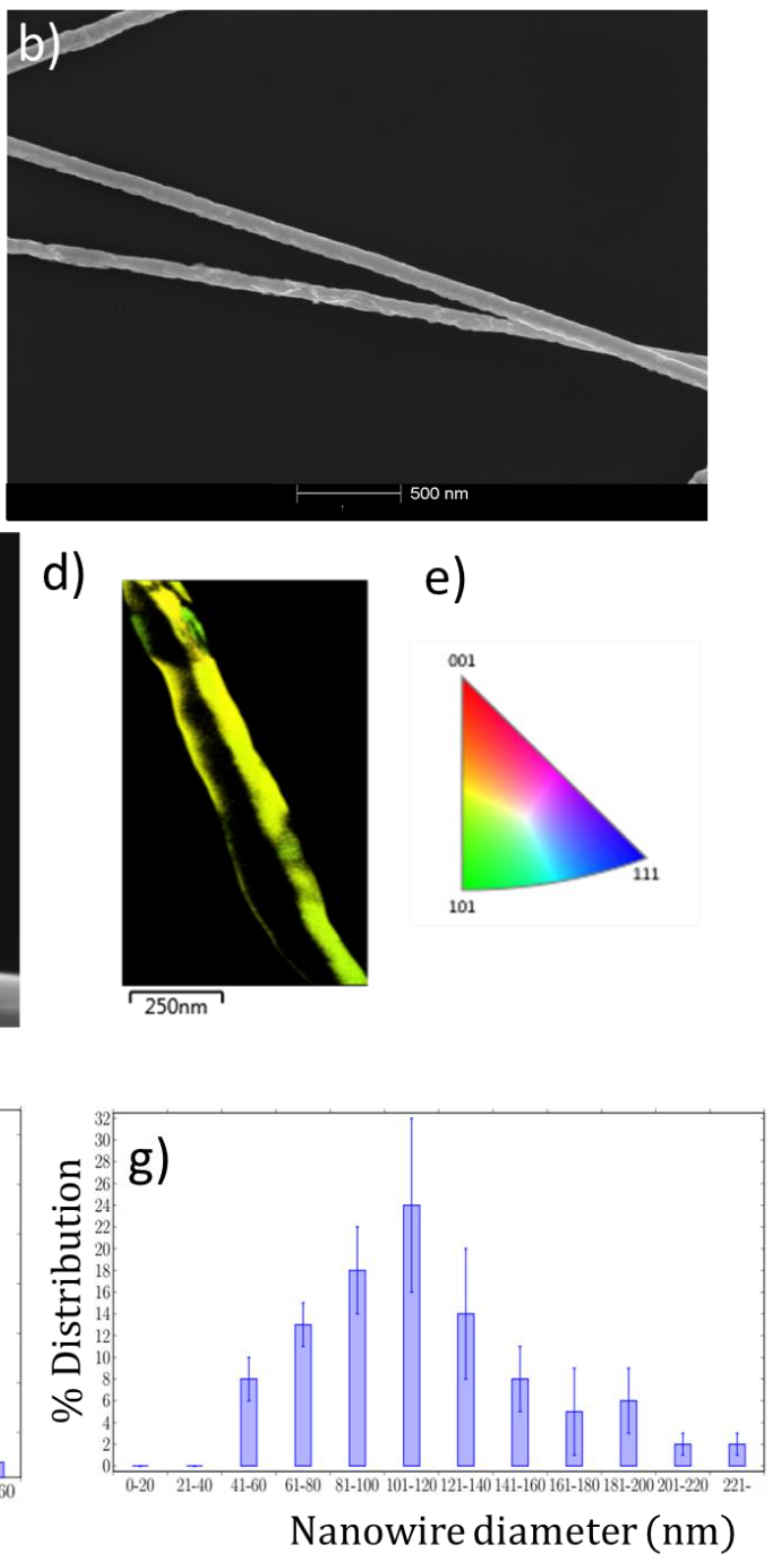

Figure 3.5. SEM images of $\mathrm{Cu}$ NWs synthesized using a low concentration of reducing agent shown at low a) and high b) magnification scales. TKD c), and out-of-the-page (Z) direction crystallographic orientation in d), showing particle free thin Cu NWs. And, e) showing a color map for crystallographic planes. Histograms in $\mathrm{f}$ ) and g) represents the length and diameter distributions of as-synthesized $\mathrm{Cu}$ NWs extracted from samples containing >200 NWs. 
Low magnification TEM image of the same batch of NWs (hydrazine concentration $5.73 \times 10^{-3} \mathrm{M}, 21 \mu \mathrm{L}$ ) shown in Figure 3.6 revealed the thinner and particle free nature of the $\mathrm{Cu}$ NW surface. As the hydrazine concentration was further decreased to $4.09 \times 10^{-3} \mathrm{M}$ $(15 \mu \mathrm{L})$, it resulted in a very poor yield of $\mathrm{Cu}-\mathrm{NWs}$ with low aspect ratios. Also, a large portion of the reaction mixture remained in blue, which indicated that the hydrazine concentration was not sufficient to reduce all the copper ions.

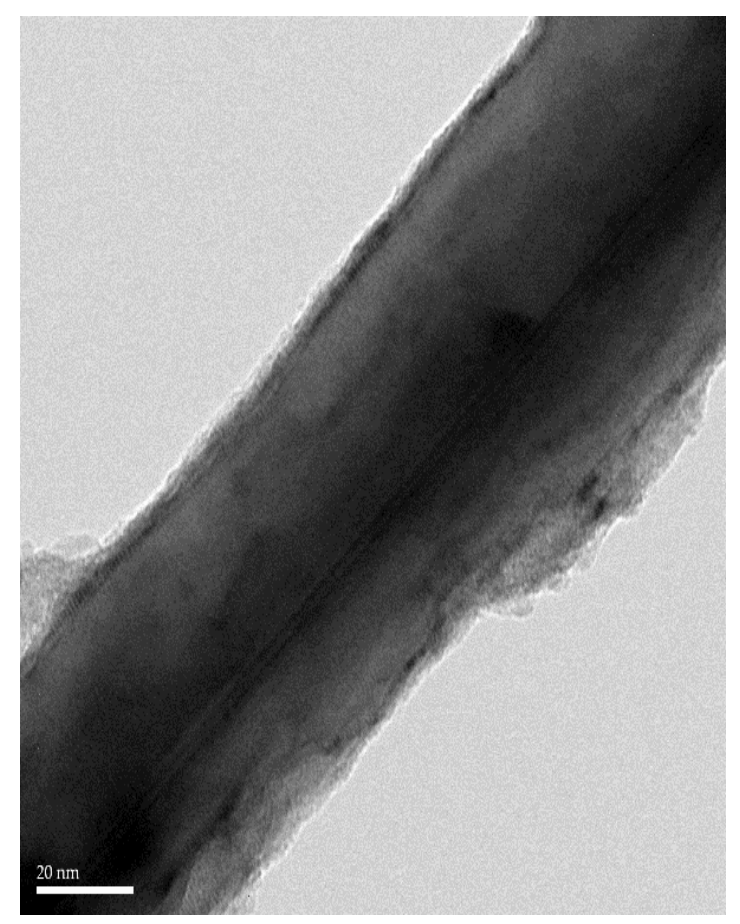

Figure 3.6. TEM image of $\mathrm{Cu} N W$ synthesized using $5.73 \times 10^{-3} \mathrm{M}(21 \mu \mathrm{L})$ concentration of hydrazine highlighting the particle-free natured surface.

The following three models could explain the formation of these particle or spike decoration on the NWs surface. First one is based on the screw dislocation-driven growth model discussed by Song Jin et $\mathrm{al}^{68}$. In the reaction, copper ions $\left(\mathrm{Cu}^{2+}\right)$ form complexes with $\mathrm{OH}^{-}$ions $\left(\mathrm{Cu}(\mathrm{OH})_{4}{ }^{2-}\right)$ and ethylenediamine $(\mathrm{EDA})\left(\left[\mathrm{Cu}(\mathrm{en})_{2}\right](\mathrm{OH})_{2}\right)$ and presence of 
these complex ions cause a decrease in the supersaturation of nanowire anisotropic growth. When an excess of the reducing agent results in a reduction of these copper complex ions into copper atoms all at once, a decrease in these complex ion concentrations causes an increase in the supersaturation at the tip of the nanowires ${ }^{68}$. Hence, NWs stop growing in the axial direction and promotes the growth of nanowire in the lateral axis and may form particles with various crystal planes which are grown at the screw dislocation sites on the nanowire surface. Whereas at moderate hydrazine concentration, the presence of copper complex ions decreases the supersaturation and hence causes the growth of NWs in longitudinal axis leading to high aspect ratio NWs.

The second hypothesis is based on the EDA as a facet selective promoter of NW growth mechanism which is discussed by Wiley et, $\mathrm{a}^{69}$. Presence of excess hydrazine keeps the NW surface free of $\mathrm{Cu}$ oxide and promotes deposition of copper on the NW surface which results in thicker NWs. Whereas at a moderate concentration of hydrazine, the NW surface remains oxidized due to the presence of high $\mathrm{OH}^{-}$ions and prevents the growth in NW lateral axis direction and promotes the longitudinal axis ((100) facet) growth which ultimately results in high aspect ratio NWs.

The third hypothesis is an excess of reducing agent concentration results in a reduction of copper ion complexes to copper atoms all at once, and these highly concentrated copper atoms agglomerate to form multiply twinned nanoparticles. TEM images shown in Figure 3.7 a \& b shows the presence of nanoparticles in the solution along with NWs, and perhaps during the nanowire growth, some of the particles may stick onto the nanowire surface leading to NW thickening. See Figure 3.7c. The mechanism reported 
by Wiley et, al. supports this hypotheses ${ }^{33}$. The exact reason behind the particle decoration is not completely understood for now. Nevertheless, experimental studies show how the concentration of reducing agent affects the morphology and allows the determination of the optimized concentration to synthesize high aspect ratio and particle free nanowires.
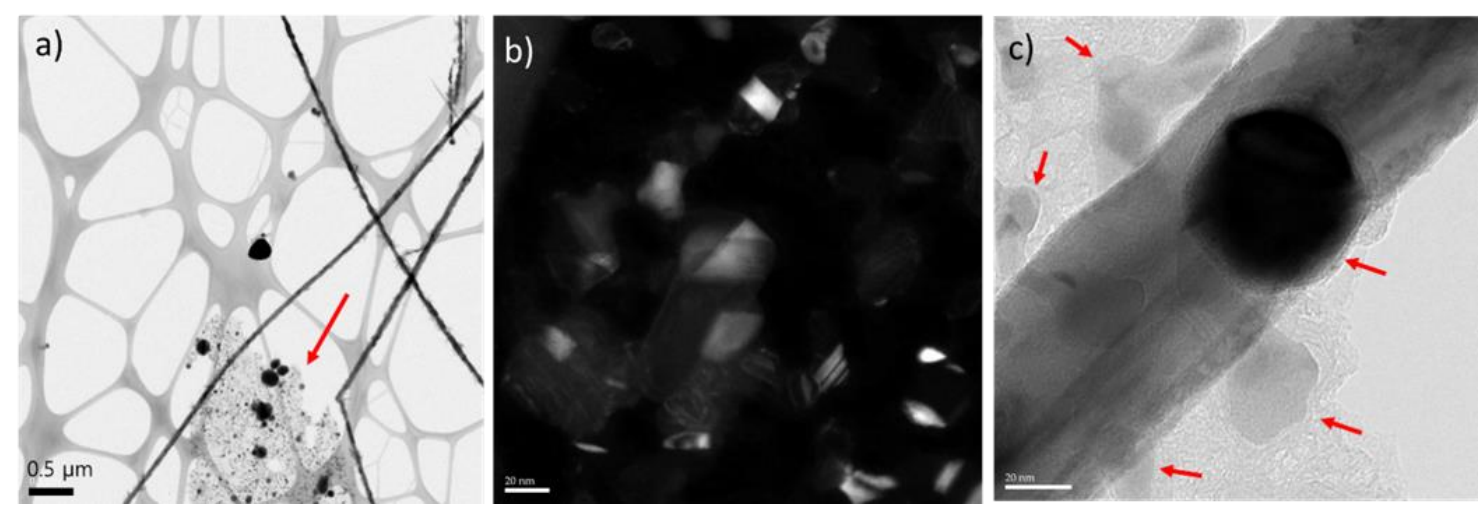

Figure 3.7. Low magnification bright field a), and high magnification dark field b), TEM image showing the presence of nanoparticles. Bright field image c), showing nanoparticles sticking to the surface of the nanowire at multiple sites (pointed out with arrows).

\subsection{Electroless plating of nickel (Ni) on Cu NWs surface}

3.2.1. Nickel electroless plating procedure: Electroless plating of nickel onto the $\mathrm{Cu}$ NWs surface was conducted by a slightly modified version of the method discussed by Wiley et al. ${ }^{59,72}$. In brief, the procedure involved mixing of $\mathrm{Ni}\left(\mathrm{NO}_{3}\right)_{2}(0.1 \mathrm{M}$ in DI water, $0.4 \mathrm{~mL})$ and hydrazine $(0.16 \mathrm{M}(600 \mu \mathrm{L}))$ reagents with a $10 \mathrm{mg}$ of Cu NWs (dry mass) suspension in ethylene glycol $(20 \mathrm{~mL})$. The above solution was thoroughly vortex mixed for 5 minutes and heated in an oil bath at $120^{\circ} \mathrm{C}$. After heating for 10 minutes, the solution turned clear and dark-colored nickel coated $\mathrm{Cu}$ NWs (Cu/Ni NWs) floated at the air-water interface due to trapped $\mathrm{N}_{2}$ gas bubbles generated in the reaction, See Figure 3.8. 

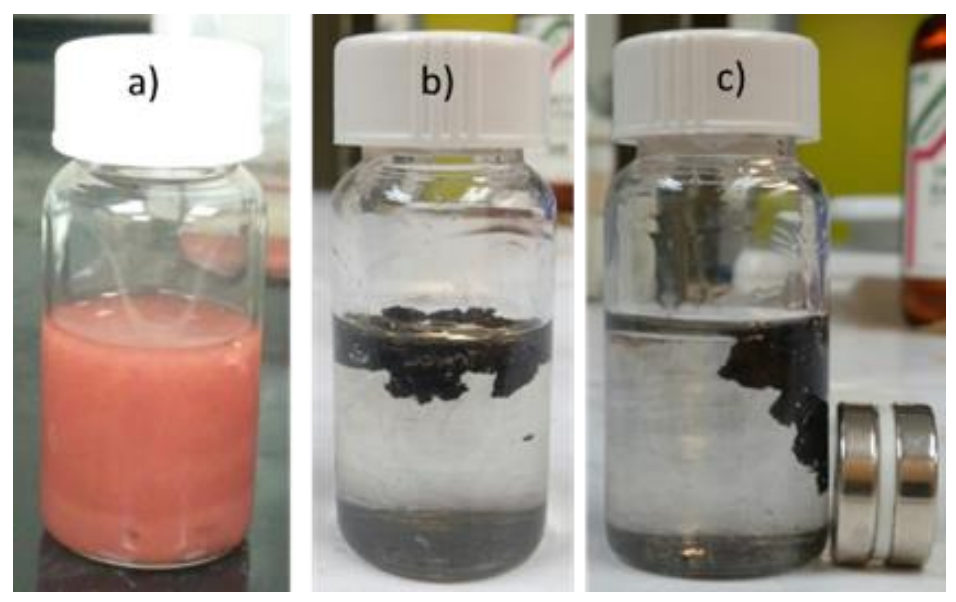

Figure 3.8. Digital photographs are showing nickel electroless plating mother solution (a mixture of $\mathrm{Cu} \mathrm{NWs} / \mathrm{Ni}\left(\mathrm{NO}_{3}\right)_{2} /$ Hydrazine in ethylene glycol solvent) a). After heating at $120{ }^{\circ} \mathrm{C}$ for 10 minutes, floating of dark-colored nickel coated $\mathrm{Cu}$ NWs at the air-water interface can be observed in b), and magnetic response of $\mathrm{Cu} / \mathrm{Ni}$ NWs towards neodymium disc magnets is shown in $\mathrm{c}$ ).

When a disc magnet was placed next to the freshly prepared $\mathrm{Cu} / \mathrm{Ni} \mathrm{NW}$ s solution, it attracted the mass of dark cupronickel nanowires, see Figure 3.8c. The nickel coating thickness on $\mathrm{Cu}$ NWs surface was studied by using EDS elemental mapping, See Figure 3.9, which revealed nickel layer on the CuNW surface of $\sim 20 \mathrm{~nm}$ thickness. Also, the presence of oxygen at the interface of $\mathrm{Cu}$ and $\mathrm{Ni}$ indicated oxidized copper NW surface. But the nickel surface did not show significant oxidation implying metallic surface.

Electroless coating of nickel would be advantageous in a few aspects, such as protecting the core copper from further oxidation and for reducing the contact resistance between nanowires due to strong binding resulting from the ferromagnetic interaction. Furthermore, the ferromagnetic nature of the nickel allowed a directional control over the orientation of the NW by applying an external magnetic field. 

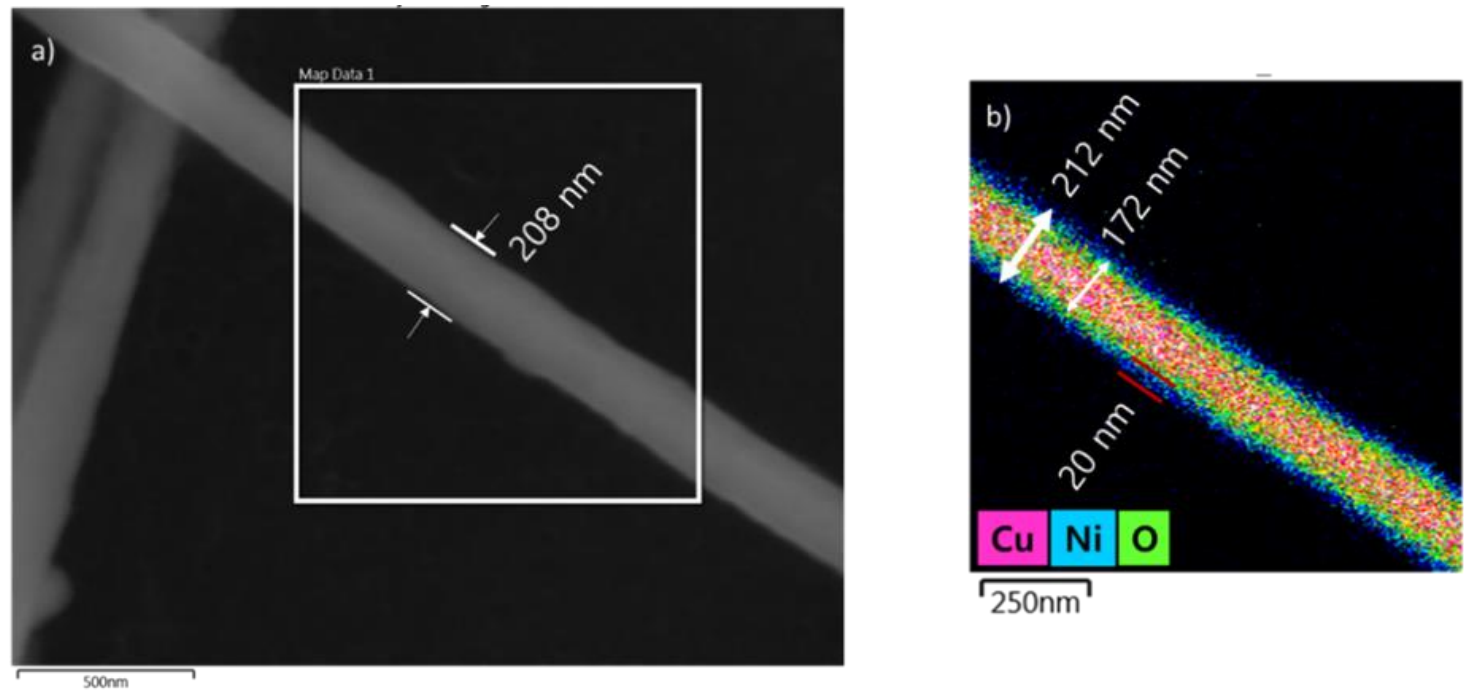

Figure 3.9. SEM image of nickel coated copper nanowire ( $\mathrm{Cu} / \mathrm{Ni} \mathrm{NW})$ shown in a), and elemental mapping image showing the distribution of copper core and nickel coating on the surface b). In between the copper and nickel layers copper oxide (green color) can be observed.

\subsection{Magnetic field assisted alignment}

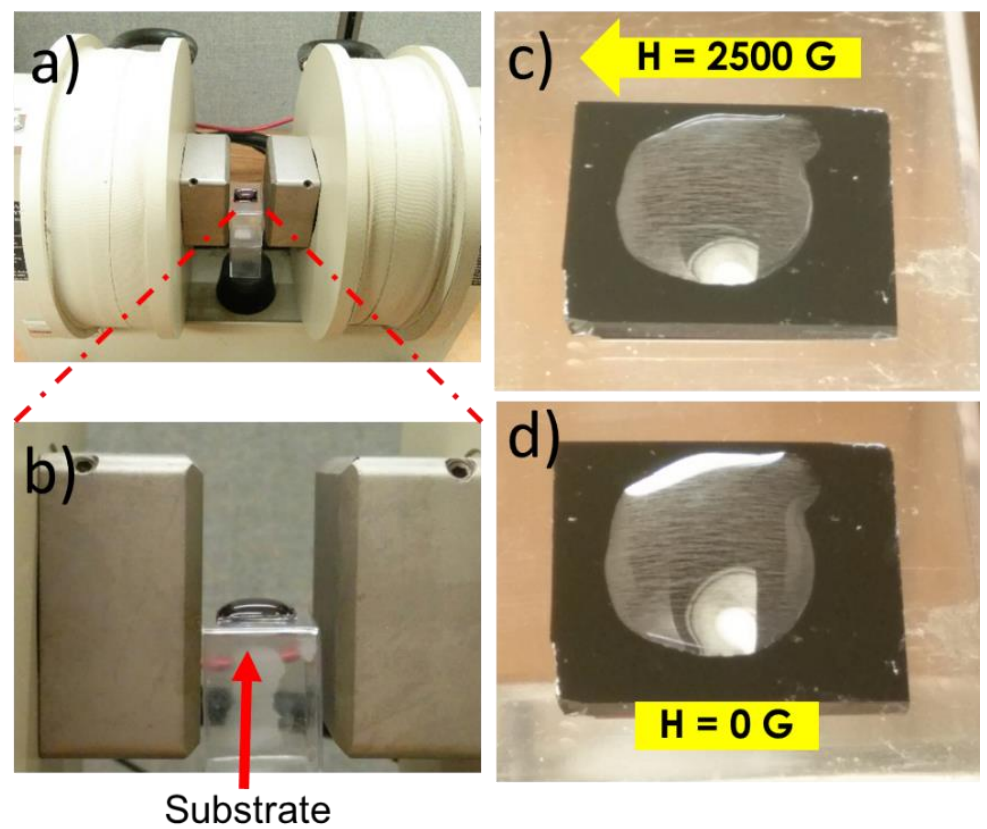

Figure 3.10. Digital photographs of electromagnet setup (GMW, Model-3470) used for controlling the orientation of $\mathrm{Cu} / \mathrm{Ni} \mathrm{NWs}$ are shown in a) and b). Digital photograph of well-aligned NWs in solution at $2500 \mathrm{G}$ of applied magnetic field and after turning off the magnetic field to $\mathrm{H}=0 \mathrm{G}$, no rearrangement of NWs chains can be observed in d). 
Controlling the orientation of nanowires is very critical for the assembly of NWs into complex nano or microelectronic devices. Here the magnetic field assisted alignment was explored to systematically control the orientation of $\mathrm{Cu} / \mathrm{Ni} \mathrm{NWs}$ on a centimeter scale area. The GMW electromagnet system provided the uniform magnetic field in between its poles (see Figure 3.10). The alignment procedure involved deposition of the $\mathrm{Cu} / \mathrm{Ni}$ NWs aqueous solution on a given substrate as shown in Figure 3.10 to align NWs aligns in the applied magnetic field direction. Each NW effectively acted like a nanobar magnet in the field and formed NWs chains by connecting with each other in a head to tail fashion. And, surprisingly even after turning off the magnetic field $(\mathrm{H}=0 \mathrm{G}) \mathrm{NWs}$ chains remained in a well-organized manner, see Figure $3.10 \mathrm{~d}$. This suggests owing to the ferromagnetic, (not paramagnetic) nature of nickel all the NWs remained magnetized. This led to a strong binding to each other even after turning off the magnetic field. These types of well-aligned nanowire arrays are very attractive for nanowire-based devices owing to its excellent percolation network formation, uniformity and comparatively lower NW-NW junction resistance ${ }^{45}$.

Figure 3.11 shows the optical microscopic images of $\mathrm{Cu} / \mathrm{Ni}$ NWs alignment in the presence and the absence of an applied magnetic field. When the NWs solution was allowed to dry under ambient conditions in the absence of a magnetic field, typically all the NWs arranged in random directions forming agglomerations due to Van der Waal forces of attractions. However, when the external magnetic field was applied, all the NWs acted like nanobar-magnets and aligned in the applied magnetic field direction, See Figure 3.11b. Furthermore, strings of NWs formed suggesting end-to-end attachment of NWs due 
to favorable magnetic interaction (i.e., the attraction between north and south poles of the neighboring NWs). Following two steps of deposition and alignment in orthogonal directions produced an NW mesh or crossed arrays (Figure 3.11c) useful for fabricating flexible and transparent electrode preparation ${ }^{45,49}$.

Systematic studies of alignment phenomenon were undertaken. In the absence of a magnetic field, all the NWs arranged in the random directions with an average angle of $45^{\circ}$ with respect to the hypothesized axis and the resulting deposits showed agglomerations. Whereas, with an applied magnetic field $(2500 \mathrm{G})$, the majority of the NWs aligned with an angle less than $10^{\circ}$ with respect to the applied magnetic field direction (see angular distribution in Figure 3.11d). The NWs alignment angle distribution with respect to the applied magnetic field direction (in case of a magnetic field applied) and hypothesized axis (in case of no magnetic field applied) were determined by using Image-J application, see Appendix-B for a detailed protocol. Also, the average angle with respect to the magnetic field direction depended on the field strength. Therefore, the alignment distribution function was determined as a function of the magnetic field strength that varied from $0 \mathrm{G}$ to $2500 \mathrm{G}$. 

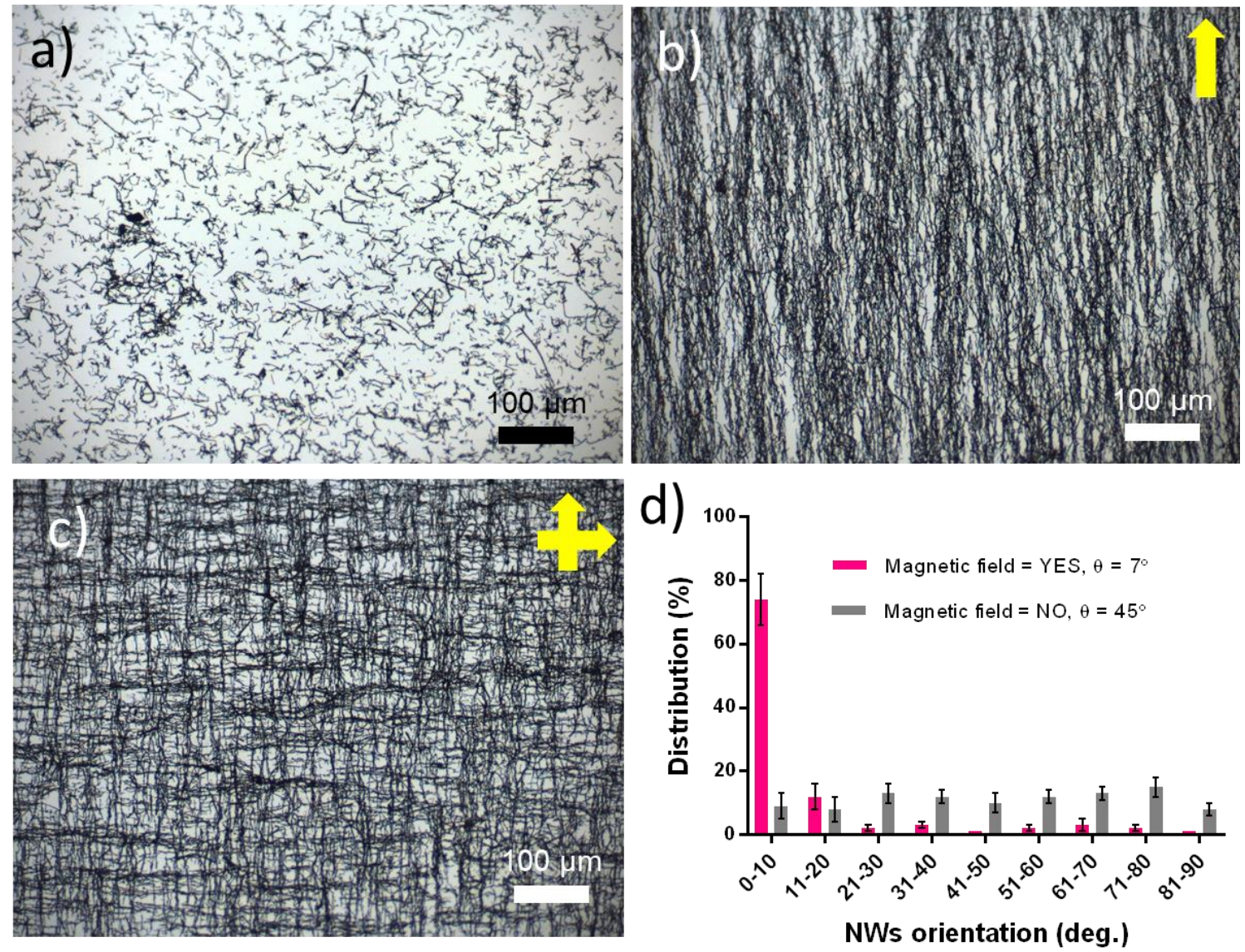

Figure 3.11. Optical microscope images of $\mathrm{Cu} / \mathrm{Ni} \mathrm{NWs}$ when they are allowed drying in the absence (a), and in the presence of applied magnetic field (2500 Gauss) (b). Image (c) shows the NWs mesh prepared by depositing a $2^{\text {nd }}$ layer of NWs orthogonally. The histogram in (c) highlights the NWs orientation distribution with respect to the applied magnetic field direction.

\subsubsection{Effect of magnetic field strength on NWs alignment order}

As discussed in the previous sections, each nickel coated $\mathrm{Cu} N W(\mathrm{Cu} / \mathrm{Ni} \mathrm{NW})$ acted like a nano bar-magnet and responded to the externally applied magnetic field. Alignment strength of these bar magnets was explored by depositing $100 \mu \mathrm{L}$ of $\mathrm{Cu} / \mathrm{Ni} \mathrm{NW}$ solution $(0.5 \mathrm{mg} / \mathrm{mL})$ on the $1 \times 1 \mathrm{~cm}$ sized wafer substrate and the solvent was allowed to evaporate in the presence of a magnetic field with strength varying from 0 to $2500 \mathrm{G}$. See Figure 3.12. When there was no magnetic field $(0 \mathrm{G})$, all the NWs aligned in the random directions 
and formed agglomerations. As the magnetic field strength increased, $\mathrm{Cu} / \mathrm{Ni} \mathrm{NWs}$ began to form chains by connecting the NWs in an end-to-end fashion. As the field strength increased, NWs chain lengths tended to increase.

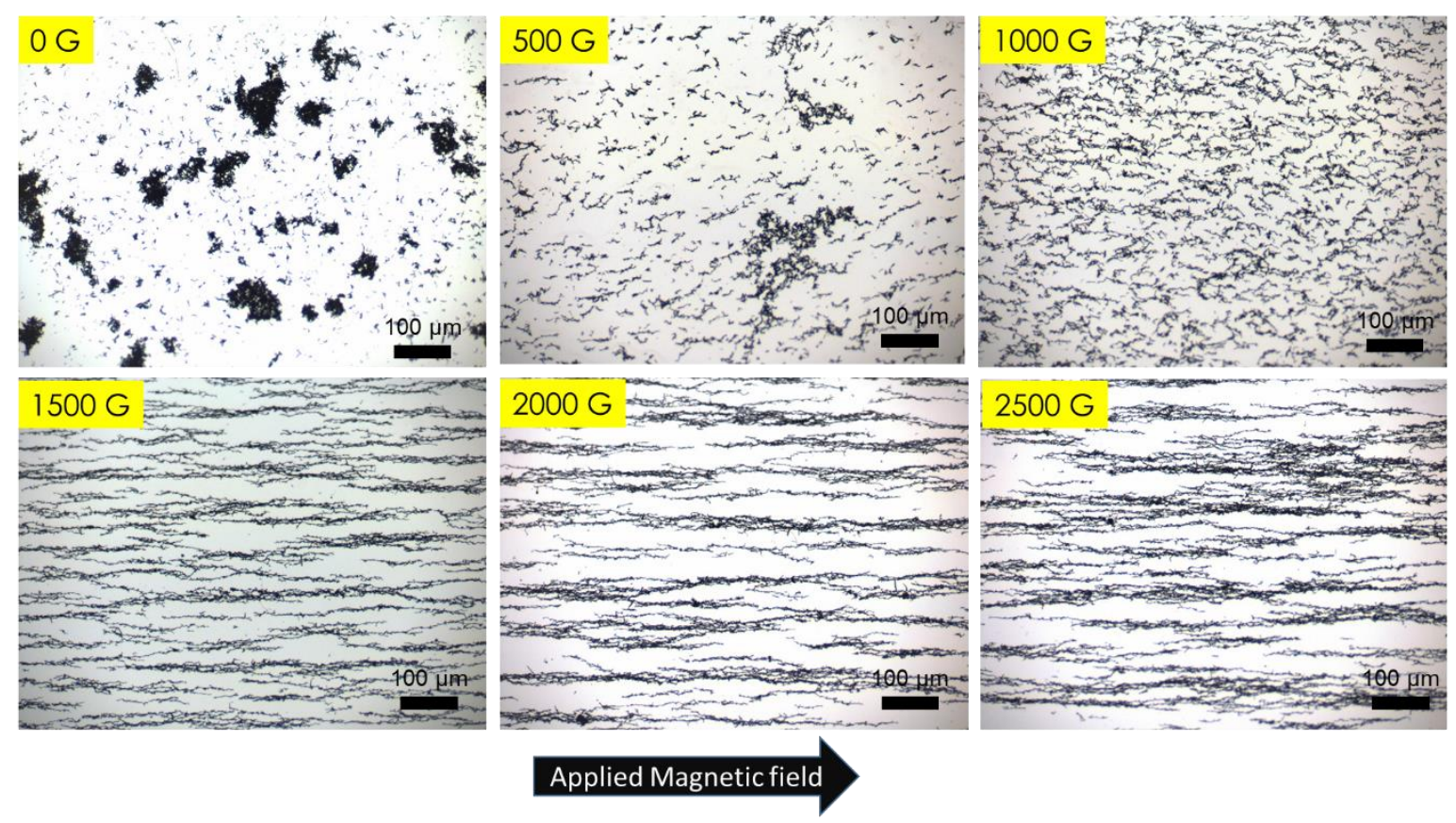

Figure 3.12. Optical micrographs showing alignment order of $\mathrm{Cu} / \mathrm{Ni} \mathrm{NWs}$ at various applied magnetic field strength from $0 \mathrm{G}$ to $2500 \mathrm{G}$.

Excellent alignment with an average alignment angle of $\sim 12^{\circ}$ was achieved at 1500G. As the field strength is further increased to the 2500G, the average alignment angle decreased to $\sim 8^{\circ}$. The observation suggested that at least a $1500 \mathrm{G}$ magnetic field strength is required to get a reasonably good alignment. The average alignment angle tended to be further reduced and reached saturation at higher field strengths, See Figure 3.13. The distribution function for the corresponding NWs is shown below along with a summary of parameters in the in the accompanying table. 


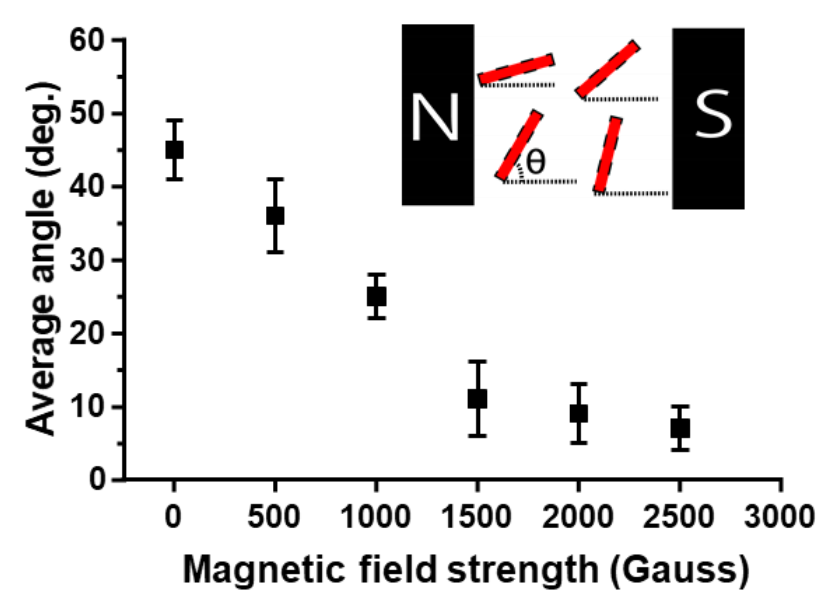

Figure 3.13. Plot showing average NWs alignment angle with respective to the applied magnetic field direction, at various field strength from $0 \mathrm{G}$ to $2500 \mathrm{G}$. The inset image describes the alignment angle definition.
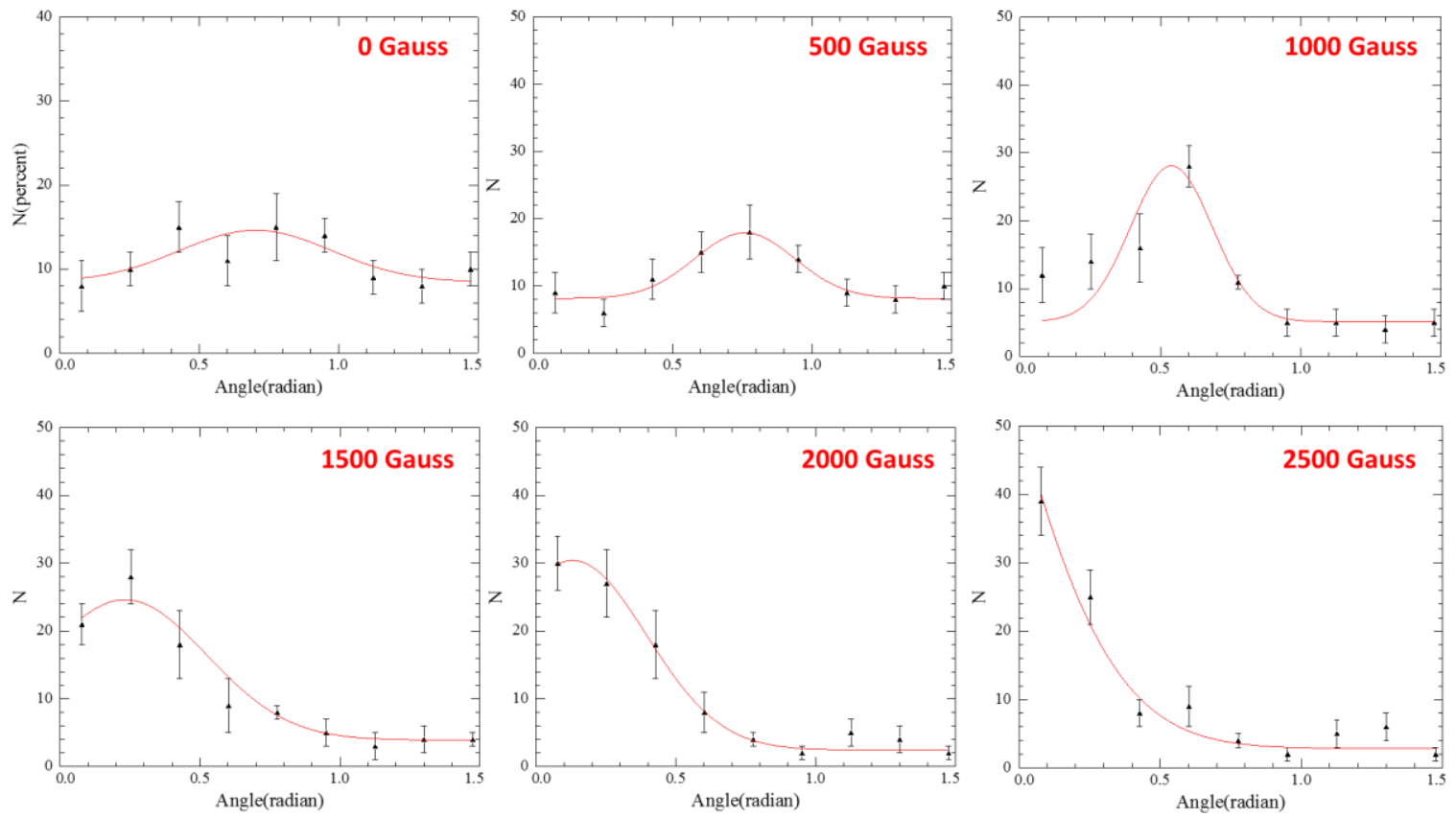

Figure 3.14. Gaussian peak fitting plots are showing NWs alignment distribution with respect to the applied magnetic field strength from 0 to 2500 Gauss.

Orientation distribution histograms of $\mathrm{Cu} / \mathrm{Ni} \mathrm{NWs}$ measured as a function of magnetic field strengths were fitted to a Gaussian equation below. 


$$
N(\theta)=A e^{-\left(\frac{(\theta-<\theta>)}{\Delta \theta}\right)^{2}}+B
$$

$A$ and $B$ are a normalization constant and a constant background fitting parameter. $N(\theta)$ is a number of nanowires oriented at angle $\theta$ in a bin size of 0.2 radians. $<\theta>$ and $\Delta \theta$ are the average absolute value of the NW orientational angle and the width of the distribution respectively. The best-fit parameters and respective curve fitting uncertainties appear in Table 3.2.

\begin{tabular}{|l|l|l|}
\hline $\begin{array}{l}\text { Field } \\
\text { Strength(gauss) }\end{array}$ & $\theta($ radian $)$ & $\Delta \theta$ (radian) \\
\hline 000 & $0.7 \pm 0.1$ & $0.4 \pm 0.2$ \\
\hline 500 & $0.75 \pm 0.05$ & $0.25 \pm 0.08$ \\
\hline 1000 & $0.54 \pm 0.03$ & $0.21 \pm 0.03$ \\
\hline 1500 & $0.23 \pm 0.07$ & $0.41 \pm 0.08$ \\
\hline 2000 & $0.13 \pm 0.09$ & $0.37 \pm 0.09$ \\
\hline 2500 & $0.3 \pm 0.7$ & $0.5 \pm 0.3$ \\
\hline
\end{tabular}

Table 3.2: List of field dependent parameters.

\subsection{Summary of copper nanowires synthesis and magnetic field assisted alignment}

Control over nanowire surface roughness, aspect ratio, and alignment are very important for their device application. In this thesis research, a solution based synthesis method was optimized with respect to the reducing agent concentration to synthesize the high aspect ratio NWs with a smooth surface. Also, the effect of synthesis temperature on the aspect ratio of nanowires was investigated. As the synthesis temperature increased, the aspect ratio of NWs decreased. In the case of controlling the alignment of $\mathrm{Cu} \mathrm{NWs}$, the previous researcher had reported very limited success via CVD, electrochemical deposition in a porous template and thermal assisted growth of vertically aligned NWs. For the first-time simple magnetic field based alignment of NWs on macroscopic wafer-scale was realized 
by coating $\mathrm{Cu}$ NWs with a thin layer of ferromagnetic nickel. The nickel coating would be further advantageous in protecting the copper core from oxidation. 


\section{CHAPTER FOUR}

\section{SURFACE DERIVATIZATION OF CARBON DOPED SILICON DIOXIDE (CDO) WAFER}

Carbon doped silicon dioxide (CDO), a highly nanoporous low- $k$ dielectric, was chosen as a substrate for the fabrication of nanowires based interconnects. The selfassembly of NWs into interconnect structures on $\mathrm{CDO}$ wafer required surface preparation of CDO to attract and strongly hold the nanowires after deposition. In this work, thiol ($\mathrm{SH})$ functionalization of the $\mathrm{CDO}$ surface and strong interaction of thiol to copper and nickel was explored. Thiol functionalization of the CDO surface was achieved via a novel approach developed to selectively functionalize the surface exposed silicon hydroxide ( $\mathrm{Si}$ $\mathrm{OH})$ functional groups ${ }^{60}$. The hydrophilic $\mathrm{Si}-\mathrm{OH}$ functional groups are generated during various steps in chip integration processing and cause undesirable moisture uptake which adversely increases the dielectric constant $(k)$ of CDO. We addressed this issue by selectively derivatizing the surface exposed hydrophilic $\mathrm{Si}-\mathrm{OH}$ functional groups to highly hydrophobic long alkyl chain (Si-R, R = octadecyl chain). The dense layer of octadecyl chains then acts as a moisture repellent thin film. This chapter provides the details of CDO film derivatization by octadecyl and thiol chains along with CDO surface characterization by XPS, IR, capacitance-voltage and contact angle techniques.

\subsection{Introduction}

Some of the biggest achievements in the past half-century involved the development of the microprocessor industry. To address a large number of transistors integrated into microprocessors, a sophisticated multilayer wiring, the so-called, interconnect architecture, 
was essential. In the beginning of the Integrated Circuit (IC) era, aluminum alloys, tungsten, and $\mathrm{SiO}_{2}$ insulators were the materials of choice for interconnects systems. In the early 1990s, researchers had realized the concept of improving the IC performance by increasing the transistor count and making changes to the traditional wiring and insulator materials ${ }^{11}$. As the ICs feature sizes were downscaling, $\mathrm{Al} / \mathrm{W} / \mathrm{SiO}_{2}$ interconnect system became the limiting factor due to high power consumption and low electromigration resistance. Owing to its advantageous high conductivity, abundance, and ductility characteristics, copper $(\mathrm{Cu})$ as a interconnect material was extensively explored for many years $^{73,74}$. In 1997, IBM made a remarkable contribution to IC industry, by developing chips with copper interconnects, that could significantly enhance the chip performance ${ }^{4,5}$, 75. Multilayered copper interconnect chip developed by IBM in 1997, is shown in Figure 4.1.

In comparison to aluminum, copper offers low electrical resistivity and high electromigration resistance. Using low resistivity interconnect material like copper decreases the RC signal delay and ultimately increases the IC speed. 


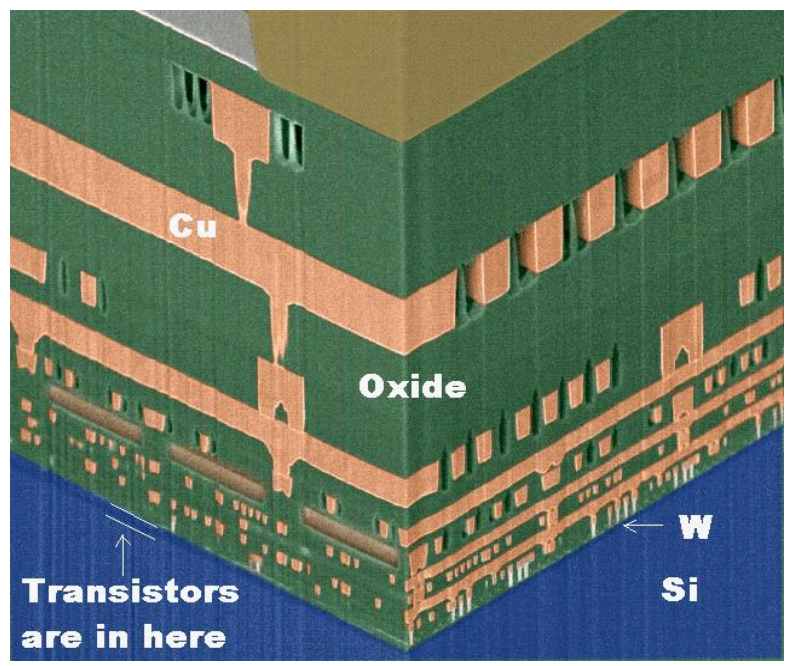

Figure 4.1. Image of the multilayered integrated circuit (IC) using $\mathrm{Cu} / \mathrm{W} / \mathrm{SiO}_{2}$ dielectric materials, developed by IBM in $1997^{4}$.

As the transistor count and interconnect density increase, the RC signal delay, and power dissipation also increase because of increased capacitive coupling between densely packed metal lines. Since the RC signal delay and power consumption all are directly related to dielectric constant $(k)$ of the inter-metal dielectric material, many efforts have been devoted to replacing the standard silicon dioxide $(k=3.9$ to 4.2$)$ with an ultra-low $k$ material $^{76}$. The Dielectric constant $(k)$ is defined as the ratio between permittivity of a substance to the permittivity of vacuum as shown in the below equation. In Table, $4.1 k$ values of common materials are shown.

$$
\text { Dielectric constant }(k)=\frac{\text { Permitivity of substance }}{\text { Permitivity of vacuum }}
$$

Due to low $k$ value, many organic compounds are considered as a good low $k$ dielectric material, but organics have limitations associated with poor thermal and mechanical stability. Furthermore, inorganic materials show good mechanical and thermal 
stabilities, but they possess higher $k$ and more serious moisture sensitivity, requiring an additional liner and capping layers ${ }^{77,78}$. Hybrid films, with organic and inorganic materials, have been a promising approach for low- $k$ materials with stable properties. Among hybrid type materials, carbon doped silicon oxide (CDO) material has attracted more attention owing to its low- $k$, and compatible mechanical and thermal properties ${ }^{3,79-82}$. Hence, in modern ultra-large-scale integrated circuit (ULSI) devices CDO has been a choice as a low- $k$ insulator.

\begin{tabular}{|l|l|}
\hline Substance & $k$-value \\
\hline Vacuum & 1 \\
Polyethylene & 2.25 \\
Carbon Doped Silicon Oxide (CDO) & 2.2 to 3.0 \\
$\mathrm{SiO} 2$ & 3.9 \\
Silicon & 11.68 \\
Water & $\sim 80$ \\
\hline
\end{tabular}

Table 4.1. Dielectric constant $(k)$ values of various substances.

\subsection{CDO as a low- $k$ material}

Over the last few years, the classic $\mathrm{SiO}_{2}$ insulator used to separate metallic interconnect lines are being replaced by materials of lower $k$. Lower $\mathrm{k}$ improves signal propagation delay. The lower the $k$ value shorter the propagation delay. Strategies to realize lower $k$ materials include (1) Introduction of carbon in $\mathrm{SiO}_{\mathrm{x}}$ network structure, by using so-called CDO materials (reviewed in this work). CDO film preparation and chemical structures etc. are discussed in detail in the following sections. (2) Incorporating porosity (note: air has lowest $k \sim 1$ ). Porosity is inversely related to $k$-value; as the porosity 
increases, $k$ values decreases. In the as deposited CDO film, nanopores can be introduced by plasma curing processes. The plasma burns off organic moieties and leaves nanopores in the film.

\subsection{Review on CDO film preparation and chemical structure}

Carbon doped silicon oxide (CDO), composed of $\mathrm{Si}, \mathrm{C}, \mathrm{O}$, and $\mathrm{H}(\mathrm{SiCOH})$, is deposited on Si (100) surface by plasma enhanced chemical vapor deposition (PECVD) from mixtures of silicon and an organic precursor ${ }^{80}$. In a PECVD reactor, the plasma is sustained by $13.56 \mathrm{MHz}$ rf power. The silicon and organic precursors are carried by He gas into the plasma chamber. SiCOH film preparation employs various silicon precursors such as tetramethylsilane $e^{80}$, hexamethyldisiloxane ${ }^{81}$, tetramethyltetrasiloxane ${ }^{82}$. In the presence of plasma, precursor molecules break down into fragments and deposit on the Si wafer. The deposited films are annealed at $400{ }^{\circ} \mathrm{C}$. The annealing step removes thermally unstable organic fragments and creates porosity in the material which enables the adjustment of the $k$ between 2.8 to 2.05. Post thermal treatment also causes extensive cross-linking of $\mathrm{Si}, \mathrm{C}$, and $\mathrm{O}$ to form $\mathrm{Si}-\mathrm{Si}, \mathrm{Si}-\mathrm{O}-\mathrm{Si}, \mathrm{Si}-\mathrm{CH}_{2}-\mathrm{Si}$ and $\mathrm{Si}-\mathrm{CH}_{2}-\mathrm{O}-\mathrm{Si}$ moieties. The annealing process depending on the duration and temperature causes the loss of $-\mathrm{CH}_{n^{-}}$groups and results in the formation of -Si-C- linkages and nanopores in the film ${ }^{83}$.

An alternative method to thermal annealing is plasma curing which can reduce $k(k=$ 2.0 to 2.2). The plasma curing process creates nanopores and improves the crosslinking which helps in improving the mechanical strength of the film ${ }^{84}$. The plasma curing process is an industry preferred method, owing to its advantageous lower operating temperature, 
uniformity, cost-effectiveness, shorter processing time, and produces improved mechanical strength of the film.

\subsection{Review on CDO damage and repairing methodologies}

\subsubsection{Review on CDO damage by plasma processing}

Interconnects in modern ICs integrate multilayers of copper wiring and insulating low $k$ materials (See Figure 4.1). They are fabricated using a combination of optical lithography and copper electroplating in a process known as the Dual Damascene (DD) $\operatorname{method}^{37}$. In optical lithography, a photoresist film made up of organic polymers, and photosensitive compounds serve as a patternable and sacrificial stencil. An optical image of the desired circuit pattern of trenches and vias are projected on the photoresist film coated on top of the CDO layer. Photochemical reactions in the light exposed regions alter the solubility of the photoresist film regio-specifically. In the developer solution, the film undergoes preferential dissolution (positive tone) or dissolution inhibition (negative tone) to expose the underlying CDO transferring an image of the desired circuit pattern on the chip. 

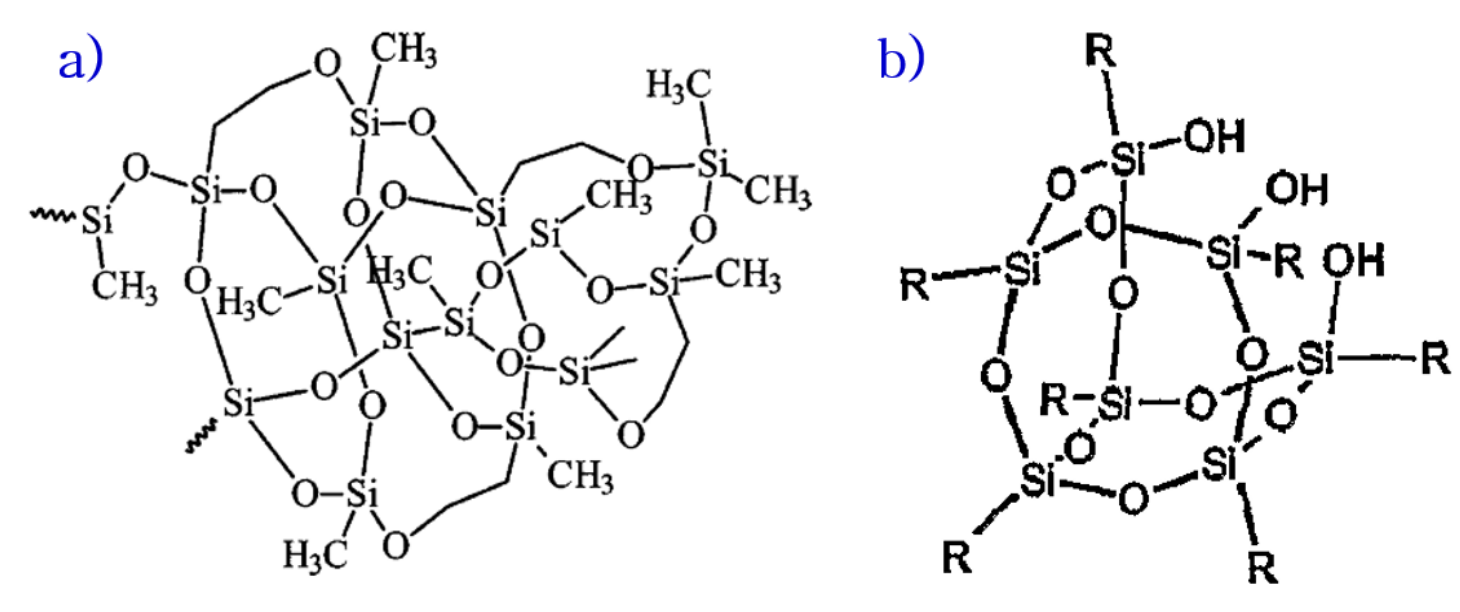

Figure 4.2. a) Chemical structure of porous cage networked structure of as-prepared CDO film from TMCTS precursor ${ }^{82}$, and $b$ ) chemical structure of plasma damaged film (R, represents an extension of Si-O-Si backbone structure) $)^{85}$.

This pattern is etched into the exposed CDO through plasma etching which opens up the electrical contacts in the lower layer. Subsequently, metallic copper is selectively and electrochemically plated in the etched vias/trenches, while avoiding its deposition on top of the undissolved electrically insulating photoresist film. After electroplating, the remaining sacrificial photoresist film is subjected to oxygen plasma ashing to remove organics as $\mathrm{CO} / \mathrm{CO}_{2}$ and water as ultimate byproducts ${ }^{86}$. Furthermore, oxygen radicals generated during the plasma processing react with the functional groups of the CDO film, breaking $\mathrm{Si}-\mathrm{CH}_{3}, \mathrm{Si}-\mathrm{H}$ bonds, and creates dangling bonds of silicon. These dangling bonds can easily react with hydroxyl ions in the plasma environment to form Si-OH bonds (see Figure 4.2)) ${ }^{85}$. The resulting increase in the number of hydrophilic $\mathrm{Si}-\mathrm{OH}$ groups in the CDO films makes the film more polar, hydrophilic and allows moisture uptake (water $k=$ 80). Absorbed moisture increases the $k$ value (note the dielectric constant of water is 80 !) 
and leakage current (due to protonic conduction) thereby compromising the quality of the $\mathrm{CDO}^{87}$.

\subsubsection{Review on CDO repairing methodologies}

Several approaches have been investigated to minimize the damage of porous CDO ultra-low $k$ film. One method involves protecting the CDO film from oxygen plasma damage by depositing a thin inert $\mathrm{SiN}_{\mathrm{x}}$ passivation layer. The $\mathrm{SiN}_{\mathrm{x}}$ film is generated from a thin layer of hybrid-organic-siloxane-polymer (HOSP) subjected to $\mathrm{NH}_{3}$ plasma treatment ${ }^{88}$. This inert layer can enhance the resistance of the HOSP film to moisture uptake and $\mathrm{O}_{2}$ plasma attack. Helium $(\mathrm{He})$ plasma treatment as a post $\mathrm{CDO}$ deposition has also been investigated to reduce the $\mathrm{O}_{2}$ plasma damage on the CDO films ${ }^{89}$. Treating the $\mathrm{O}_{2}$ plasma damaged CDO with a hydrogen $\left(\mathrm{H}_{2}\right)$ plasma to repair the damage by reducing silanol to silicon hydride has also been explored ${ }^{89}$. However, $\mathrm{CDO}$ repairs by $\mathrm{He}, \mathrm{NH}_{3}$ and $\mathrm{H}_{2}$ plasma treatments cause a loss of CDO film thickness (by reducing porosity) and irreversibly modify the network of the $\mathrm{SiCOH}$ chemical structure. Etching of plasma damaged low $k$ CDO layer by dilute HF solution has been investigated, but the concentration of HF solution and duration of treatments are very critical to prevent further etching of the undamaged CDO layer ${ }^{90}$.

Another approach to preventing moisture uptake in the damaged CDO is to convert surface silicon hydroxyl $(\mathrm{Si}-\mathrm{OH})$ functional groups to hydrophobic $\mathrm{Si}-\mathrm{O}-\mathrm{Si}\left(\mathrm{CH}_{3}\right)_{3}$ moieties by treating with hexamethyldisilazane (HDMS) ${ }^{62}$. However, the resulting single carbon ($\mathrm{CH}_{3}$, Methyl) termination is too thin to prevent the further attack from oxygen and moisture. Hence, it is necessary to find a way to selectively derivatize hydrophilic 
functional groups $(\mathrm{Si}-\mathrm{OH})$ to hydrophobic, without disturbing the backbone structure of the CDO film.

\subsection{Results of developed pathway for selective derivatization of silanol}

\subsubsection{Developed scheme for surface derivatization}

A gentle and spatially selective approach for the modification of surface exposed, hydrophilic silicon hydroxyl (Si-OH) functional groups to hydrophobic hydrogenterminated $(\mathrm{Si}-\mathrm{H})$ is developed. The stability and surface hydrophobicity was further enhanced by long alkyl chain derivatization of the silicon hydride $\left(\mathrm{Si}-\mathrm{R}, \mathrm{R}=\mathrm{C}_{18} \mathrm{H}_{37}\right)$ as shown in Scheme 4.3. Compared to methyl groups, long chain alkyl groups form a thicker and more compact hydrophobic film that blocks moisture uptake effectively ${ }^{91}$. The significant advantage of this approach is that the selective derivatization occurs only on the exposed or surface silicon hydroxyl $(\mathrm{Si}-\mathrm{OH})$ groups of $\mathrm{CDO}$ film without damaging the rest of the nano-porous cage structure. Additionally, silicon hydride formed during the intermediate stage may be further derivatized with different reactants to provide new surface functionalities $^{92}$. For example, it provides a convenient route for the insertion of additional functional groups such as terminal thiol (-SH) or amine $\left(-\mathrm{NH}_{2}\right)$ alkene structure.

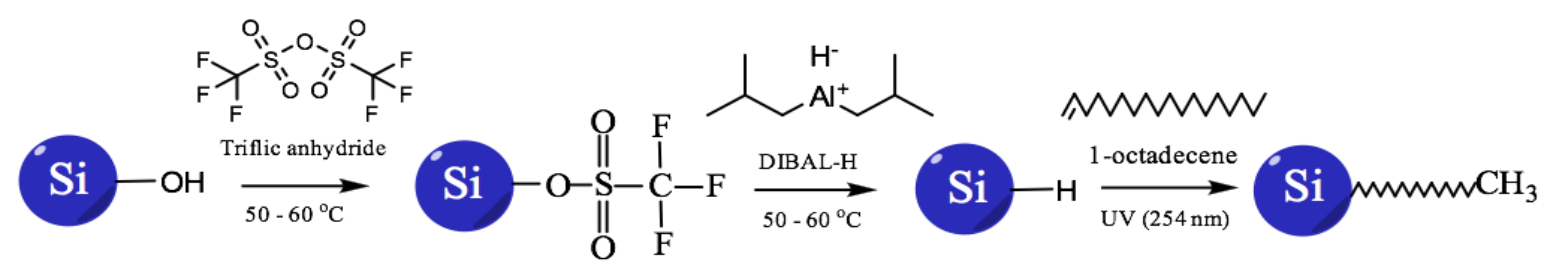

Scheme 4.1. Schematic of the new reaction pathway for hydrogen termination and octadecane (Si$\mathrm{R}, \mathrm{R}=\mathrm{C}_{18} \mathrm{H}_{37}$ ) derivatization. 
The viability of this approach has been established methodically by preliminary studies of derivatization of silanol groups in organic molecules, silica gel, $\mathrm{SiO}_{\mathrm{x}}$ wafer and $\mathrm{CDO}$ as shown in Figure 4.2 below.

a)

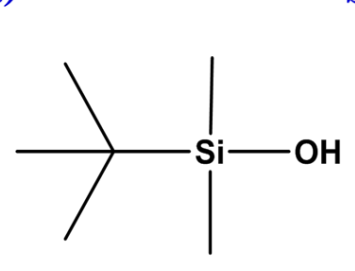

b)

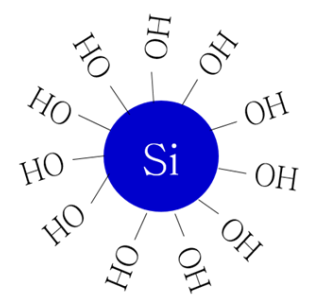

c)

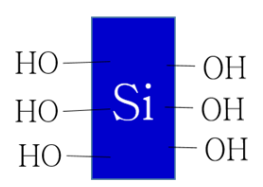

d)

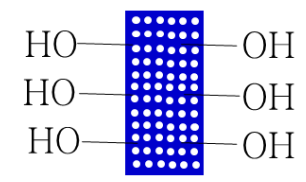

Scheme 4.2. Schematics of various compounds considered for testing surface derivatization reaction, a) tert-Butyldimethylsilanol b) silica gel, and c) $\mathrm{SiO}_{\mathrm{x}}$ wafer and d) nano porous carbon doped silicon oxide (CDO) wafer.

Common synthetic procedure: The first step in the reaction scheme employed $0.1 \mathrm{M}$ triflic anhydride $\left(\left(\mathrm{CF}_{3} \mathrm{SO}_{2}\right)_{2} \mathrm{O}\right)$ in anhydrous toluene at $50-60{ }^{\circ} \mathrm{C}$ for 48 hours to esterify the Si-OH functional group to yield silyltrifluoromethane sulfonate (Si-O-Tf, where Tf indicates the $\mathrm{SO}_{2} \mathrm{CF}_{3}$ group). In the second step, 0.1 M DIBAL-H (di-isobutylaluminum hydride, $\left.\left.(\mathrm{CH} 3)_{2} \mathrm{CHCH}_{2}\right)_{2} \mathrm{AlH}\right)$ reagent at $50-60{ }^{\circ} \mathrm{C}$ for 48 hours reduced the triflate intermediate $\left(\mathrm{Si}-\mathrm{O}-\left(\mathrm{SO}_{2}\right) \mathrm{CF}_{3}\right)$ to silicon hydride. Because hydrogen-terminated silicon is unstable, Si-H was further treated with 1-octadecene $\left(\mathrm{CH}_{3}\left(\mathrm{CH}_{2}\right)_{15} \mathrm{CH}=\mathrm{CH}_{2}\right)$ in the presence of UV light $(254 \mathrm{~nm})$ for 2 hours to produce $\mathrm{Si}-\mathrm{R}\left(\mathrm{Si}_{-} \mathrm{C}_{18} \mathrm{H}_{37}\right)$. All reactions were carried out in dry $\mathrm{N}_{2}$ within a glovebox. Between each set of reactions, silica gel, $\mathrm{SiO}_{\mathrm{x}}$ wafer, and CDO wafers were thoroughly washed with anhydrous toluene.

\subsubsection{Results on organic molecules}


a)

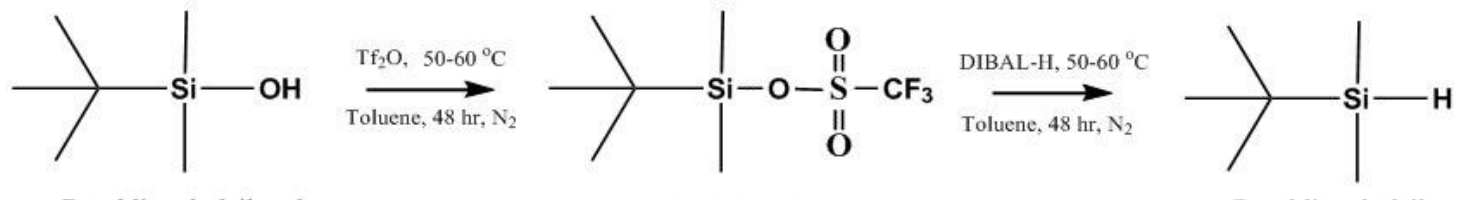

t-Butyldimethylsilanol

TBDMS Triflate

t-Butyldimethylsilane

b)

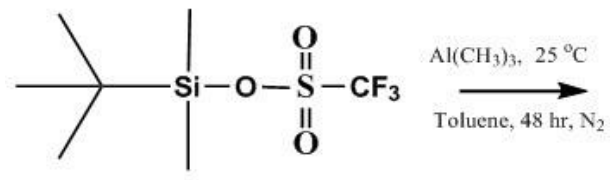

TBDMS Triflate

c)

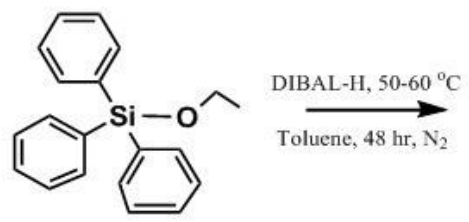

Ethoxytriphenylsilane

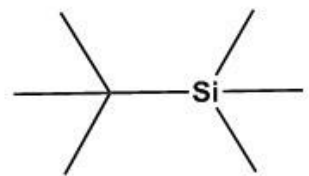

tert-Butyltrimethylsilane

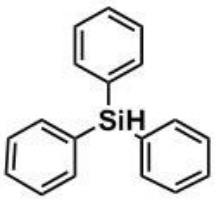

Triphenylsilane

Scheme 4.3. Schemes of a) Synthesis of TBDMS Triflate and tert-Butyldimethylsilane, b) Synthesis of tert-Butyltrimethylsilane c) Synthesis of Triphenylsilane.

\section{a) tert-Butyldimethylsilanol}

tert-Butyldimethylsilanol $\left(\left(\mathrm{CH}_{3}\right)_{3} \mathrm{CSi}\left(\mathrm{CH}_{3}\right)_{2} \mathrm{OH}\right)$ was used as a control molecule for the reaction scheme shown in Figure 4.3a. Reaction kinetics were studied by ${ }^{1} \mathrm{H}-\mathrm{NMR}$ spectroscopy and reaction products were confirmed by ${ }^{19}$ F-NMR, FTIR and Gas Chromatography-Mass Spectrometry (GC-MS). Transformation of the molecule to triflate and hydride molecules can be readily tracked by NMR, IR, and GC-MS techniques enabling optimization of the reaction conditions and yield. The kinetics of the triflation reaction was monitored by ${ }^{1} \mathrm{H}-\mathrm{NMR}$ of the reaction mixture over 72 hours, within 48 hours the reaction goes to completion (see Figure 4.3). The product of the reaction was further confirmed by parent ion peak at 265.0 g. $\mathrm{mol}^{-1}$ in the mass spectrum. 
In step 2, the reduction of TBDMS-OTf to tert-Butyldimethylsilane was achieved by treating the TBDMS-OTf with DIBAL-H reagent; the corresponding ${ }^{1} \mathrm{H}-\mathrm{NMR}$ spectrum was shown in Figure 4.4. After 24 hours of reaction, a new multiplet assigned to $\mathrm{Si}-\mathrm{H}$ at $\delta$ $3.64(\mathrm{~m}, 1 \mathrm{H})$ suggest the formation of hydrogen-terminated product, completes the reaction within 48 hours. In ${ }^{19}$ F-NMR spectra, shown in Figure 4.5, fluorine peak corresponding to triflate become negligible after 48 hours, confirm the reaction completion. The observed FTIR peak at $2105.7 \mathrm{~cm}^{-1}$ due to the formation of Si-H bond and a GC-MS parent ion peak at $116.1 \mathrm{~g} \cdot \mathrm{mol}^{-1}$ further confirmed the formation of the expected silane.

\section{Synthesis of tert-Butyldimethylsilyltrifluoromethanesulfonate (TBDMS-OTf): $1 \mathrm{mmol}$}

of tert-Butyldimethylsilanol and $2 \mathrm{mmol}$ of triflicanhydride reagents were dissolved in $1 \mathrm{ml}$ of anhydrous toluene solvent and mixed at 50 to $60{ }^{\circ} \mathrm{C}$ for up to 72 hours. Percentage yields were calculated from ${ }^{1} \mathrm{H}-\mathrm{NMR}$ of the crude reaction mixture, suggests a 30, 60, 98 and $100 \%$ product yield for $2,24,48$ and 72 hours respectively. ${ }^{1} \mathrm{H}-\mathrm{NMR}\left(\mathrm{C}_{6} \mathrm{D}_{6}, 400 \mathrm{MHz}\right) \delta$ $0.60(\mathrm{~s}, 9 \mathrm{H})$ and $\delta-0.00(\mathrm{~s}, 6 \mathrm{H}) ;{ }^{19} \mathrm{~F}-\mathrm{NMR}\left(\mathrm{CDCl}_{3}, 400 \mathrm{MHz}\right) \delta-76.84(\mathrm{~s}, 3 \mathrm{~F})$; GCMS calculated for $\mathrm{CF}_{3} \mathrm{SO}_{3} \mathrm{Si}\left(\mathrm{CH}_{3}\right)_{2} \mathrm{C}\left(\mathrm{CH}_{3}\right)_{3}$ 264.34, found 265.0; IR (KBr) v $v_{\max } 3027,2926$, $2857,1228,1193,1039 \mathrm{~cm}^{-1}$. 


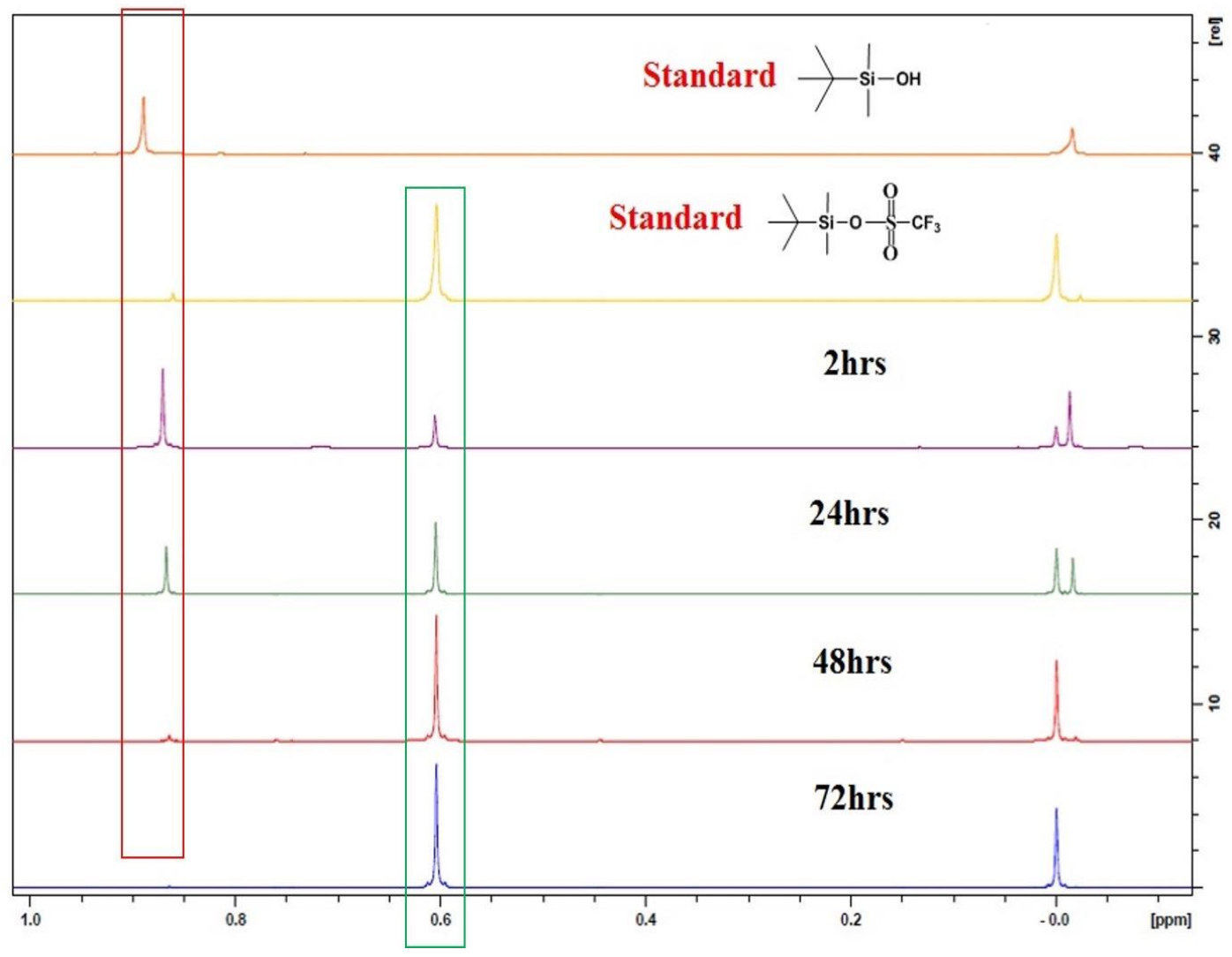

Figure 4.3. ${ }^{1} \mathrm{H}-\mathrm{NMR}\left(\mathrm{C}_{6} \mathrm{D}_{6}, 400 \mathrm{MHz}\right)$ reaction progress study of TBDMS Triflate synthesis at 2, 24,48 and 72 hours respectively. Increase in peak intensities at $\delta 0.60(\mathrm{~s}, 9 \mathrm{H})$ and $\delta-0.00(\mathrm{~s}, 6 \mathrm{H})$ corresponding to TBDMS Triflate can be observed.

Synthesis of tert-Butyldimethylsilane: $1 \mathrm{mmol}$ of tert-Butyldimethylsilyltrifluromethane sulfonate and $2 \mathrm{mmol}$ of DIBAL-H reagents were dissolved in $1 \mathrm{ml}$ of anhydrous toluene solvent and mixed at 50 to $60{ }^{\circ} \mathrm{C}$ for up to 72 hours. Percent yields were calculated from ${ }^{1} \mathrm{H}-\mathrm{NMR}$ of the crude reaction mixture, suggests $0,61,89$ and $91 \%$ product conversion after 2, 24, 48 and 72 hours respectively. ${ }^{1} \mathrm{H}-\mathrm{NMR}\left(\mathrm{CDCl}_{3}, 400 \mathrm{MHz}\right) \delta 3.64(\mathrm{~m}, 1 \mathrm{H}), \delta$ $0.88(\mathrm{~s}, 9 \mathrm{H}), \delta 0.02(\mathrm{~d}, J=3.1 \mathrm{~Hz}, 6 \mathrm{H})$; GCMS calculated for $\mathrm{HSi}\left(\mathrm{CH}_{3}\right)_{2} \mathrm{C}\left(\mathrm{CH}_{3}\right)_{3} 116.28$, found 116.10; IR (KBr) $v_{\max } 3027,2926,2857,2105 \mathrm{~cm}^{-1}$. 


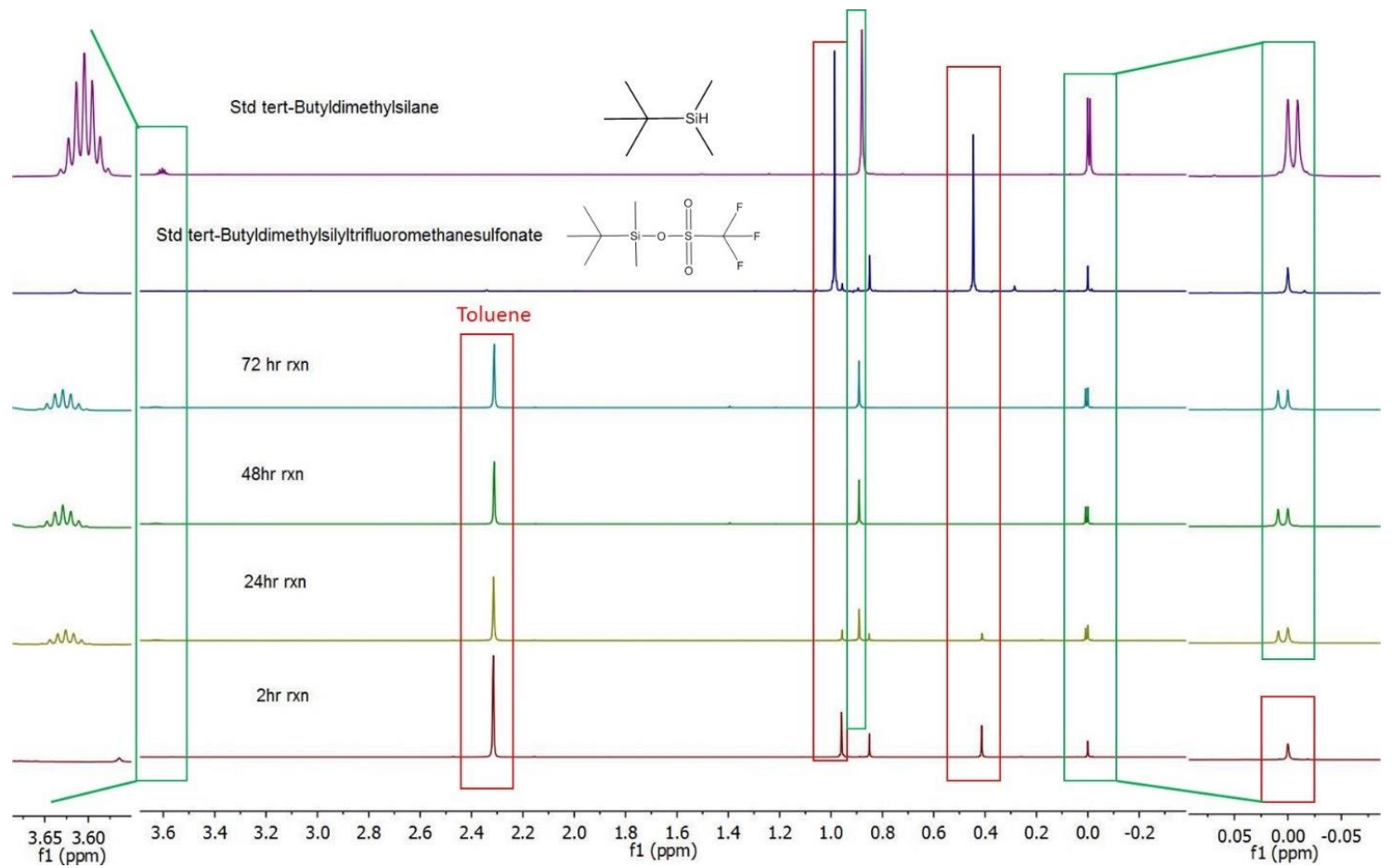

Figure 4.4. ${ }^{1} \mathrm{H}-\mathrm{NMR}\left(\mathrm{CDCl}_{3}, 400 \mathrm{MHz}\right)$ reaction progress study of tert-Butyldimethylsilane synthesis at 2, 24, 48 and 72 hours respectively. Peaks in the regions of $\delta 0.00$ and $\delta 3.64 \mathrm{ppm}$ were magnified and shown on the right and left-hand side of the original spectra. The appearance of multiplet at $3.64(\mathrm{~m}, 1 \mathrm{H})$ after 24 hours of reaction suggests the formation of the hydrogenterminated product. The decrease in the peak intensities of reactant and increase in peak intensities of the product can be observed here. 


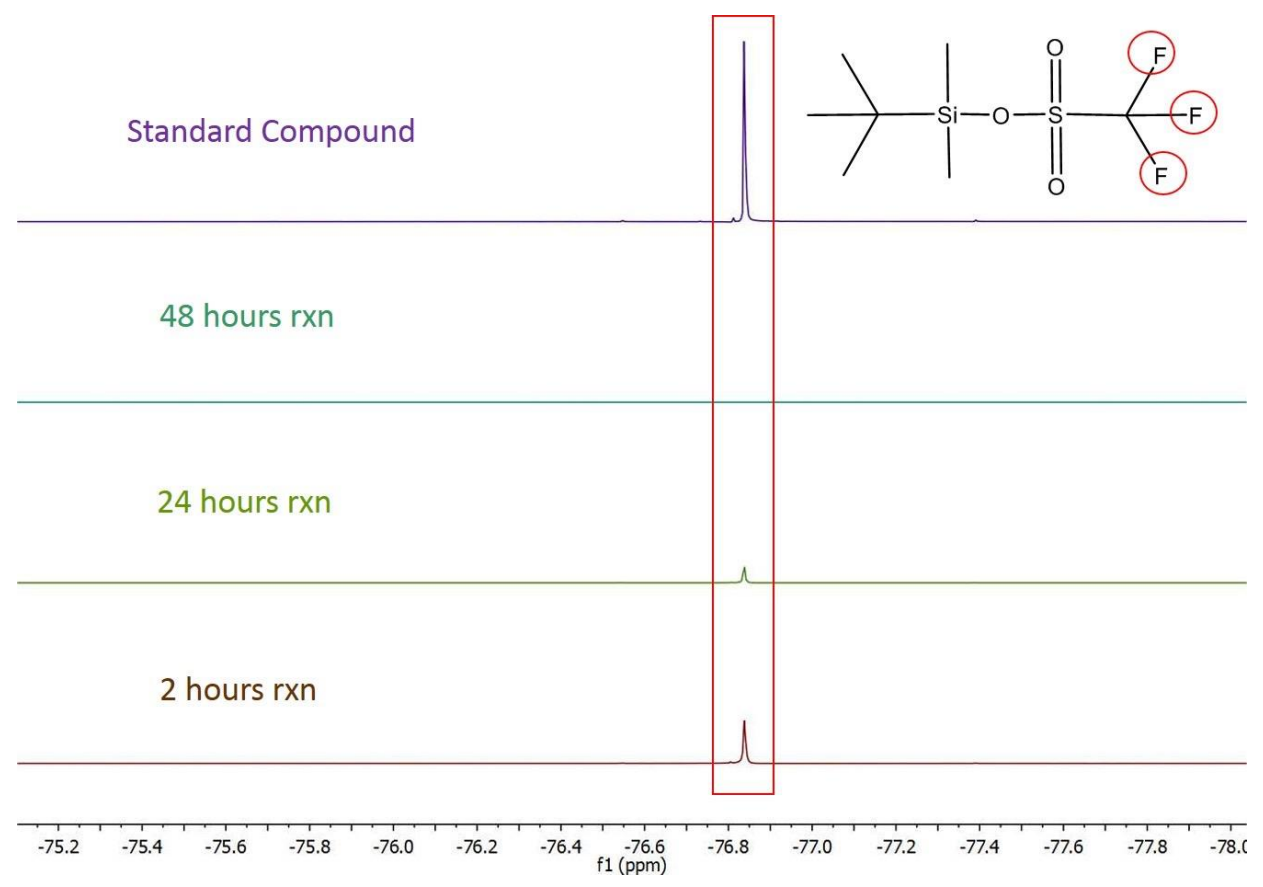

Figure 4.5. ${ }^{19} \mathrm{~F}-\mathrm{NMR}\left(\mathrm{CDCl}_{3}, 400 \mathrm{MHz}\right)$ reaction progress study of tert-Butyldimethylsilane synthesis at 2, 24 and 48 hours respectively. Fluorine peak intensity at $\delta-76.84 \mathrm{ppm}$ corresponding to the reactant TBDMS Triflate, decreases from 2 hours to 24 hours and becomes negligible after 48 hours of reaction can be observed.

\section{b) Synthesis of tert-Butyltrimethylsilane.}

It was possible to methyl terminate the TBDMS-OTf by treating it with Trimethylaluminum at $25{ }^{\circ} \mathrm{C}$ in 24 hours. In ${ }^{1} \mathrm{H}-\mathrm{NMR}$ spectra shown in Figure 4.6 , the presence of 1:1 ratio singlet peaks corresponding to methyl groups of tert-Butyl and silicon suggest the methyl termination.

Synthesis of tert-Butyltrimethylsilane: $1 \mathrm{mmol}$ of tert-Butyldimethyltrifluoro methane sulfonate and $2 \mathrm{mmol}$ of trimethylaluminum reagents were dissolved in anhydrous toluene solvent and mixed at $25{ }^{\circ} \mathrm{C}$ for 24 hours. ${ }^{1} \mathrm{H}-\mathrm{NMR}\left(\mathrm{C}_{6} \mathrm{D}_{6}, 400 \mathrm{MHz}\right) \delta 0.93(\mathrm{~s}, 9 \mathrm{H}), \delta 0.01$ (s, 9H); GCMS calculated for $\left(\mathrm{CH}_{3}\right)_{3} \mathrm{SiC}\left(\mathrm{CH}_{3}\right)_{3}$ 130.30, found 130.1. 


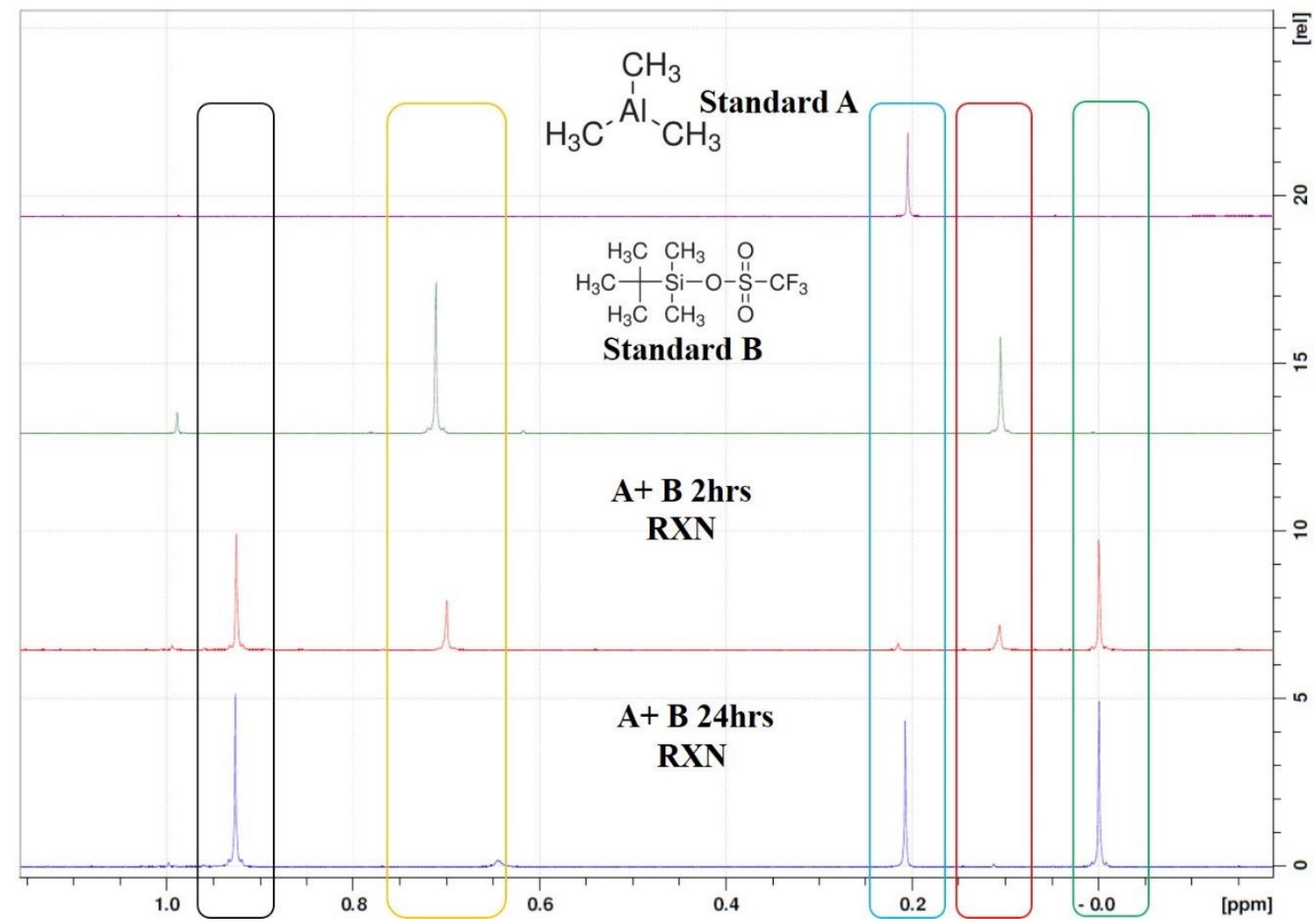

Figure 4.6. ${ }^{1} \mathrm{H}-\mathrm{NMR}\left(\mathrm{DMSO} \mathrm{d}_{6}\right)$ reaction progress study of tert-Butyltrimethylsilane synthesis at 2, 24 hours of reaction. A gradual increase in peak intensities at $\delta 0.93(\mathrm{~s}, 9 \mathrm{H}), \delta 0.01(\mathrm{~s}, 9 \mathrm{H})$ with 1:1 ratio corresponding to methyl groups of the product i.e., tert-butyl and 3 methyl groups on silicon can be observed.

Thus, DIBAL-H and Trimethylaluminum reagents successfully converted the triflate ester of silanol to hydride and methyl groups. In addition to tertButyldimethylsilanol, the hydrogen termination on Ethoxytriphenylsilanol was confirmed.

\section{c) Ethoxytriphenylsilane}

In addition to tert-Butyldimethylsilanol, the reaction pathway on Ethoxytriphenylsilane was tested as shown in Scheme 4.3c. ${ }^{1} \mathrm{H}-\mathrm{NMR}$ data of the expected silane suggest the hydrogen termination of silicon by DIBAL-H reduction (see Figure 4.7). 
Synthesis of Triphenylsilane: $1 \mathrm{mmol}$ of ethoxytriphenylsilane and $2 \mathrm{mmol}$ of DIBAL-H reagent were dissolved in anhydrous toluene solvent and mixed at $50-60{ }^{\circ} \mathrm{C}$ for 48 hours. Percentage yield calculated from ${ }^{1} \mathrm{H}-\mathrm{NMR}$ of the crude reaction mixture was $48 \% .{ }^{1} \mathrm{H}$ NMR (DMSO-d $6,400 \mathrm{MHz}) \delta 7.58(\mathrm{~m}, 15 \mathrm{H}), \delta 5.7(\mathrm{~s}, 1 \mathrm{H}) ; \mathrm{IR}(\mathrm{KBr}) v_{\max } 3067,2119$, $1427,1113,802,730,697 \mathrm{~cm}^{-1}$.

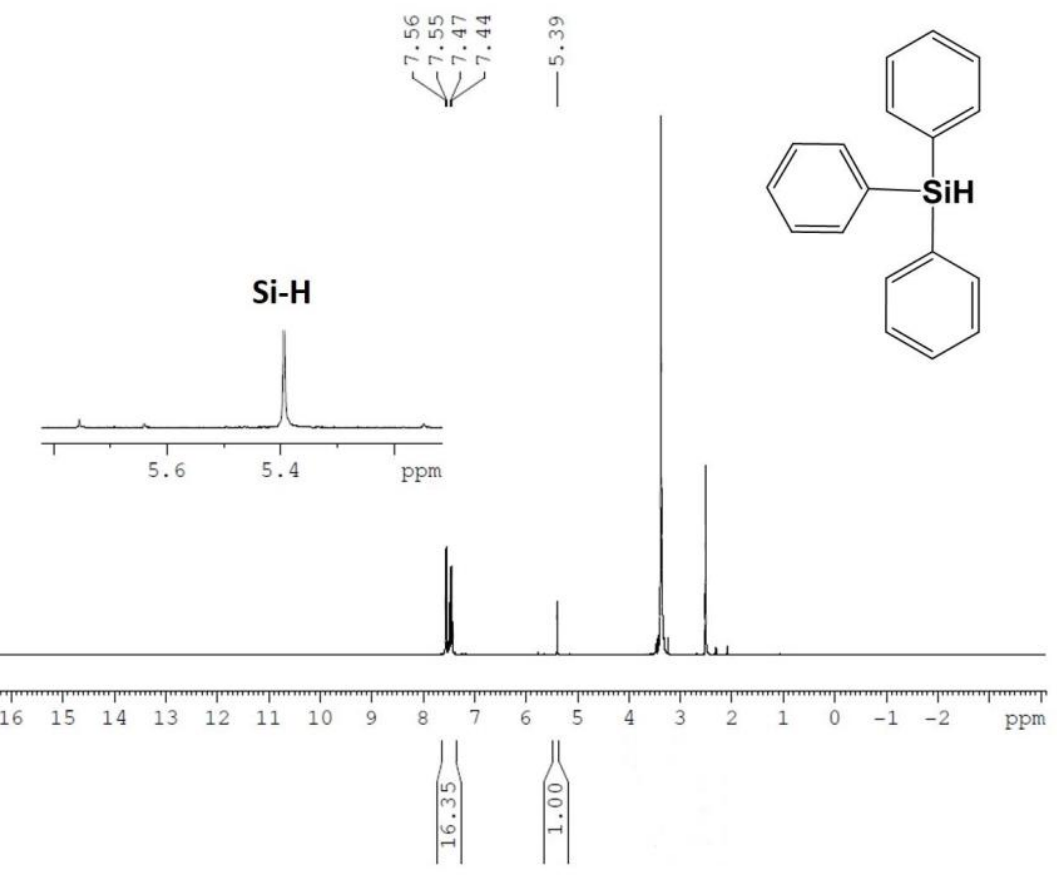

Figure 4.7. ${ }^{1} \mathrm{H}-\mathrm{NMR}\left(\mathrm{DMSO}-\mathrm{D}_{6}\right)$ of triphenylsilane. Peaks at $\delta 5.39(\mathrm{~s}, 1 \mathrm{H}), \delta 7.5(\mathrm{~m}, 15 \mathrm{H})$ were assigned to hydrogen-terminated silicon $(\mathrm{Si}-\mathrm{H})$ and protons of phenyl groups respectively. Peaks at $\delta 2.3(\mathrm{~S}, 1 \mathrm{H})$ and $\delta 3.52(\mathrm{~s}, 9 \mathrm{H})$ corresponding to toluene solvent and DIBAL-H reagent.

\subsubsection{Results on Silica gel reaction}

The reaction scheme discussed above can be readily adapted for modification of silica gel used in classic chromatography, pharmaceutical, cosmetic and in many other applications involving silanol group. In recent years, silica aerogel has been widely used in a variety of industries due to its exceptional properties such as low thermal conductivity, 
high porosity, high optical transmission, high specific surface area, low dielectric constant, low refractive index and low sound velocity; however its moisture sensitivity has limited its practical use $\mathrm{e}^{93,94}$.

In the literature, various derivatization strategies have been studied to alter the hydrophobicity of silica gel; these include methyltrimethoxysilane (MTMS), hexamethyldisilazane (HMDZ), dimethylchlorosilane (DMCS), trimethylethoxysilane (TMES), and hexadecyltrimethoxysilane (HDTMS) ${ }^{95}$. These prevailing methods render the surface hydrophobic by replacing hydrogen from hydroxyl $(\mathrm{SiO}-\mathrm{H})$ functional group ${ }^{91}$, 96-104 which leads to the formation of siloxy groups (SiO-R). But the method developed here leads to the selective replacement of hydroxyl (-OH) functional groups with a nonpolar $\mathrm{Si}-\mathrm{C}$ group bearing long alkyl chains $\left(\mathrm{Si}-\mathrm{R}, \mathrm{R}=\mathrm{C}_{18} \mathrm{H}_{37}\right.$ or other long chain hydrocarbon) which resulted in less polar and a highly hydrophobic surface with lower dielectric constant.

Having established the feasibility of the reaction scheme on molecular species, its potential for derivatizing silica gel was explored. Silica gel, structurally similar to CDO. with its highly porous structure and a large number of air exposed hydroxyl functional groups provided a useful control to test the reaction scheme on a heterogeneous substrate ${ }^{105}$. 


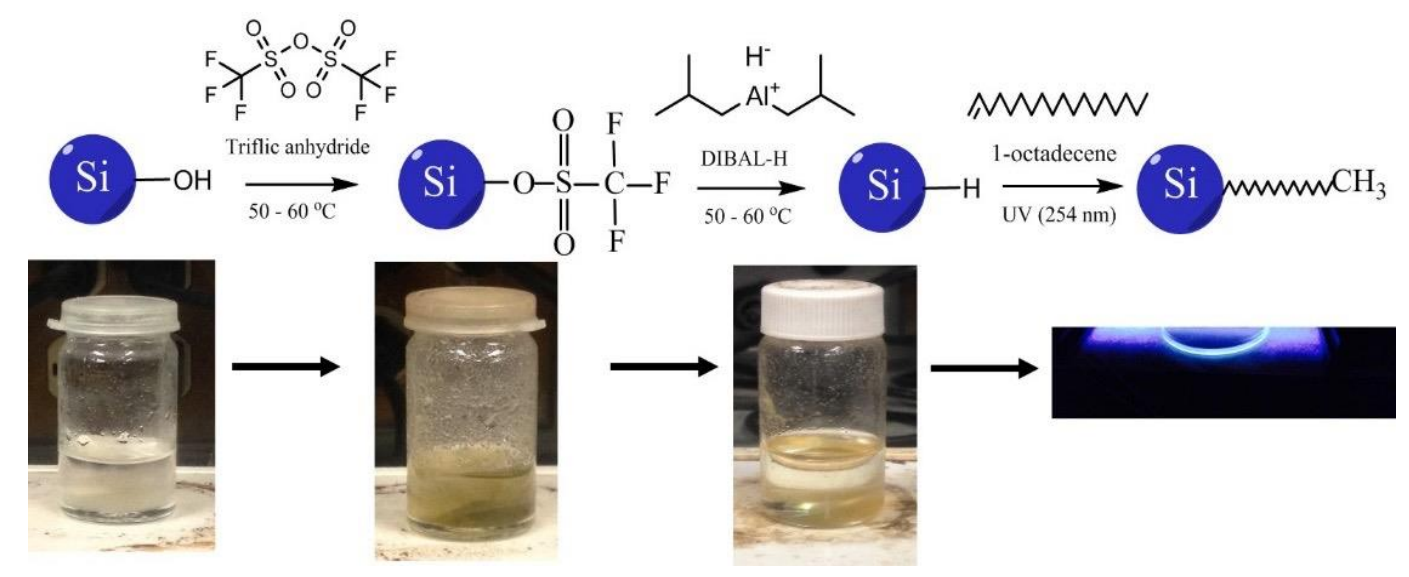

Figure 4.8. Experimental setup of silica gel reaction. After triflation reaction silica gel turned to black and after DIBAL-H reaction turned to golden brown color.

The experiments used chromatography-grade silica gel, with particles ranging from 75 to $250 \mu \mathrm{m}, 1.15 \times 10^{-6} \mathrm{~m}^{3} / \mathrm{g}$ specific volume, and surface area of $300 \mathrm{~m}^{2} / \mathrm{g}$, with a pore size of approximately $15 \mathrm{~nm}$. Before derivatization, the silica gel was dried by heating at $400 \mathrm{o}$ C for 4 hours and allowed to cool under modest vacuum (100 mTorr). Silica gel was then immersed in the reaction solution as shown in Figure 4.8. Reaction progress on the silica gel was studied by FTIR spectroscopy as shown in Figure 4.9. A broad peak at 3439 $\mathrm{cm}^{-1}$ was ascribed to the superposition of stretching vibrational modes of physically adsorbed water molecules and silicon hydroxyl (Si-OH) functional group. Peaks at 1626 $\mathrm{cm}^{-1}, 1480 \mathrm{~cm}^{-1}$, were assigned to the bending vibration of the water molecule and silanol respectively. $1102 \mathrm{~cm}^{-1}$ and $969 \mathrm{~cm}^{-1}$ peaks reflected the Si-O-Si stretch vibration modes of silica gel ${ }^{91,96,98,101-103}$. Triflation reaction with triflic anhydride resulted in new peaks (=Si-O-Tf) at $1033 \mathrm{~cm}^{-1}$, and $1257 \mathrm{~cm}^{-1}$ corresponding to $\mathrm{S}=\mathrm{O}$ asymmetric, symmetric stretches and an $1177 \mathrm{~cm}^{-1}$ peak ascribed to C-F stretching corresponding to formation of $\left(\equiv \mathrm{Si}-\mathrm{O}-\left(\mathrm{SO}_{2}\right) \mathrm{CF}_{3}\right)$ groups respectively. It should be noted that the trifilation reaction did 
not result in complete removal of the broad $\mathrm{OH}$ peak since the silica gel has $\mathrm{OH}$ groups not accessible to the chemical reagent.

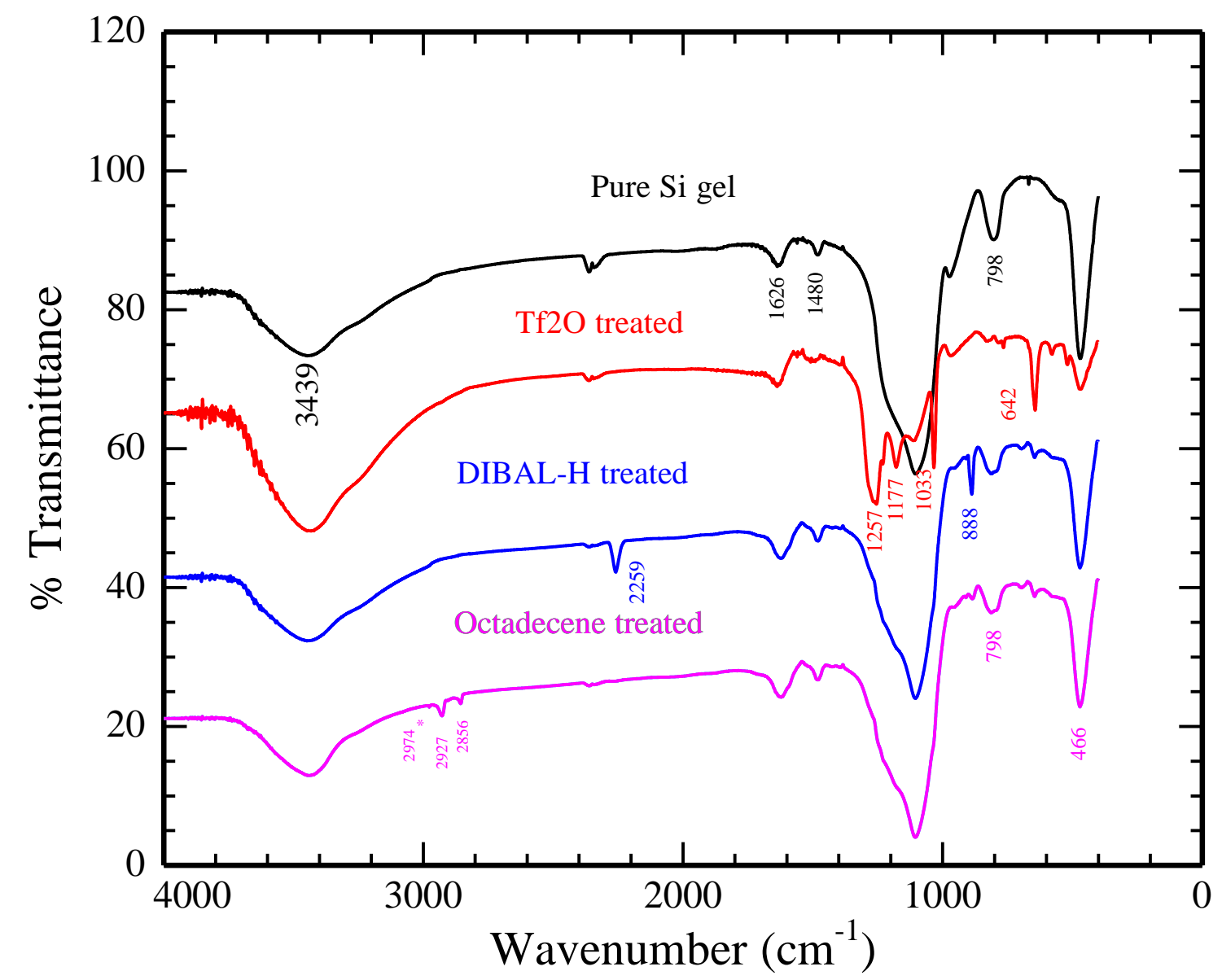

Figure 4.9. FTIR spectra of silica gel in various stages of the reaction scheme. Pure Si gel (black), after treatment with triflic anhydride (blue), DIBAL-H reduced (pink) \& after 1-octadecene treatment (red).

Further attempts to estimate the percent conversion of the surface silicon hydroxyl to Silyltrifluoromethanesulfonate ester were conducted by ${ }^{19} \mathrm{~F}$ NMR spectroscopy, based on the reaction between $\mathrm{Si}-\mathrm{OH}$ on the surface of silica particles and triflic anhydride (See equation-1). 


$$
\equiv \mathrm{Si}-\mathrm{OH}+\left(\mathrm{CF}_{3} \mathrm{SO}_{2}\right)_{2} \mathrm{O} \longrightarrow \equiv \mathrm{Si}-\mathrm{O}-\mathrm{SO}_{2}-\mathrm{CF}_{3}+\mathrm{CF}_{3}-\mathrm{SO}_{2}-\mathrm{OH}
$$

The chemical shifts of methyl fluorine in triflic anhydride (reactant) and triflic acid (product) occur at $-72.6 \mathrm{ppm}$ and $-77.3 \mathrm{ppm}$, respectively. Their integrated peak intensities allowed determination of the extent of the triflation reaction, assuming that the only source of triflic acid was from silanol esterification. However, if moisture is present, it can also hydrolyze the triflic anhydride to give two moles of triflic acid as shown in the following equation.

\section{$\left(\mathrm{CF}_{3} \mathrm{SO}_{2}\right)_{2} \mathrm{O}+\mathrm{H}_{2} \mathrm{O} \longrightarrow 2 \mathrm{CF}_{3} \mathrm{SO}_{2}-\mathrm{OH}$}

Therefore, a control experiment was carried out in a glovebox to examine the effect of moisture on measurement accuracy. By stirring the triflic anhydride in anhydrous toluene solvent for 24 and 48 hours at $60{ }^{\circ} \mathrm{C},{ }^{19} \mathrm{~F}-\mathrm{NMR}$ data showed that the peak integration ratio of triflic acid to triflic anhydride was 1:148 (or 1:296 wt. mole ratio) and 1:80 (or 1:160 wt. mole ratio), respectively. This indicated that moisture present in glovebox during triflation reaction was negligible. After 24 and 48 hours of triflation reaction at $60{ }^{\circ} \mathrm{C}$ with $0.2 \mathrm{~g}$ of silica gel, this ratio increased to $1: 9$ and $1: 2$, respectively (see Figure 4.10). Based on this, estimated conversion percentage in esterification reaction was $44 \%$ after 24 hours and then reached 100\% after 48 hours (by assuming a surface silanol concentration of $14 \mathrm{mmol} / 5 \mathrm{~g}$ silica gel with 4.6 groups $\mathrm{SiOH}$ per $\left.\mathrm{nm}^{2}\right)^{106}$. 


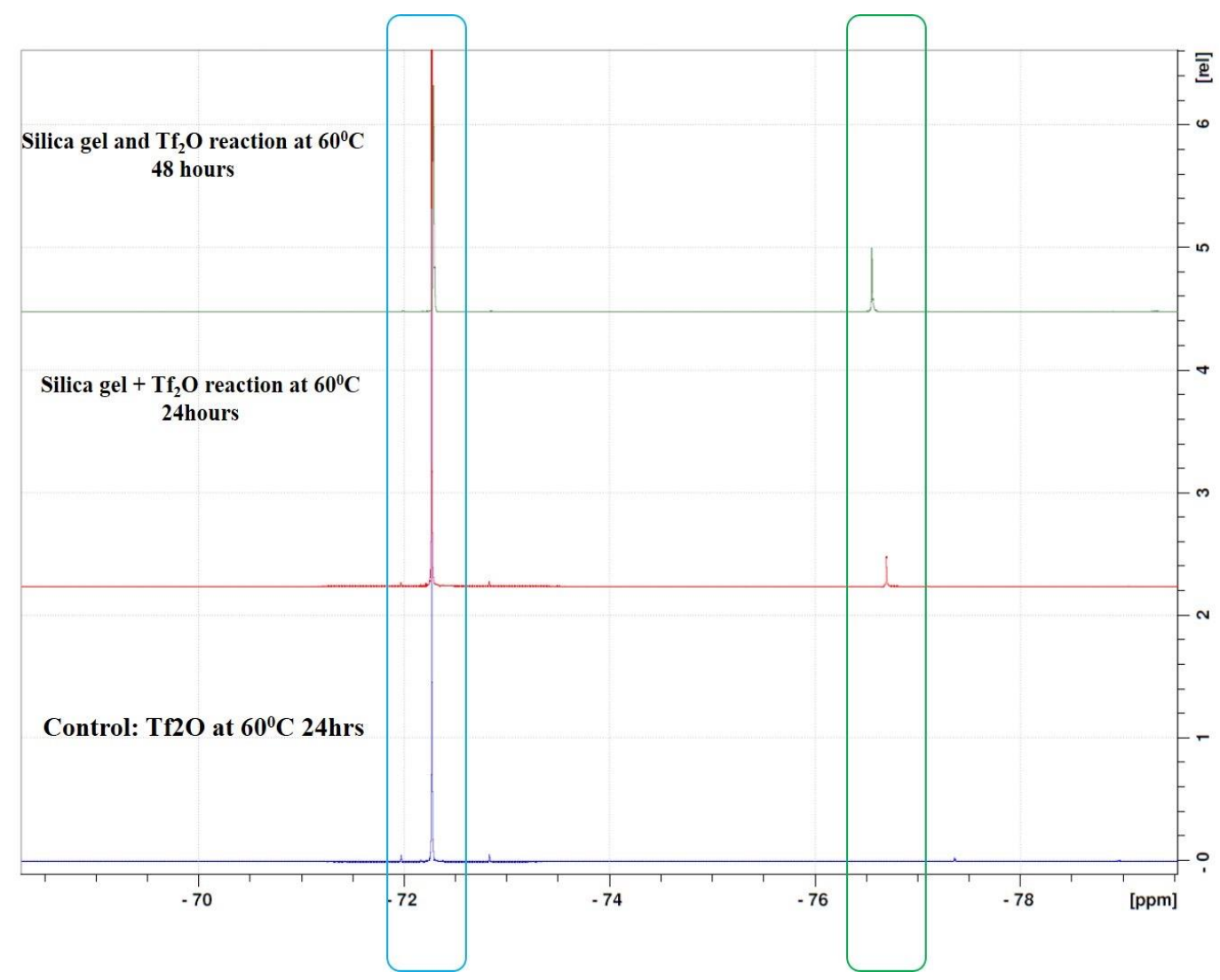

Figure 4.10. ${ }^{19} \mathrm{~F}$ NMR study of silica gel reaction with triflic anhydride. In a control experiment, a strong peak at $-72.6 \mathrm{ppm}$ corresponding to fluorine of triflic anhydride can be observed. In a reaction with silica gel peak intensity at $-77.3 \mathrm{ppm}$ corresponding to triflic acid increases and reaches to maximum after 48 hours of reaction.

After reduction with DIBAL-H reagent, new peaks in FT-IR appeared at $2259 \mathrm{~cm}^{-1}$ and $888 \mathrm{~cm}^{-1}$, which correspond to $\mathrm{Si}-\mathrm{H}$ stretching, and bending vibrations ${ }^{96}$; see the pink curve in Figure 4.9. Note that $\mathrm{Si}-\mathrm{H}$ frequency depends on the polarity of the other three bonds with silicon, varying from 2109 to 2250 in going from C-Si-H to O-Si-H ${ }^{107}$.

Silicon hydride groups $(\mathrm{Si}-\mathrm{H})$ were further modified to alkylated silica $(\mathrm{Si}-\mathrm{R})$ by treating with 1 -octadecene in the presence of UV light $(254 \mathrm{~nm})$ for 2 hours ${ }^{108}$. Samples were washed with anhydrous chloroform $\left(\mathrm{CHCl}_{3}\right)$ solvent to remove excess adsorbed 1- 
octadecene and then air dried ${ }^{108,109}$. New peaks were observed at $2856 \mathrm{~cm}^{-1}$ and $2927 \mathrm{~cm}^{-1}$ corresponding to $-\mathrm{CH}_{2}$ symmetric and asymmetric stretches, and shoulder peak at $2974 \mathrm{~cm}^{-}$ ${ }^{1}$ corresponding to $-\mathrm{CH}_{3}$, confirming the alkyl chain derivatization of silica gel surface ${ }^{91}$, 98, 99, 101-103.

In FTIR of alkyl derivatized silica gel spectrum, the absence of peaks corresponding to alkene $\mathrm{C}-\mathrm{H}$ stretch $(\mathrm{C}=\mathrm{C}-\mathrm{H})$ at $3083 \mathrm{~cm}^{-1}$ and $\mathrm{C}=\mathrm{C}$ stretch at $1644 \mathrm{~cm}^{-1}$ indicated the absence of 1-Octadecene molecules physically adsorbed on the silica gel surfaces. To further gauge the effect of adsorbed alkenes, as opposed to chemically bonded alkyl groups, a control experiment was undertaken. The experiment involved, immersing the dried silica gel in a 1-octadecene solvent and exposing it to UV light under similar conditions, and finally washed with chloroform solvent after the completion of the reaction. The FTIR spectrum (see Figure 4.11) of silica gel after these steps showed no significant peaks corresponding to alkyl chains, indicating negligible adsorption of 1-octadecene on the silica surface. 


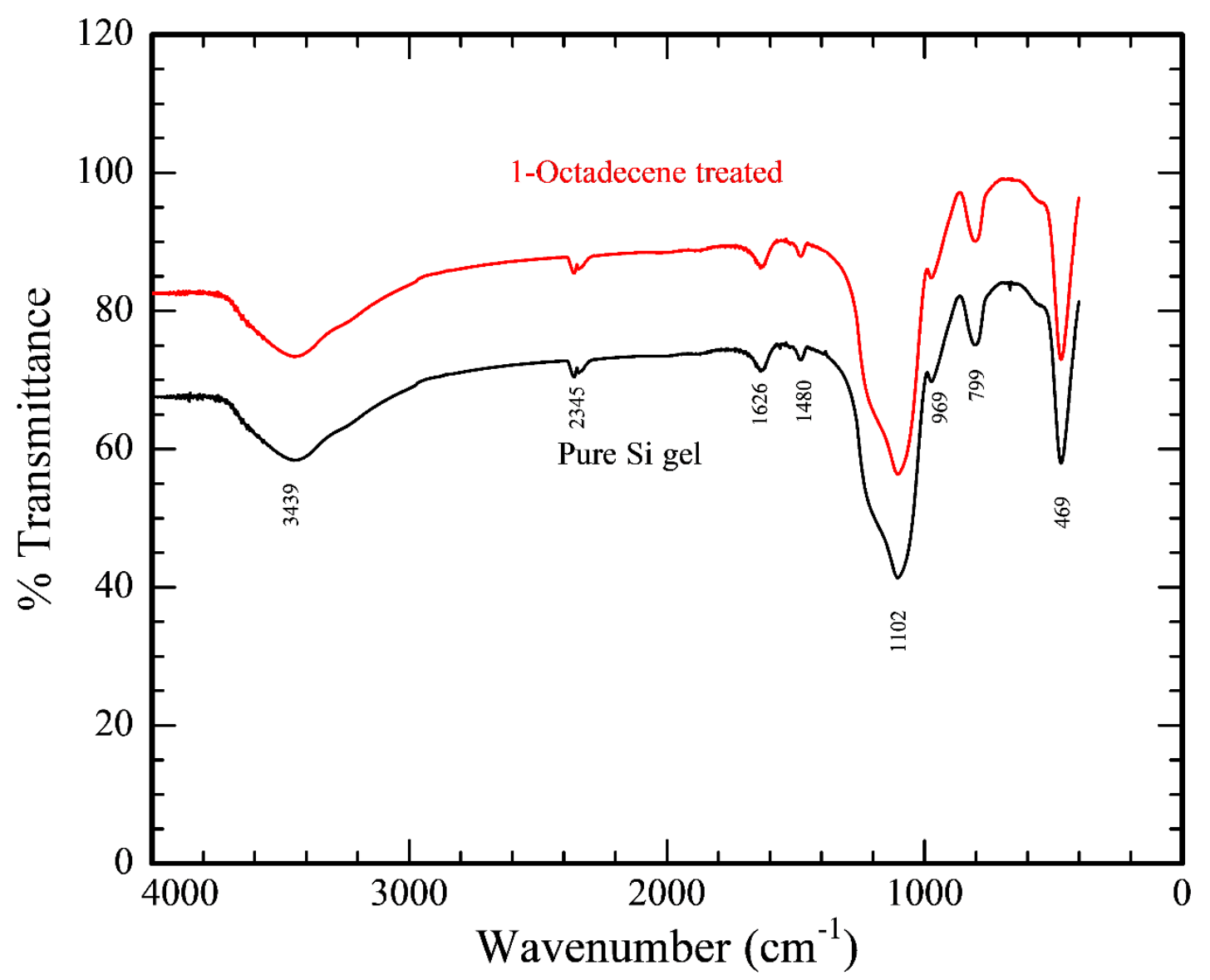

Figure 4.11. FTIR of silica gel control experiment. Comparison of pure silica gel before reaction and after treatment with 1-Octadecene in the presence of UV light. No peaks corresponding to alkyl chains can be observed in 2800 to $3000 \mathrm{~cm}^{-1}$ region.

After studying the reaction on organic molecules and heterogeneous silica gel, the method was tested on a classic $\mathrm{SiO}_{\mathrm{x}}$ wafer. Reaction progress was studied by FTIR and water contact angle (CA) measurement.

\subsubsection{Results on $\mathrm{SiOx}$ wafer reaction}

Derivatization studies of $\mathrm{SiO}_{\mathrm{x}}$ coated wafer provided a suitable comparison with silica gel (vide supra) and low $k \mathrm{CDO}$ (vide infra). These studies utilized silicon wafers decorated 
with $100 \mathrm{~nm}$ thick silicon dioxide treated in identical reaction conditions. A clean $\mathrm{SiO}_{\mathrm{x}}$ wafer before the reaction showed the peaks (the black trace in Figure 4.12) corresponding to the stretching $\left(3376 \mathrm{~cm}^{-1}\right)$ and bending vibrations $\left(1296 \mathrm{~cm}^{-1}\right)$ of the silanol (Si-OH); the symmetric $\left(890 \mathrm{~cm}^{-1}\right)$ and asymmetric stretching $\left(1105 \mathrm{~cm}^{-1}\right)$ of cage Si-O-Si; and the bending vibration $\left(610 \mathrm{~cm}^{-1}\right)$ of $\mathrm{Si}-\mathrm{O}-\mathrm{Si}^{82}$. Lower peak intensities associated with $\mathrm{O}-\mathrm{H}$ stretch vibrations (at $3500 \mathrm{~cm}^{-1}$ ) when compared to Si-O-Si peaks (at $1100 \mathrm{~cm}^{-1}$ ), indicated a lower net content of surface $\mathrm{Si}-\mathrm{OH}$ groups on $\mathrm{SiO}_{\mathrm{x}}$ wafer films than silica gel (cf. black curves in Figures 4.9 and 4.12).

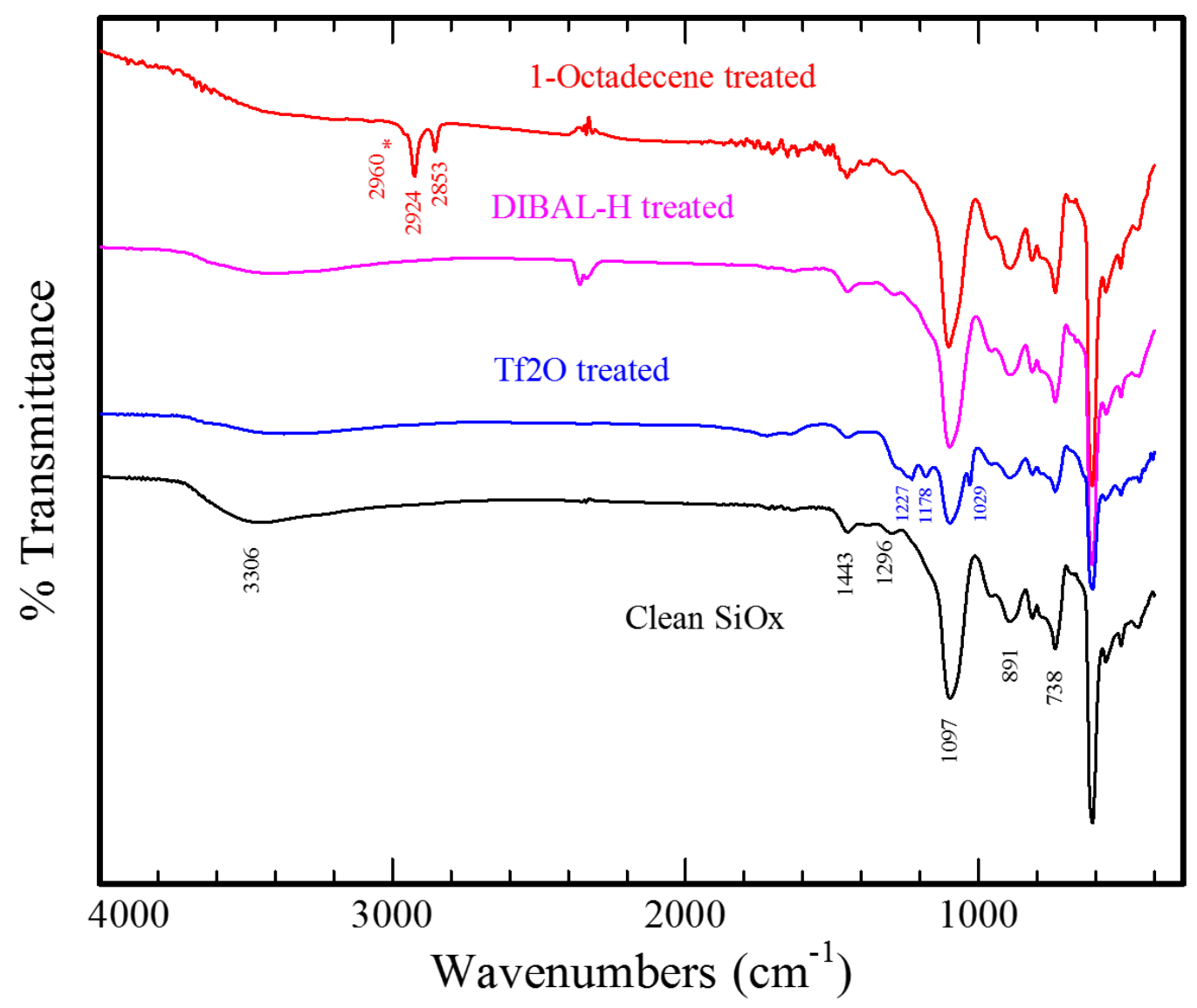

Figure 4.12. FT-IR spectra of $\mathrm{SiO}_{\mathrm{x}}$ wafer at various stages of the reaction: clean $\mathrm{SiO}_{\mathrm{x}}$ film (black); after treatment with triflic anhydride (blue); DIBAL-H reduced (pink); and after treatment with 1 -octadecene (red). 
The Si-OH absorption peak intensity significantly reduced upon triflation consistent with the surface accessibility of most $\mathrm{Si}-\mathrm{OH}$ groups in the nonporous $\mathrm{SiO}_{\mathrm{x}}$ film(cf. black and blue traces). After the triflation reaction, new peaks appeared at $1039,1228 \mathrm{~cm}^{-1}$ and $1193 \mathrm{~cm}^{-1}$ corresponding to symmetric and asymmetric stretches of $\mathrm{S}=\mathrm{O}$ bond, and $\mathrm{C}-\mathrm{F}$ stretch, respectively, as in the case of silica gel. However, their relative peak intensities were proportionately smaller. After DIBAL-H reduction, the $\mathrm{Si}-\mathrm{H}$ peak at $2259 \mathrm{~cm}^{-1}$ was not observed presumably because of the lower total content of $\mathrm{SiOH}$ groups on the $\mathrm{SiO}_{\mathrm{x}}$ film surface compared to silica gel (cf. pink curves in Figures 4.9 and 4.12). Nevertheless, silica gel Si-H peaks disappeared upon exposure to air within half an hour. Similarly, limited stability of the $\mathrm{Si}-\mathrm{H}$ groups in the air has been previously noted when the remaining three group bound to silicon are highly polar, such as $\mathrm{Si}-\mathrm{O}$.

Hydrosilylation of the hydrogen-terminated Silicon surface with long-chain terminal alkene was conducted without exposing the sample to atmospheric moisture. After the photochemical reaction, the presence of peaks at 2853 and $2924 \mathrm{~cm}-1$, corresponding to $\mathrm{CH} 2$ symmetric and asymmetric stretches, and a shoulder peak at $2976 \mathrm{~cm}-1$ (red curve and red inset in Figure 3) confirmed the hydrosilylation. The ratio of C-H (2800-2950 cm1) to $\mathrm{Si}-\mathrm{O}-\mathrm{Si}(1100 \mathrm{~cm}-1)$ peak intensities was higher than silica gel (cf. Figures 4.9 and 4.12) and could arise from a greater percentage of inaccessible surface $\mathrm{SiOH}$ groups in silica gel. The absence of peaks at 3083 and $1004 \mathrm{~cm}-1$ corresponding to alkene $\mathrm{C}=\mathrm{C}-\mathrm{H}$ stretching and bending vibrations, and the $\mathrm{C}=\mathrm{C}$ stretch at $1644 \mathrm{~cm}-1$ of 1 -octadecene, suggested that 1-octadecene was chemically bonded to the silicon surface rather than 
physically adsorbed. Contact angles before and after derivatization of $\mathrm{SiO}_{\mathrm{x}}$ surfaces were $11 \pm 2^{\circ}$ and $101 \pm 4^{\circ}$ respectively.

\subsubsection{Results on CDO wafer reaction}

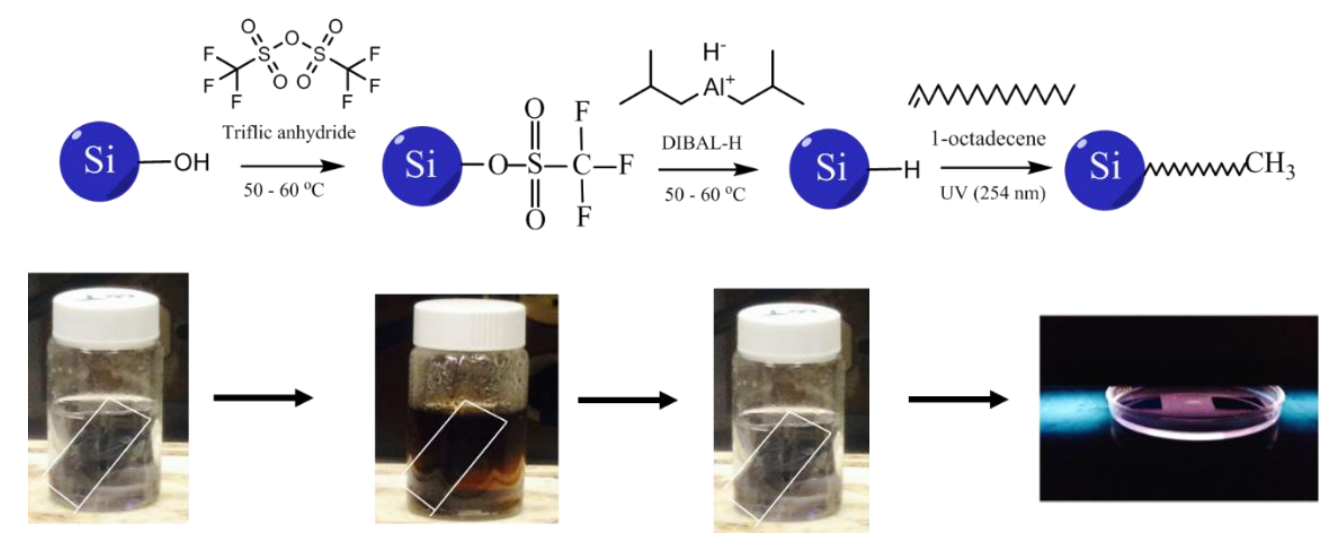

Figure 4.13. Common reaction pathway for hydrogen termination, Octadecane derivatization and experimental setup of carbon doped silicon oxide (CDO) wafer.

FT-IR, X-ray photoelectron spectroscopy (XPS) and water contact angles (CA) techniques were employed to monitor the progress of the reaction, and the appearance of new functional groups on $\mathrm{CDO}$ wafer surfaces. When compared to the $\mathrm{SiO}_{\mathrm{x}}$ wafer the water contact angle on CDO after RCA clean, was significantly higher $\left(36 \pm 3^{\circ}\right.$ vs. $11 \pm 2^{\circ}$, see Table 4.2, below) consistent with a more hydrophobic surface due to its higher carbon content and a lower density of $\mathrm{SiOH}$ group on the top surface (cf. black curves in Figures 4.12 and 4.14).

Figure 4.14 shows the transmission FT-IR spectra of a CDO wafer at different stages of derivatization. Major peaks observed correlated well with the $\mathrm{SiO}_{\mathrm{x}}$ wafer IR peaks illustrated in Figure 4.12. However, significantly lower peak intensity in the $\mathrm{OH}$ vibrational frequency range $\left(\approx 3300 \mathrm{~cm}^{-1}\right)$ compared to silica gel or $\mathrm{SiO}_{\mathrm{x}}$ wafer (Figures 2,3$)$, implied a lower net content of $\mathrm{OH}$ on the $\mathrm{CDO}$ surface. Accordingly, after triflation, intensities of 
peaks associated with $\mathrm{S}=\mathrm{O}$ and $\mathrm{C}-\mathrm{F}$ stretches, (shown Figure 4.14, the blue curve (inset)), were smaller than the corresponding peaks observed in the triflated $\mathrm{SiO}_{\mathrm{x}}$ films (blue curve, Figure 4.12).

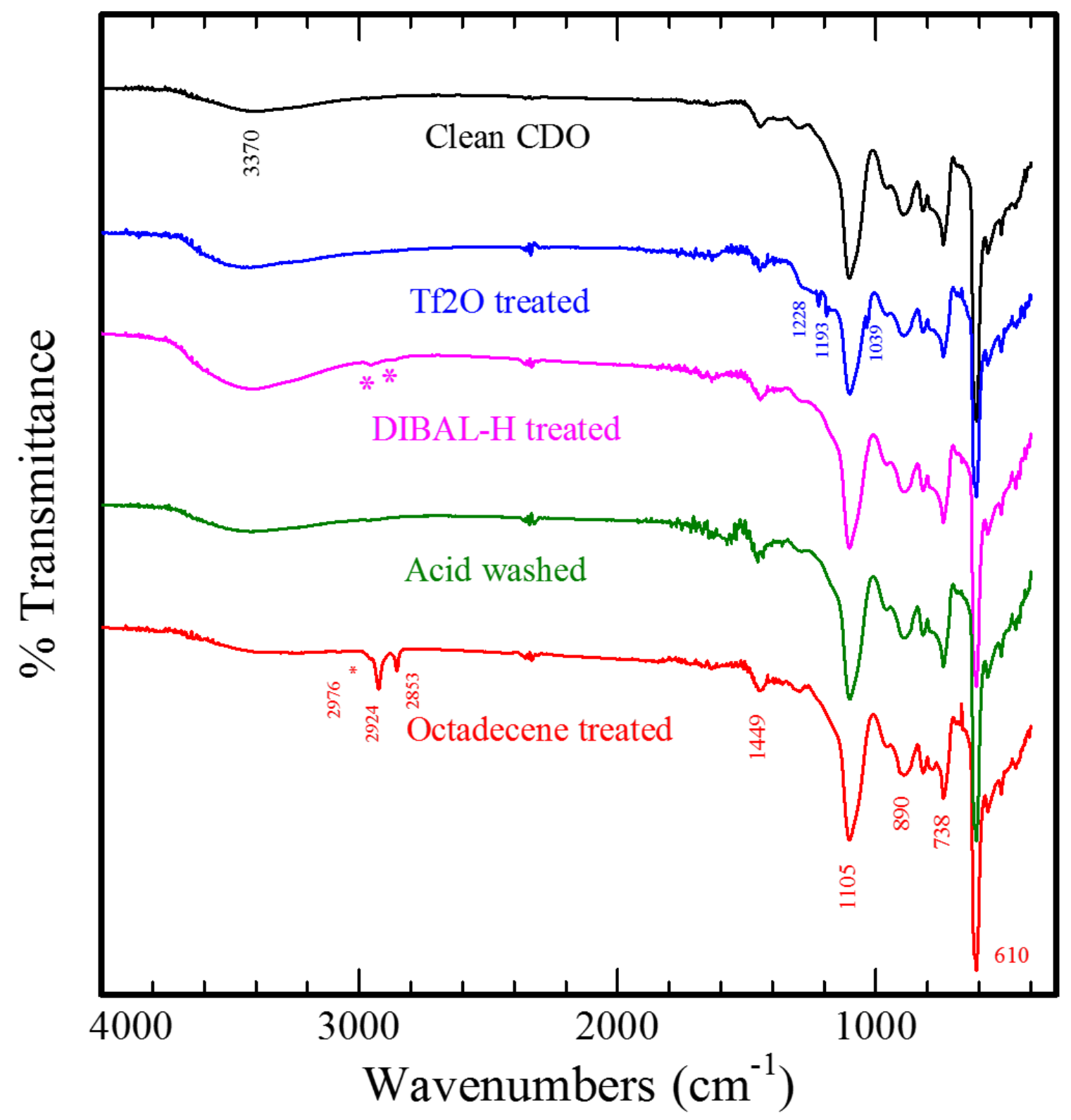

Figure 4.14. FT-IR spectra of $\mathrm{CDO}$ wafer in various stages of reaction; Clean CDO (black), after reaction with triflic anhydride (blue), after reaction with DIBAL-H (pink), after glacial acetic acid washing (green) and after octadecene derivatization (red). Stars indicated in the DIBAL-H treated spectrum correspond to alkyl groups arising from the surface-bound, excess DIBAL-H reaction 
products. Insets expand selected spectral regions of triflic anhydride $\mathrm{Tf}_{2} \mathrm{O}$ and octadecenederivatized silica in the IR spectra.

Reduction of triflate ester on the CDO surface using DIBAL-H proceeded as in the case of $\mathrm{SiO}_{\mathrm{x}}{ }^{110}$. After reduction with DIBAL-H several new peaks, not observed in silica gel or $\mathrm{SiO}_{x}$ surfaces, appeared in the range from $2800-3000 \mathrm{~cm}^{-1}$, attributed to alkyl C-H vibrations. These peaks could originate from traces of the degraded DIBAL-H reagent (e.g., diisobutyl groups), physically adsorbed or trapped in pores of CDO surface (pink trace, Figure 4.14). Also, supporting the above hypothesis was an observed increase in the peak intensity near the $\mathrm{OH}$ region $\left(3300 \mathrm{~cm}^{-1}\right)$ due to the formation of $\mathrm{Al}-\mathrm{OH}$ groups, a byproduct of the reduction reaction. Since an aluminum oxide or its hydroxide dissolves readily in an acidic medium, we treated the wafer with $25 \%$ glacial acetic acid in anhydrous toluene for five minutes to remove trapped/ degraded DIBAL-H reagent from the CDO surface. The FT-IR spectra collected after the acid treatment displayed a reduction in $\mathrm{OH}$ peak intensity and disappearance of alkyl shoulder-peaks (the green trace, Figure 4.14).

A control experiment to further test the hypothesis of DIBAL-H binding on the CDO surface entailed treating clean $\mathrm{CDO}, \mathrm{SiO}_{\mathrm{x}}$ wafers, and silica gel sample with a DIBAL-H reagent (see Figure 4.15) in anhydrous toluene. After 24 hours, samples were washed with dry toluene and dried. Their FT-IR spectra revealed the alkyl peaks only for the CDO confirming preferential DIBAL-H trapping/adsorption on the surface/pores of a slightly hydrophobic CDO surface (vide supra) as opposed to hydrophilic surfaces of silica gel or $\mathrm{SiO}_{x}$ wafer. 

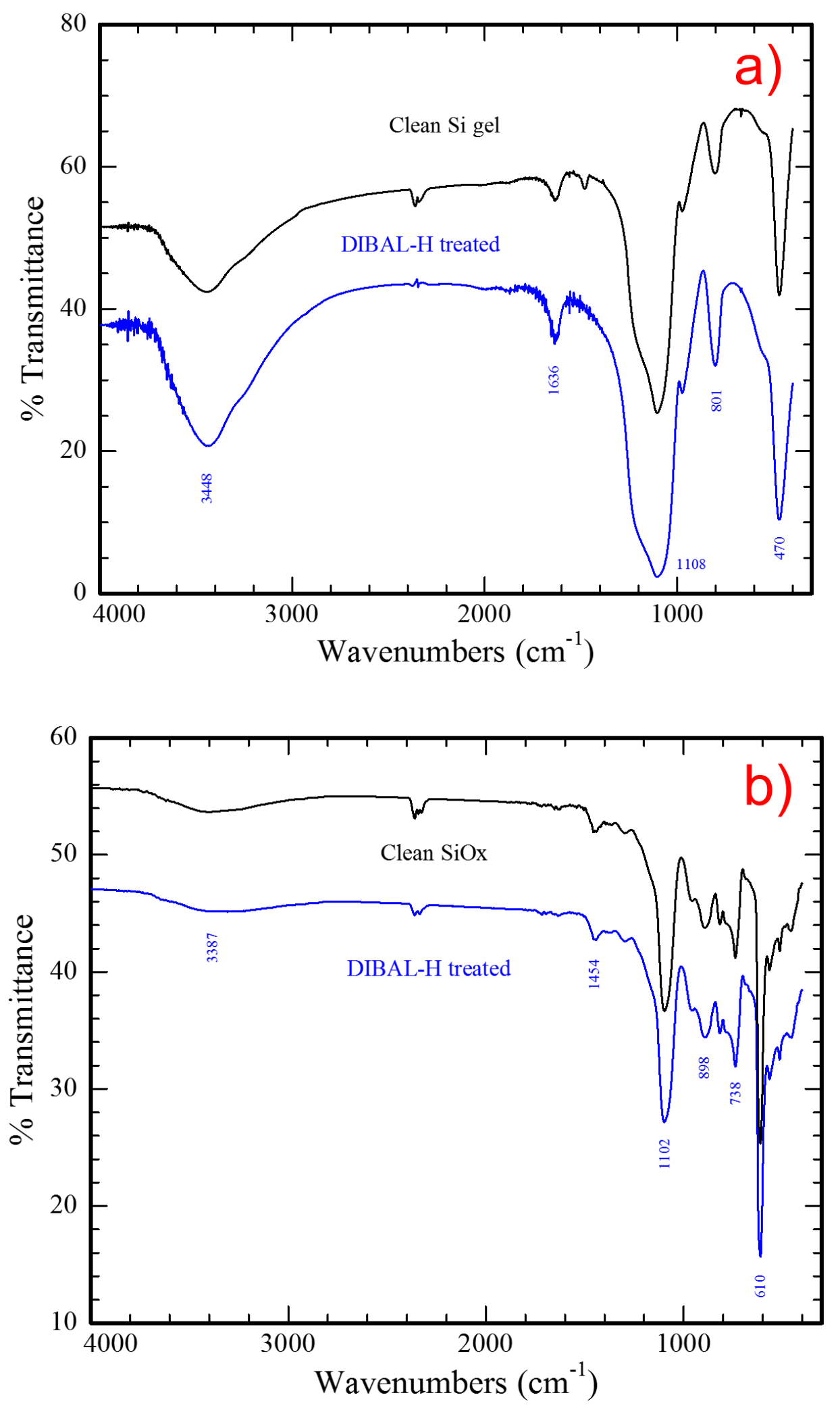


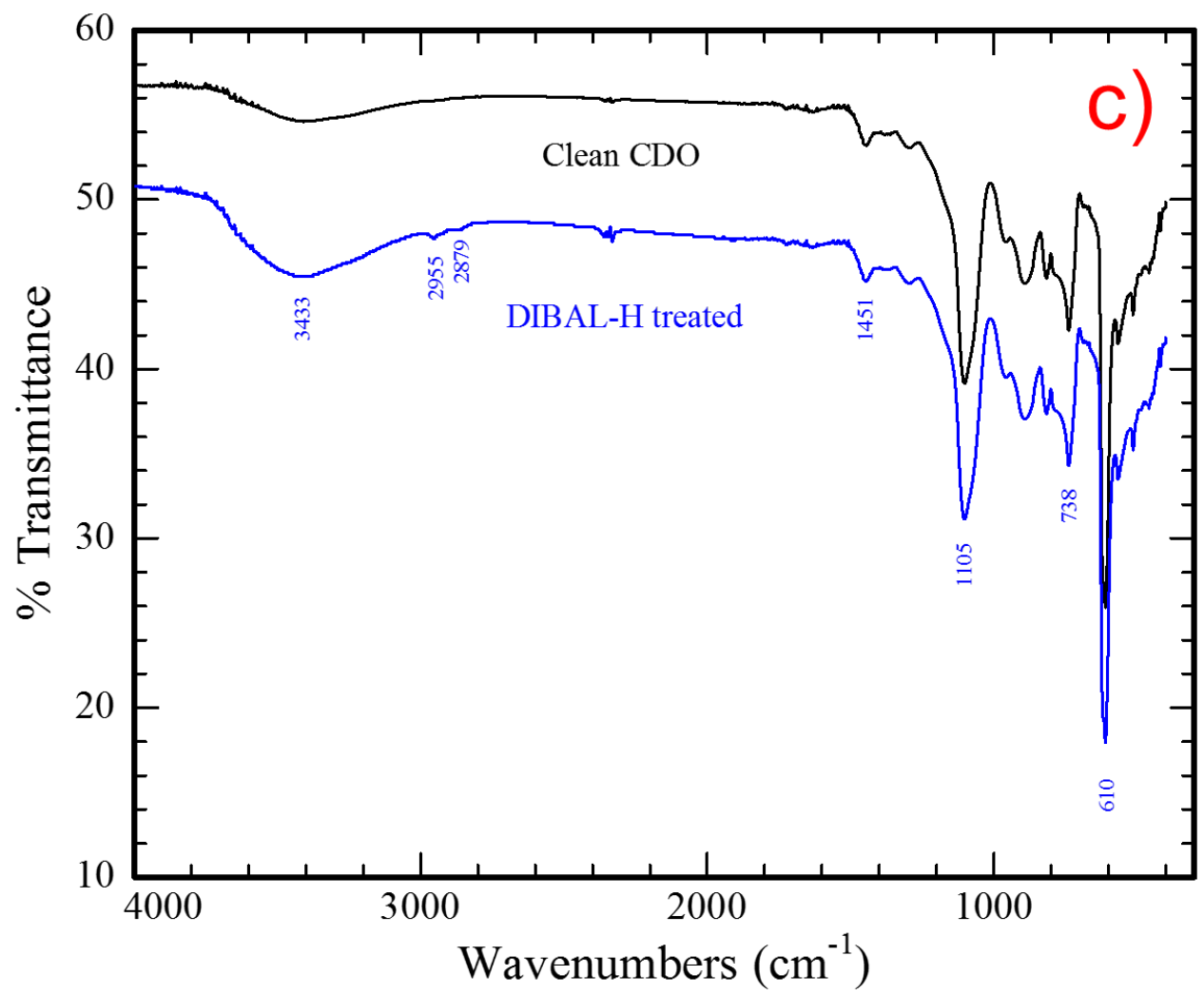

Figure 4.15. Control experiment showing that only the porous structure of CDO traps DIBAL-H from anhydrous toluene solution. A. Silica Gel, B. $\mathrm{SiO}_{\mathrm{x}}$ wafer C. CDO wafer. Only C indicates the presence of C-H stretch vibrations in the $250-2950 \mathrm{~cm}^{-1}$ region.

Like the $\mathrm{SiO}_{\mathrm{x}}$ wafer, $\mathrm{Si}-\mathrm{H}$ stretching peaks were not detected presumably either because of a low surface concentration or rapid degradation in the air ${ }^{109}$ during FT-IR data acquisition. Furthermore, observed weaker IR peak intensities (blue inset in Figure 4.14) for the triflate groups on $\mathrm{CDO}$ and $\mathrm{SiO}_{\mathrm{x}}$ wafer surface compared to silica gel (blue curves in Figures 4.9, 4.12 and 4.14) supported the above interpretation. The FT-IR spectrum after hydrosilylation was similar to the $\mathrm{SiO}_{\mathrm{x}}$ wafer results (cf. red curves in Figures 4.12 and 4.14). A control experiment involving hydrosilylation of underivatized CDO surface revealed a lack of adsorption of octadecene or its photochemical reaction product (Figure 4.16). The observation of DIBAL-H adsorption/trapping (Figure 4.15) but not of 
octadecene on a clean CDO surface (Figure 4.16) implies that a slightly hydrophilic CDO pore is unable to retain nonpolar octadecene, pointing to the amphipathic nature of the pore wall. This weaker pore-octadecene interaction would result in ready removal of octadecene, but not DIBAL-H, during the CDO wafer rinsing/drying stage, before collecting respective IR spectra. In principle, following DIBAL-H reduction of pore surface triflate groups, octadecene can enter CDO pores and derivatize the hydrophobic silicon hydride pore surfaces (red curve Figure 4.14). Alternatively, the steric effects such as nanopore size may preclude entry of the larger octadecane while allowing smaller DIBAL-H molecules. Future studies using smaller size alkenes are planned, however, with the data at hand, we are unable to verify whether the internal pore surface is derivatized or not. 


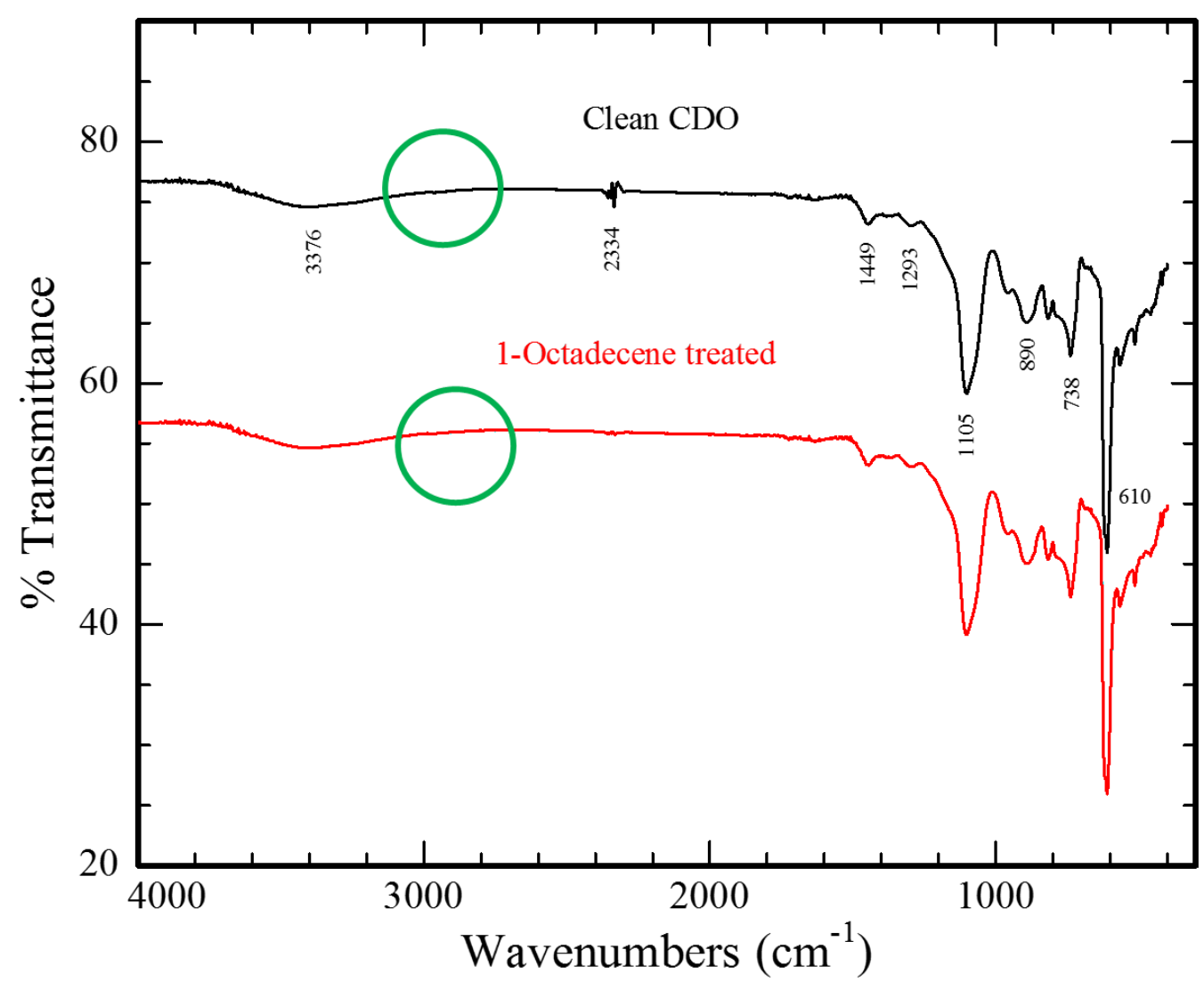

Figure 4.16. FTIR of CDO wafer control experiment. Spectral comparison of clean CDO, i.e., before reaction and CDO after treatment with 1-octadecene in the presence of UV light under identical conditions, were shown. No peaks corresponding to alkyl chains can be observed in 2800 to $3000 \mathrm{~cm}^{-1}$ region.

A complementary technique of X-ray photoelectron spectroscopy (XPS) provided further support for derivatization on CDO wafers. Survey spectra obtained from a CDO surface before (Figure 4.17a) and after (Figure 4.17c) reaction revealed $\mathrm{Si}, \mathrm{C}$, and $\mathrm{O}$ elements. After derivatization, the intensity of the carbon (1s) peak increased, consistent with the derivatization of surface groups with alkyl chains. In deconvoluted high-resolution Si (2p) spectra of clean CDO wafers before the reaction (Figure 4.17b), three strong peaks at $101.6,102.3$ and $103.1 \mathrm{eV}$ corresponding to $\mathrm{Si}-\mathrm{C}$, -Si-O-C- and -O-Si-O-, respectively were observed. The XPS spectrum after the reaction (Figure $4.17 \mathrm{~d}$ ) had the same three 
strong peaks at similar positions but with different intensities. The area of the -O-Si-Opeak decreased from $26 \%$ to $10 \%$ of the $\mathrm{Si}(2 \mathrm{p})$ peak area, and the relative area of $-\mathrm{Si}-\mathrm{C}$ peak increased from $11 \%$ to $22 \%$.

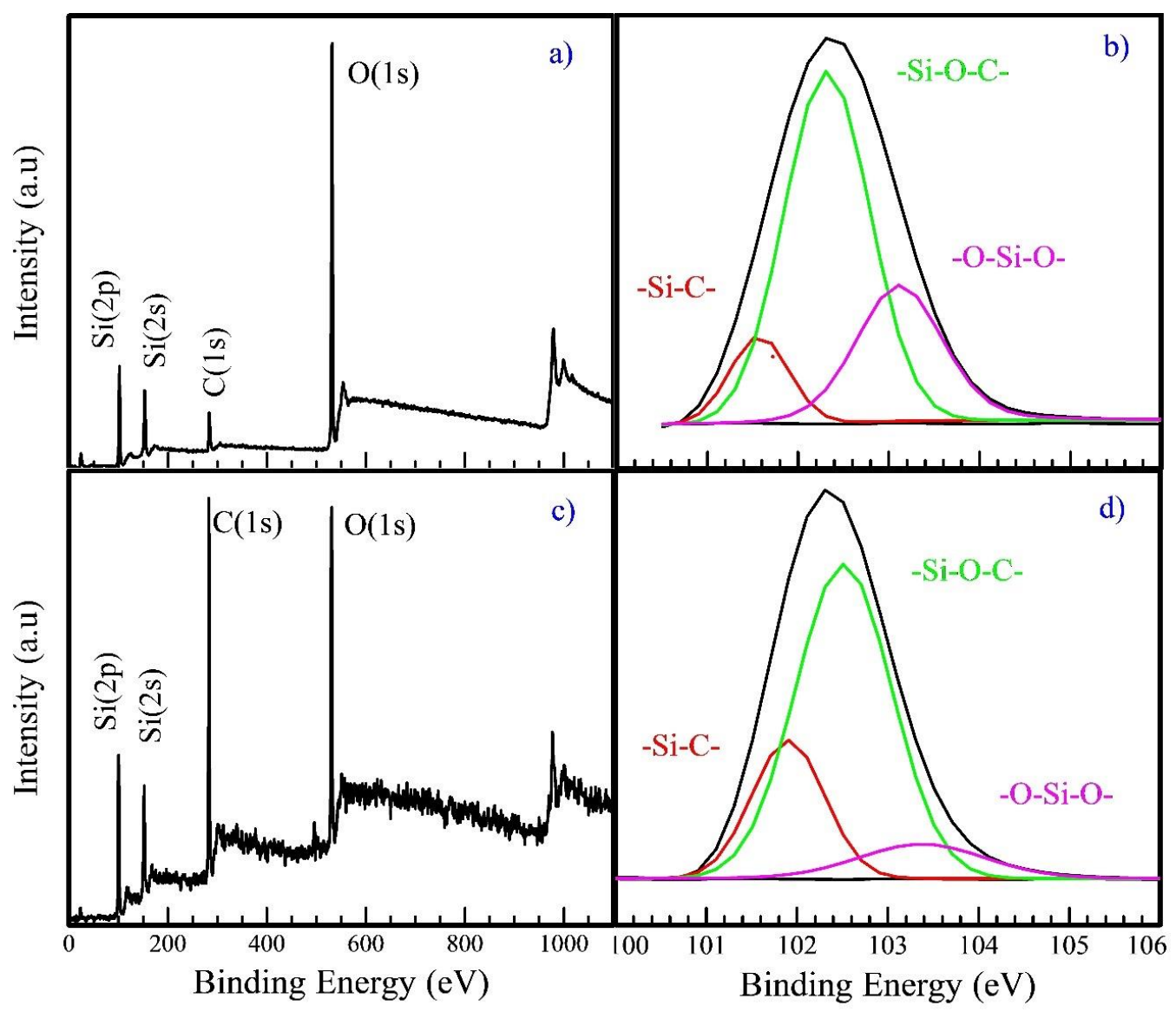

Figure 4.17. XPS spectra of CDO wafer. Survey spectrum, a, and $\mathrm{Si}(2 \mathrm{p})$ high-resolution spectrum, $\mathrm{b}$, collected before the reaction and $\mathrm{c}, \mathrm{d}$ are the corresponding spectra collected after the reaction.

This finding validates the conversion of $-\mathrm{O}-\mathrm{Si}-\mathrm{OH}$ to $-\mathrm{O}-\mathrm{Si}-\mathrm{R}(\mathrm{R}=$ octadecyl). No significant change was observed, however, in the peak area for the -Si-O-C-group, indicating no structural modification in the cage structure of the CDO film. More surface chemical studies are needed to confirm this indication. 
Derivatization of the CDO surface with octadecene should change its hydrophobicity. The contact angle of water droplets deposited on the surface confirmed that shift (Figure 4.18). Clean CDO wafers (Figure 4.18a) had a low contact angle $36 \pm 3^{\circ}$ caused by the presence of the hydrophilic hydroxyl groups $(\mathrm{Si}-\mathrm{OH})$. Derivatization with octadecene increased the contact angle to about $81 \pm 2^{\circ}$ (Figure $4.18 \mathrm{~b}$, Table 4.2 ). The water contact angles on derivatized surfaces were stable for more than a year (See Figure 4.18) as were the corresponding IR spectra (Figure 4.19).
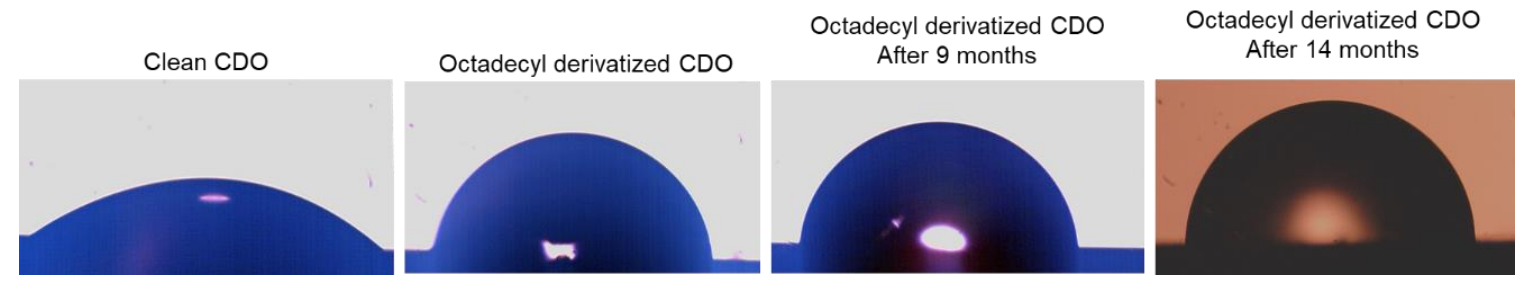

Figure 4.18. Water contact angles measured on un-derivatized (clean CDO) and octadecene derivatized $\mathrm{CDO}$ wafers at various time intervals.

\begin{tabular}{|c|c|}
\hline Substrate & $\begin{array}{c}\text { Contact angle } \\
\text { (deg.) }\end{array}$ \\
\hline Before reaction (Clean CDO) & $36 \pm 3^{\circ}$ \\
After reaction & $81 \pm 2^{\circ}$ \\
9 months later & $82 \pm 3^{\circ}$ \\
14 months later & $82 \pm 2^{\circ}$ \\
\hline
\end{tabular}

Table 4.2. Contact angle results of a water droplet on clean CDO and octadecyl derivatized CDO at various time intervals. 


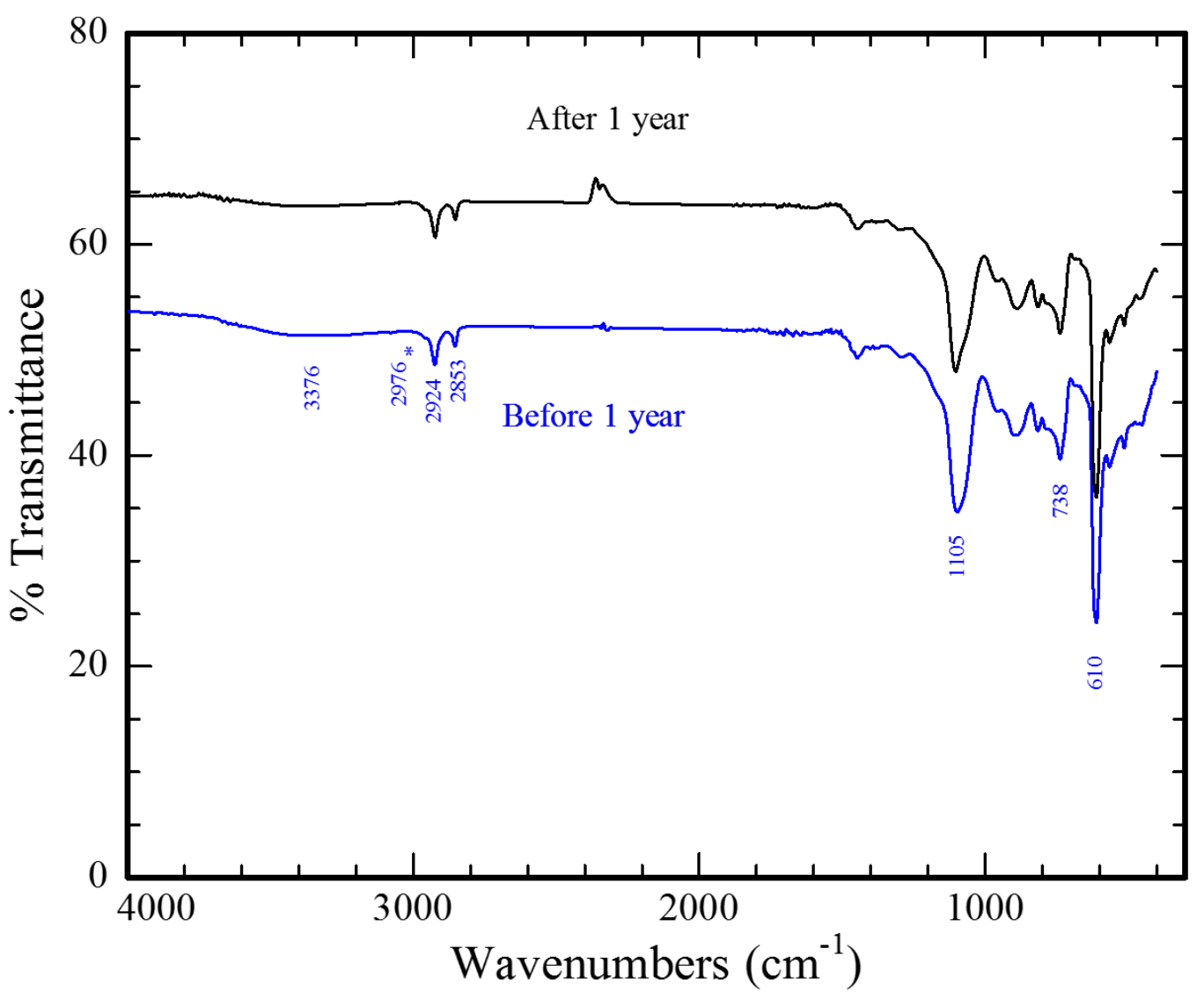

Figure 4.19. FTIR spectra comparison of octadecyl derivatized CDO wafer before and after oneyear duration.

\subsubsection{Comparison of Octadecyl chains derivatized via DIHAL-H chemistry and Octadecyl trichlorosilane (OTS) routes}

Octadecyltrichlorosilane (OTS), a commonly used silanization agent, readily forms a self-assembled monolayer on $\mathrm{SiO}_{\mathrm{x}}$ film transforming it to from hydrophilic to a hydrophobic surface by inserting an alkoxy ether ( $\mathrm{Si}-\mathrm{O}-\mathrm{R})$ group bearing a $\mathrm{C} 18$ alkyl chain. Hence, the results of octadecene-based vs. the OTS-based derivatization on CDO and $\mathrm{SiO}_{\mathrm{x}}$ surfaces were compared. Qualitatively, FT-IR spectra, in the relevant region shown in Figure 4.20, indicated that the thickness of the hydrocarbon layer is higher for octadecene 
derivatization than the OTS SAMs (blue vs. red traces), while the silanol peak intensities were greater in the latter. An increase in $\mathrm{OH}$ peak intensity could come from the free $\mathrm{Si}$ $\mathrm{OH}$ groups on the hydrolyzed OTS molecule as schematically illustrated in Figure 4.20 (inset). These free $\mathrm{O}-\mathrm{H}$ groups provide interfacial hydrophilic sites for water adsorption underneath the alkyl chains of the OTS film. Thus, an insertion of a Si-OR group which has a higher dipole moment than $\mathrm{Si}-\mathrm{R}$ group and new free - $\mathrm{OH}$ groups would be expected to increase the $k$ values of $\mathrm{CDO} /$ Oxide films derivatized using OTS.

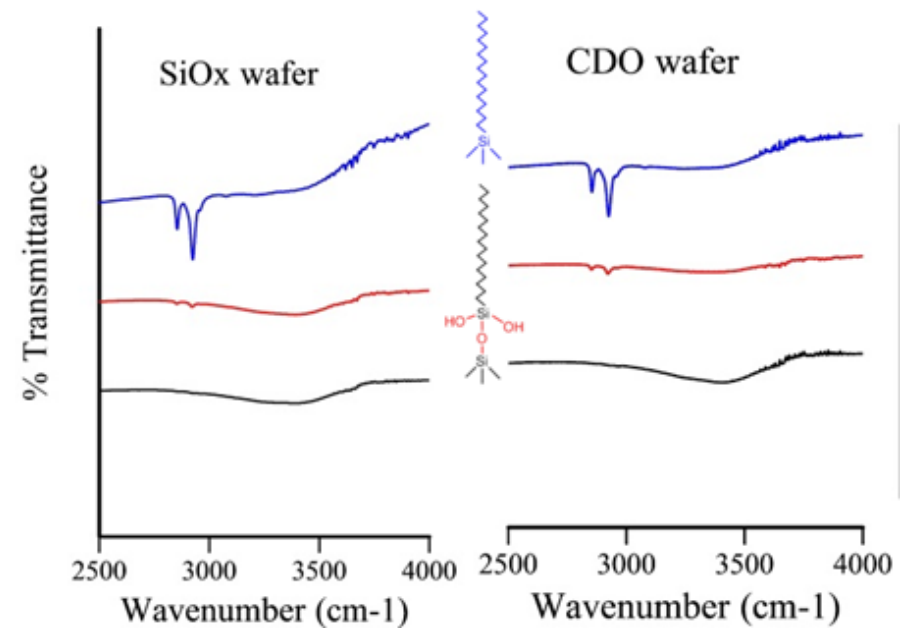

Figure 4.20. Comparison $\mathrm{CDO}$ and $\mathrm{SiO}_{\mathrm{x}}$ wafer derivatized by DIBAL-H assisted chemistry (blue) vs. OTS chemistry (red). Black curves depict IR spectra of the respective wafers before derivatization.

\subsubsection{Characterization of film dielectric constants $(k)$}

Effects of Maxwell-Wagner polarization (MWP) hampered the low frequency (0.1$1 \mathrm{MHz}$ ) electrical measurements of $k$ values due to adsorption of moisture in the nanopores of CDO. Partial dissociation of trapped/adsorbed water molecules imparts to the film a finite electrical conductivity creating electrically leaky (resistive) as opposed to insulating films ${ }^{111}$. Such samples ${ }^{112}$ have a higher dielectric contribution arising from distortion and 
dipolar polarization which raises their effective $k^{113}$. To disentangle MWP and other polarization effects require measurements of the film porosity and frequency dependent dielectric constants as a function of water saturation ${ }^{114}$ (i.e., humidity). Since these data were unavailable, a qualitative consideration of capacitance (proportional to $k(1 \mathrm{MHz})$ ) measurements (Figure 4.21) revealed the following ordering in the magnitude of $k^{\mathrm{OTS}}$ > $k^{\mathrm{CDO}}>k^{\mathrm{DIBAL}-\mathrm{H}}$, consistent with the expected interfacial polarity of these samples (vide supra). One way around this problem is to reduce surface $\mathrm{SiOH}$ group concentration by high-temperature baking in an inert environment.

\section{C-V of three samples}

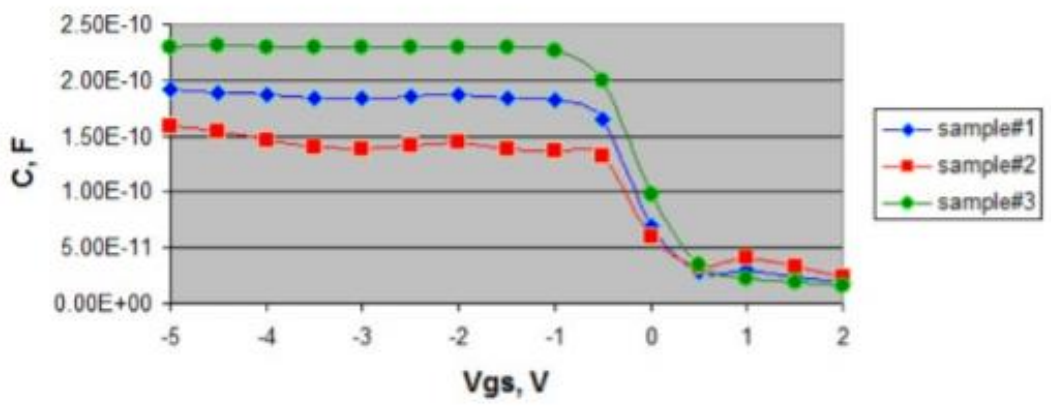

Figure 4.21. Capacitance vs. voltage curves measured using $\mathrm{Hg}$ probe method. $\mathrm{Hg}$ contact area was $3.1 \times 10^{-3} \mathrm{~cm}^{2}$. Samples 1,2 and 3 are underivatized CDO, OTS and Octadecene derivatized $\mathrm{CDO}$ wafers respectively.

Another approach to access $k$ is through measurements of film refractive index; noting that high frequency (optical) $k$ values simply depend on electronic polarization and scale as the square of refractive index ${ }^{115}$. Note, $k$ values at optical frequencies do not contain dielectric contributions from MWP and dipole polarizations ${ }^{113}$ (see below). Analysis of reflectance spectra (Filmetrics) collected from these wafers yielded best-fit values of refractive index and film thickness; tabulated in Table 4.3. Best-fit refractive 
index values for $\mathrm{CDO}$ films were lower than $\mathrm{SiO}_{\mathrm{x}}$ films commensurate with the porous structure of CDO that can trap either water or air, both of which have lower refractive indices than pure $\mathrm{SiO}_{2}$. Furthermore, the effective refractive index values of OTS derivatized films were consistently lower than the octadecene derivatized surface implying perhaps their larger adsorbed moisture content. In either $\mathrm{SiO}_{\mathrm{x}}$ or $\mathrm{CDO}$ films, the refractive index of DIBAL-H-treated films was greater than the OTS-treated films corresponding to its thicker hydrocarbon film of the greater refractive index (1.4439) than refractive indices of either water (1.33) or air (1.0).

To estimate film porosity of CDO crudely, the refractive index of these composite films $\left(\eta_{\exp }\right)$ was decomposed as a sum of volume fraction $(\varphi)$ weighted refractive indices of the constituents ${ }^{116}$ :

$$
\eta_{\exp }=\sum_{i} \eta_{i} \phi
$$

where index $i$ denotes a specific component and $\phi_{i}$ its volume fraction. Assuming that the $\mathrm{CDO}$ matrix is made up of $\mathrm{SiO}_{2}\left(\eta_{\mathrm{SiO} 2}=1.473\right)$ and pores are filled completely with either air $\left(\eta_{\mathrm{SiO} 2}=1\right)$, or water $\left(\eta_{\mathrm{H} 2 \mathrm{O}}=1.333\right)$ gave approximate the effective porosity of 3 or 11 percent for CDO respectively. Within limits of optical measurements of the film thickness using filmetrics, the hydrocarbon layer thickness in the octadecene derivatization scheme was comparable $(\approx 5 \mathrm{~nm})$ for both CDO and $\mathrm{SiO}_{\mathrm{x}}($ Table 4.3). These values were significantly higher than expected for a densely packed upright monolayer of $\mathrm{C} 18$ chain $(2 \mathrm{~nm})$. Ellipsometric measurements, however, consistently gave a higher thickness of hydrocarbon layer in octadecene 
derivatized samples supporting the observed trend of higher IR peak intensities for the C-H stretch vibrations (Figure 4.20). These higher IR intensities are consistent with partial cross-linking/polymerization of octadecene during the hydrosilylation $\operatorname{step}^{117}$.

\begin{tabular}{|c|c|c|c|c|c|c|c|}
\hline \multirow[t]{2}{*}{ Sample } & \multirow[t]{2}{*}{ 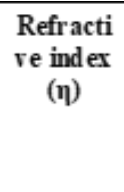 } & \multirow[t]{2}{*}{$\begin{array}{c}k^{\text {opt }}= \\
\eta^{2}\end{array}$} & \multirow{2}{*}{$\begin{array}{c}\text { Overlayer } \\
\text { Thickness } \\
\text { (nm) } \\
\text { Filmetrics }\end{array}$} & \multirow{2}{*}{\begin{tabular}{|c|}
$\begin{array}{c}\text { Orerlayer } \\
\text { Thickness } \\
\text { (nm) }\end{array}$ \\
Ellipsometry
\end{tabular}} & \multicolumn{2}{|c|}{$\begin{array}{l}\text { Hydroc arbon } \\
\text { hyer thic kness } \\
\text { (nm) }\end{array}$} & \multirow[t]{2}{*}{$\begin{array}{c}\mathrm{CA} \\
\text { (deg.) }\end{array}$} \\
\hline & & & & & $\begin{array}{c}\text { Film- } \\
\text { metrics }\end{array}$ & $\begin{array}{l}\text { Ellipso } \\
\text { metry }\end{array}$ & \\
\hline CDO clean & 1.457 & 2.12 & $101.2 \pm 0.6$ & $102.9 \pm 0.6$ & 0 & 0 & $36 \pm 3^{2}$ \\
\hline $\begin{array}{l}\text { 1-Octa decene } \\
\text { deriv. CDO } \\
\text { through DBAL-H }\end{array}$ & 1.447 & 2.09 & $103.1 \pm 2.4$ & $123 \pm 4$ & $2 \pm 3$ & $10 \pm 5$ & $87 \pm 3^{\circ}$ \\
\hline OTS deriv. $\mathrm{CDO}$ & 1.412 & 1.99 & $106.4 \pm 0.3$ & $107.2 \pm 0.9$ & $5 \pm 1$ & $4 \pm 2$ & $91 \pm 4^{\circ}$ \\
\hline $\mathrm{SiO}_{\mathrm{x}}$ clean & 1.473 & 2.17 & $107 \pm 2$ & $115.1 \pm 0.3$ & 0 & 0 & $11 \pm 2^{\circ}$ \\
\hline $\begin{array}{l}\text { 1-Octadecene } \\
\text { deriv. SiO through } \\
\text { DIBAL-H }\end{array}$ & 1.448 & 2.09 & $111.2 \pm 0.9$ & $117.0 \pm 2.4$ & $4 \pm 3$ & $2 \pm 3$ & $101 \pm 4^{2}$ \\
\hline OTS deriv. $\mathrm{SiO}_{x}$ & 1.435 & 2.06 & $118.9 \pm 0.6$ & $117.0 \pm 0.9$ & $2 \pm 3$ & $2 \pm 1$ & $105 \pm 3^{\circ}$ \\
\hline
\end{tabular}

Table 4.3. Contact angle, film thicknesses of $\mathrm{CDO}$ and $\mathrm{SiO}_{\mathrm{x}}$ wafers.

The contact angle measurements revealed similar trends. The contact angles for both derivatization schemes depended on the nature film, i.e., $\mathrm{CDO}$ vs. $\mathrm{SiO}_{\mathrm{x}}$ and not on the chemical mode of derivatization, i.e., silanization vs. hydrosilylation. The CDO surface which had higher contact angle before derivatization $\left(36^{\circ}(\mathrm{CDO})\right.$ vs. $\left.11^{\circ}\left(\mathrm{SiO}_{\mathrm{x}}\right)\right)$ but exhibited lower contact angle after derivatization $\left(87^{\circ}\right.$ vs. $91^{\circ}$ for CDO derivatized with octadecene and OTS respectively). Applying the Cassie equation for a measured contact angle $\left(\theta_{m}\right)$ on a surface made up of two types of 
regions (labeled with superscripts 1 and 2) with differing contact angles, $\theta_{1}$ and $\theta_{2}$, gives $^{118}$ :

$$
\cos \theta_{m}=f\left(\cos \theta_{1}-\cos \theta_{2}\right)+\cos \theta_{2}
$$

where $f$ represents a fraction of the surface of type 1 . Crudely, identifying region 1 as $\mathrm{SiO}_{\mathrm{x}}\left(\theta_{1}=11^{\circ}\right)$ and region 2 as pores (i.e., air with $\left.\theta_{2}=180^{\circ}\right)$ on the $\mathrm{CDO}$ surface, the estimated upper limit for $f=0.91$ implying a 9 percent surface porosity. Should the lower $\mathrm{OH}$ density on the CDO surface be accounted for by setting $\left(\theta_{1}>11^{\circ}\right)$ the measured porosity would decrease. For example, if $\theta_{1}=30^{\circ}$ for the nonporous CDO surface, then the calculated pore fraction would be 3 percent, similar to the estimates from the refractive index measurements (Vide Supra). Since we did not have a nonporous CDO sample, we did not have a value for $\theta_{l}$.

For $\mathrm{SiO}_{\mathrm{x}}$ surface the observed contact angles after derivation were $101^{\circ}$ (octadecene) vs. $104^{\circ}$ (OTS) implying a more densely packed monolayer as was noted elsewhere ${ }^{119}$. Again, applying the Cassie equation, but now ascribing region 1 to the densely packed hydrocarbon monolayer $\left(\theta_{1}=107^{\circ}\right)$ and region 2 to underivatized $\operatorname{SiO}_{\mathrm{x}}\left(\theta_{2}=11^{\circ}\right.$, from Table 1$)$, estimated $f$ values were 0.97 for OTS and 0.92 for Octadecene derivatized $\mathrm{SiO}_{\mathrm{x}}$ surfaces. By contrast, the CDO surface had a thicker but more loosely packed hydrocarbon layer due to the lower density of surface $\mathrm{OH}$ groups, inferred from its higher initial contact angle. The corresponding $f$ values on the CDO surfaces were, as expected, smaller; 0.75 (OTS) and 0.69 (Octadecene) respectively. The results also illustrated that the hydrocarbon layer thickness did not affect the contact angle, but the areal fraction of derivatized (i.e., $f$ ) surface did. 
The reaction of hydrofluoric acid (HF) can also enhance the hydrophobicity of the silicon surface by creating a hydrogen-terminated surface that may be hydrosilylated, but it would produce undesirable etching of the CDO film. Recently, our group has used sacrificial silanes to prevent the etching of $\mathrm{SiO}_{\mathrm{x}}$ by $\mathrm{HF}$. The chemistry, however, is carried out in an aqueous medium of low $\mathrm{pH}$, which would likely reoxidize $\mathrm{Si}-\mathrm{H}$ groups ${ }^{2}$ and hence is undesirable for this protecting the inner layer dielectrics from moisture.

\subsection{Thiol functionalization of CDO surface to enhance NWs interaction to substrate}

Thiol functional groups(-SH) shows strong interaction towards noble and coinage metals $^{120}$. Thiol interaction and formation of self-assembled monolayers (SAM) of alkane thiols on copper has been extensively studied ${ }^{120-123}$. Thiol functionalization of CDO surface was explored to enhance the chemical interaction of as-deposited $\mathrm{Cu} / \mathrm{Ni} \mathrm{NWs}$ to $\mathrm{CDO}$ substrate.

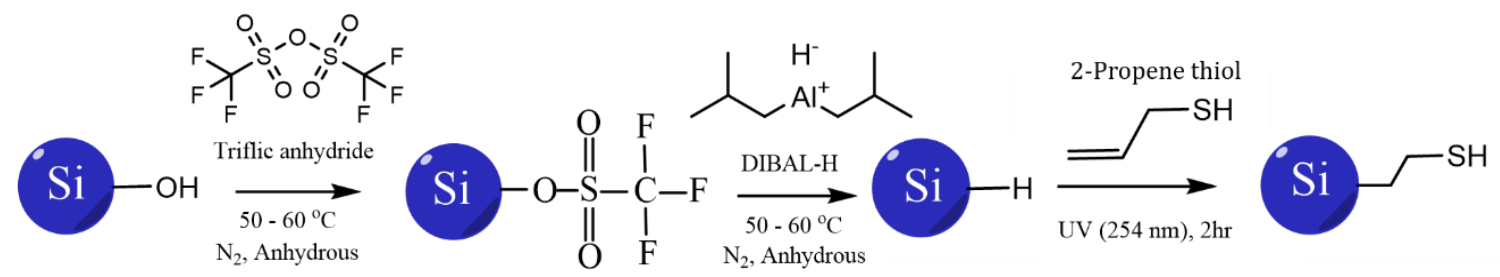

Scheme 4.4. Reaction scheme describing thiol functionalization of CDO surface.

Thiol functionalization of CDO was carried out with the DIBAL-H approach, See Scheme 4.4. The reaction was carried out under identical conditions, except 1-Octadecene is replaced with a 2-propene thiol in the photochemical reaction. In the FTIR characterization, 2888 and $2941 \mathrm{~cm}^{-1}$ peaks are attributed to propyl chain (3 carbon chain) 
and $2564 \mathrm{~cm}^{-1}$ is a characteristic peak corresponding to thiol (-S-H) stretch suggests the thiol termination, See Figure 4.22.

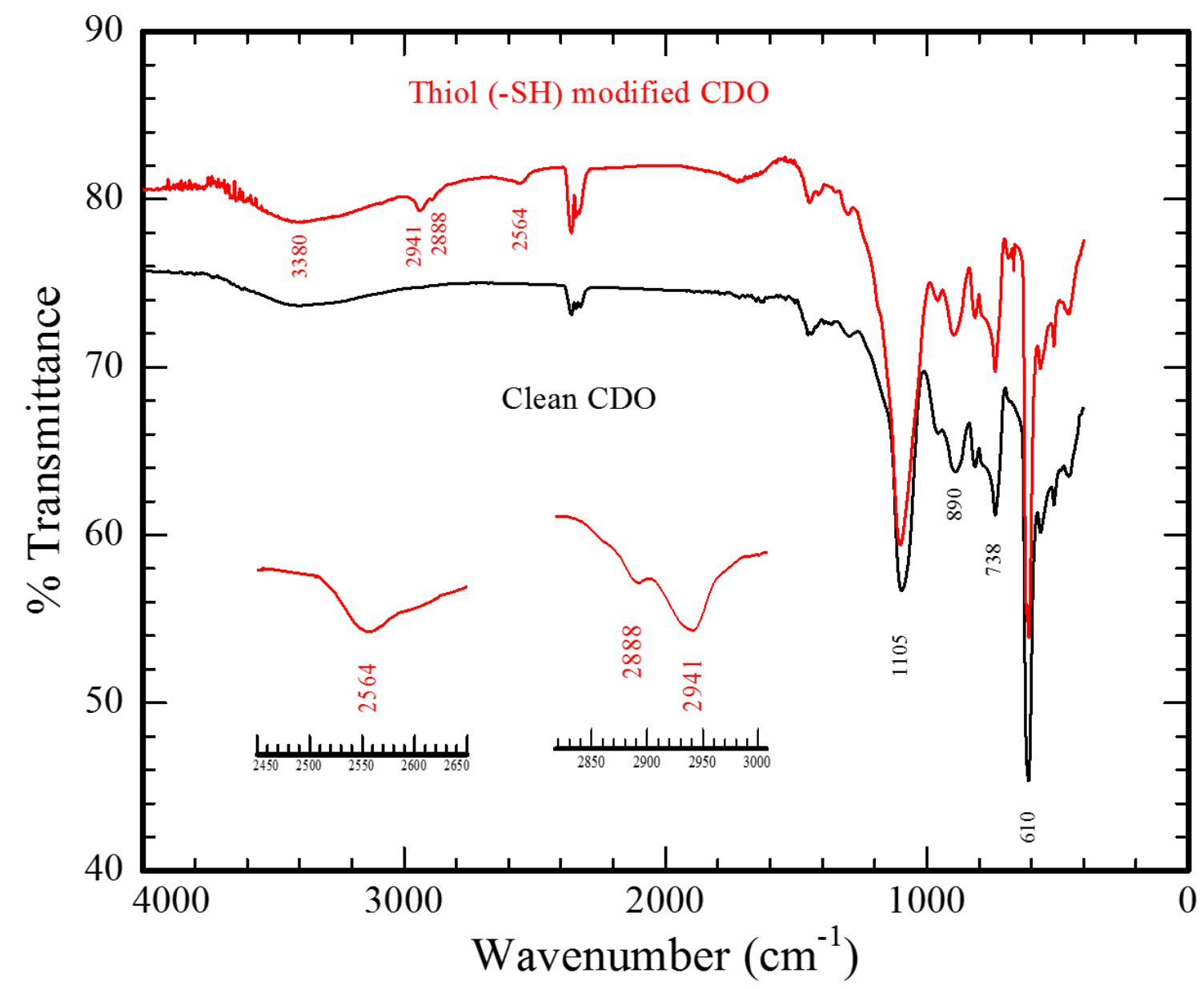

Figure 4.22. FTIR characterization of as received CDO (black curve) and thiol (-SH) functionalized CDO (red curve).

Adhesion strength of $\mathrm{Cu} / \mathrm{Ni} \mathrm{NWs}$ to thiol (-SH) functionalized CDO surface: The main purpose of thiol functionalization is to strongly hold the $\mathrm{Cu} / \mathrm{Ni} \mathrm{NWs}$ after deposition onto CDO surface. The adhesion strength of as received CDO (non-functionalized) vs. thiol functionalized CDO was qualitatively assessed by ultra-sonic treatments, see set up in Figure 4.23a. The procedure involves, drop casting of equal amounts of $\mathrm{Cu} / \mathrm{Ni} \mathrm{NWs}$ 
solution (250 $\mu \mathrm{L}, 5 \mathrm{mg} / \mathrm{mL}$ NWs concentration) on as received CDO and thiolfunctionalized CDO ( $2 \times 2 \mathrm{~cm}$ coupons). After drying, wafers substrates were immersed in ethanol solvent in a beaker and placed in ultrasonicator (160 watts); Figure 4.23a. In case of non-functionalized CDO, traces of NWs were washed off within 4 seconds of ultrasonic treatment, see Figure 4.23b. Whereas for thiol functionalized CDO, holds the NWs strongly till 30 seconds of sonication treatment, see Figure 4.23c. And, after 150 seconds of ultrasonic treatments traces of NWs were washed off, this suggests the enhanced interaction of NWs to thiol functionalized CDO surface.

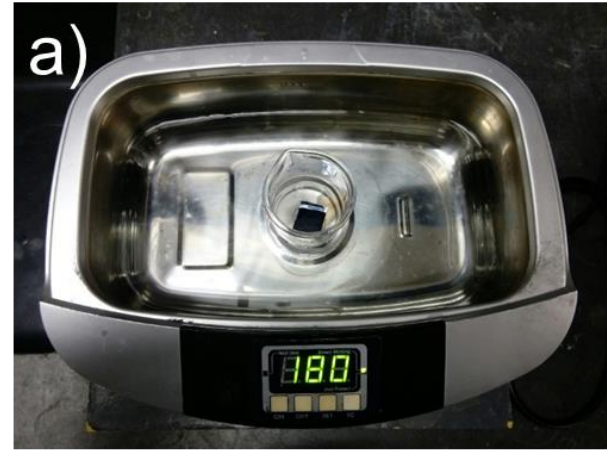

Thiol-modified CDO

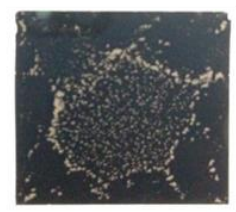

0 sec

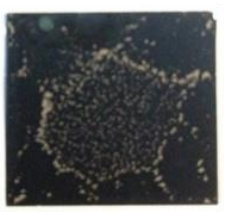

$30 \mathrm{sec}$
Non-modified CDO

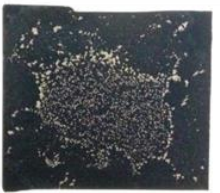

$0 \mathrm{Sec}$

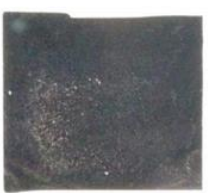

$2 \mathrm{sec}$ b)

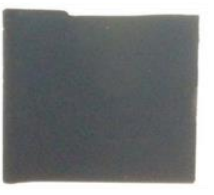

$4 \mathrm{sec}$

\section{C)}

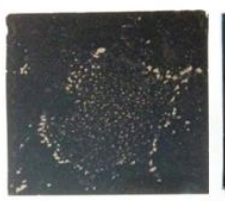

$60 \mathrm{sec}$

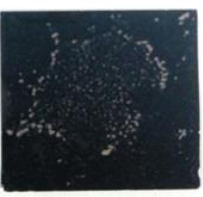

$90 \mathrm{sec}$

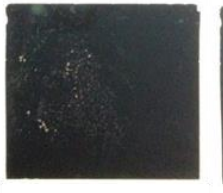

$120 \mathrm{sec}$

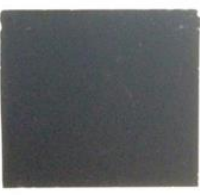

$150 \mathrm{sec}$

Figure 4.23. Digital photograph showing ultrasonication setup (a), for qualitatively testing the adhesion strength of $\mathrm{Cu} / \mathrm{Ni} \mathrm{NWs}$ to as received $\mathrm{CDO}$ (b), and thiol-functionalized CDO surface (c). 


\subsection{Summary of octadecyl and thiol functionalization}

The current plasma treatments to prevent uptake of atmospheric moisture by low dielectric CDO cause thinning of the films. The classical HMDS derivatization to make surfaces hydrophobic does not provide sufficient protection. The studies presented here show a selective derivatization of surface hydrophilic $\mathrm{Si}-\mathrm{OH}$ groups to form a hydrophobic alkyl-modified silicon $\left(\mathrm{Si}-\mathrm{R}, \mathrm{R}=-\mathrm{C}_{18} \mathrm{H}_{37}\right)$ without disturbing the rest of the cage structure of the CDO film. This selective hydrophobic modification should help maintain the ultralow $\mathrm{k}$ of the insulating film. The method does require an additional processing step to remove pore-trapped DIBAL-H reagent that can be removed readily with mild acetic acid. The versatile method allows derivatization of $\mathrm{Si}-\mathrm{OH}$ functional groups on the surface of silica gel or CDO wafers with a variety of other organic molecules useful in designing other

biochips, sensors ${ }^{124-126}$. Preliminary studies indicate that the degree of hydrophobicity created on surface compares favorably with the classic silanization approach, bypassing interfacial insertion of the polar Si-O-R group. It provides acceptable thermal processing stability for the interconnect processing. The same DIBAL-H reduction approach was used for the thiol (-SH) functionalization. And, the qualitative analysis by ultra-sonic treatments suggests the strong adhesion of $\mathrm{Cu} / \mathrm{Ni} \mathrm{NW}$ s to the thiol-modified CDO surface. 


\section{CHAPTER FIVE}

\section{NANOWIRES SELF-ASSEMBLY AND ELECTRICAL CHARACTERIZATION}

Over the last two decades, substantial efforts have been devoted to the assembly of 1D nanostructures into nanoscale circuits and interconnects ${ }^{38,41-43,48,127}$. In this chapter, the self-assembly of $\mathrm{Cu} / \mathrm{Ni} \mathrm{NWs}$ into an interconnect structure on a $\mathrm{CDO}$ wafer using the combination of photolithography and magnetic field assisted NWs alignment techniques is presented. CDO is an important nanoporous low- $k$ material being used as an inner layer dielectric (ILD) in advanced ultra-large scale integrated circuits (ULSI) ${ }^{60,} 128$. The NWs self-assembly protocol discussed here on CDO can also be applied on to the traditional silicon dioxide $\left(\mathrm{SiO}_{\mathrm{x}}\right)$ substrate.

The Strong interaction between the substrate and deposited NWs is essential for the successful assembly of $\mathrm{NWs}^{129}$. Here the chemical interaction of thiol (-SH) functional groups to copper and nickel was exploited to strongly adhere the NWs after deposition onto the $\mathrm{CDO}$ substrate. $\mathrm{CDO}$ surface was functionalized with dangling thiol (-SH) functional groups using the protocol described in chapter 4 , section $4.6^{129}, 130$. Thiol functionalization and its characterization were discussed in detail in chapter $4^{130}$.

\subsection{Self-assembly of $\mathrm{Cu} / \mathrm{Ni} \mathrm{NWs}$ into interconnects}

Schematic diagram shown in Figure 5.1 describes the process flow for the fabrication of $\mathrm{Cu} / \mathrm{Ni} \mathrm{NWs}$ based interconnects. The protocol involves first transferring the desired

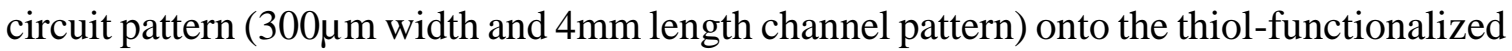
CDO substrate using a photolithography process, see Section 2.2.5 for more details about the photolithography process. The above-patterned substrate was placed in between the 
electromagnet poles like the schematic shown in Figures 3.10 and 5.1. Cu/Ni NWs aqueous solution ( $100 \mu \mathrm{L}$ of $0.5 \mathrm{mg} / \mathrm{mL}$ solution) was placed on to the substrate and allowed to dry in the presence of magnetic field $(2500 \mathrm{G})$. In the presence of magnetic field, NWs oriented in the applied magnetic field direction and begin to form NW chains in the solution; see Figure 3.12. After allowing the solvent to dry under ambient conditions, well aligned NWs deposited on to the surface of photoresist as well as in the thiol-modified trenches on CDO. The NWs which were deposited in the trenches are strongly held to that surface through the covalent bond to the thiol functional groups on CDO surface. The NWs which were deposited on photoresist were loosely bound. These loosely bound NWs and photoresist were removed by washing with acetone solvent (the so-called lift-off process). Acetone solvent dissolved the photoresist and simultaneously washed off the loosely bound NWs from the CDO surface. Finally, the selectively deposited and well-aligned $\mathrm{Cu} / \mathrm{Ni}$ NWs in the circuit pattern remained on the substrate like the optical micrographs shown in Figure 5.2. 


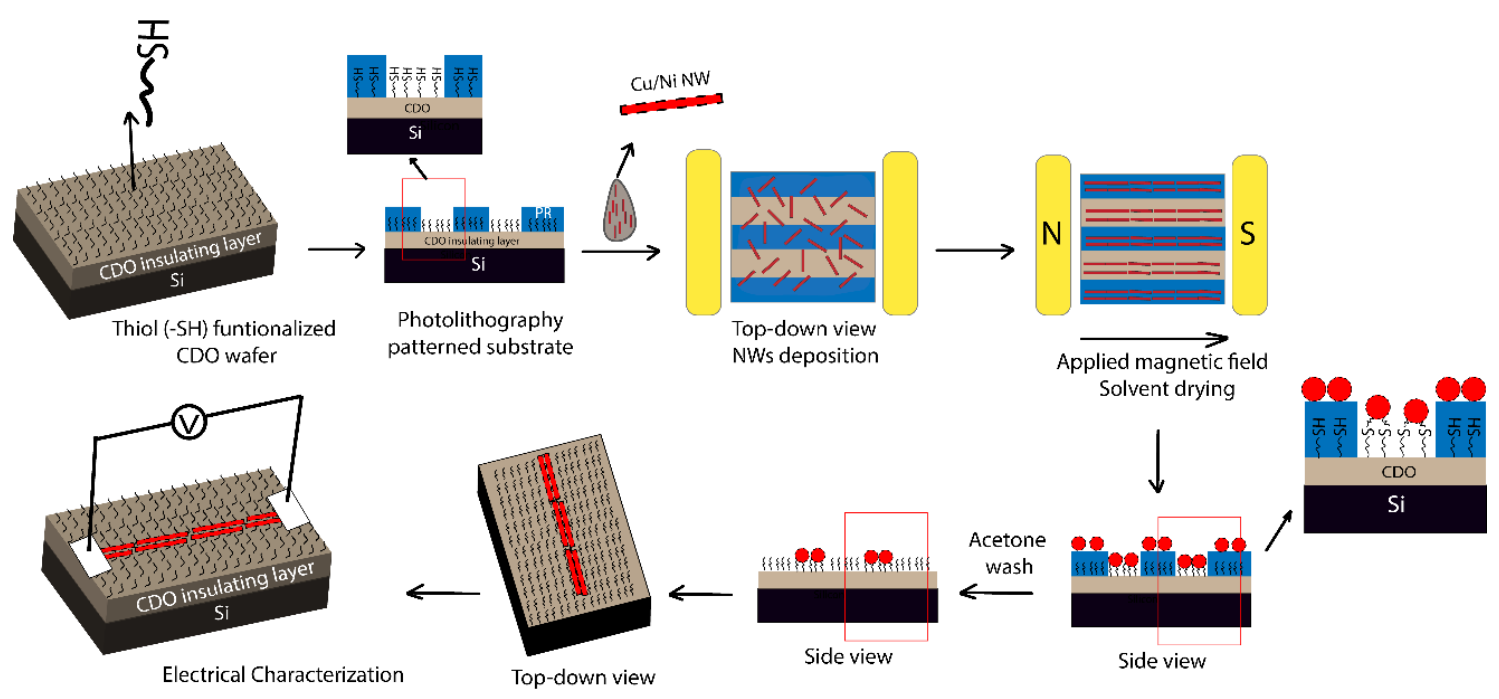

Figure 5.1. Schematic diagram showing a process flow of $\mathrm{Cu} / \mathrm{Ni} \mathrm{NWs}$ self-assembly into interconnect channels.

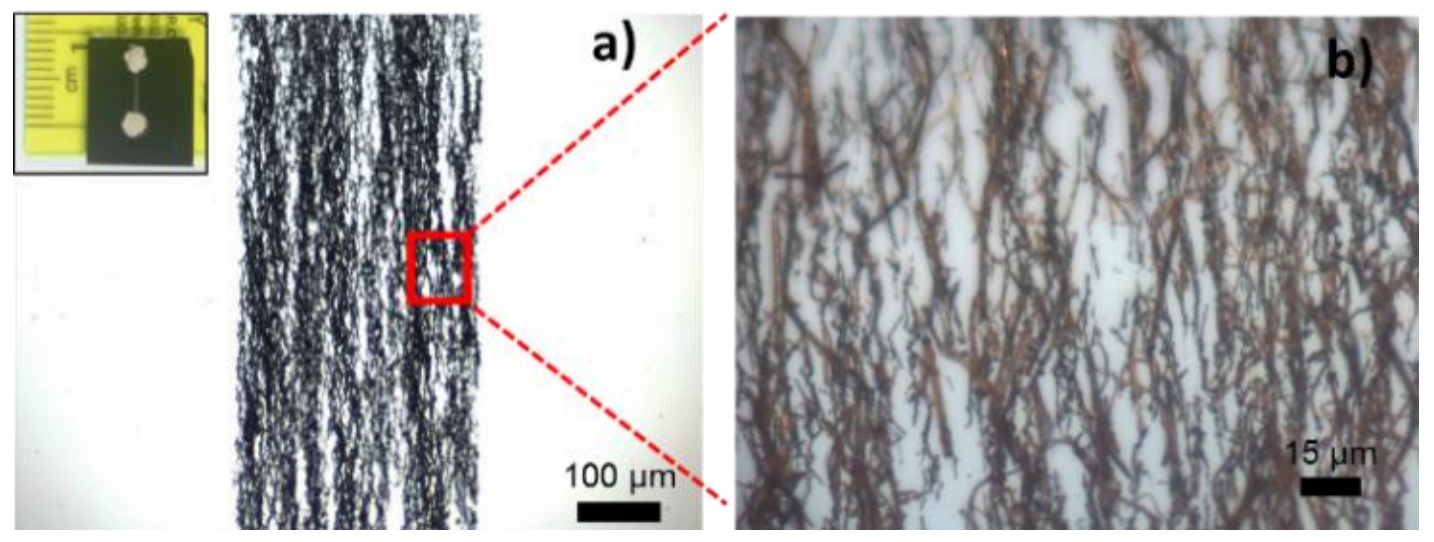

Figure 5.2. Optical micrographs of unidirectionally aligned $\mathrm{Cu} / \mathrm{Ni} \mathrm{NWs}$ interconnect channel fabricated by depositing $0.5 \mathrm{mg} / \mathrm{mL}$ of NWs solution on pattern substrate. Inset image in a) shows digital photograph of $4 \mathrm{~mm}$ length and $300 \mu \mathrm{m}$ width NWs channel with silver ink electrodes on both ends of the channel.

\subsection{Electrical characterization of $\mathrm{Cu} / \mathrm{Ni} \mathrm{NWs}$ based interconnect channels}

\subsubsection{Effect of nanowire type (pure $\mathrm{Cu}$ NWs vs. $\mathrm{Cu} / \mathrm{Ni} \mathrm{NWs}$ ) and orientation (random vs. aligned) on channel resistance.}

Electrical characterization studies of as-fabricated NWs channels were carried out by measuring the electrical resistance using a Keithley-617 programmable digital 
multimeter. Contact pads on both ends of the interconnect channel were fabricated by depositing a small amount $(20 \mu \mathrm{L})$ of silver ink, see inset image in Figure 5.2a. The contact resistance of the dry silver ink was observed as $<1 \Omega$, negligible in comparison to the resistance of interconnect channels. For the comparison, along with the well-aligned $\mathrm{Cu} / \mathrm{Ni}$ NWs deposited in the channel, several samples were fabricated with similar dimensions of interconnect channels wherein randomly arranged $\mathrm{Cu} / \mathrm{Ni}$ and pure $\mathrm{Cu} \mathrm{NWs}$ were deposited. All the above-fabricated channels used $0.5 \mathrm{mg} / \mathrm{mL}$ (dry mass of NWs) concentration of NWs in aqueous suspensions. The common channel lengths and widths were $4 \mathrm{~mm}$ and $300 \mu \mathrm{m}$ respectively. Schematic diagrams, digital photographs and optical micrographs of the as mentioned NWs interconnect channels appear in Figures 5.4, 5.4, 5.5 \& 5.6.

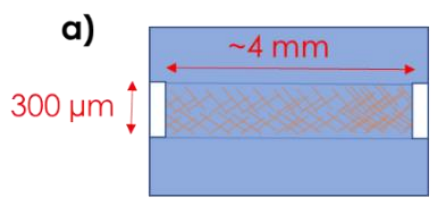

Pure Cu NWs

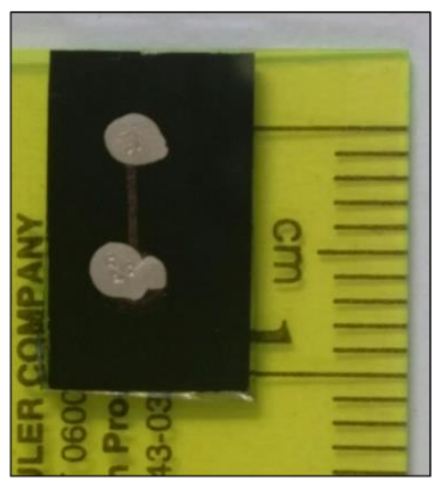

b)

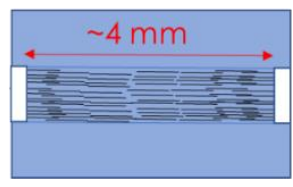

$\mathrm{Cu} / \mathrm{Ni}$ NWs

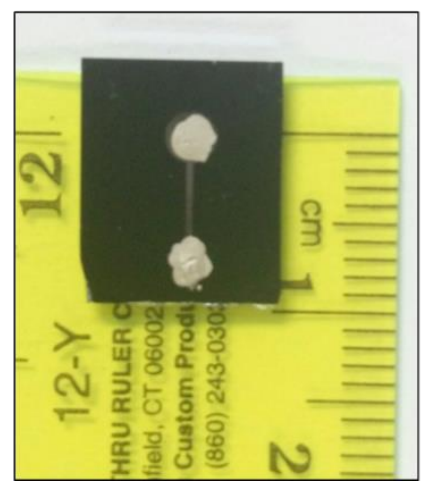

c)

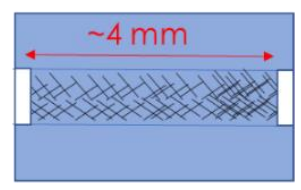

Cu/Ni NWs

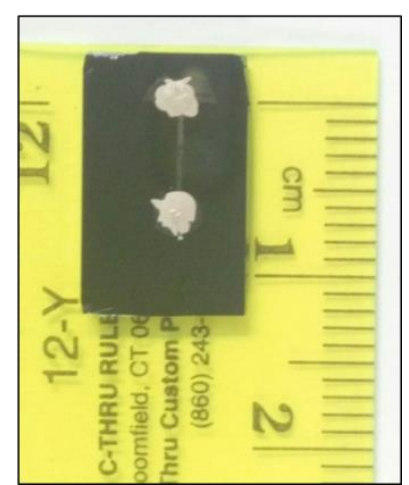

Figure 5.3. Schematic diagrams and digital photographs showing interconnect channels fabricated by using different types and arrangement styles of NWs. The random arrangement of $\mathrm{Cu}$ NWs channel in a), unidirectionally aligned $\mathrm{Cu} / \mathrm{Ni} \mathrm{NWs}$ channel in b), and randomly arranged $\mathrm{Cu} / \mathrm{Ni}$ NWs channel is shown in c). 

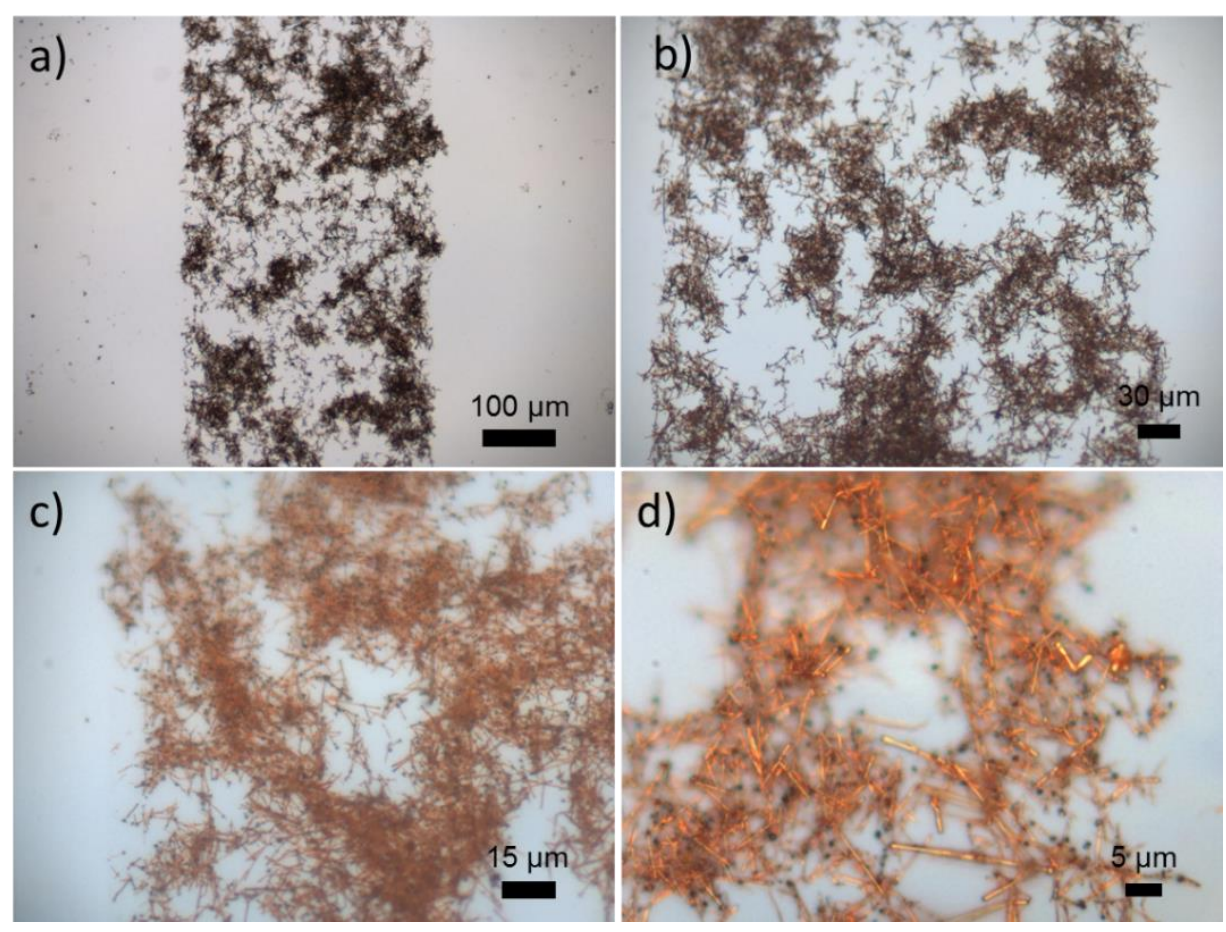

Figure 5.4. Optical micrographs of $\mathrm{Cu}$ NWs based interconnect channel at various magnification scales showing the random arrangement of Cu NWs.

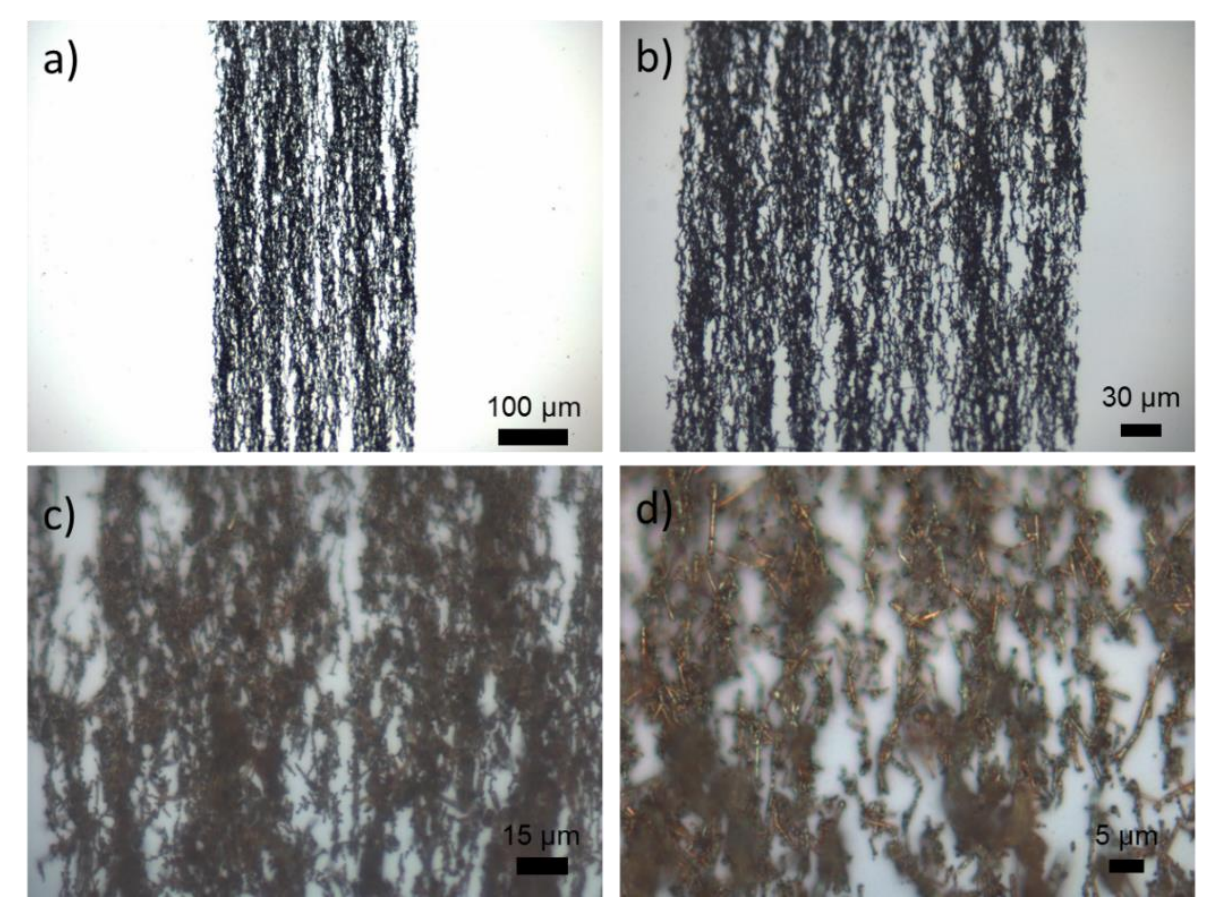

Figure 5.5. Optical micrographs of $\mathrm{Cu} / \mathrm{Ni} \mathrm{NWs}$ based interconnect channel at various magnification scales showing directionally well-arranged NWs. 

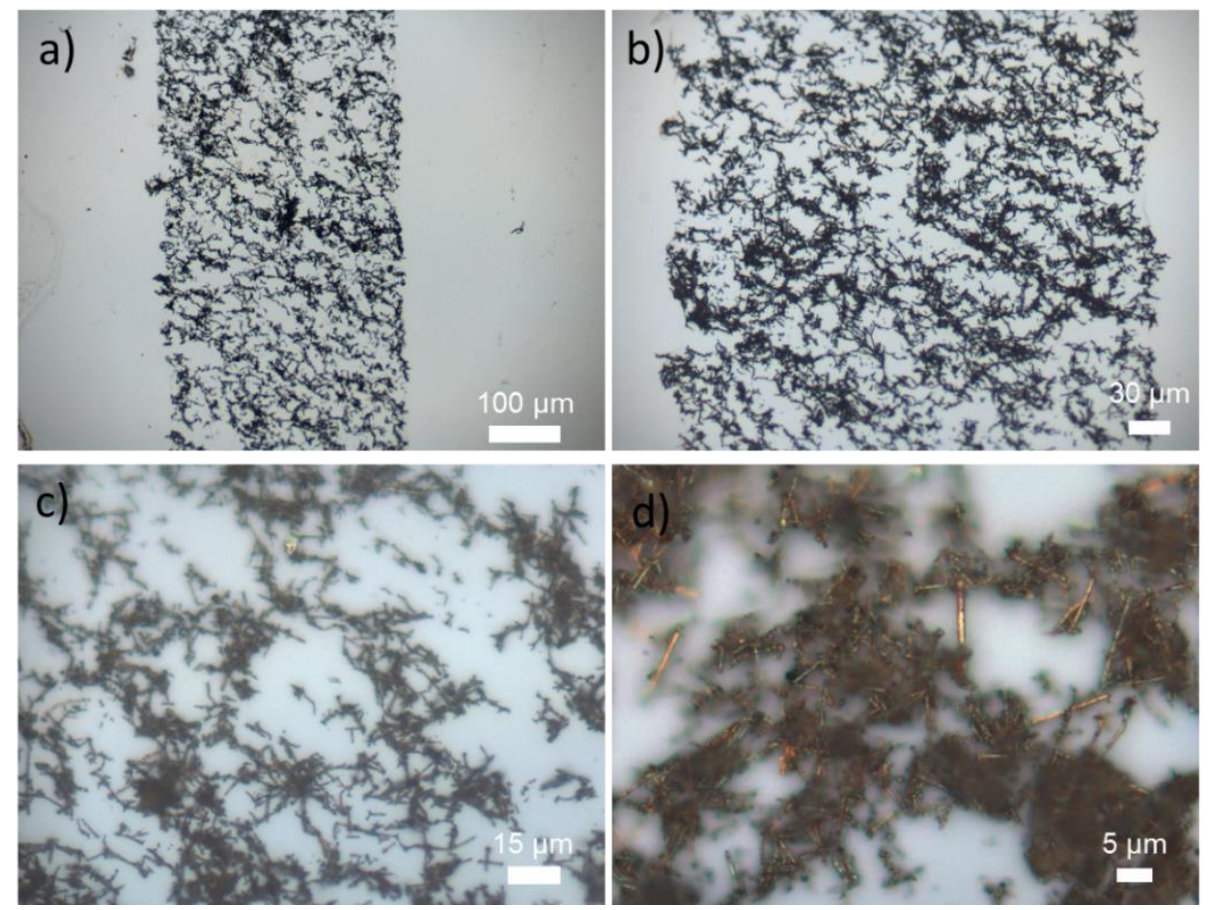

Figure 5.6. Optical micrographs of $\mathrm{Cu} / \mathrm{Ni} \mathrm{NWs}$ based interconnect channel at various magnification scales showing randomly arranged NWs.

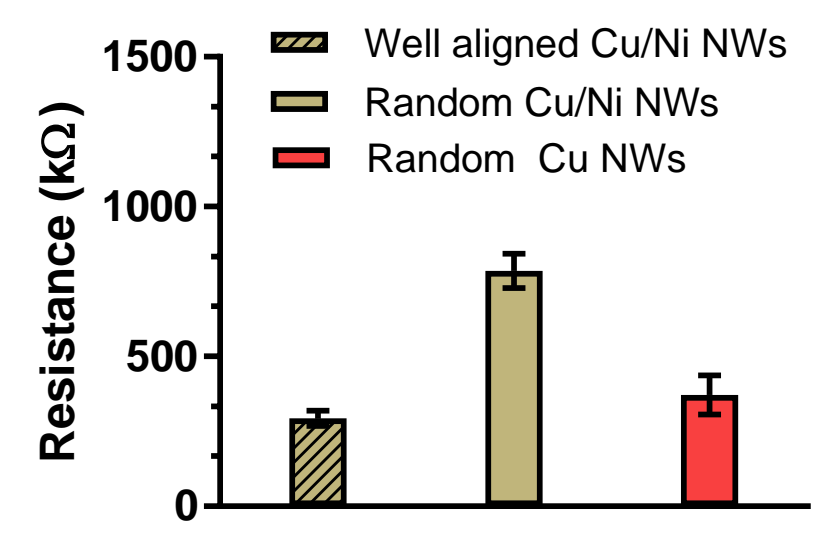

Figure 5.7. Plot showing resistance values of well-aligned and randomly arranged $\mathrm{Cu} / \mathrm{Ni} \mathrm{NWs}$, and randomly arranged pure $\mathrm{Cu}$ NWs based interconnect channels.

The average resistance values of interconnect channels which were made of wellaligned $\mathrm{Cu} / \mathrm{Ni} \mathrm{NWs}$, and randomly arranged $\mathrm{Cu} / \mathrm{Ni}$ NWs and pure $\mathrm{Cu}$ NWs were $290 \pm 20$ $\mathrm{k} \Omega, 780 \pm 40 \mathrm{k} \Omega$, and $370 \pm 30 \mathrm{k} \Omega$ respectively, See Figure 5.7. Average resistance values and uncertainties were determined by measuring the resistance values of 3 interconnect 
channels fabricated for each type of NWs arrangement. Lower resistance for well-aligned $\mathrm{Cu} / \mathrm{Ni} \mathrm{NWs}$ is attributed to the more number of effective conductive channels due to endto-end magnetic contacts as illustrated in Figure 5.8.

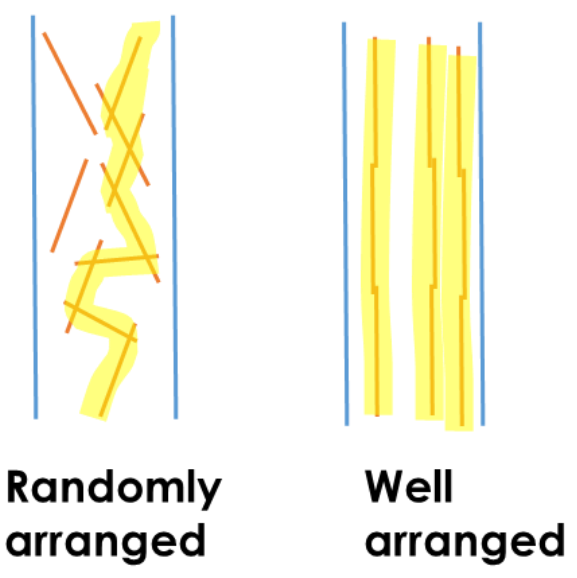

Figure 5.8. Schematic depicting the randomly aligned $\mathrm{Cu} / \mathrm{Ni}$ NWs channel fabricated in absence of magnetic field and orientationally controlled NWs channel fabricated in presence of applied magnetic field.

High resistance in case of randomly arranged $\mathrm{Cu} / \mathrm{Ni}$ NWs suggests the percolating network of NWs leads to a higher number of inter NW hops and effectively a lower number of effective channels. Whereas for the pure $\mathrm{Cu}$ NWs, the resistance is close to the wellaligned $\mathrm{Cu} / \mathrm{Ni} \mathrm{NWs}$. This could result from the high conductivity of copper in comparison to the nickel.

\subsubsection{Effect of nanowires alignment order on electrical performance}

The above studies reveal the importance of NWs alignment over randomly organized NWs on achieving a lower channel resistance. For randomly oriented nanowires, the probability of making interwire contact is uniform over the length of NW while for magnetized NWs should have a significantly higher probability near its ends ${ }^{56,131}$. This anisotropic sticking probability distribution should be a function of magnetic strength. 
Thus, the effect of applied magnetic field strength $(0-2500 \mathrm{G})$ on the alignment order and sample resistance was investigated. Optical micrographs of channels mentioned above appear in Figure 5.9. As the magnetic field increased, the resistance of NWs channels decreased, See Figure 5.10. The observation differs in comparison to the other metallic or carbon nanotube-based results ${ }^{131}$. This could be attributed to the ferromagnetic nature of nickel which enhances the end to end NW connections (Figure 5.8) increasing the number of effective channels formed.
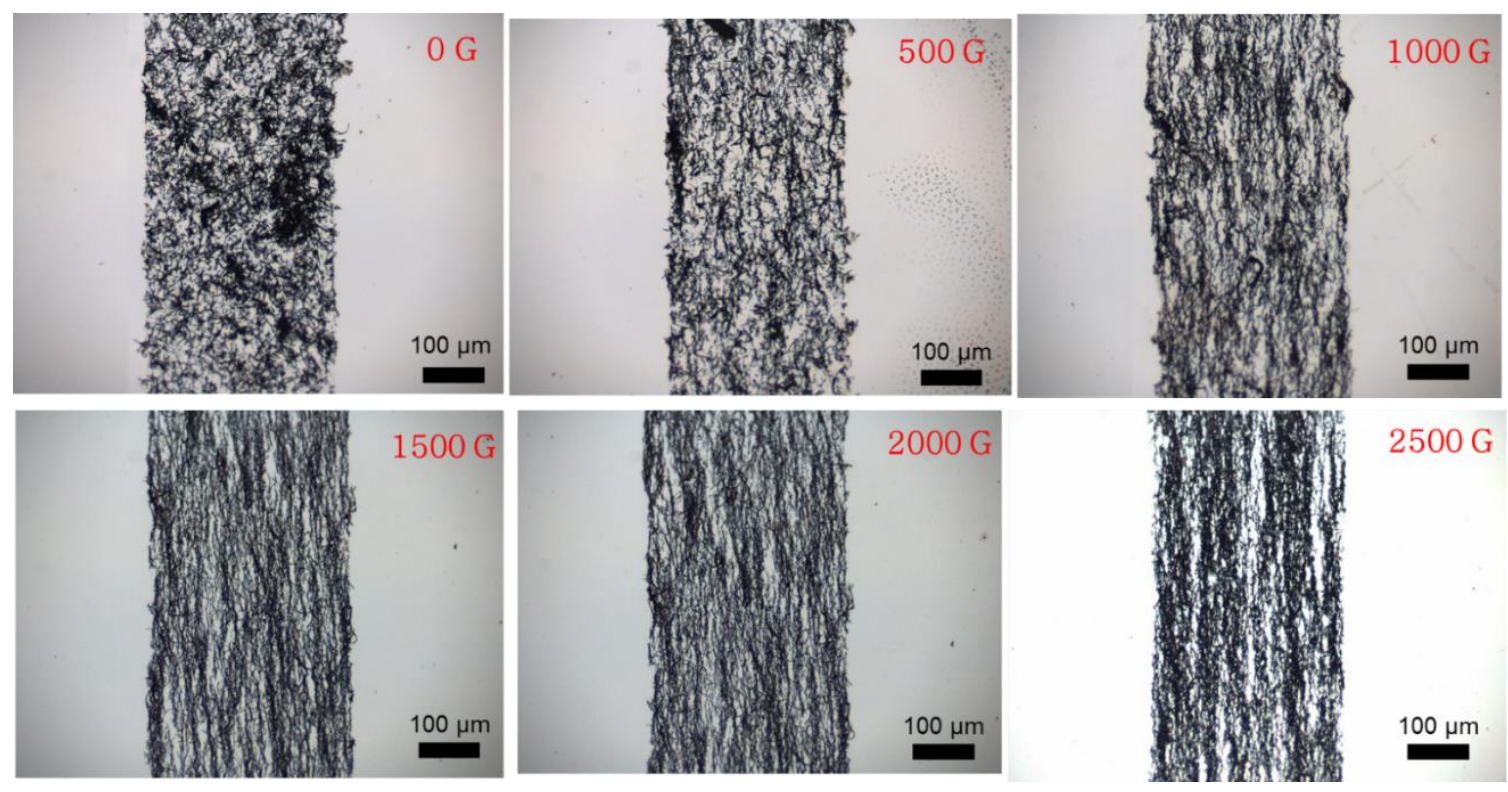

Figure 5.9. Optical microscope images of interconnect channels fabricated at various applied magnetic field strength from 0 to 2500 Gauss. 


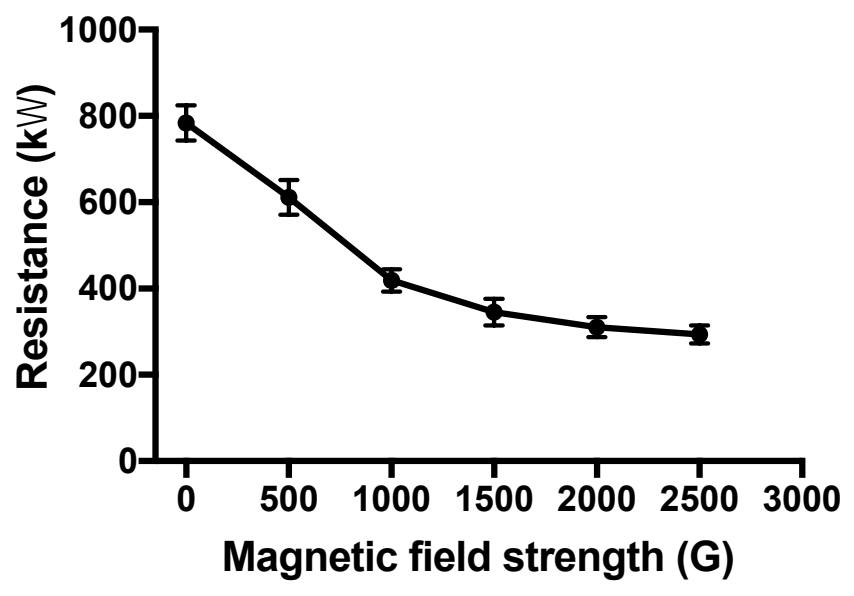

Figure 5.10. Interconnect channel resistance values of $\mathrm{Cu} / \mathrm{Ni} \mathrm{NWs}$ fabricated using various applied magnetic field strength from 0 to $2500 \mathrm{G}$.

\subsubsection{Effect of NWs density on resistance of well-aligned $\mathrm{Cu} / \mathrm{Ni}$ NWs channels}

The resistance of the NWs based interconnect channel is determined by the number of effective conductive channels that successfully allows the electrons to pass through from one end to the other end of the channel. The number of effective conductive channels can be increased by increasing the number density of NWs, i.e., increasing the NW concentration higher than required for the percolation threshold. The NW concentration in depositing suspension was increased from $0.5,1.0$, and $2.0 \mathrm{mg} / \mathrm{mL}$ for the deposition on the patterned substrate. Optical micrographs of the corresponding interconnect channels are shown in Figure 5.11. Plot in Figure 5.12, showed a decrease in the resistance values from $290 \pm 20 \mathrm{k} \Omega(0.5 \mathrm{mg} / \mathrm{mL}), 160 \pm 20 \mathrm{k} \Omega(1.0 \mathrm{mg} / \mathrm{mL})$ to $100 \pm 20 \mathrm{k} \Omega(2.0 \mathrm{mg} / \mathrm{mL})$ as the NWs density increased consistent with a simple percolation picture. 

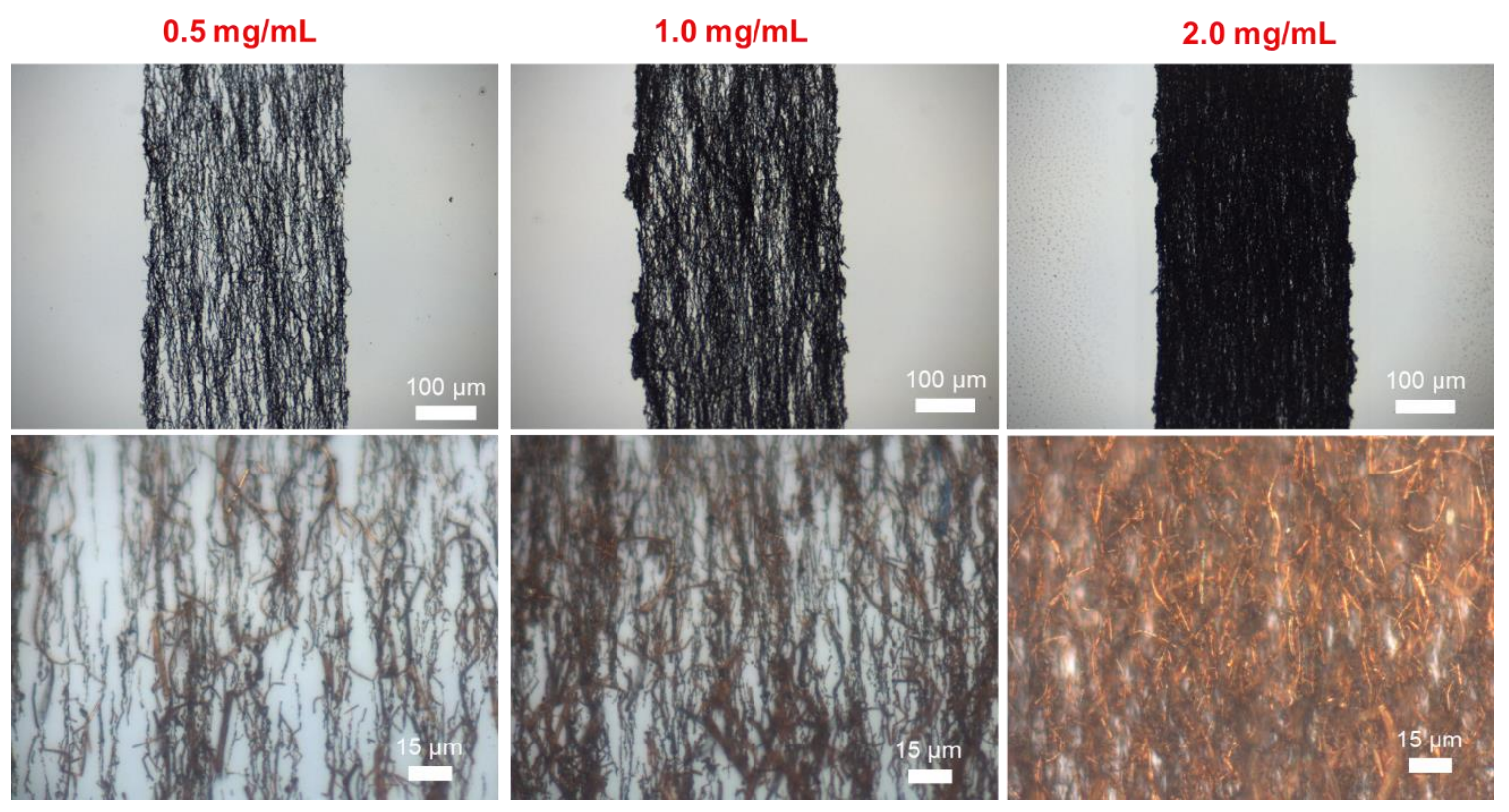

Figure 5.11. Low and high magnification optical micrographs showing of interconnect channels fabricated by using $0.5 \mathrm{mg} / \mathrm{mL}, 1.0 \mathrm{mg} / \mathrm{mL}$ and $2 \mathrm{mg} / \mathrm{mL}$ respectively.

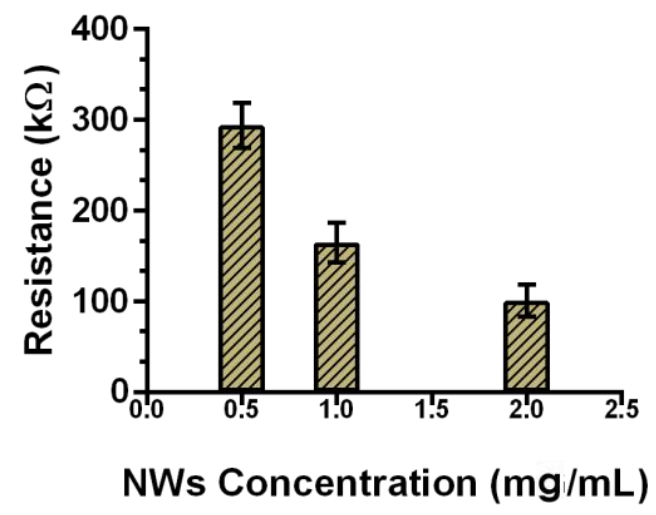

Figure 5.12. Resistance values of $\mathrm{Cu} / \mathrm{Ni}$ NWs based interconnect channels as a function of NW concentrations in suspension.

\subsubsection{Thermal stability of $\mathrm{Cu}$ NWs and $\mathrm{Cu} / \mathrm{Ni}$ NWs based interconnect channels}

The thermal stability of NWs is an important factor for the reliable performance of NWs based devices. Nickel coated NWs were found to be more oxidation resistant comparison to pure $\mathrm{Cu} \mathrm{NWs}{ }^{59}$. The thermal stability of as-fabricated interconnects channels were 
examined by heating the substrate at $100{ }^{\circ} \mathrm{C}$ in the air while simultaneously measuring the resistance. For the thermal stability studies, interconnect channels were fabricated by using $0.5 \mathrm{mg} / \mathrm{mL}$ NWs solutions. Figure 5.13 shows the resistance value as a function of heating duration for randomly arranged pure $\mathrm{Cu}$ NWs and $\mathrm{Cu} / \mathrm{Ni}$ NWs channels and well-aligned $\mathrm{Cu} / \mathrm{Ni} \mathrm{NWs}$ channels. The resistance of pure $\mathrm{Cu}$ NWs channel rapidly increased by over tenfold and crossed $20 \mathrm{M} \Omega$ in less than 10 minutes. The resistance increase resulted from the rapid oxidation of pure copper in the air. Whereas for randomly arranged and wellaligned $\mathrm{Cu} / \mathrm{Ni} \mathrm{NWs}$, the resistance rapidly increased by barely $11 \%$ and remained at constant throughout the heating duration. And even after heating for 72 hours, no significant change in resistance was observed suggesting a reliable performance of $\mathrm{Cu} / \mathrm{Ni}$ NWs at high temperature.

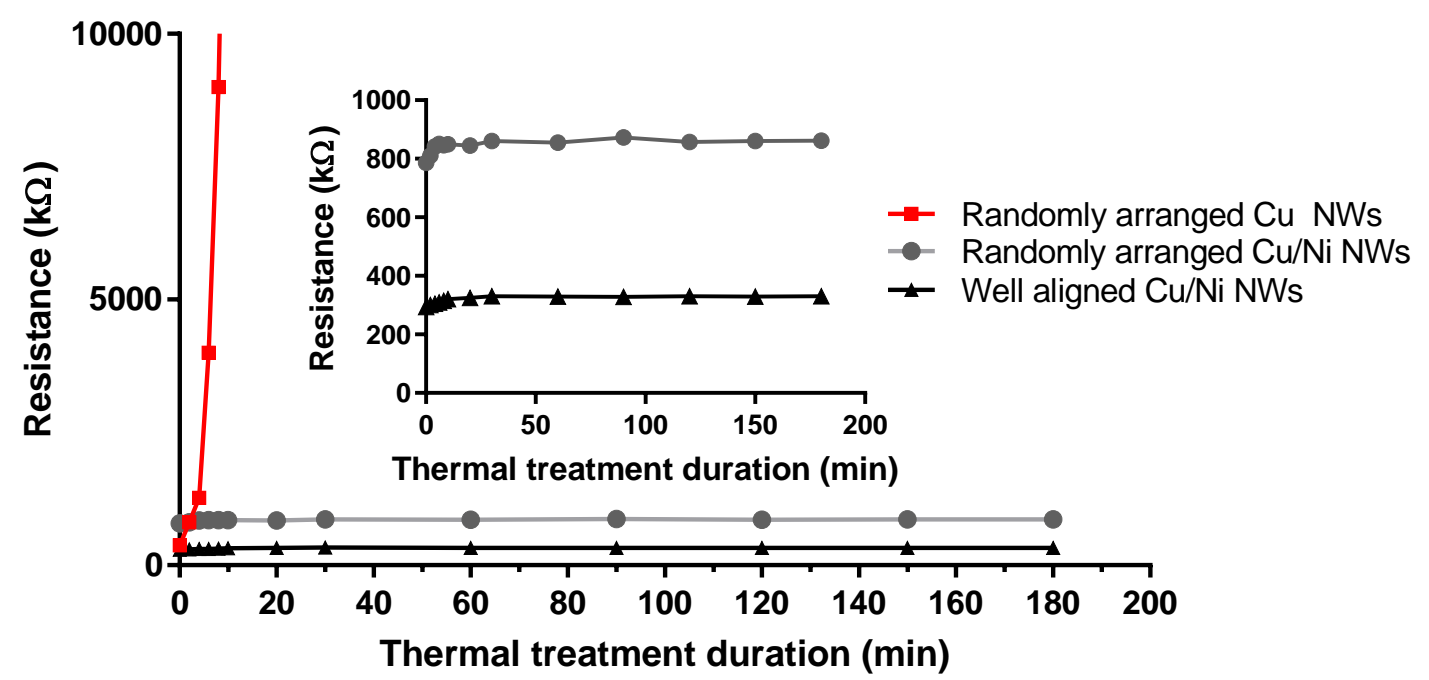

Figure 5.13. Thermal stability of well-aligned and randomly arranged $\mathrm{Cu} / \mathrm{Ni}$ NWs channels and randomly aligned $\mathrm{Cu} / \mathrm{Ni} \mathrm{NWs}$ channels at $100{ }^{\circ} \mathrm{C}$ in air. Inset plot in c) highlights the resistance curves of two type of $\mathrm{Cu} / \mathrm{Ni} \mathrm{NW}$ s arrangements. 


\subsection{Summary of $\mathrm{Cu} / \mathrm{Ni} \mathrm{NWs}$ self-assembly and electrical characterization}

Despite numerous efforts, there were no successful techniques reported for the large-scale assembly of copper nanowires. Here, these studies show the potential of the magnetic field assisted technique to control the orientation and ability to self-assemble the NWs on the large-scale area. The result was achieved by coating the copper nanowires surface with a thin layer of ferromagnetic nickel. Electrical characterization results of self-assembled $\mathrm{Cu} / \mathrm{Ni} \mathrm{NWs}$ channels suggest the importance of orientational alignment for achieving lower

resistance. Also, thermal stability studies reveal $\mathrm{Cu} / \mathrm{Ni} \mathrm{NWs}$ are highly resistant to oxidation under open-air conditions even at high temperature. 


\section{CHAPTER SIX}

\section{CONCLUSIONS AND FUTURE WORK}

\subsection{Conclusions}

As the on-chip feature sizes are getting smaller, copper filling in the ever-decreasing channels is getting difficult and reducing the process yield. Therefore, new approaches are needed to supplement the standard Dual Damascene (DD) process. Fabrication of interconnects in a bottom-up approach, using 1D nanocrystals as building blocks offers a potential solution for future high performance integrated circuits. This work demonstrates a novel and simple solution processable technique to self-assemble the $\mathrm{Cu} / \mathrm{Ni} \mathrm{NWs}$ into interconnect channels on the low- $k$ material; carbon doped silicon dioxide (CDO).

$\mathrm{CDO}$ is a highly nano-porous and hybrid low- $k$ dielectric used in ultra large-scale integration (ULSI). For the self-assembly process, CDO surface was functionalized with thiol (-SH) functional groups to bind $\mathrm{Cu} / \mathrm{Ni} \mathrm{NWs}$. Thiol functionalization was achieved via a novel approach developed to functionalize selectively the surface exposed hydrophilic Si-OH groups. Also, the same reaction was used to address the moisture uptake issue of CDO. In the chip integration process, an oxygen plasma irreversibly changes the silicon functional groups on the CDO surface from hydrophobic to hydrophilic, which causes an adverse effect on increasing the $k$ value by taking up moisture. This present work solves this issue by selectively functionalizing the surface exposed $\mathrm{Si}-\mathrm{OH}$ functional groups to hydrophobic octadecyl terminated silicon and helps in maintain the low $k$ value.

In a bottom-up method, dimensionally controlled nanowires synthesis and precisely controlling the orientation and positioning of NWs are very critical. Here, in a wet synthesis 
process, reagent concentrations and reaction temperatures were optimized to generate high aspect ratio and surface morphology controlled Cu NWs. This thesis work developed the magnetic field assisted technique to control the orientation of $\mathrm{Cu} / \mathrm{Ni}$ NWs on a large surface area. Coating a thin layer of ferromagnetic nickel (Ni) onto the $\mathrm{Cu}$ NWs surface imparted magnetic field-mediated alignment of the composite NWs. Furthermore, the nickel coating helped in protecting the copper core from oxidation.

Finally, $\mathrm{Cu} / \mathrm{Ni}$ NWs self-assembly into interconnect channels was demonstrated using the combination of photolithography and magnetic field assisted techniques. The electrical measurements revealed that well-aligned NWs exhibited less than two-fold lower resistance than the randomly organized $\mathrm{Cu} / \mathrm{Ni} \mathrm{NWs}$ channels. Also, as the degree of alignment of the NWs increased, a decrease in resistance was observed. This result is in stark contrast to the simulations results discussed for other types of NWs (Ag NWs, Au NWs and CNT). The enhanced performance of the magnetic field assisted alignment technique appeared to be related to the propensity of end-to-end contacts thereby reducing an overall number of nanowire contacts for electron transport between the two contacting electrodes. Furthermore, $\mathrm{Cu} / \mathrm{Ni}$ NWs showed a higher thermal stability than the pure $\mathrm{Cu}$ NWs, due to their high oxidation resistance. This led to a more reliable electrical performance under ambient conditions.

\subsection{Limitations}

One of the important limitation of the as developed technique is, it can only apply to the ferro/para magnetic nanowires. For non-magnetic nanowires (pure metallic or carbon nanotubes etc.), they have to be coated with a magnetically active material to give a net 
alignment when subjected to a modest magnetic field (2000 G). Here, the Cu/Ni NWs selfassembly process was demonstrated in $300 \mu \mathrm{m}$ width channels, but the future technology requires fabrication of ultra-thin $(<5 \mathrm{~nm})$ interconnect channels ${ }^{9}$. For this, the selfassembly has to be performed with ultra-thin nanowires.

Another area needing improvement is a reduction in the observed high resistance values of NWs based interconnect channels. The significant contribution to the high resistance has been attributed to NW to NW junctional resistance ${ }^{131,132}$. This nanowire junction resistance can be minimized by fusing or welding the nanowire contacts either by thermal or laser ablation techniques ${ }^{131,132}$. Also, this technique only demonstrates the NWs self-assembly in a two-dimensional manner, but it needs to be adopted for the fabrication of copper vias (vertically aligned NWs which would connect to the two adjacent layers). Therefore, more efforts are needed to apply the as developed magnetic field assisted selfassembly technique to fulfill the future interconnect fabrication needs.

\subsection{Other applications}

Copper nanowires $(\mathrm{Cu} \mathrm{NWs})$ and nickel coated $\mathrm{Cu}$ NWs $(\mathrm{Cu} / \mathrm{Ni} \mathrm{NWs})$ have been successfully demonstrated as an excellent material of choice for the preparation of highly flexible and transparent electrodes ${ }^{33,34,59,66}$. In case of Ag NWs, it was demonstrated that orientationally well-aligned NWs (deposited as a crossed arrays or mesh, like the image shown in Figure 3.11c), yields high transparency and lower sheet resistance values as opposed to randomly organized NWs arrays ${ }^{45}, 49$. Cu/Ni NWs are the better choice than Ag NWs due to its cheap precursor cost, high oxidation resistance and orientability that can be well controlled in a large-scale area. Currently, studies of $\mathrm{Cu} / \mathrm{Ni} \mathrm{NWs}$ deposition as a mesh 
on a polyethylenetetraphthalate (PET) substrate for the application of highly transparent and flexible devices are underway in our lab.

\subsection{Future work}

In this work, $300 \mu \mathrm{m}$ width and $4 \mathrm{~mm}$ lengthy channels were used to demonstrate the selfassembly process. However, one may use the magnetic field assisted technique to assemble the single NWs based ultra-thin channels. In the literature, researchers have demonstrated the synthesis of three-atom wide copper nanowires ${ }^{133}$. The developed magnetic field assisted self-assembly process is very promising, and perhaps it can be used to fabricate the three-atom wide interconnect channels which are highly desirable for future commercial chip fabrication. 


\section{REFERENCES}

1. Barceloux, D. G.; Barceloux, D., Copper. Journal of toxicology: clinical Toxicology 1999, 37 (2), 217-230.

2. Mistkawi, N. Fundamental Studies in Selective Wet Etching and Corrosion Processes for High-Performance Semiconductor Devices. Dissertation, Portland State University, Portland, 2010.

3. Mistry, K.; Allen, C.; Auth, C.; Beattie, B.; Bergstrom, D.; Bost, M.; Brazier, M.; Buehler, M.; Cappellani, A.; Chau, R. In A 45nm logic technology with high- $k+$ metal gate transistors, strained silicon, 9 Cu interconnect layers, 193nm dry patterning, and 100\% Pb-free packaging, Electron Devices Meeting, 2007. IEDM 2007. IEEE International, IEEE: 2007; pp 247-250.

4. IBM IBM's 100 Icons of Progress. http://www-

\section{3.ibm.com/ibm/history/ibm100/us/en/icons/copperchip/.}

5. Edelstein, D.; Heidenreich, J.; Goldblatt, R.; Cote, W.; Uzoh, C.; Lustig, N.;

Roper, P.; McDevitt, T.; Motsiff, W.; Simon, A. In Full copper wiring in a sub-0.25/spl mu/m CMOS ULSI technology, Electron Devices Meeting, 1997. IEDM'97. Technical Digest., International, IEEE: 1997; pp 773-776.

6. Venkatesan, S.; Gelatos, A.; Misra, V.; Smith, B.; Islam, R.; Cope, J.; Wilson, B.; Tuttle, D.; Cardwell, R.; Anderson, S., IEEE Int'l Electron Device Meeting Tech. Digest: 1997.

7. Valeev, A.; Krasnikov, G. Y., Manufacturing technology of intra-and interchip interconnects for modern ULSIs: Review and concepts of development. Russian Microelectronics 44 (3), 154-172.

8. Waveforms, P., Overview of the Use of Copper Interconnects in the Semiconductor Industry. Technology 2001, 2003, 2005.

9. Brain, R. In Interconnect scaling: Challenges and opportunities, Electron Devices Meeting (IEDM), 2016 IEEE International, IEEE: pp 9.3. 1-9.3. 4. 
10. Perry, K. A. In Chemical mechanical polishing: The impact of a new technology on an industry, VLSI Technology, 1998. Digest of Technical Papers. 1998 Symposium on, IEEE: 1998; pp 2-5.

11. Baklanov, M. R.; Adelmann, C.; Zhao, L.; De Gendt, S., Advanced Interconnects: Materials, Processing, and Reliability. ECS Journal of Solid State Science and Technology 4 (1), Y1-Y4.

12. Wetzig, K.; Wendrock, H.; Buerke, A.; Kotter, T. In In-situ study of interconnect failures by electromigration inside a scanning electron microscope, AIP Conference Proceedings, AIP: 1999; pp 89-99.

13. Cho, J.; Thompson, C., Grain size dependence of electromigration induced failures in narrow interconnects. Applied Physics Letters 1989, 54 (25), 2577-2579.

14. Bower, A.; Craft, D., Analysis of failure mechanisms in the interconnect lines of microelectronic circuits. Fatigue \&amp; fracture of engineering materials \&amp; structures 1998, 21 (5), 611-630.

15. Fischer, A.; Von Glasow, A.; Penka, S.; Ungar, F. In Electromigration failure mechanism studies on copper interconnects, Interconnect Technology Conference, 2002. Proceedings of the IEEE 2002 International, IEEE: 2002; pp 139-141.

16. Vairagar, A.; Mhaisalkar, S.; Meyer, M.; Zschech, E.; Krishnamoorthy, A.; Tu, K.; Gusak, A., Direct evidence of electromigration failure mechanism in dual-damascene $\mathrm{Cu}$ interconnect tree structures. Applied Physics Letters 2005, 87 (8), 081909.

17. Xu, W.-H.; Wang, L.; Guo, Z.; Chen, X.; Liu, J.; Huang, X.-J., Copper Nanowires as Nanoscale Interconnects: Their Stability, Electrical Transport, and Mechanical Properties. ACS nano 9 (1), 241-250.

18. Jason, N. N.; Shen, W.; Cheng, W., Copper Nanowires as Conductive Ink for Low-Cost Draw-On Electronics. ACS Applied Materials \& Interfaces 7 (30), 1676016766.

19. Kovtyukhova, N. I.; Mallouk, T. E., Nanowires as Building Blocks for Selfâ€• Assembling Logic and Memory Circuits. Chemistry-A European Journal 2002, 8 (19), 4354-4363. 
20. Xu, W.; Yu, S. H., Conducting performance of individual Ag@ C coaxial nanocables: ideal building blocks for interconnects in nanoscale devices. Small 2009, 5 (4), 460-465.

21. Mohl, M.; Pusztai, P.; Kukovecz, A.; Konya, Z.; Kukkola, J.; Kordas, K.; Vajtai, R.; Ajayan, P. M., Low-Temperature Large-Scale Synthesis and Electrical Testing of Ultralong Copper Nanowires. Langmuir 26 (21), 16496-16502.

22. Yao, Z.; Kane, C. L.; Dekker, C., High-field electrical transport in single-wall carbon nanotubes. Physical Review Letters 2000, 84 (13), 2941.

23. Gao, M. R.; Xu, W. H. H.; L.B., L.; Zhan, Y. J.; Yu, S. H., Coaxial Metal Nano/Microcables with Isolating Sheath: Synthetic Methodology and Their Application as Interconnects. Advanced Materials 22 (17), 1977-1981.

24. Wang, C.; Hu, Y.; Lieber, C. M.; Sun, S., Ultrathin Au nanowires and their transport properties. Journal of the American Chemical Society 2008, 130 (28), 89028903.

25. Critchley, K.; Khanal, B. P.; Marcin G.A. Vigderman, L.; Evans, S. D.; Zubarev, E. R.; Kotov, N. A., Near Bulk Conductivity of Gold Nanowires as Nanoscale Interconnects and the Role of Atomically Smooth Interface. Advanced Materials 22 (21), 2338-2342.

26. Molares, M. T.; Hohberger, E. M.; Schaeflein, C.; Blick, R. H.; Neumann, R.; Trautmann, C., Electrical characterization of electrochemically grown single copper nanowires. Applied Physics Letters 2003, 82 (13), 2139-2141.

27. Zach, M. P.; Ng, K. H.; Penner, R. M., Molybdenum nanowires by electrodeposition. Science 2000, 290 (5499), 2120-2123.

28. Marzi, G. D.; Iacopino, D.; Quinn, A.; Redmond, G., Probing intrinsic transport properties of single metal nanowires: direct-write contact formation using a focused ion beam. Journal of Applied Physics 2004, 96, 3458-3462.

29. Kim, T.-H.; Zhang, X.-G.; Nicholson, D. M.; Evans, B. M.; Kulkarni, N. S.; Radhakrishnan, B.; Kenik, E. A.; Li, A.-P., Large discrete resistance jump at grain boundary in copper nanowire. Nano letters 10 (8), 3096-3100. 
30. Tanase, M.; Silevitch, D.; Hultgren, A.; Bauer, L.; Searson, P.; Meyer, G.; Reich, D., Magnetic trapping and self-assembly of multicomponent nanowires. Journal of Applied Physics 2002, 91 (10), 8549-8551.

31. Mehta, R.; Chugh, S.; Chen, Z., Enhanced electrical and thermal conduction in graphene-encapsulated copper nanowires. Nano letters 15 (3), 2024-2030.

32. Li, J.; Ye, Q.; Cassell, A.; Ng, H. T.; Stevens, R.; Han, J.; Meyyappan, M., Bottom-up approach for carbon nanotube interconnects. Applied Physics Letters 2003, 82 (15), 2491-2493.

33. Ye, S.; Rathmell, A. R.; Stewart, I. E.; Ha, Y.-C.; Wilson, A. R.; Chen, Z.; Wiley, B. J., A rapid synthesis of high aspect ratio copper nanowires for high-performance transparent conducting films. Chemical Communications 50 (20), 2562-2564.

34. Rathmell, A. R.; Wiley, B. J., The synthesis and coating of long, thin copper nanowires to make flexible, transparent conducting films on plastic substrates. Advanced Materials 23 (41), 4798-4803.

35. Ye, S.; Stewart, I. E.; Chen, Z.; Li, B.; Rathmell, A. R.; Wiley, B. J., How Copper Nanowires Grow and How To Control Their Properties. Accounts of chemical research 49 (3), 442-451.

36. Raciti, D.; Livi, K. J.; Wang, C., Highly dense Cu nanowires for lowoverpotential CO2 reduction. Nano Letters 15 (10), 6829-6835.

37. Ryan, J. G.; Geffken, R. M.; Poulin, N. R.; Paraszczak, J. R., The evolution of interconnection technology at IBM. Ibm Journal of Research and Development 1995, 39 (4), 371-381.

38. Wang, M. C. P.; Gates, B. D., Directed assembly of nanowires. Materials Today 2009, 12 (5), 34-43.

39. Tamhane, K. Formation of lyotropic liquid crystals through the self-assembly of bile acid building blocks. University of Central Florida Orlando, Florida, 2009. 40. Murali, S.; Xu, T.; Marshall, B. D.; Kayatin, M. J.; Pizarro, K.; Radhakrishnan, V. K.; Nepal, D.; Davis, V. A., Lyotropic liquid crystalline self-assembly in dispersions of silver nanowires and nanoparticles. Langmuir 26 (13), 11176-11183. 
41. Liu, J.-W.; Liang, H.-W.; Yu, S.-H., Macroscopic-Scale Assembled Nanowire Thin Films and Their Functionalities. Chemical Reviews 112 (8), 4770-4799.

42. Lu, W.; Lieber, C. M., Nanoelectronics from the bottom up. Nature materials 2007, 6 (11), 841-850.

43. Lieber, C. M., Nanoscale science and technology: building a big future from small things. MRS bulletin 2003, 28 (07), 486-491.

44. Tao, A.; Kim, F.; Hess, C.; Goldberger, J.; He, R.; Sun, Y.; Xia, Y.; Yang, P., Langmuir-Blodgett silver nanowire monolayers for molecular sensing using surfaceenhanced Raman spectroscopy. Nano Letters 2003, 3 (9), 1229-1233.

45. Cho, S.; Kang, S.; Pandya, A.; Shanker, R.; Khan, Z.; Lee, Y.; Park, J.; Craig, S. L.; Ko, H., Large-Area Cross-Aligned Silver Nanowire Electrodes for Flexible, Transparent, and Force-Sensitive Mechanochromic Touch Screens. ACS nano 11 (4), 4346-4357.

46. Li, D.; Wang, Y.; Xia, Y., Electrospinning of Polymeric and Ceramic Nanofibers as Uniaxially Aligned Arrays. Nano Letters 2003, 3 (8), 1167-1171.

47. Smith, P. A.; Nordquist, C. D.; Jackson, T. N.; Mayer, T. S.; Martin, B. R.; Mbindyo, J.; Mallouk, T. E., Electric-field assisted assembly and alignment of metallic nanowires. Applied Physics Letters 2000, 77 (9), 1399-1401.

48. Kim, Y. L.; Li, B.; An, X.; Hahm, M. G.; Chen, L.; Washington, M.; Ajayan, P.; Nayak, S. K.; Busnaina, A.; Kar, S., Highly aligned scalable platinum-decorated singlewall carbon nanotube arrays for nanoscale electrical interconnects. ACS nano 2009, 3 (9), 2818-2826.

49. Yang, Y.; Wang, J.-L.; Liu, L.; Wang, Z.-H.; Liu, J.-W.; Yu, S.-H., A roomtemperature environmentally friendly solution process to assemble silver nanowire architectures for flexible transparent electrodes. Nanoscale 9 (1), 52-55.

50. Ye, H.; Gu, Z.; Yu, T.; Gracias, D. H., Integrating nanowires with substrates using directed assembly and nanoscale soldering. IEEE Transactions on nanotechnology 2006, 5 (1), 62-66.

51. Hangarter, C. M.; Myung, N. V., Magnetic alignment of nanowires. Chemistry of materials 2005, 17 (6), 1320-1324. 
52. Fan, Z.; Ho, J. C.; Jacobson, Z. A.; Yerushalmi, R.; Alley, R. L.; Razavi, H.; Javey, A., Wafer-scale assembly of highly ordered semiconductor nanowire arrays by contact printing. Nano Letters 2008, 8 (1), 20-25.

53. Choi, H.; Park, S.-H., Seedless growth of free-standing copper nanowires by chemical vapor deposition. Journal of the American Chemical Society 2004, 126 (20), 6248-6249.

54. Barako, M. T.; Roy-Panzer, S.; English, T. S.; Kodama, T.; Asheghi, M.; Kenny, T. W.; Goodson, K. E., Thermal conduction in vertically aligned copper nanowire arrays and composites. ACS applied materials \& interfaces 7 (34), 19251-19259.

55. Zhou, R.; Chang, H.-C.; Protasenko, V.; Kuno, M.; Singh, A. K.; Jena, D.; Xing, H., CdSe nanowires with illumination-enhanced conductivity: Induced dipoles, dielectrophoretic assembly, and field-sensitive emission. Journal of Applied Physics 2007, 101 (7), 073704.

56. Yoo, B.; Rheem, Y.; Beyermann, W. P.; Myung, N. V., Magnetically assembled $30 \mathrm{~nm}$ diameter nickel nanowire with ferromagnetic electrodes. Nanotechnology 2006, 17 (10), 2512.

57. Chen, H.; Lee, J.-H.; Kim, Y.-H.; Shin, D.-W.; Park, S.-C.; Meng, X.; Yoo, J.-B., Metallic copper nanostructures synthesized by a facile hydrothermal method. Journal of nanoscience and nanotechnology 10 (1), 629-636.

58. Chang, Y.; Lye, M. L.; Zeng, H. C., Large-scale synthesis of high-quality ultralong copper nanowires. Langmuir 2005, 21 (9), 3746-3748.

59. Rathmell, A. R.; Nguyen, M.; Chi, M.; Wiley, B. J., Synthesis of oxidationresistant cupronickel nanowires for transparent conducting nanowire networks. Nano Letters 12 (6), 3193-3199.

60. Darmakkolla, S. R.; Tran, H.; Gupta, A.; Rananavare, S. B., A method to derivatize surface silanol groups to Si-alkyl groups in carbon-doped silicon oxides. $R S C$ Advances 6 (95), 93219-93230.

61. Andideh, E.; Peterson, K. L., Method of forming a carbon doped oxide layer on a substrate. Google Patents: 2002. 
62. Chang, T. C.; Mor, Y. S.; Liu, P. T.; Tsai, T. M.; Chen, C. W.; Mei, Y. J.; Sze, S. M., Recovering dielectric loss of low dielectric constant organic siloxane during the photoresist removal process. Journal of the Electrochemical Society 2002, 149 (8), F81F84.

63. Humphreys, F.; Brough, I., High resolution electron backscatter diffraction with a field emission gun scanning electron microscope. Journal of microscopy 1999, 195 (Pt 1), 6-9.

64. Trimby, P. W., Orientation mapping of nanostructured materials using transmission Kikuchi diffraction in the scanning electron microscope. Ultramicroscopy $120,16-24$.

65. Keller, R. R.; Geiss, R. H., Transmission EBSD from $10 \mathrm{~nm}$ domains in a scanning electron microscope. Journal of microscopy 245 (3), 245-251.

66. Rathmell, A. R.; Bergin, S. M.; Hua, Y. L.; Li, Z. Y.; Wiley, B. J., The growth mechanism of copper nanowires and their properties in flexible, transparent conducting films. Advanced Materials 22 (32), 3558-3563.

67. Tung, H.-T.; Song, J.-M.; Dong, T.-Y.; Hwang, W.-S.; Chen, I.-G., Synthesis of Surfactant-free Aligned Single Crystal Copper Nanowires by Thermal-Assisted Photoreduction. Crystal Growth \& Design 2008, 8 (9), 3415-3419.

68. Meng, F.; Jin, S., The solution growth of copper nanowires and nanotubes is driven by screw dislocations. Nano letters 12 (1), 234-239.

69. Kim, M. J.; Flowers, P. F.; Stewart, I. E.; Ye, S.; Baek, S.; Kim, J. J.; Wiley, B. J., Ethylenediamine Promotes $\mathrm{Cu}$ Nanowire Growth by Inhibiting Oxidation of $\mathrm{Cu}$ (111). Journal of the American Chemical Society 139 (1), 277-284.

70. Niu, Z.; Li, Y., Removal and utilization of capping agents in nanocatalysis. Chemistry of materials 26 (1), 72-83.

71. Ye, S.; Rathmell, A. R.; Ha, Y. C.; Wilson, A. R.; Wiley, B. J., The Role of Cuprous Oxide Seeds in the One Pot and Seeded Syntheses of Copper Nanowires. Small $10(9), 1771-1778$. 
72. Stewart, I. E.; Ye, S.; Chen, Z.; Flowers, P. F.; Wiley, B. J., Synthesis of Cu-Ag, $\mathrm{Cu}-\mathrm{Au}$, and $\mathrm{Cu}-\mathrm{Pt}$ core-shell nanowires and their use in transparent conducting films. Chemistry of Materials 27 (22), 7788-7794.

73. Li, J.; Shacham-Diamand, Y.; Mayer, J. W., Copper deposition and thermal stability issues in copper-based metallization for ULSI technology. Materials science reports 1992, 9 (1), 1-51.

74. Murarka, S. P.; Hymes, S. W., Copper metallization for ULSL and beyond. Critical Reviews in Solid State and Material Sciences 1995, 20 (2), 87-124.

75. Venkatesan, S.; Gelatos, A.; Hisra, S.; Smith, B.; Islam, R.; Cope, J.; Wilson, B.; Tuttle, D.; Cardwell, R.; Anderson, S. In A high performance $1.8 \mathrm{~V}, 0.20 / \mathrm{spl} \mathrm{mu} / \mathrm{m}$ CMOS technology with copper metallization, Electron Devices Meeting, 1997. IEDM'97. Technical Digest., International, IEEE: 1997; pp 769-772.

76. Jing, S.-Y.; Lee, H.-J.; Choi, C. K., Chemical bond structure on Si-OC composite films with a low dielectric constant deposited by using inductively coupled plasma chemical vapor deposition. Journal of the Korean Physical Society 2002, 41 (5), 769-773. 77. Kim, J. H.; Seo, S. H.; Yun, S. M.; Chang, H. Y.; Lee, K. M.; Choi, C. K., The deposition of SiOF film with low dielectric constant in a helicon plasma source. Applied Physics Letters 1996, 68 (11), 1507-1509.

78. Kim, J. H.; Seo, S. H.; Yun, S. M.; Chang, H. Y.; Lee, K. M.; Choi, C. K., A study on low dielectric material deposition using a helicon plasma source. Journal of the Electrochemical Society 1996, 143 (9), 2990-2995.

79. Grill, A.; Patel, V., Ultralow-k dielectrics prepared by plasma-enhanced chemical vapor deposition. Applied Physics Letters 2001, 79 (6), 803-805.

80. Grill, A.; Patel, V., Low dielectric constant films prepared by plasma-enhanced chemical vapor deposition from tetramethylsilane. Journal of Applied Physics 1999, 85 (6), 3314-3318.

81. Fujii, T.; Hiramatsu, M.; Nawata, M., Formation of Si-based organic thin films with low dielectric constant by using remote plasma enhanced chemical vapor deposition from hexamethyldisiloxane. Thin Solid Films 1999, 343, 457-460. 
82. Grill, A.; Neumayer, D. A., Structure of low dielectric constant to extreme low dielectric constant SiCOH films: Fourier Transform infrared spectroscopy characterization. Journal of Applied Physics 2003, 94 (10), 6697-6707.

83. Yang, C. S.; Yu, Y. H.; Lee, K.-M.; Lee, H.-J.; Choi, C. K., Investigation of low dielectric carbon-doped silicon oxide films prepared by PECVD using methyltrimethoxysilane precursor. Thin Solid Films 2006, 506, 50-54.

84. Han, Q.; Chen, W.; Waldfried, C.; Escorcia, O.; Sbrockey, N.; Bridgewater, T.; Moyer, E.; Berry, I. In Ultra low-k porous silicon dioxide films from a plasma process, Interconnect Technology Conference, 2001. Proceedings of the IEEE 2001 International, IEEE: 2001; pp 171-173.

85. Wang, Y. H.; Kumar, R.; Zhou, X.; Pan, J. S.; Chai, J. W., Effect of oxygen plasma treatment on low dielectric constant carbon-doped silicon oxide thin films. Thin Solid Films 2005, 473 (1), 132-136.

86. Reichelderfer, R. F.; Welty, J. M.; Battey, J. F., The Ultimate By-Products of Stripping Photoresist in an Oxygen Plasma. Journal of the Electrochemical Society 1977, 124 (12), 1926-1927.

87. Chang, T. C.; Chen, C. W.; Liu, P. T.; Mor, Y. S.; Tsai, H. M.; Tsai, T. M.; Yan, S. T.; Tu, C. H.; Tseng, T. Y.; Sze, S. M., Moisture-induced material instability of porous organosilicate glass. Electrochemical and Solid State Letters 2003, 6 (4), F13-F15.

88. Liu, P. T.; Chang, T. C.; Su, H.; Mor, Y. S.; Yang, Y. L.; Chung, H.; Hou, J.; Sze, S. M., Improvement in integration issues for organic low-k hybrid-organic-siloxanepolymer. Journal of the Electrochemical Society 2001, 148 (2), F30-F34.

89. Wang, Z.; Catabay, W. G.; Zhao, J. W., Process for treating damaged surfaces of low dielectric constant organo silicon oxide insulation material to inhibit moisture absorption. Google Patents: 2000.

90. Le, Q. T.; Baklanov, M.; Kesters, E.; Azioune, A.; Struyf, H.; Boullart, W.; Pireaux, J.-J.; Vanhaelemeersch, S., Removal of plasma-modified low-k layer using dilute HF: Influence of concentration. Electrochemical and Solid-State Letters 2005, 8 (7), F21-F24. 
91. An, D.; Wang, Z.; Zhao, X.; Liu, Y.; Guo, Y.; Ren, S., A new route to synthesis of surface hydrophobic silica with long-chain alcohols in water phase. Colloids and Surfaces a-Physicochemical and Engineering Aspects 2010, 369 (1-3), 218-222.

92. Rao, C. N. R.; Muller, A.; Cheetham, A. K., The chemistry of nanomaterials: synthesis, properties and applications. John Wiley \&amp; Sons: 2006; Vol. 1.

93. Gurav, J. L.; Jung, I.-K.; Park, H.-H.; Kang, E. S.; Nadargi, D. Y., Silica aerogel: synthesis and applications. Journal of Nanomaterials 2010, 23.

94. Dorcheh, A. S.; Abbasi, M. H., Silica aerogel; synthesis, properties and characterization. Journal of Materials Processing Technology 2008, 199 (1-3), 10-26. 95. Gurav, J. L.; Jung, I.-K.; Park, H.-H.; Kang, E. S.; Nadargi, D. Y., Silica Aerogel: Synthesis and Applications. Journal of Nanomaterials 2010.

96. Ashu-Arrah, B. A.; Glennon, J. D.; Albert, K., Preparation and characterization of bonded silica hydride intermediate from triethoxysilane and dimethylmethoxysilane using supercritical carbon dioxide and dioxane as reaction medium. Journal of

Chromatography A 2012, 1236, 42-50.

97. Jal, P. K.; Patel, S.; Mishra, B., Chemical modification of silica surface by immobilization of functional groups for extractive concentration of metal ions. Talanta 2004, 62 (5), 1005-1028.

98. Kartal, A. M.; Erkey, C., Surface modification of silica aerogels by hexamethyldisilazane-carbon dioxide mixtures and their phase behavior. The Journal of Supercritical Fluids 2010, 53 (1), 115-120.

99. Suratwala, T. I.; Hanna, M. L.; Miller, E. L.; Whitman, P. K.; Thomas, I. M.; Ehrmann, P. R.; Maxwell, R. S.; Burnham, A. K., Surface chemistry and trimethylsilyl functionalization of Stober silica sols. Journal of Non-Crystalline Solids 2003, 316 (2-3), 349-363.

100. Zareyee, D.; Karimi, B., A novel and highly efficient method for the silylation of alcohols with hexamethyldisilazane (HMDS) catalyzed by recyclable sulfonic acidfunctionalized ordered nanoporous silica. Tetrahedron Letters 2007, 48 (7), 1277-1280. 
101. Nadargi, D. Y.; Gurav, J. L.; El Hawi, N.; Rao, A. V.; Koebel, M., Synthesis and characterization of transparent hydrophobic silica thin films by single step sol-gel process and dip coating. Journal of Alloys and Compounds 2010, 496 (1-2), 436-441.

102. Shewale, P. M.; Rao, A. V.; Rao, A. P., Effect of different trimethyl silylating agents on the hydrophobic and physical properties of silica aerogels. Applied Surface Science 2008, 254 (21), 6902-6907.

103. Rao, A. V.; Kulkarni, M. M.; Amalnerkar, D. P.; Seth, T., Surface chemical modification of silica aerogels using various alkyl-alkoxy/chloro silanes. Applied Surface Science 2003, 206 (1-4), 262-270.

104. Mhaisagar, Y. S.; Joshi, B. N.; Mahajan, A. M., Mechanical properties of surface modified silica low-k thin films. Microelectronic Engineering 2014, 114, 112-116.

105. Zhuravlev, L. T., Concentration of hydroxyl-groups on the surface of amorphous silicas. Langmuir 1987, 3 (3), 316-318.

106. Sandoval, J. E.; Pesek, J. J., Synthesis and characterization of a hydride -modified porous silica material as an intermediate in the preparation of chemically bonded chromatographic stationary phases. Analytical Chemistry 1989, 61 (18), 2067-2075. 107. Voronkov, M. G.; Pukhnarevich, V. B., Properties and transformations of the Si$\mathrm{H}$ bonds in organosilicon compounds. Bulletin of the Academy of Sciences of the USSR, Division of chemical science 1982, 31 (5), 939-957.

108. Buriak, J. M., Organometallic chemistry on silicon and germanium surfaces. Chemical Reviews 2002, 102 (5), 1271-1308.

109. Wayner, D. D. M.; Wolkow, R. A., Organic modification of hydrogen terminated silicon surfaces. Journal of the Chemical Society-Perkin Transactions 2 2002, (1), 23-34. 110. Tour, J. M.; John, J. A.; Stephens, E. B., Convenient route to diorganosilyl and triorganosilyl ethy ethers and the corresponding diorganosilanes and triorganosilanes. Journal of Organometallic Chemistry 1992, 429 (3), 301-310.

111. Anderson Jr, J. H.; Parks, G. A., Electrical conductivity of silica gel in the presence of adsorbed water. The Journal of Physical Chemistry 1968, 72 (10), 36623668. 
112. Sakamoto, T.; Nakamura, H.; Uedaira, H.; Wada, A., High-frequency dielectric relaxation of water bound to hydrophilic silica gels. The Journal of Physical Chemistry 1989, 93 (1), 357-366.

113. Knight, R. J.; Nur, A., The dielectric constant of sandstones, $60 \mathrm{kHz}$ to $4 \mathrm{MHz}$. Geophysics 1987, 52 (5), 644-654.

114. Friedman, S. P., A saturation degree-dependent composite spheres model for describing the effective dielectric constant of unsaturated porous media. Water Resources Research 1998, 34 (11), 2949-2961.

115. Afsar, M. N.; Button, K. J., Precise Millimeter-Wave Measurements of Complex Refractive Index, Complex Dielectric Permittivity and Loss Tangent of GaAs, Si, $\mathrm{SiO} / \mathrm{sub} 2 /, \mathrm{A} 1 / \mathrm{sub} 2 / \mathrm{O} / \mathrm{sub} 3 /, \mathrm{BeO}$, Macor, and Glass. IEEE Transactions on Microwave Theory and Techniques 1983, 31 (2), 217-223.

116. Reis, J. C. R.; Lampreia, I. M. S.; Santos, Â. F. S.; Moita, M. L. C. J.; Douhéret, G., Refractive Index of Liquid Mixtures: Theory and Experiment. ChemPhysChem 11 (17), 3722-3733.

117. de Smet, L. C.; Zuilhof, H.; Sudholter, E. J.; Lie, L. H.; Houlton, A.; Horrocks, B. R., Mechanism of the hydrosilylation reaction of alkenes at porous silicon: experimental and computational deuterium labeling studies. The Journal of Physical Chemistry B 2005, 109 (24), 12020-12031.

118. Cassie, A., Contact angles. Discussions of the Faraday Society 1948, 3, 11-16. 119. Koga, T.; Morita, M.; Ishida, H.; Yakabe, H.; Sasaki, S.; Sakata, O.; Otsuka, H.; Takahara, A., Dependence of the molecular aggregation state of octadecylsiloxane monolayers on preparation methods. Langmuir 2005, 21 (3), 905-910.

120. Love, J. C.; Estroff, L. A.; Kriebel, J. K.; Nuzzo, R. G.; Whitesides, G. M., Selfassembled monolayers of thiolates on metals as a form of nanotechnology. Chemical Reviews 2005, 105 (4), 1103-1170.

121. Jennings, G. K.; Munro, J. C.; Yong, T.-H.; Laibinis, P. E., Effect of chain length on the protection of copper by n-alkanethiols. Langmuir 1998, 14 (21), 6130-6139. 
122. Jennings, G. K.; Laibinis, P. E., Self-assembled monolayers of alkanethiols on copper provide corrosion resistance in aqueous environments. Colloids and Surfaces A: Physicochemical and Engineering Aspects 1996, 116 (1-2), 105-114.

123. Gelves, G. A.; Sundararaj, U.; Haber, J. A., Electrostatically dissipative polystyrene nanocomposites containing copper nanowires. Macromolecular rapid communications 2005, 26 (21), 1677-1681.

124. Chaparadza, A.; Rananavare, S. B., Room temperature $\mathrm{Cl} 2$ sensing using thick nanoporous films of Sb-doped SnO 2. Nanotechnology 2008, 19 (24), 245501.

125. Chan, J. C.; Hannah-Moore, N.; Rananavare, S. B., Controlled Deposition of Tin Oxide and Silver Nanoparticles Using Microcontact Printing. Crystals 2015, 5 (1), 116142.

126. Jonkheijm, P.; Weinrich, D.; Schroder, H.; Niemeyer, C. M.; Waldmann, H., Chemical strategies for generating protein biochips. Angewandte Chemie International Edition 2008, 47 (50), 9618-9647.

127. Tallapally, V.; Esteves, R. J. A.; Nahar, L.; Arachchige, I. U., Multivariate Synthesis of Tin Phosphide Nanoparticles: Temperature, Time, and Ligand Control of Size, Shape, and Crystal Structure. Chemistry of Materials 28 (15), 5406-5414.

128. Grill, A., Amorphous carbon based materials as the interconnect dielectric in ULSI chips. Diamond and related materials 2001, 10 (2), 234-239.

129. Morakinyo, M. K.; Rananavare, S. B., Reducing the effects of shot noise using nanoparticles. Journal of Materials Chemistry C 3 (5), 955-959.

130. Hu, M.; Noda, S.; Okubo, T.; Yamaguchi, Y.; Komiyama, H., Structure and morphology of self-assembled 3-mercaptopropyltrimethoxysilane layers on silicon oxide. Applied Surface Science 2001, 181 (3), 307-316.

131. Mutiso, R. M.; Sherrott, M. C.; Rathmell, A. R.; Wiley, B. J.; Winey, K. I., Integrating simulations and experiments to predict sheet resistance and optical transmittance in nanowire films for transparent conductors. ACS nano 7 (9), 7654-7663. 132. Garnett, E. C.; Cai, W.; Cha, J. J.; Mahmood, F.; Connor, S. T.; Christoforo, M. G.; Cui, Y.; McGehee, M. D.; Brongersma, M. L., Self-limited plasmonic welding of silver nanowire junctions. Nature materials 11 (3), 241-249. 
133. Yan, H.; Hohman, J. N.; Li, F. H.; Jia, C.; Solis-Ibarra, D.; Wu, B.; Dahl, J. E. P.; Carlson, R. M. K.; Tkachenko, B. A.; Fokin, A. A.; Schreiner, P. R.; Vailionis, A.; Kim, T. R.; Devereaux, T. P.; Shen, Z.-X.; Melosh, N. A., Hybrid metal-organic chalcogenide nanowires with electrically conductive inorganic core through diamondoid-directed assembly. Nat Mater 16 (3), 349-355. 


\section{APPENDICES}

\section{Appendix-A. Measurement of nanowire length distribution}

The average size of nanowires and its distribution were measured by uploading SEM or optical micrographs into the Image-J application. The analysis employed the distance measuring tool as shown in Figure A. The typical sample size consisted of well over 250 nanowires from each batch.

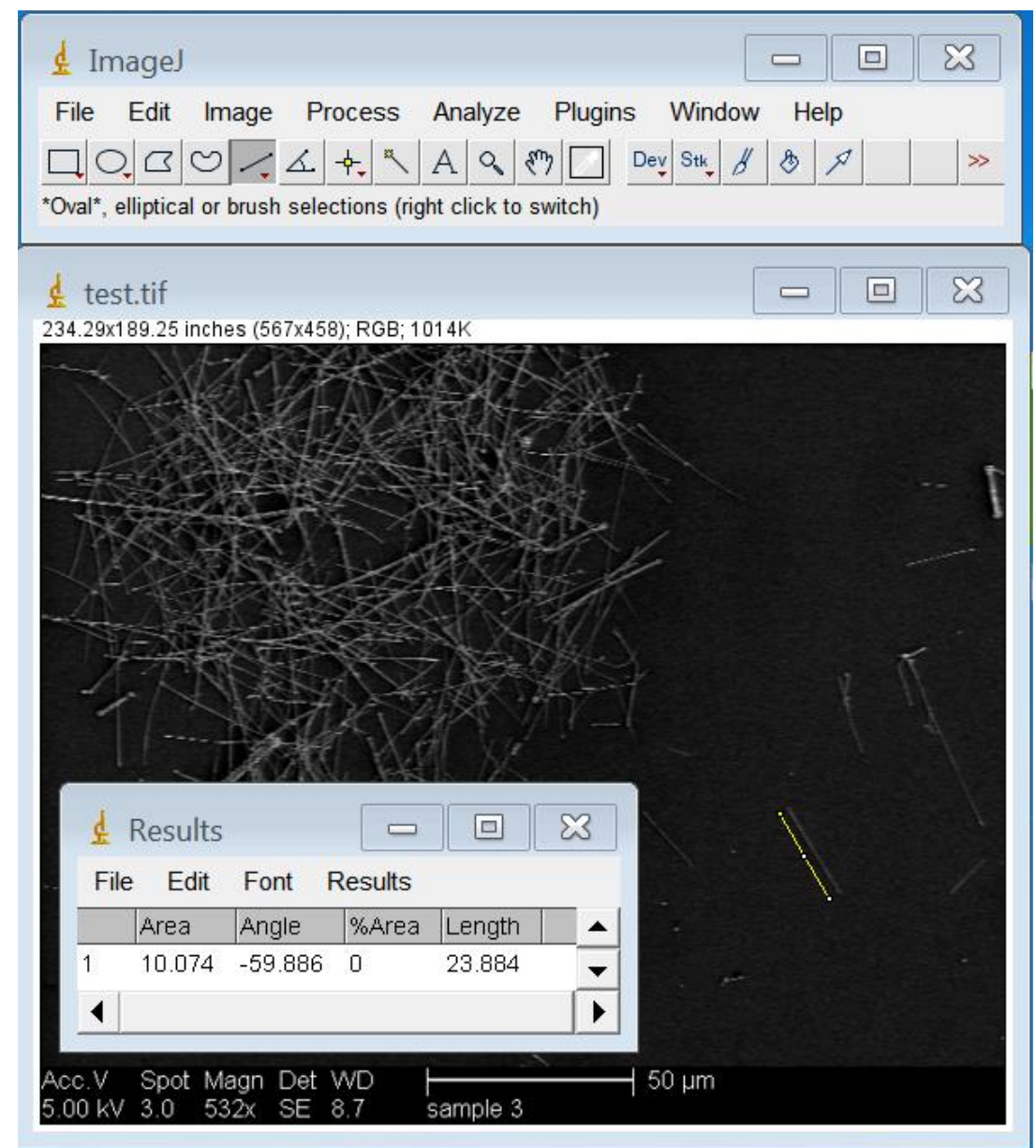

Figure A. SEM image and Image-J application depicting the nanowire length measurement technique. 


\section{Appendix-B. Measurement of nanowires alignment angle}
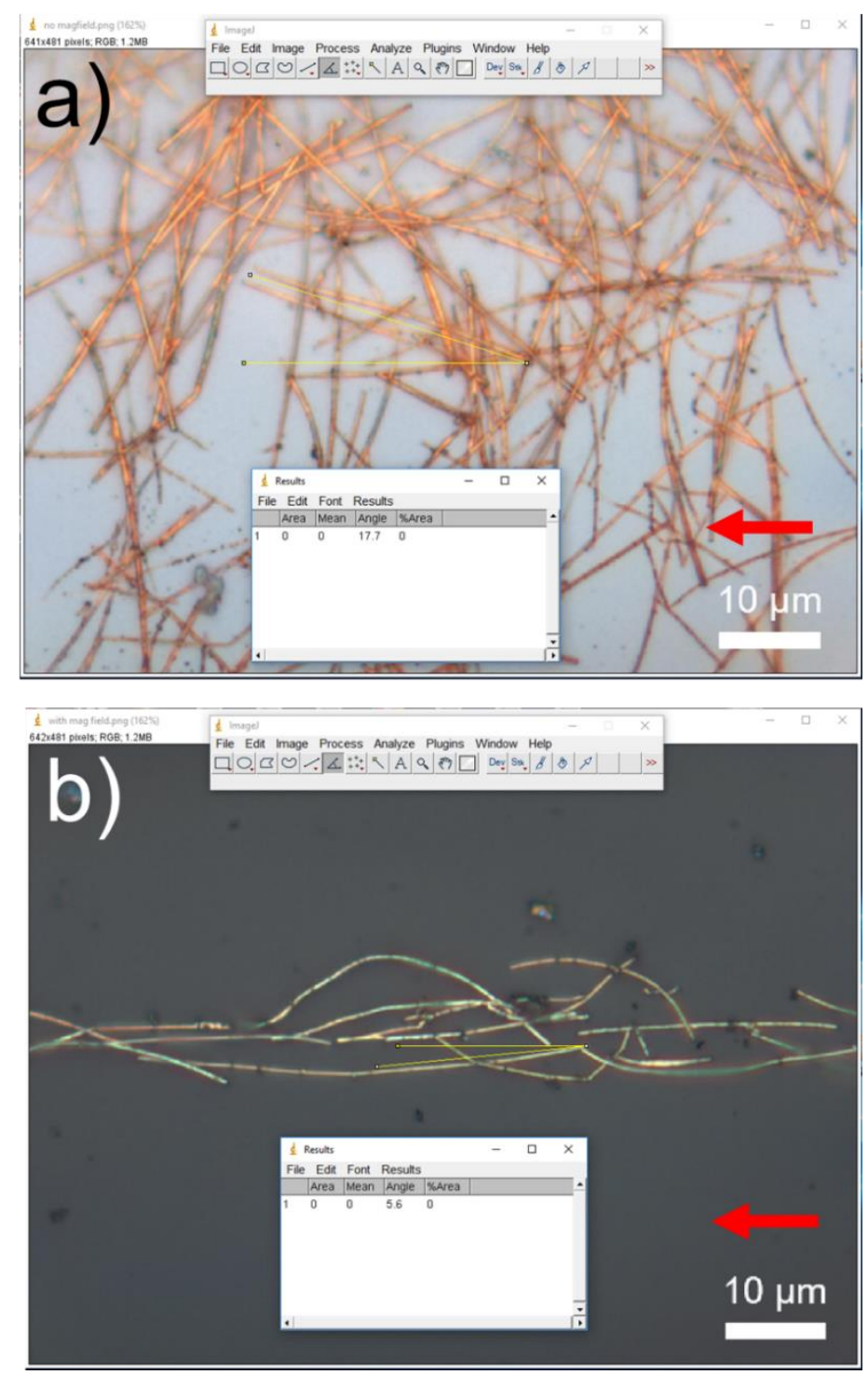

Figure B. Optical micrographs depicting the nanowire alignment angle measurement using Image$\mathrm{J}$ application for NWs dried in the absence (a), and in the presence of applied magnetic field (b). The red arrow in image-a represents the hypothesized axis and in image-b represents the applied magnetic field direction.

The $\mathrm{Cu} / \mathrm{Ni}$ nanowires alignment angle distribution was determined by using Image-

$\mathrm{J}$ application. The procedure involved uploading the optical micrographs into the Image-J 
application. The measurement angular distribution was performed with respect to a randomly chosen orientation (in the absence of magnetic field) or applied magnetic field direction. These studies utilized images such as shown in Figure B. Several (>250 NWs) isolated nanowires, with two visible ends, were analyzed for NWs alignment angle distribution and average alignment angles.

\section{Appendix-C. Issues of using ultra-long ( $>30 \mu \mathrm{m})$ nanowires}

As the length of the $\mathrm{Cu} / \mathrm{Ni}$ nanowires increases, the flexibility also increases. Especially, the magnetic interactions enhance the bending nature of nanowires resulting in permanently bent nanowire arrangements, see Figure C. The interconnect fabrication requires the nanowires to be deposited as the straight conductive channels. Therefore, for the current study, nanowires with the length of less than $30 \mu \mathrm{m}$ were used.

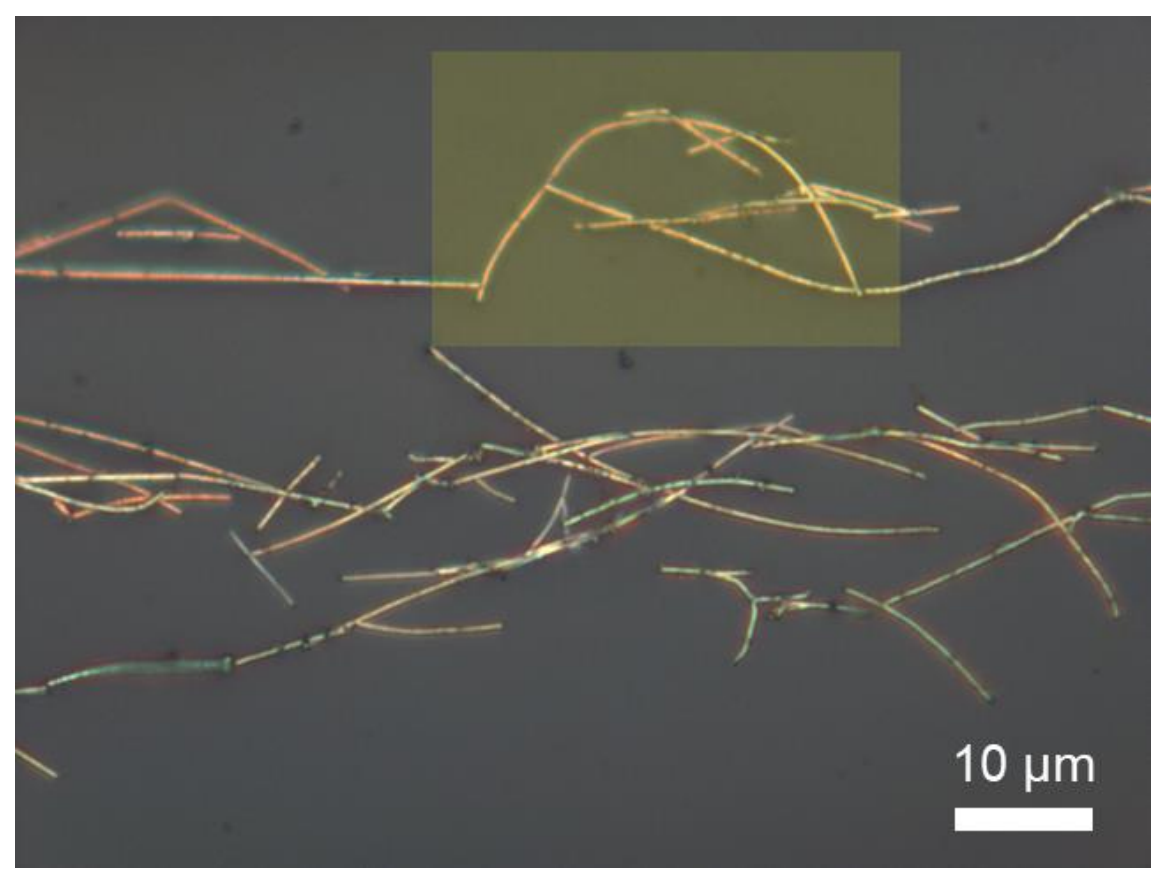

Figure C. Optical micrograph highlighting the flexibility of ultra-long nanowire (shown in the yellow box). 